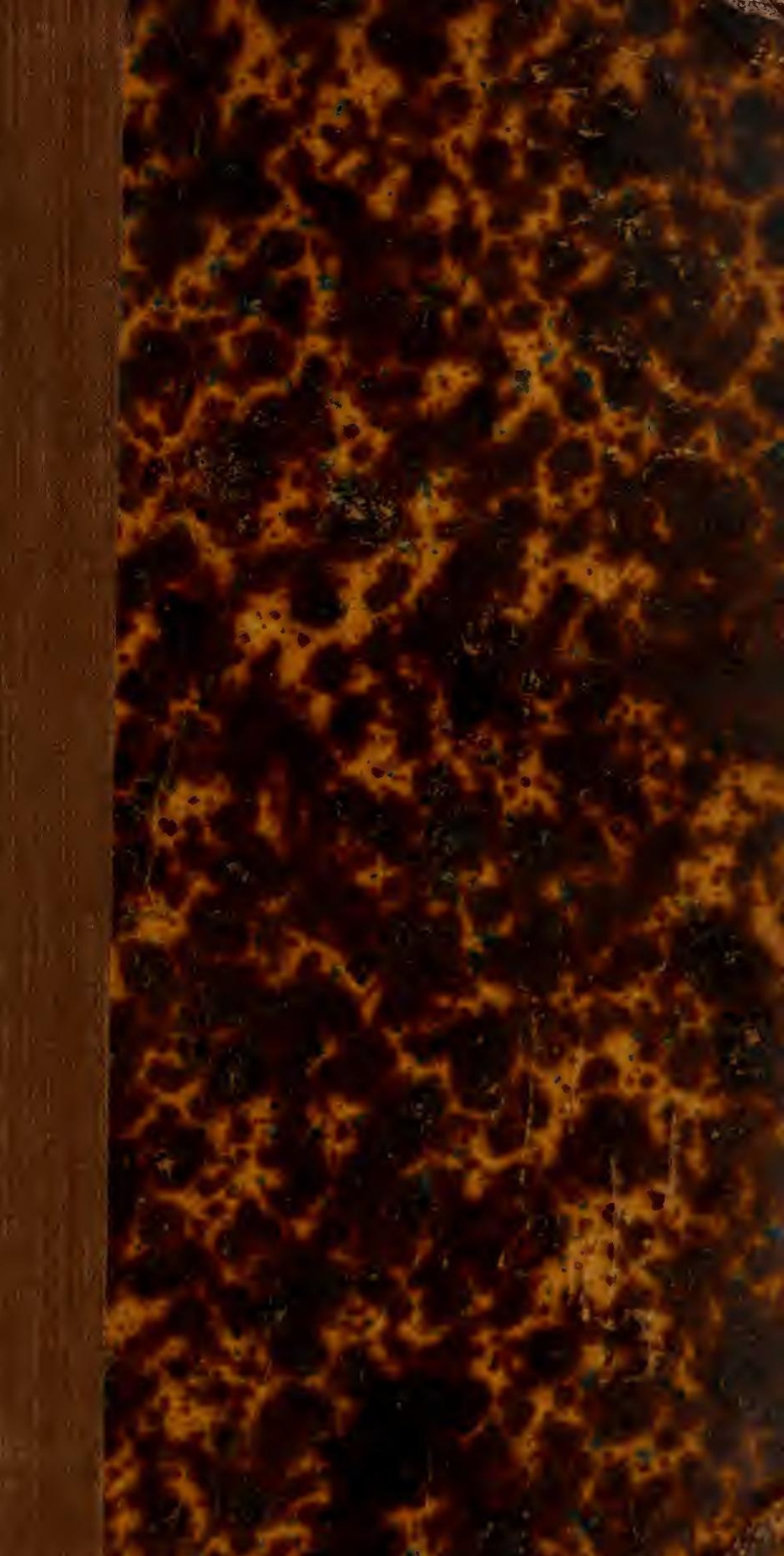



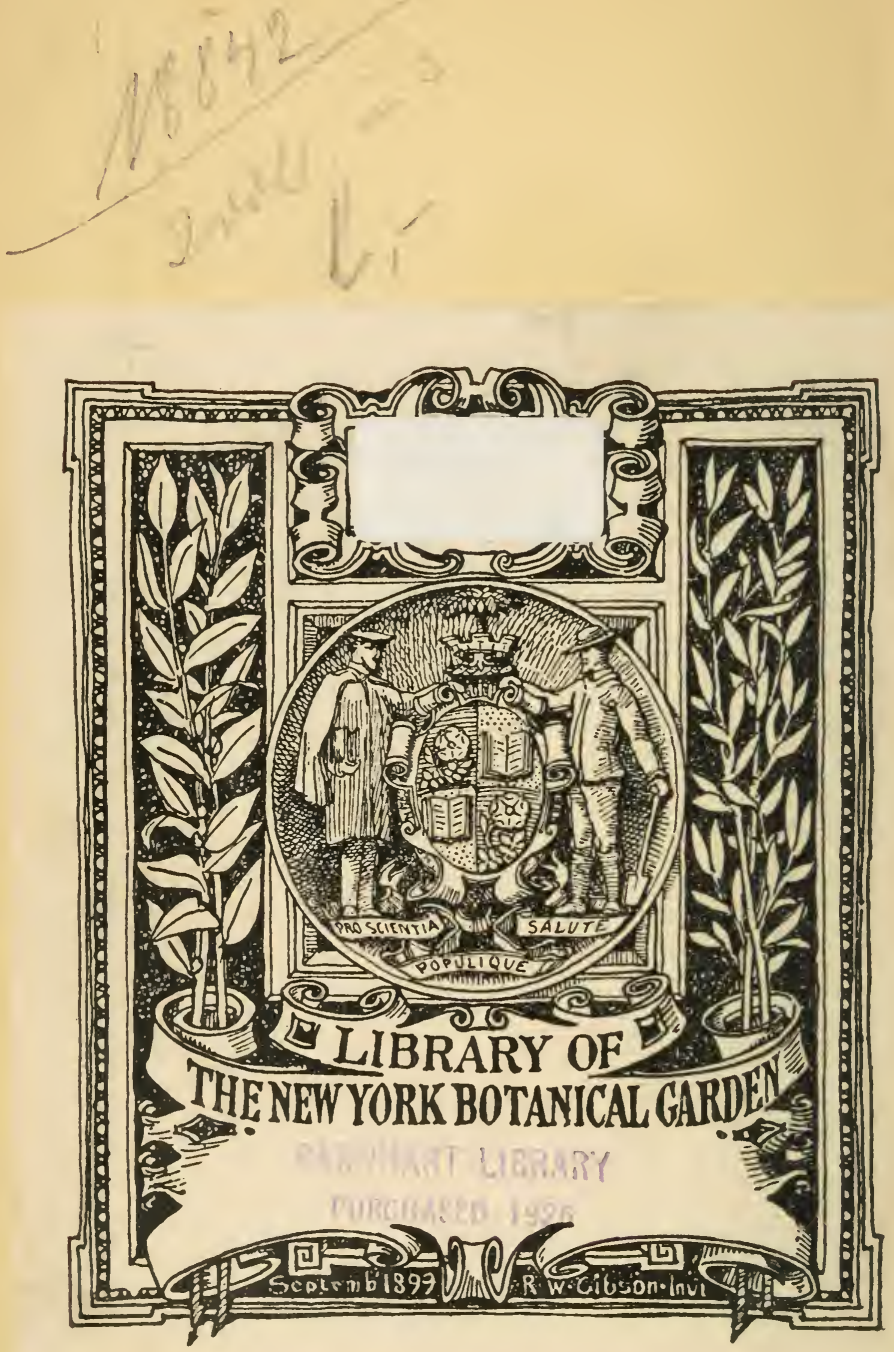
$\cdot$ 



\section{CAROLI LINNAEI}

EQVITIS DE STELLA POLARI,

ARCHIATRI REGII, MED. ET BOTAN. PROFESS. VPSAL. ACAD. VPSAL. HOLMENS. PETROPOL. BEROL. IMPER. LOND. MONSPEL. TOLOS. FLORENT.'SOC.
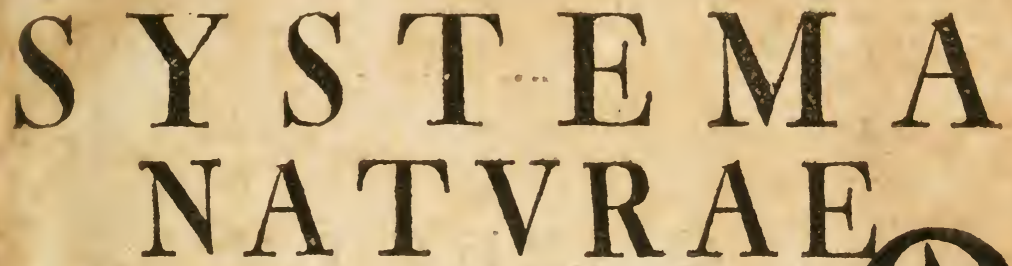

REGNA TRIA NATVR SECVNDVM C LASSES, ORDINES, G E N E R A, S P E C I S, C V M

CHAR A CTERIBVS, DIF FERENTIIS, SYNONYMIS, LOCIS. TOMVS I.

\section{PRAEFAT V E T T}

IOANNES IOACHIMVS LANGIVS

MAT H. PROF. PVBL, ORD. HALENS, ACAD, IM P, ET BORVSS. COLLECA,

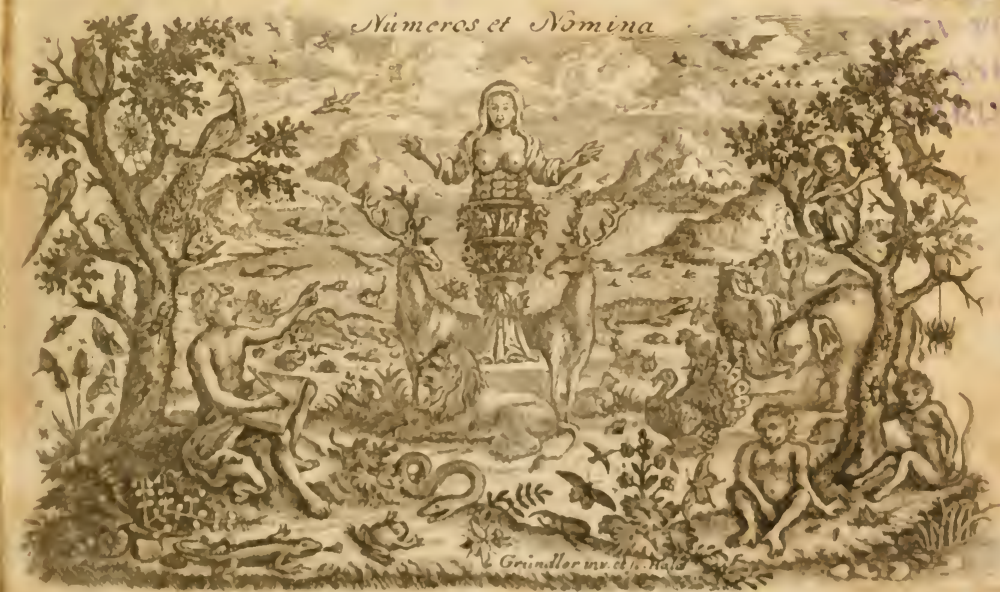

AD FDITIONEM DECIMAM REFORMATAM UOLMIENSEM.

HALAE MAGDEBVRGICAE

TYPISETSVMTIBVS IO. IAC. CVRT. M NCSLY⿱. 


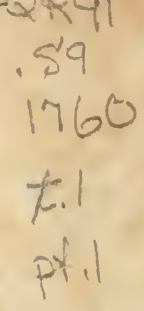

\section{O I E H V A}

Quam ampla fint Tua Opera!

Quam Sapienter Ea fecifir!

Quam plena eft Terra poffefrone Tua! 
27 Jacchus - lege Jacc us.

39 graius gra is

46 barbata bar ba a

so Jaiacu Jajacu

63 Jaculus Jaculus.

87. Percnopterus - perenopterus.

95: iocosus jocosus.

100. caeruleocephalus caerulocephatus.

112. Jynx

114. maior

Jynx.

140 Aiaca

major.

142 ignea

221. Naia

tiaja.

225 HGaie

migra.

Naja.

220 iaculus

Hodje.

23, Raia

231. Oxy.chy nchus

263. Jo20

Jaculus.

284 Julis

311 . Scyrhynchuo

325 Yeses Raja.

Seyrinchus.

Jaxo.

Julis.

Oxyrinchus.

326 Bioerkna

Jeses.

336 gyngnatus

Bjoertina.

Syngmatires.

348 hbenorhoidalis

haemorchidalis.

354 parallelepipedur

- parallelipipedus.

3 ss polygraphus

- poligraphus.

364 iamaicensis

jamaicensis.

$36 \mathrm{~g}$ harmorrhoidalis

hacmorrhoidalis.

370 hypochaeridis

385 chloropus hypochaeriois.

3 g 6 baiulus cloropus.

412 erythrocephalus bajulus.

erytrocephalus.

419 maialis

445 Juniperimuo

majalio.

452 Jacear

Yuniperinus.

45\% Jumiperina

Jaceas.

464 Eurippylus

Tuniperime.

465 Aglaia

curupylus.

$4>5$ Iurtina

crglaju.

4 y5 Janira

Yurtina.

481 Anglaia

Yunira.

$485 \mathrm{Jason}$

Iglaja.

487 Cencus

Yason.

500 Caia

Q Qmens.

573 Yota

Caja.

Jota. 
527. Jamata

531 . Löflingi:na

542 Raiella

544 . inncea

565 iaculator

567 Jumiperi

s'os Yuniperina

sog plebia

598. aeginoctialis

613 Ho aematoporis.

618 . litonalis

$62 \mathrm{~g}$ Nocia

$63 \mathrm{~g}$. Juhus

6g6. Jacobaca

711. Siphunculus

720 Avris Indae

772 Janthina

794 labyrintiformis

\$12 Tua lege Janata.

Coeflingiana.

Rajella.

juncea.

jaculator.

Juniperi.

Huniperína.

plebeja.

aequinoctiatis.

Hoamatopi. littoralis.

Naja.

Julus.

Jacobaea.

Qipuncentus.

twis Juove

janthisea.

labyrinthiformis. Uva.

Haec omia nomina a me covrecta sunt ad unum exemplar verae Editio", nis Decimae Hoohniensis (MDCCLVIII) in qua Carolus Limarus prime Bino," menclaturam fundavit.

A.C.Oudemans. Joun.

Loil. \& Bot. Cand.

Frajectum ad Rhemum.

Die $X X$. mensis Aprilis

MDCCCLXXX III. 
S:AE R:AE M:TIS SVMMAE FIDEI VIRO CELSISSIMO ILLVSTRISSIMOQVE C O MI T I

\section{D. ${ }^{\mathrm{NO}}$ CAR. G. TESSIN,}

REGIS REGNIQVE SVIOGOTHICI SENATORI, REGIAE CANCELLARIAE PRAESIDI,

AD AVLAM REGIARVM CELSITVDINVM SVMMO MARESCHALLO,

EDVCATIONI SERENISS. PRINCIPIS GVSTAVI PRAEFECTO; CANCELLARIO ACADEMIAE ABOENSIS,

ORDINIS S:AE R:AE MAIEST. SERAPIIINORVM ERVITI AVRATO ET COMMENDATORI, EQVITI AVRATO DE ACVILA NIGRA, NEC NON OMNIVM S:AE R:AE MAIEST. SVEGIGAE ORDINVA CANCELLARIO

\section{S A C R V M.}

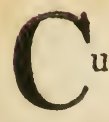

um quiuis auctor fperet fe apud pofteros gratiam habere et poffe fecum duratura nomina educere, AVCTOR I meae fortunae Libellum decimo nunc offero; ILLE me, peregrinum in patria, reducem excepir; ILLE mihi Stipendium ab Ordinibus regni expeciit; ILLE mihi fpartam Medici claffici procurauit; ILLE mihi Munus, quo fungor, conciliauit; ILLE mihi Titulum, quo diftinguor, parauit; ILLE me ad Sereniff. Reges introduxit; ILLE meas errare boues vt cornis et ipfum ludere quae vellem calamo permifit agrefti;

quare

cama prius gelido difint ab/inthia ponto quam inmemor viliam

\section{TANTI MAECENATIS}

Vpfaliae 1757 , d. 24 Maii.
Cultor ceuntifinus

C. LIN NAEVS。 


\section{Magnus eft DEVS nofter, \\ et magna eft. potentia Eius, \\ et potentiae Eius $n 07$ eft mumerus.}

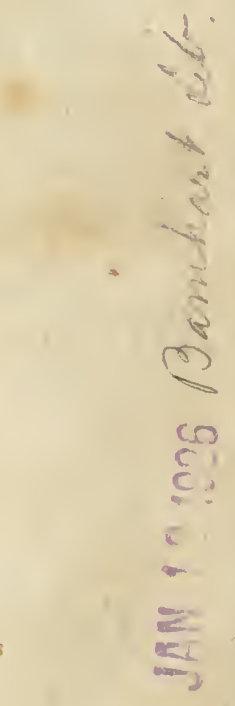




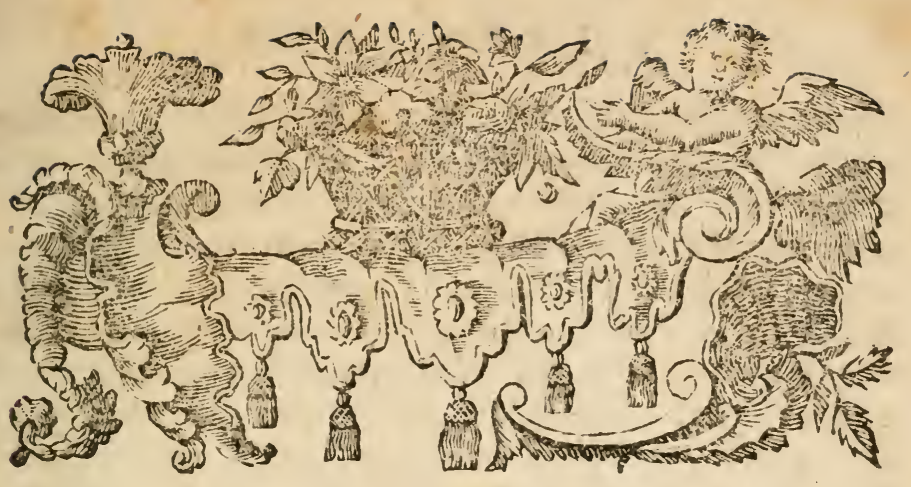

\section{NATVR ALISTIS}

\section{IOANNES IOACHIMVS LANGIVS.}

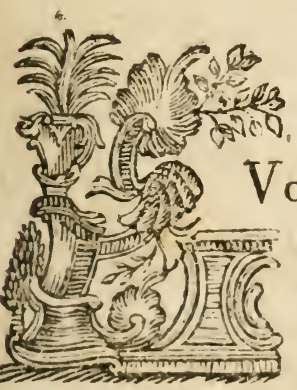

IIA:2UBH

Vos compello, et denominatione hac, vobis honcrifica, defigno, qui in Hiforiae naturalis ftudio ita verfamini, ve in cognitione rerum natura lium acquirenda et augenda non circa fingularia et indiuidua dignofienda atque ficut vfus fert denominanda haereatis, fed fubiecta haec fecundum conuenicntian et differentiam noffe cupiatis. Ita enim zarietates a Jpeciebus, has a gencriúus caute diftinguere, in ordines et claffes digerere atque notiones has adacquatis defcriptionilus, appellationibus et terminis tcchnicis munire gaudetis. Vobis offertur omnium fub= icctorum, quae ex tribus naturae regnis innotuerunt, ads nequata, concinna, diftincta defcriptio: imo, quod vix optari, nedun exfpectari poterat : habetis non claflum folum et ordinum; fed et genertum atque fpecicium

\section{NVMEROS ET NOMINA.}




\section{P R A E F A T IO.}

Tanto maius pretium huic labori eft conftituendum, quo magis expedita via nunc per ampliffima haec regna patet. Omnes hactenus in lucem protracti thefauri naturae hic in compendio quidem, non parca tamen manu, offeruntur. Fructum hunc debemus ftupendo illi labori et follertiae nunquam fatis mirandae, qua fummuS LIN N AEVS in fuam poteftatem redegit haec omnia nobisque tradidit. Et quam multae operae in hoc negotio exantlatae noftrum confpectum fugiunt? Non vnum enim Augiae ftabulum Herculi huic fuit repurgandum. Erat tempus, de quo Virgilius canit:

Nauita tunc ftellis numeros et nomina fecit.

Noffer Eques de ftella polari, dum animalibus, vegetabilibus, mineralibus

\section{numeros et nomind fecit,}

ftellarum, quae videri, diftingui ct numerari poffunt, numerum et nomina multoties fuperauit. - Labor vero is', quo characteres obferuantur, fubietta aftinia affinibus comparantur, in ordinem concinnum rediguntur, defcriptionibus et appellationibus ornantur, longe illum fuperat, quo nauita feciffe dicitur.

\section{Numeros et nomina.}

Vel meo exemplo intelligo, quanta fint perilluftris INNNAE I in ftudium Hiftoriae naturalis merita, et quanta alacritate hunc laborem fufcepturi fint naturaliftae. Mihi valde iuueni obuiam quafi iuerunt petrefacta agri Halenfis er Mansfeldenfis, inuitarunt me, vt lapidicinas et fodinas xylanthracum, lithantracum, fchifti cupriferi, atque officinas fuforias et eliquatorias in vicinia luftarem. Haec vifa ad ftudia academica et fcientiam imprimis naturalem accedentem inuitarunt, vt in metalli fodinas Saxoniae, Bohemiae, Herciniae, Thuringiae defcenderem. Nulla ex eo tempore dies erat fine linea, apparatus rerum naturalium, indies angebatur, ftudium fubiecta haec diftincte cognofcendi cum mole et numero crefcebat. Tales eo magis defiderabam defcriptiones, quales Botanici, de re- 


\section{P R A E F A T I O.}

gno vegetabili dederunt, quo lubentius ego, muneri docendi publico admotus, iuuenum animos ad haec ftudia incendere, incenfos erudire cupiebam, praefertim cum anno 38 videre mihi contigerit L INNAE I noftri Genera plantarum. - Quoscurque fcriptorum antiquorum, mediorum, recentiorum confulebam, illi me multis fynonymis, homonymis, defcriptionibus obfcuris, incompletis ambiguis onuftum, vera et diftincta cognitione vacuum, dimittebant: vt faepius dicendum mihi fuerit:

\section{Feciftis probe:}

\section{Incertior fum multo, quam dudum.}

Ipfe tentabam, cum anno 35 Elcmenta pbilofophide naturalis edidiffem, lapides hos mouere, chemiae principiis in fubfidium vocatis. Cum vero anno 38 I). I. I. LAW S ON in itinere fino me inuiferet et viderem Sy/tema naturae, quod folo nomine ex praefatione Generum plantarum noueram, ftatim mentem meam in tantum mutaui, vt magnum hunc architectum fequi maluerim, quam nouum ftruere aedificium, atque de edendo apud nos et fimul in germanicam linguam transfundendo hoc fyftemate follicitus fui. Lectiones meae in Hiftoriam naturalem inftitutae mihi ipfi et auditoribus meis multo magis factae funt incundae et proficuae dum Tantum fequeremur ducem. Vix dici poteft quantum hinc anćtus fuerit amor in Hiftoriam naturalem, plures inftitutae funt rerum naturalium collectiones, ex meis his, vel potius Magni LINNAEI, difcipulis enaferunt in fcholis et gymnafiis quibusdam doctores Hiftoriae naturalis. $\mathrm{Ea}$ apud nos fieri coeperunt, quae ve fierent I. BR O W A L LV S in Difcurfu de introducenda in Scholas et Gymnafun Hiforiae naturalis lectione, qui Criticae Botanicae iunctus eft, optanit. Aucto ita difcentium numero augebitur et docentium numerus, atçue ita ftudium hoc, quod fieri poteft et debet, tandem vniuerfale fiet. Nec vanum effe hoc augurium, res ipfa loquitur. Poft primam enim editionem Lugdunenfem huius fyftematis habemus 


\section{PRAEF A T IO.}

extra Sueciom alteram Lugdunengem, duas Hatlenfes, Lipfienfem vnam, Parifinam vnam. Ipfe Perilluftris antctor iteratis editionibus Holmienfibus incrementis Hiforiae naturalis ita profpexit, vt nunc exhibuerit decimam editionem, quam appellauit reformatam, nos denominationem locupletiffinae editionis iure meritoqueaddimus. $\mathrm{Ea}$, quibus haec editio decima habet prae reliquis praecipua, vel ex ipfius molis augmento fpectator aeftumare poterit, attentus lector antem longe plura, eaque fummo ftudio in compendium redacta reperiet, quam ipfa moles polliceri videbatur. Noftrum non eft ea hic recenfere, quae ad naturam ipfam reformata, quae de nouo addita funt. Videantur abunde in ipfo opere, quae breuiter delineata funt in Ratione Editionis. Quae cum ita eflent, nec tamen fatis diu valde defiderata haec editio apud nos, et vix nifi valde magno pretio haberi potuerit, non potui non inftitutum typographi noftri, Viri eximii I. I. Curt, de recudenda apud nos hac ipfa editione decima probare; praefertim cum ex primis plagulis mihi oblatis intelligerem; eum nullis fumtibus, nulli operae parcere, fed operam dare vt noftra editio Holmienfi nitore chartae et elegantia typorum minime cederet. Nec difplicuit confilium typographi de addenda titulo operis fculptura ad rem ipfam valde appofita. Ante omnia vero commendaui vt, quam fieri poffet fine mendis haec editio exemplar Holmienfe referret. Quam hoc in tot nominibus, in tot numeris fit difficile, aequi rerum arbitri intelligunt. Puto tamen meam, quam in plurimis plagulis hac in re, quantum per reliquas occupationes licuit, adhibui operam, pauca praeteruidiffe, tempeftiue indicanda. Vale, lector beneuole, et his, tanquam naturalifta, nomine hoc dignus, ita vtere, vt gloria in auctorem naturae fummum, vtilitas in publicum redundet, quem Tibi ipfe I I N AEVS Hiftoriae naturalis commendauit finem et vfum. Dabam in Regia Fridericiana Halenfi, menfe $\mathrm{Fe}$. bruario Anni M DCC LX. 




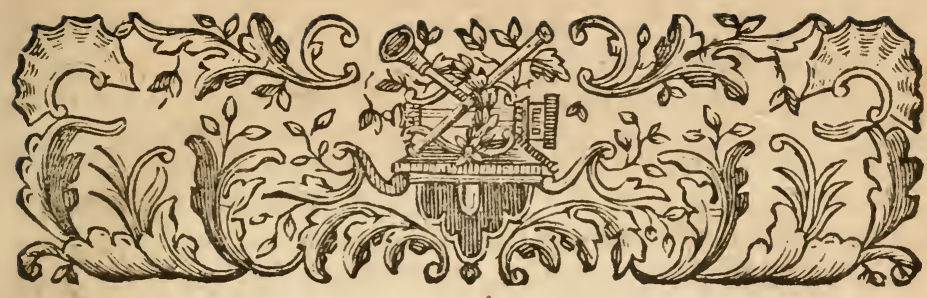

\section{R A T I O E D I T I O N I S.}

SYSTEMA NATVRAE iteratis vicibus prodiit.

+ I. Leydae 1735 Folio imper.pag. I2. a me per I. F. GRONOVIIVM. Conjpectus tantum operis et quafi Mappa Geogr.

2. Holmiae I740 O\&tauo. pag. 80. a me ipfo reuifum. Auctum Animalium nomenclaturis, cbaracteribus.

3. Halae 1740 Quart-oblong. pag. 70. per I. I. LANGE. Latino-germanice, caeterum idem cum I.

4. Parifiis I740 O\&tauo. pag. IO8. t. I. per B. JVSSIEVM. Adiecta nomina Gallica. idem cum 2.

5. Halae 1747 Octauo. pag. 88. per M. G. AGNETHLER. Nomina Germanica, caeterum idem cum 2.

† 6. Holmiae 1748 Odtauo. pag. 232. t. 8. auctum a me ipfo. Chavaderes effent. Vegetabilinn, Species Anim. et Lapid.

7. Lipfiae 1748 O\&tauo. pag. 232. t. 8. per Typographum. Nomina Germanica, cacterum idem cum 6.

8. Holmiae 1753 Octau. p. I36. Vegetabilia per I. I. HAARTMAN. At regnum Lapideum Scarae 8 vo per Möller.

+ 9. Leydae 1756 Odtauo. pag. 226. per GRONOVIVM. Panciffrna de Auibus, Pifcibus. idem cum 6.

+ Io. Holmiae hac edirione, longe auctius factum a me ipfo:

Specics animalium omnium mihi cognitorum cum Differentiis, Synonymis, Locis, nominibus Triuialibus.

Methodus Animalium ex nouis obferuatis reformata.

Pifcium imprimis noua methodus cum Auctorum obferuatis radiis pinnarum.

Plantaruns reformati charąteres, emendatae differentiae variae.

Lapidum plures fpecies, obferuationes, et omnium Nom. Triuialia.

Objerrationes in Regna Naturae nouae, cum introductione in Naturam. 
COLLECTANEA, quae obtinere potui, difpofui ; imprimis adiuuarunt

S: I ADOLPHI FRIDERICI Regis Mufeum tom. I. quem edidi, et tom. 2. $M \int c$. de Animalibus exoticis, quem compofui.

S:ae lovisae vlricae Reginae Mufeum $M j \dot{c}$.

in Concbiliis et Injectis ftupendum, defcriptionibus adornaui,

c. TESSIN Mufeum. Holm. 1753. Fol. in Lapidibus et Conchiliis magnificum.

c. DE GEER Mufeum.

Amplibiis, Infect is aliisque rarioribus diues.

vPSALIENS Is Mufeum Academise:

ex Animalibus vndiquaque conquifitis.

STOCKHOLMENS IS Mufeum Academiae Scientiarum.

Variis pulchris naturalibus fcatens.

IT INERA propria: Oelandicum 174I, Gatlandicum I74I, Wes flrogatbicum 1746, Scrnicum 1749, edita; et Lafponicun 1732 , Dalekarlicum 1734 inedita.

Difcipulorum quondam meorum inftitura Itinera, mihi, ab exterorum gazophylaciis remoto, proficua:

C. TERNSTROEM in Afam (fed periit in Poulicandor) 1745 .

r. KALMII in Penfyluanian et Canadam. 1747.

3. MONTIN I in Lapponiam Lulenfem. 1749.

F. HASSELQVIST in Aegyptum et Palaeftinam. I749.

O. TORENII in Malabariam et Suratte. 1750 .

P. OSBEGXI I Chinam et Iauam. 1750.

T. LOEFLING1 in Hifpaniam et Americam. I7jI.

P. I. BERGII in Gotlandiam infulam. 1752.

M. KAEHLERI in Italium et Apuliam. 1752.

D. SOLANDRI in Lapp. Pitenfem et Tornenfem. 1753.

D. ROLANDRI in Surinamum et Euftatium. 1755 .

FINEM Operis obtinuero, $\mathrm{fi}$ inde

Peregrinatores dignofeant notiora antea naturalia.

Curiofi attentiores Mufea intrantes, vifa intelligant.

Docti noua, nec trita, actis fuis inferant.

Scientia facilius augeatur fepofitis vulgatioribus.

Nonina magis ftabiliantur, imprimis Trinialia et Generica,

His vtere L. B. donec alii plura praeftantioraque fiftant.

Vpfaliae 1757 . Maii 24.

Docuifi me DEVS a inuentute mea,

et vsque nunc pronunciabo Mirabilia Tua. 




\section{N T RO I T V S.}

TOMO mundi intraturus theatrum, quaeritur Quis fit: 11 vnde ortus? e ftemmate Creationis; Quo tendut? ad vitam beatam; Quid hic? intueri naturam; Quo munere? curiofum effe fimilemque.quidem reliquis animantibus (quae vorant, ludunt, pruriunt, generant, multiplicantur, dormiunt, commoda ftabula quaerunt, proximis fui generis inferuiunt, vitam tuentur, fentiunt, percipiunt,) fed nobiliorem, vtpote qui meditatur, ratiocinatur, miratur pulchrum fapientis opus Artificis. O quam contemta res eft homo, nifi fupra humana fe erexerit! Quid enim erat cur in numero viuentium fe pofitum gauderet? an vt Cibos et Potiones percolaret? ve hoc corpus cafurum periturumque farciret? quae tanta neceflitas in fundum telluris intimae hominem merfit, vt oblitus dierum, oblitus fortunae melioris, ab hoc fe auerteret? Puto certe multos ad fapientiam peruenturos, nifi fuperbia magnae fortunae inflati, putaffent fe perueniffe; fapiens vtique eft, qui fines repicit.

Finis Creationis telluris eft gloria DEI cx Opere $\mathrm{Na}$ turae per Hominem folum.

Tanto igitur magis noffe Naturam operae pretium, quo nullum maius eft! neque enim quidquam habet in $\mathrm{fe}$ huius materiac tractatio pulchrius, cum multa lateant futura vfui, quam quod hominem Magnificentia fua detinet, nec mercede, fed Miraculo colitur!

Quota enim pars operis Tanti noftris oculis committitur, et quam multa, praeter haec, quae vidimus, in fecretum eunt nunquam humanis oculis orientia; neque enim omnia DEvs humanis oculis nota fecit. Multa etenim funt quae effe audiuimus, qualia autem fint ignoramus! quamque multa venientis aeui populus, ignota nobis, fciet! multa feculis tunc futuris, cum memoria noftri exoleuerit, referuantur; veniet tempus, quo ifta quae nunc latent, in lucem dies extrahat et longioris aeui diligentia. Rerum enim Natura facra fua non fimul tradit; initiatos 
nos credimus, in veftibulo eius haeremus; illa arcana non promifcue, non omnibus patent! redußa et interiore facrario claufa funt; inuoluta veritas in alto latet.

$\mathrm{Si}$ in hoc iuuentus fobria incumberet $\left({ }^{*}\right)$, hoc Maiores docerent, hoc Minores addifcerent, vix tamen ad fundum veniretur, in quo veritas pofita eft, quam nunc in fumma Tellure, et leui manu quaerimus. Magni omnino res eft rerum Naturae latebras dimouere, nec contentum exteriore eius confpectu, introfpicere et in Diuina fecreta delcendere. Seneca.

Intraui itaque denfas vmbrofasque Naturae Siluas, hinc inde horrentes acutiffimis et hamatis fpinis, euitaui quotquot licuit plurimas, at neminem tam effe circumfpectum didici, cuius non diligentia fibi ipfi aliquando excidat, ideoque ringentium Satyrorum cachinnos, humerisque infiliéntium fimiolorum fpeculationes fuftinui, incefli viam et quem dederat ${ }^{\star}$ curfum fortuna peregi.

\section{O I E H O V A !}

Quam magnifica funt Tua Opera!

Vir infipiens non cognofit ea

et ftultus non animaduertit ea.

\section{David.}

(*) Confer Worm. mulf. praefat. I. 




\section{$\begin{array}{llllllll} & \mathrm{I} & \mathrm{P} & \mathrm{E} & \mathrm{R} & \mathrm{I} & \mathrm{V} & \mathrm{M}\end{array}$ N A T V R A E.}

D E V M fempiternum, omnifciun, omnipotentem a tergo tranfeuntem vidi et obftupui! legi aliquot Eius veftigia per creata rerum, in quibus omnibus, etiam minimis, vt fere nullis, quae Vis! quanta Sapientia! quam inextricibilis Perfectio! Obferuaui Animalia inniti vegetabilibus, Vegetabilia terreftribus, Terreftria telluri; Tellurem dein ordine inconcuffo volui circa folem, a quo vitam mutuatur; Solem demum circa axin gyrari cum reliquis Aftris, fyftemaque Siderinn, fpatio et numero vix definiendum, mediante motu in vacuo nihilo fufpenfum teneri ab incomprehenfibili Monente primo, EN TE ENTIVM (a), Caufja cauffarum, Cuffode Rectoreque vniurrfi, mundani buius operis Domino et Artifice. Vis illud F Aт $\vee$ M vocare, non errabis, eft ex quo firpenfa fint omnia. Vis illud N A T V R A M vocare, non errabis, eft ex quo nata fint onnia. Vis illud $\mathrm{P}$ R O VI DEN T I A v vocare, recte dices, eft cuius confilio mundus actus fiuos explicat (b); totus eft S ENSVS, totus V I S V , totus AVDIT VS, totus A N IMAE, totus A N1M1, totus SV1; buitus EXTERA indligare non capit bumanae coniectura mentis (c); $\mathrm{N}$ V M E is effe credi par eft, aeternum, innmenfunn, neque genitum neque creatum (d). Hoc fine quo nibil eft, quod totum boc fundanit et condidit, quodque oculos noftros et implet et effugit, cogitatione tantum vifendum eft; in fanctiore enim fecelfu Maieftas tanta delituit, nec villi dat aditum nifi animo. (e)

MVNDVS complectitur omnia fub ioue, quae in notitiam noftram per fenfus cadere poffunt; funt haec Aftra, Elententa, Tellusque inenarrabili velocitate circumacta; borum innoffenfanm velucitaten procedere videmus asternae legis imperio, nec effe temere errantis bunc ordinem, neque quae temere coinerunt tanta arte pendere, vt terravum grauiffimum pondus $\int e-$ deat (quaji) innmotum et circun $\int_{e}$ properantis coeli fugans jpetct. (f)

(a) Ariftotcli. (b) Senec. quaeff. II: 45. boc refpectu, fed caute, ne effectus fumatur pro caufia. (c) Exod. XX: 4, (d) Plin, Nat. II. t.7. (e) Senec. VII: 3 . (f) Since. $I: l$, 
ASTRA funt remotiffima corpora lucida, quae gyrantur motu perpetuo; funt haec aut S I DER A propria luce radiantia, vt Sol, remotioresque Stellae fixaie; aut P L A N E T A E a fideribus lucem mutuantes, quorum primarii Solares: Saturnus, Iupiter, Mars, Tellus, Venus, Mercurius; fecundarii obfecundantes Planetis, ve Luna Telluri, aliique; nec poffe fine Cuftode tantum opus ftare, nec bunc fiderum difcurfum fortuiti inpetus effe, nam quae cafus incitat faepe turbari et cito arietare. (g)

ELEMENT A funt corpora fimpliciffima, atmofphaeram Planetarum conftituentia, fpatis inter Aftra forte replentia:

TERRA opaca, fixa, frigida, quiefiens, fterilis. A QVA diapbana, fluida, bumida, penetrans, concipiens. A ER pellucidus, elafticus, ficcus, obuolitans, generans. I GN IS lucidus, refiliens, calidus, euolans, viuificans. Omnium Elementorum alterni recurjus funt, quidquid alteri perit in alterum tranfit; alternae funt vices revum. ( $h$ )

TELLVS eft globus planetarius horis 24 rotatus, circum folem quotannis in orbem adus, Elementorum atmofphaera obvelatus, rerum Natuvalizm ftupendo cortice te\&tus, cuius cognofcendae fupe:ficiei ftudemus. Globus hic terraqueus eft, cuius depreffiorem partem Aquae inundant et Mare lente coarEtandum; elatiorem vero aquae fugiunt fenfim dilatandam in Continentem ficcam habitabilem. Haec Aquarum halitu, vi Aëris in nubes acto, irroratur, vt fummi montes perennique niue Alpini Scaturigines in Flutios perennes concurrentes, eamque permeantes, potum terrefti cibo addant in alimentum incolarum, dum Venti motum excitant Ignis calore viuificatis corporibus. Sic omnia adiunabunt Natmam, vt naturae opera pe. ragantur (i).

N A TVRALIA funt corpora cuncta Creatoris manu compofita, corticen Te!luris conftituentia, in Regna Naturae tria diuifa, quorum limites concurrunt in Lithophytis.

L A P I D E S corpora cungefta, nec viua, nec fentientia.

VEGETA B I L I A corp. organifata et viua, non fentientia.

A N I M A L I A corp. organifata et viua et fentientia, fponteque fe mouentia. Tota enim buius mundi concordia ex difcordibus conftat ( $k$ ); Nec ad vnam Natura forman opus fum praejtat, fed in ipfa varietate $\int e$ inctat (1).

HOMO

(g) Senec. (1) Senec. DII: $=7$. 


HOMO fapiens, creatorum operum perfectiffimum, vltimum et fummum, in Telluris cortice, Maieftatis Dininae ftupendis indicis obtecto, conftitutus, fecundum fenfus iudicans artificium, admirans pulchritudinem, veneraturus Auftorem. Retrogrediendo per generationes multiplicatas videt Creatorem; Progrediendo per conferuationem multiplicandam videt Naturam; ad virumque inuitant Pulchritudo, Mechanifmus, Nexus, Fines, Commoda. Sic totus Mundus gloria diuina plemus eft $(\mathrm{m})$, dun ommia creata opera Deum glorificant per bominem ( $\mathrm{n}$ ), qui ex inerti humo, Dei manu viuificata contemplatur auctoris fui Maieftatem ex fine creationis, dignus hofpes conftitutus Summi Entis praeco. Haec contenepatio Naturae elladit praeJentia voluptatis cocleftis, cuius particeps Animus in luce obambulat et tanquam in terreftri coelo vitam degit (o); Nec pictas aduerfius. DEun, nec quanta buic gratia debeatur, fine explicatione Naturae intelligi poteft (p).

SAPIENTIA, diuinae particula aurae, fummum eft attributum Hominis Sapientis. Primus Sapientiae gradus eft res ipfas noffe. Notitia confiftit in vera idea obieforum, qua fimilia a diffimilibus diftinguuntur notis propriis, a Creatore rebus infcriptis; hanc notitiam vt cum aliis communicet, nomina propria non confundenda fingulis diuerfis imponar; Nomina enim fi pereunt, perit et rerum cognitio. Haec literae elementaque erunt, quibus defticutus nemo naturam legerit; quum ignorato genere proprio, nulla defcriptio quamuis accurate tradita, certum demonftret, fed plerumque fallit (q).

METHODVS, anima Scientiae, fiftat notas proprias rebus inditas fyftematice digeftas; Syftema apte quinquies tantum. fubdiuidituz: fic
Clafis,
Ordo,
Genus,
Species, Varietas.

Genus fun. G. intermedium, G. proximnm, Species, Indinidunm. Prouinciae, Territoria, Parocciae, Pagi, Domicilium. Legiones, Cobortes, Manipuli, Contubernia, Miles. Nifi enim in ordines redigantur ct veluti caftrorum acies difribuantur, tumultu et fuctuatione onnia perturbari neceffe eft ( $\mathrm{r}$ ).

NOMINA refpondeant Methodo Syftematicae; fint itaque:

Nomina Claffum, Ordinum, Generum, Specierum, Varietatum, Charąter Clafium, Ordinum, Cienerum, Specierum, Varietatumq: DIfferentiis definita, nam nomina noffe oportet qui rem fcire A 4 velit,

(in) Efa. VI: 4.

(n) Dauid. CXVIII.

(o) Aส?. bolm.

(p) Cisero.

(q) Caefalpirus.

(r) Caefalpinus, 
velit, confufis enim nominibus onnia confundi neceffe eft. Nominum ideoque impofitio primi bominis in aurea aetate actio erat.

SCIENTI A Naturae innititur Cognitioni Naturalium Methodicae et Nomenclaturae Syftematicae tamquam filo ariadneo, fecundum quod Naturae maeandros vnice tutoque permeare liceat.

Ordo alius fruentis eft, alius eft inhabitantis. Creator incepit a fimplicifimis elementis Terreis, per Lapides, Vegetabilia, Animalia in homine defiit. Homo autem incipiat a fe ipfo, definat in terra. Auctor Syftematis a particularibus ad vniuerfalia adfcendat; Doctor vero contra a generalibus ad fpecialia defcendat. Fontes enim concurrunt in riuos, hi in fluuios; adfcendat autem nauta quousque poterit, nec tamen fontium vltimas origines attigerit. Data diftinEta rerum cognitione vlterius penetrandum in fpecialifilimas Proprietates, Phaenomena, Myfteria, Dotes, Vires, Vfus; Naturalis quum Scientia trium regnorum Fundamentum fit omnis Diaetae, Medicinae, Oeconomiae tam priuatae quam ipfius naturae. Felices agricolae bona fi fua norint.

Omnes res creatae funt diuinae fapientiae et potentiae teftes, diuitiae felicitatis humanae; ex harum vfu bonitas Creatoris; ex pulchritudine Sapientia Domini; ex oeconomia in Confersatione, Proportione, Renouatione, potentia Maieftatis elucet. Earum itaque indagatio ab hominibus fibi relictis femper aeftimata; a vere eruditis et Sapientibus femper exculta; peruerfe doctis et barbaris femper inimica fuit.

R E-

Narrabo mirabilia Tua DOMINE, et virtutem: Terribilium Tuorum dicant generationes. Dauid. 




\section{REGNVM ANIMALE.}

\section{REGNVM \\ A NIMALE.}

ANIMALIA organifatione viua, neruis fentiunt, percipiunt, feque ex arbitrio monent motu poffibili.

VIVENTIA fingula, in multiplicatione prodiga Natura, orditur a minimis, generat in fluido, incipit in oui liquido, cum onne vinum ex outo.

OVVM intra Tunicas, faepe includentes Albumen, continet $V i$ tellum, cuius lateri cmergenti infertum Punctum faliens, vegetans in Embryonem, caulefceniem Funiculo vmbilicali, radicatum placenta vitellina.

MATER prolifera promit ante generationem viuum compendium noui animalis fuique fimillimi, tamquam plumulam intra femen vegetabile; hoc Patris polline fpermatico electrifatum calore excluditur; Punctum faliens enim Oui incubantis Auis oftendit primum Cor micans Cerebrumque cum Medulla; cerculum hoc, ceffans a frigore, excitatur calido halitu, premitque Bulla ä̈rea, fenfim dilatata, liquores fecundum canales fluxiles. Puncium vitalitatis itaque in viuentibus eft tantum a prima creatione continuata vitae ramificatio, cum ouum fit gemma matris $a b$ exordio viua, licet non fentiens ante foecundationem; fic $G_{e-}$ neratio Aequinoca nulla.

CORPVS Animantium compofita eft machina:

NATVRAlis, vegetat e cranii Tubere cruftato, Caule verticali, articulato, rigido, oppofite ramofo, cui infident Folia carnofa, fibrofa, fparfa, apicibus etiam affixa in Mufculos, prodeunte Fructificatione genitalium e dichotomia vltima caulis.

vitalis, e Bulbo Cordis perpetuo mobili, radicata vafis laEteis intra tubi inteftinalis fterquilinium, ramificatur ad caulem naruralem duplici canali circulante, ne fuccus alimentaris quauis tempeftate et ftatu deficiat.

AN1MAL1S, e Bulbo Cerebri repentisque Medullae, indolis incognitae, fedis cogitantis, Fila fimplicifima, eleetrica ad fibras omnes irritabiles exferit, per quae cogitans fentit et mouet.

ORGANA fenfuum machinae funt phyficae, inferrae extremitati nerui, fenforio cerebri proximi, quibus diuina arte percipit Animal:

ocvlvs: Camera obfcura imaginem proportione, figura, colore depingens. 
A v R IS: Tympramu membranae chorda tenfae fuper cochleam, a motu aetheris tremens.

NASvS: Menzbrana latıfima, humida, contortuplicata, nëris perreptantis volatilia figens.

LiNGva : Spongiolae bibulae, fparfue, humido folutum attra. hentes.

TACTVS: Papillae molliufculae, figuram imprefam breui affumentes.

His gaudent plurima Animalia, fed non omnia. Plura fi Crea. tori addere placuiffet, plura percepiffent; vti Magnete praefentiam Ferri, Eledtro electriciratis phaenomena. Antennas Infectis folis concelfit, nobis aeque ignotas ac illis Aures.

Indicat Oculus ex luce, Auris ex aethere appropinquantia; percipit Taffus ex vnione folida praelentia; examinat Nafus volatilia neruis, Lingua folubilia fibris, affumenda: f. conceffa falutaria, $f$. vecita noxia.

COGITANS allicit ad velle gratum, aut nolle ingratum.

GAVDIVM puerile, fanguineum, rubrum, oleofum, fpongiofum, tepidum, libere pulfans, anhelans, ridens, tranfpirans, promtum. Vita.

METVS iuuenilis, phlegmatic. pallefcens, aquofus, laxus, horrens, debile pulians, dyfpnoicus, fuffocans, cacans, tremens. Morbus.

IRA virilis, cholerica, fufca, fpirituofa, ftrikta, feruens, dure pulfans, afthmatica, ftertens, miluriens, agitans. Medicina.

MOEROR fenilis, melancholic. arer, acidus, rigidus, frigidus, tarde pulfans, orthopnoicus, fufpirans, obftipans, quiefcens. Mors.

Sic Gaudium bonis fruitur, Metus fuga eripit, Ira armis defendit, Moeror amiffa luget.

IMPERANTIVA cauffa quemadmodum Populi non funt nati, fed fubditorum ordini feruando Imperantes conftituti, ita Vegetabilium cauffa Animalia Phytiphaga, phytiphagorun Carniuora, et ex his Maiora ob parua, Homo (qua animal in oeconomia naturae) ob maxima et fingula, fefe vero praecipue, faeua mercede conducta tyrannidem exercent, vt Proportio cum nitore Reipublicae naturae perennet. Vicifiun finguli ciues confpirant in Maieftatem Hominis rationalis imperantis, cuius eft fummum Reipublicae auctorem agnofere. 


RES PVBLICA Naturae, tanquam Aqua e Fontibus in Rinulos, Amnes, Fluuios ad Mare tranans, e numerofifima Ple$b e$ in pauciores Nobiles, pauciffimosque Magnates ad Imperantem adicendit, dum Animalia minima, numero, vi, potentia facile infinita, in vfum cedant maioribus, inertioribus, praeftantioribus, cum natura nunquam magis quam in minimis tota fit.

OPERATIONES incolarum praecipuae funt: I. Multiplicare Speciem, vt negotiis fufficiant. 2. Auferre immunda, cadauera, languida, confpurcata, ftagnantia, acida, putrida, vt nitor aulae fulgeat. 3. Detondere quotannis vegetabilia, vt renouetur annulim theatrum; 4. Aequilibrinm inter Species Animalium et Vegetabilium feruare, vt proportio perennet. 5. Vindicare fe ipfos ab interitu, ne vacet adminiftratio.

MINISTRI, propriis muniis praefixi, tot funt, quot animalium Species, finguli ad officia proprio commodo allecti, quum ex labore fuftentationem fuam reportent; ne quidquam deficiat, vbi nihil fuperuacaneum. Ne auten alter alterius negotiis fefe immifceat, fimulque lucrum alteri praeripiat; fub poena capitali fancita $L e x$, ipfa fenfibus, Olfątus imprimis et Guftus, infcripta, ne transgreffores excufabiles euaderenr. Sic rapina rerum omnium eft, quam Armis, Fulcris, Munimentis, Halitu eludunt viuaciora, dum Languida fuccumbant, et Vegetiora in motu feftinent, vt opus naturae perenni flore rideat.

IMPVLSORES etiam praefixi ad munera promte peragenda: blanda Venus ad propagationem irritat; auara Fames ad futtentationem impellit; atrox Dolor ad conferuationem cogit; neque haec fine Numine.

DIVISIO Naturalis Animalium ab interna ftrußtura indicatnr:

COR biloculare, biauritum;
Sanguine calido, rubro. $\begin{cases}\text { viuiparis } & \text { Mammalibus. } \\ \text { ouiparis } & \text { Auibus. }\end{cases}$

$C O R$ vniloculare, vniauritum; $\{$ pulmone arbitrario Amplibiis.

Sanguine frigido, xubro. $\{$ branchiis externis Pifcibus.

$C O R$ vniloculare, inauritum; $\{$ antennatis Inferris.

Sanie frigida, albida. $\{$ tentaculatis Vermitus. 
I. MAMMALIA.

Cor biloculare, biauritum; Sanguine calido, rubro. (*)

Pulnones refpirantes reciproce.

Maxillae incumbentes, tectae.

Penis intrans viuiparas, lactantes.

Senjuis: Lingua, Nares, Tąus, Oculi, Aures.

Tegmenta: Pili, pauci indicis, paucifimi aquaticis.

Fulcra: Pedes quatuor, exceptis mere aquaticis, in quibus pedes pofteriores in caudae pinnam coaliti.

II. A VES.

Cor biloculare, biauritum; Sanguine calido, rubro.

Pulmones refpirantes reciproce.

Maxillae incumbentes, nudae, exfertae, edentulae.

Penis fubintrans absque fcroto ouiparas crufta calcaria.

Senfus: Lingua, Nares, Oculi, Aures absque auriculis.

Tegmenta: Pennae incumbentes, imbricatae.

Fulcra: Pedes duo. Alae duae.

\section{A MP HIB I A.}

Cor vniloculare, vniauritum; Sanguine frigido, rubro.

Pulmones fpirantes arbitrarie.

Maxillae incumbentes.

Penes bini. Oua plerisque membranacea.

Senfis : Lingua, Nares, Oculi, multis Aures.

Tegmenta coriacea nuda.

Fulcra varia variis, quibusdam nulla.

IV. PISCES.

Cor vniloculare, vniauritum; Sanguine frigido, rubro.

Branchiae extus comprimendae.

Maxillae incumbentes.

Penes nulli. Oua absque albumine.

Senfus: Lingua, Nares? Oculi (non Aures).

Tegmenta: Squamae imbricatae.

Fulcra: Pinnae natantes.

(*) In omnibus animalibus, quibus fanguis non calet, ventriculus cordis eft vnicus. Hall. Boerb. 1. p. 117,197 . miratus itaque quod 2 ventriculos cordis Crocodilo adfcribat Haffelquift iter 293. 

Quadrupedia. L.1935. (Wammalia L.1758)( nadru

Aves. 2.1735

Amphibia L. ras.

Pisces. 2. 1735 
Insecha L.1735. Pedes 6

Cundacea Brisosm 175t. Pedes 8 et iltra.

Rermes. Li:1735. 


\section{REGNVM ANIMALE.}

V. INSECTA.

Cor vniloculare, inauritum; Sanie frigida.

Spiracula: Pori laterales corporis.

Maxillae laterales.

Pentes incrantes.

Senfis: Lingua, Oculi, Antennae in capite absque cerebro.

(non Aures, Nares).

Tegmenta: cataphrakta cute offea fuftentante.

Fulcra: Pedes, quibusđam Alae.

\section{VERMES.}

Cor vniloculare, inauritum; Sanie frigida.

Spiracula nulla?

Maxillae multifariae, variae variis.

Penes varii Hermaphroditis Androgynis.

Scnfits: Tentacula, caput nullum (vix Oculi, non Aures, Nares).

Tegninenta interdum calcaria vel nulla, nifi Spinae.

Fulcra: nulli Pedes aut Pinnae.

Terribilia funt opera Tua DOMINE,

In multitudine virtutis Tuae, Te metientur inimici Tui. 


\section{CLASSISI.}

\section{A M M A L A.}

MAMMALIA baec et nulla alia mammata Animalia, ex ftructura, vifceribus, organis homini proxima, pleraque Q Ill- $^{-}$ drupedia, nobiscum Continentem inhabitant, tanquam inter hoftes atrociffimos, dum pauca $P$ innata intra Pulagum fe fubducunt.

VESTITVS Quadrupedium e Pilis fexilibus, mollifimis, diftinetis, iniuriae minus obnoxiis; iisque fertilioribus in frigidis quam aeftuantibus terris. Hi vero Pili in Aculeos coaliti (Erinaceo, Hyitricibus), aculei explanati in Squanias (Mani), fquamae coadunatae in Scutunn (Dafypis); at pilorum vellus faepe difinctum Suturis (Cani, Equo), quae elongatae in $I t$ bam (Equo, Sui); Sed Aquarica nuda euafere, ne marlefierent, nifi in terram exire aliquando neceffe habeant. Praeterea Facies faepe Verrucis, Labia Vibriffis, Mentum Barba (Homini, Simiae, Caprae) et Pedes Callis non raro notata funt.

FVLCRA, motus inftrumenta, pro expeditiori fuga in euitando hoftem, aut affequendo vraedam, TERRESTRIBVS funt Pedes IV, quorum anteriores $P$ almis inftuuntur, interdum tanquam Manus remoto pollice diuifae (Primatibus, Didelphibus); Pofteriores autem Plantis, vt validius properarent, et hi vel Palmati digitis membrana connexis, vt natarent, vel $F i f j i$ digitis feparxis, vt facilius currerent, aut Talis innixi, vt firmius perfifterent (Homini, Vrfo); Ne vero fuga detererentur inter afpera, vel denfo vellere manicati (Lepori timido, Vulpi Lagopodi, Bradypis), vel I'ngulis tanquam calceis induti funt digiti (bifulcis Pecoribus, Suibus), qui etiam fuccenturiatis muniti talis, vel Solidipedes indiuilis pedibus (Equis); fed in manibus faepe vngues plani, orbiculati (Homini, Simiis, Lemuribus); At vero rapaciores Ferae vnguibus armatae fubulatis vt praedam apprehenderent, lacerarent, terramque ofoderent; fed Volantia vel manibus elongatis palmatisque aëra fcandunt (Vefpertiliones), vel membrana inter palmas plantasque expandenda, velificant (Lemur, Sciurus, Mus). Aliter A Q va t ic A, quorum brachia intra latus retracta, et femora poftice coadunata plantis in caudam horizontalem (Cetis). 
Classis Quadrupedia. S.1935. (Hommalia 2.1758; (Anadrmpeda Briss. 1756.) 

ARMA Mammalium praeter dißtos vngues, nominandosque Dentes, imprimis Cormua cartilaginea capiti inferta funt; haec vel jólida perennia (Rhinoceroti), vel ramojá annua, primumque pilofa apiceque crefcentia (Ceruis), vel cauta vaginantia bafique creicentia (Bobus, Capris, Ouibus).

CIE AR I A inftrumenta Dentes audiunt, qui triplices: Primores faepe compretî incifores, pro euellendo, rodendo, confcindendo; Laniarii conici longiores pro lacerando; Molaves latiores pro conterendo, et hi obtufi phytiphagis, acutiotes carniuoris; at edentula folae Myrmecophagae et Manis euafere.

VMBRACVLV M eft Cauda, qua turpiffimae abfconduntur partes; haec paucis muila (Homini, Simiis et Muribus nonnullis), abbreniata nec femoribus longior (Lepori, Satyro, Talpae, Erinaceis), elongata ad pedum longirudinem et vltra (Canibus, Muribus, etc.); harum fingulae vel Nuddae (Muribus), vel Prebenfilis conuoluendae proque quinta manu inferuientes (Simiis, Hyftrici, Didelphibus), vel Comofae longis fetis (Equis, Bobus), vel Floccofae apice penicilliformi (Leoni mari, Muri laculo), vel Diftichae verinque pilofae (Sciuris, Myrmecophagae).

CVSTODES Animalium Senfus funt pro conferuatione indiuidui; fed rerreftribus Aures inftruetae Auviculis varia figura Iobatis, pro acutiore auditu; Aquaticis vero auriculse nullae; Nafius figura varia interdum elongatus in probojcidem (Elephanti, Beftiis), firus fupr? Labium fuperius fiffum (Gliribus) aut lase cunofun (plerisque).

GENIT A LIV M curiofior indagatio abominabilis difplicet, quama uis varia et fingularis Clitoride, Nymphis, Scroto, Pene, quae tamen poffet ad ordines naturales viam monftrare.

VENERE Vaga pruriunt pleraque, vbi Mares dimicant inter $\mathrm{fe}$, quo valentiores polleant pro fobole viuaciore, ineuntes feminas viuiparas foetum intra fe excludentes, quem enixum lactant, defendunt, curant in proximas nuptias, adultioremue aetatem. Polyganna aurem nonnullis, vbi Mas gynaeceum ex pluribus feminis libi affociatis defendit (Phocae); pauciffimis vero Monugamill eft, dum Mas er Feminı indiuidni focii curam gerunt foetus (Simiae nonnullae, Lemur, Vefpertiliones, Erinaceus). 
MAMMAE lactantes feminis omnibus, etiam Maribus (excepto Equo) numero determinatae: Pectorales (Primatibus, Cetis); Abdominales (Didelphibus, Phocis); Inguinaies (Pecoribus, Belluis); Abdominales Pectoralesque fimul (Gliribus pluribus); longitudinaliter digeftae (Suibus aliisque), at fiepius binae pro fingulo foetu ordinario.

colvntvr varia imprimis Fecora ob Carnes, Lac, Corium, Vellera, Pinguedinem; ad Onera vero Equus, Camelus, Elephas; inftituuntur Ferae variae pro venatu, muribus, ferpene tibus; Viuariis afferuantur rariora.

ORDINES imprimis a dentibus defumuntur:

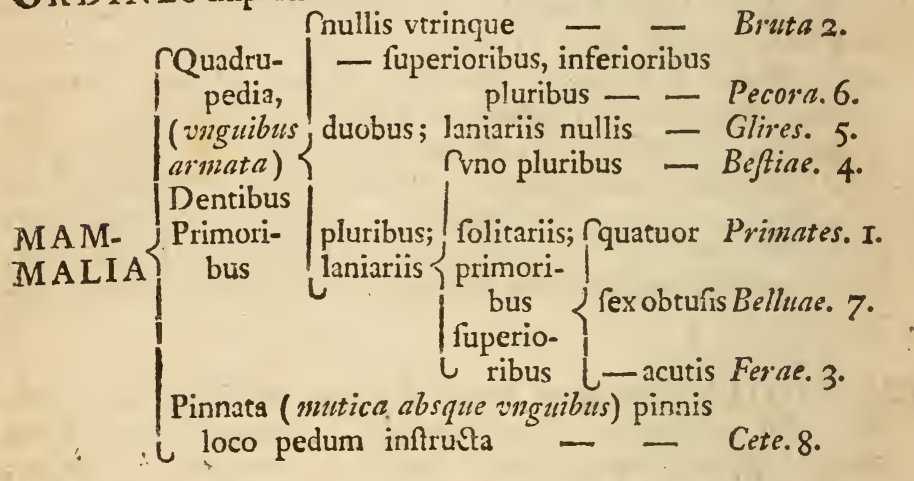

\section{PRIMATES.}

Dentes primores fuperiores IV paralleli. Laniarii folitarii. Mammae pectorales, binae.

Palmae Manus Junt.

Brachia diduct a clauiculis, inceffu tetrapodo valgo.

Scandunt arbores earumque gazas legunt.

\section{BRVTA.}

Dentes primores mulli fuperius aut inferius.

Inceffus ineptior.

III. FER A E.

Dentes primores vtrinque: fuperiores VI, omnes acutiores. Laniarii folitarii.

Vngues pedum acuti.

Victus ore faenientium s cadameribus, rapina. 
Ordines: 1. Anthropamorpha 2.1735 (Antropomorpha R. 1940.) (Primates 2.1950)

2. (Bruta. 2.1950.) Agride R.1748.

3. Ferae. L. 1735.

4. Bestiae. 2.1750.

5. Glires. A. M3s.

6. Pecora. L. M3s.

7. Jumenta. L.1y35. (Belluae L. m58.)

0. Plagiuri Ath. 1935. (Physeteres Klim.114) (Cete 2.1758) (Cetacea Briss.1756)

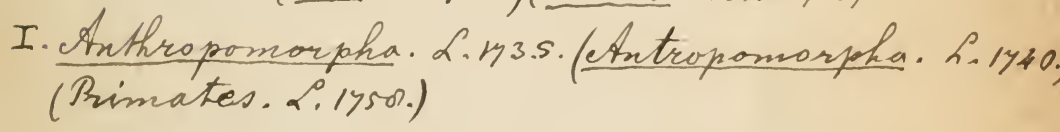

II. Mrunta. L. 1950.) Atgriae L.1948.

III. Ferae. C. 335 . 
IV. Bestiae. L.1750.

V. Glines. L. m3s.

VT. Pecora. R. 1435.

DII. Jumenta 2.1735. (Belluas Q.1750.)

1III. Pagivi int.1735. (Physeteres Klein 1741)(Cote. 2.1758.)

(Cetacea)Briss. 1756) 
IV. BESTIAE.

Dentes primores virinque mumero indeterminato. Lanisrii femper vno plures.

Nafus elongatus, fodiens.

Victus e fuccofis radicibus, vermibus.

\section{GLIRES.}

Dentes primores fuperius inferiusque 2. Laniarii $m u l l i$.

Pedes curfit falicntes.

Vißtus rodendo cortices, radices, vegetabilia, etc.

\section{PECORA.}

Dentes primores inferiores plures, fuperiores nulli.

Pedes vngulati, bifillci.

ViRtus cuellendo plantas muminandas.

Ventriculi 4: Ingluuies macerans, ruminans; Reticulum cancellatum, recipiens; Omafus multiplicatus, confunesus. Abomafus fafciatus, acefcens Coagulo pro febo, vt unimus do salefcant.

VII. BELLVAE.

Dentes primores pluves, obtuli.

Inceffus grauis.

ViCtus extrabendo vegetabilia.

VIII. CETE.

Pedum loco pinnae pectorales et caudae (plagiura) plana. Vr. gues mulli.

Dentes cartilaginei. Nafus front is faepe fiftula.

Victus e Mollinfcis, Pifcibus.

Locus pelagicus.

Hos a Pifcibus diuulfos iuff Mammalibus a fociari ob Cor biloculare calidum, Pulmones refpirantes, Aures cauas, Penen intrantem Feminam Mammis ladantem, idque ex lege natuvae iure meritoque.

AVCTORES: Gefuerus, Aldrouandus diffufe, epitomator eius Ionftonts, Raius, paucique alii paffim, fed mancam reliquerunt Hiftoriam, de nouo ordiendam fecundum notas proprias; Defcriptionibus attributorum, Diateta varia, Oeco- nomia propria, reliquis, vt folida grana e furfurum quisquiliis depurentur; exemplaria propofui in Cane domeftico, Ceruo Rhenone, mure indico, Oue Ariete. 


\section{GENERVM Characteres compendiofi.}

\section{PRIMATES.}

I. Номо nofce te ipfum.

2. S I m I A Dentes laniarii, binc remoti.

3. LEMVR Dentes primores inferiores 6.

4. VESPERTILIO Manus palmatae volatiles.

\section{BR V T A.}

5. ELEPHAS Dentes laniarii et molares; Nafus probofcide elongutus.

6. TRICHECVS Dentes laniarii fuperiores; molares ex offertgofo. Pedes poftici coadunati.

7. BRADYPVS Dentes laniarii et molares. Nafus obtufuls.

8. my

9. MANis Dentes nulli. Corpus Jquamatum.

\section{FERAE.}

10. HOCA Dentes primores inferiores 4. Auriculae mullae.

II. CA N IS Dentes primores fuperiores intermedii lobati.

12. FELIS Lingua aculeata. Vugues retractiles.

I3. VIVERRA Lingua aculeata. Vngues exferti.

I4. MVSTELA Dentes primores inferiores conferti: duobus alternis interioribus.

I5. VRSVS Dentes primores fuperiores excaunti. Penis offe flexuofo.

\section{BESTIAE.}

16. svs Dentes primores fuperiores 4, inferiores 8.

17. DASYPVS Corpus tefta zonis interfecta.

I8. ERINACEVS Dentes primores fuperiores 2 , inferiores 2.

I9. TALPA Dentes primores fiuperiores 6, inferiores 8.

20. SOREX Dentes primores fuperiores 2 , inferiores 4.

2I. DIDELPHIS Dentes primores fisperiores IO, inferiores 8 . 


$$
\text { TGlires L.173. }
$$

22 Rhinoceros $\mathcal{L} 1748$.

23 Fbystrix $\therefore 173.5$.

24 Lepus $\mathcal{L} 1735$

25 Castor 21735

26 16us L.1735 (Jorex Brios. 1756).)

26 a Glis Baiss 1756.

26 b Cumiculun Briss 1756. (Cavia Kleire 1751.)

27 Sciums L.173.5.

TI Pecoza L 173 s.

28 Camelus 21735 .

29 . 160schns 21748

30 Cerrus 21735.

30 a Cinafta Bulas. 1756 .

31 Capra 2.1735 (bircus Brios. 1756)

31 a Tragulus Briss. 1756

32 Ovis $\mathrm{S}_{1} 1735$ (Aries Brios.1756)

$33 \widehat{B 00} \cdot 1735$

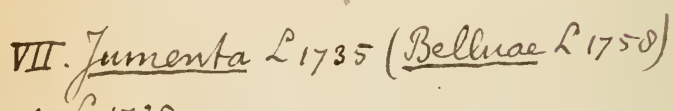

34 Equno 21735

35 Z6ippopotamme $2-1735$

35 a Tapione Brios. 1756. VIII. Plagiuri Art.1735 ( Phypetores Hlim 1741) (Cetacea

A. Fam.......... Art 1735: (Ceratodon Briss.1456)

B Fam. Balaenae Kcein 1741.

37 Balaena Ar2 1735

38 Physeter Ant 1938

38 a. Catodon At 1735 (Catus (Biss. 1756)

C. Fam. Delphoces Klein 174 ' (Porcelli Kcein 1741) 39 Delohimus Art 1735 . 


\section{GLIRES.}

22. RHINOCEROS COrmU nafale.

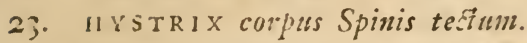

24. LEPVS Dentes primores fuperiores duplicati.

25. CASTOR Dentes primores fuperiores angulo recto excauati.

26. MVS Dinte. primure's fuperiores fubulati.

27. Scivers Dentes primures fiperiores cuncati; inferiores compresfis.

\section{PECORA.}

28. CAMELVS ecornis dentibus laniariis pluribus.

29. MOSCHVS ecornis dentibus laniariis folitariis: Juperioribus exfertis.

30. CERv' Cormua folida, ramofa, decidua.

3I. CAPRA Cormua tubulo fa erecta.

32. Ovis Cormua tubulofa reclinata.

33. во о Corkua tubuloja porrectu.

VII. BELLVAE.

34. EQYVS Dentes primores approximati.

35. H ргоротамvs Dentes primores diftantes.

VIII. CETE.

36. MONODON Dens in maxilla fuperiore exfertus.

37. BALAENA Dentes cornei in maxilla fuperiore.

38. PHYSETER Dentes in maxilla tantum inferiore.

39. DELPHINvS Dentes in vtraque maxilla. 


\section{P R I M A TES.}

\section{Dentes Primores fuperiores IV, paralleli. Momniae pectorales II.}

\section{r. HOMO nofce Te ipfum (*).}

Sapiens. I. H. diurnus; varians cultura, loco.

Ferus.

terrapus, mutus, hirfutus.

Iunenis Vifinus litbunnus. $\quad$ 166r.

Iuicenis Lupinus beffenfis. 1344 .

Inuenis Ouinus bibernus. Tulp. obf. IV: 9 .

Inuenis Hannoueranus.

Pueri 2 Pyrenaici. 1719.

Iobannes Leodicenfis.

Annerica - $\alpha_{\text {. }}$ rufus, cholericus, ręus.

nus. Pilis nigris, rectis, crafis; Naribus patulis; Facie ephelitica, Mento fubimberbi.

Pertinax, hilaris, liber.

$P$ ingit fe lineis daedaleis rubris.

Regitur Confuetudine.

(*) NOSSE SE IFS VM gradus eft primus fapientiae, diAtumque Solonis, quondam fcriptum litteris aureis fupra Diance Templum. Muf. ADOLPH. FRID Prafat.

Phyfzologice: Te contextum Neruis, intertextum Fibris, Machina tenella fed adolefcente in perfectiflimain, facultatibus inftructam fere omnibus, pluribusque, quam reliqua cunfa. Nudum in nuda bumo, natuli die, abiecit natura ad ragitus ftatim et ploratum, manibus pedibusque diuinciendum Animal caeteris imperaturum; cui fcive uibil fine doctrina: non fari, non ingredi, non vefi, non aliud natura spoute. Plin. Vides itaque qualem vitam nobis rerum natura promifit, quae primum nafientium omen fletum effe voluit. Sereca.

Diaetetice: De fanitate et tranquillitate, fi noneris, felicem: Moderatis conferuandum, Nimiis deftruendum, Variatis afficiendum, Infuet is frangendum, Confuetis indurandum; polyphagum Culina inftructifima, per errores gratiffina, igne vinoque horrenda. Parwo fames conftat, magno faftidium. Seneca.

Patbologice: To tumidam vfque dum crepueris bullam, piloque pendulam in puncto fugientis temporis. Nibil enim bomine inbecillius terra alit. Homer. Nulli vita fragilior; nulli tot Morbi, tot Curae, tot Pericula. Breue vninerfum vtique aeui tempus. Pars nequa morti fimilis exigitur; nec rсputantur Infantiae anni, qui fen $\int u$ carent; nec Senectae in poenam viuaces: bebefcrnt Senfus, torpent Membra, praemoriuntur Vifus, Auditus, Inceffus, Dentes, Ciborum inftrumenta.Plin. Sic magna pars mortis iam practeriit, quidquid aetatis retro eft, Mors tenet. Totum denique bunc, quem vides populum, quousque cogitas effe; cito natura reuocauit et condet; Mors omnes aegre vocat; iratis Diis propitisque moriendrm. Senec. II. 59 . 


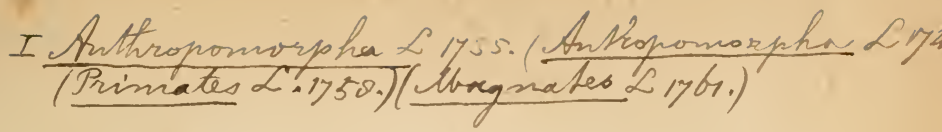

1. Homo L $y^{35}$. 1. sapiemo a 1758. 

Euro- $\quad \beta$. albus, fanguineus, torofus.

paeus. Pilis flauefentibus prolixis. Oculis caeruleis.

Leuis, acutifimus, inuentor.

Tegirur Veftimentis arctis.

Regitur Riribus.

Afiati- $\gamma$. luridus, melancholicus, rigidus.

cus. Pilis nigricantibus. Oculis fufcis.

Seuerus, fafuofus, auarus.

Tegitur Indumentis laxis.

Regitur Opinionibus.

Naturaliter: Te audacis naturae miraculum, Animalium Principem, cuius cauffa cuncta genuit natura, effe animal flens, ridens, melodum, loquens, docilc, iudicans, admirans, fapientifimum; fed fragile, nudum, fuapte natura inerme, ad omnem fortunac contumeliam proiectum, alienae opis indigens, anxiac mentis follicitaeque tutelac, precarii fpiritus, pertinacis fpei, qucrulae vitae, tardae fapientiae. Temporis annihilati emortui contemtorem, praefentis viuidi dilapidatorem, futmri dubii aeftimatorem in vita volucri, pretiofiffima, irreuncabili; fic optima quaeque dies miferis mortalibus aeui prima fugit; alium ad quotidianum opus laboriofa Egeftas vocat; aliuın Luxus incarcerat, altilemque fuffocat; alium Ambitio numquam quieta follicitat; alius Diuitiis, quas optauerat, metuit, et voto labo"at fuo; alium Solitudo, alium femper veftibulum obfidens turba. Hic fe habere dolet Liberos, hic fe perdidiffe; Lacrymae nobis deerunt, antequam cauffae dolendi. Sed quousque nos malos noftra mala rapuere! circumferre pericula, ruere in ignotos, iratos fine iniuria, ferarum more occidere quem non oderimus, fecundos optare ventos, quorum felicitas eft ad bella perferre; parum videlicet ad mortes noftras terra late patet. Sence. Ciastera animantia congregari contra diffimilia, at Homini plarima ex bomine mala. Plin.

Politice: Te reati loco tenere erroren publicum factum, qui te vix enixun confuetudinis larua induit, nutrit, educat, alit, regit, fecundum quam honeftus, fortis, fapiens, moratus, pius exiftimaris; Cum gubernatus Opinione viuas ad Confuetudincm, nec ad Kationem. Te, inter perituros conftitutum, cum nulli contigerit impune nafci, bencficii loco petere, vt vitimus ceruicem praebens, dum interim, dulci fortuna cbrius, colligendo in craftinum Lapillos fortunae certas, verbis in furorem ageris, aliter theoretice pios odio perfequeris, quantosque tumultus excitas, non ve feruias, fed cui; nugis irreuocabile rempus confumis; immortalia aeternaque animo volutas, de feris nepotibus pronepotibusque disponis, notras fpes, oblitus condition is Tuae, ponis, dum interea longa conantem mors opprinit, at in agone oculos ape-

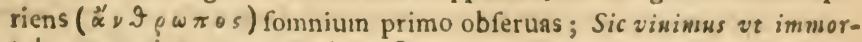
tales, et roorimar it mortales. Senec.

Moratiter: Te fub rudi larua Ineptum, Lafciuum, Imitatorem, Ambitiofum, Prodigum, Solicitum, Aftutuin, Aufterum, Inuidum, Auarum, transformari in Attentum, Caltum, Confideratum, Modeftum, Sobrium, 'Tranquillum, Sincerum, Mitem, Bencficum, fuis Contentum.Vvi aиimantıиж 
Afer. d. niger, phlegmaticus, laxus.

Pilis atris, contortuplicatis. Cute holofericea. Nafo fimo. Labiis tumidis. Feminis finus pudoris; Mannmae lactantes prolixae.

Vafer, fegnis, negligens.

$V$ ngit fe pingui.

Regitur Arbitrio.

Monftro- z. folo $(a)$ arte $(b, c)$.

fis. a. Alpini parui, agiles, timidi.

Patagonici maynin , fegnes.

b. Monorchides ve minus fertiles: Hottentotti.

Innceae puellae abdomine attenuato: Europaeae.

c. Macrocepbali capite conico: Chinenfes.

Plugiocephali capite antice compreffo: Canadenfes.

Habitat inter Tropicos Sponte gratisque; per reliquam.

Telluris totam continentem coacte.

CORPVS ercetum, nudum, Pilis raris remotifinis adfperfum, fubfexpedale.

C A P V T obouatum: Pilens pilofus : Syncipite obtufo, Vertice obtufifimo, Occipite gibbofo.

Facies nuda: Frons planiufcula, quadrata, Temporibus compreffa, angulis finu intra Pileum adfcendens. Supercilia prominula pilis extrorfum imbricatis, interftineta. Glabella planiufcula. Palpebra fiserior mobilis, inferior quieta; vtraque Ciliis exftantibus fubrecuruatis pectinata. Oculi rotundi : Pupilla orbiculata. Genae gibbae, molliufculae, coloratae. Malae compreffiufculae vti Buccae laxiores. Nafies prominens, labio breuior, compreffus, apice altior gibbofiorque: Naribus oualibus, intus

Luctus, Luxuria, Ambitio, Aurvitia, Viuendique cupido; curat atque etiam port se de futuro. Plin.

Theologice: Te vltimum finem creationis;

In Tellnris globum, omnipotentis magifterium, introductum; ratione

fapiente, fecunduin fenfus concludente, mundi contemplatorem; vt

ex opere agnofceres Creatorem omnipotentem, omnifcium, immenfum et fempiternum DEum.

(Caetera reuelata a Tbeologis explicanda.)

Duo namque funt quae in cognitionem DEI ducunt, Creatio et Scriptura. Auguftinus.

DEVS itaque Natura cognofcendus, dein Dostrina recognofcendus. Tertullian.

Homo folus DEVM contcmplatur, Naturae et Renelationis eundem AuẼorem: quem te Dlius effe

iufit, et bumara qua parte locatus es in re, difce, Perf. III. $7 t$. 


intus hirtis, margine craflufculo. Labium fuperius fubperpendiculare, Lacuna exaratum; Labium inferius erectiufculum, magis gibbum. Mentum prominens, obtufum, gibbofum. Aures larerales: Artriculac rotundato-lunatae, appreffiufculae, nudae, fupra margine fornicatae; infra gibbofae, molles.

T R V N C S conftans Collo, Thorace, Dorfo, Abdomine.

Collum teretiufcuium, capite breuius; Certucis Nucha concaua; Gula fupra concaua, medio fubtus gibba.

Thorax antice pofticeque depreffufculus. Peftus planiufculum, Iugulo excauato, Axillis concauis barbatis, Scrobiculo planiore. Mimnace duae, pectorales, diftantes, gibbae, rocundatae: $P$ (bpilla cylindrica, obtufa, rugofa.

Dorfum Tergo planiufculo, Humeris exftantibus, Interfcapulio depreflo.

Abdomen gibbum, laxum: Vmbilico excauato, Epigafrio planiore, Hypogaftrio gibbofiore, Hypochondriis rotundatis, Inguinibus planiufculo-concauis. Pubis regio barbata. Genitalia exferta: Pene cylindrico, Scroto fubrotundo, rugofo cute laxa, fittura longitudinali per Perinaeum excurrente; 1. Vulua gibba, compreffiufcula. Anus retractus.

ARTVS ex Manibus loco pedum anteriorum et Pedibus pofterioribus.

Manuum Armi diuaricati, craffiufculi, teretes, longitudine pedum. Cubitus obtufo-prominulus. Lacertae craffitie armorum, teretes, introrfum planiores. Palmae rotundatae, dilatatae, planae, extus conuexae; Vola concaua: Digiti V: Pollex remotus, breuior, craffior. Digiti 2, 3, 4, 5 approximati, horum 5 minor reliquis, dein 2, 4, fed 3 paulo longior. Vngues omnibus rotundati, pla. no-conuexi, lamula pallida.

Pedum Femora approximata, Lambis mufculofis, Natibus gibbis, carnofis, Genubus antrorfum verfis, obtufiffimis, fubrus concauis. Crura longitudine femorum, poftice mufculolo-ventricofa, inferne anguftiora, antice macra. Calcanei Talis infiltentes, prominuli, gibbi: Malleolis lateralibus, vtrinque oppofitis, hemifphaericis, duriufculis. Plantae oblongae, fupra conuexiufculae, fubtus planse, transuerfe concauae. Digiti V: omnes incurui, fubtus gibbi, approximati. Pollex crafior, breuior; 2,3 fubaequales, 4,5 decrefcentes; vltimo minimo. Vngues ot in Manibus omnes. 
Differt itaque a reliquis Corpore erecto nudo, at pilofo Capite, Superciliis, Ciliisque, tandem Pube, Axiilis, Maribusque Mento. Feminis Nymphae et Clitoris; Mammae 2 pectorales. Caput Cerebro omnium maximo; Vuula ; Facies abdomini parallela, nuda; Nafo prominente, compreffo, breuiore; Mento prominente. Cauda nulla. Pedes Talis incedentes.

Tróglodytes.
2. H. nocturnus $\left(^{*}\right)$.

Homo fylueftris Orang Outang. Bont. iau. 84. t.84. Kakurlacko. Kiôp. itin. c. 86. Dalin. orat. 5 . Habitat in Aethiopiae conterminis (Plin.) in Iauae, Amboinae, Ternateae Speluncis.

Corpus album, incefu erectum, noftro dimidio minus. Pili albi, contortuplicati. Oculi orbiculati: iride pupillaque awrea. Palpebrae antice incumbentes cum MemHrana nictitinte. Vifus lateralis, nocturnus. Actas $X X V$. annorum. Die caecutit, latet; Noßtu videt, sxit, furatur. Loquitur fibilo. Cogitat, credit Jii caufsa factam tellurem, fe aliquando iterann fore innpaciantem, fi fides peregrinatoribus.

(*) Gerus Troglodytac ab Homine diftinßtum, adhibita quamuis omni attentione, obtinere non potui, nifi affumerem notam lubricam, in aliis generibus non conftankem. Nec Dentes laniarii, minime a reliquis remoti; nec Nymphae Caffrac, quibus carent Simiae, hunc ad fimias reducere admittebant. Inquirant autoptae in viuo, qua ratione, modo notae aliquae exiftant, ab hominis genere feparari queat, nam inter fimins verfantem oportet effe fimiam. Apollodor.

Speciem Troglodytae ab Homine fapiente diftindiffimam, nec noftri generis illam nec fanguinis effe, ftatura quamuis fimillimam, dubium non eft: ne itaque varietatem credas, quam vel fola Membrana nictitans abfolute negat.

Afri Pilos contortuplicatos, quamuis albos, in hoc miratus fum, collatis imprimis Varietarum cauffis in Plantis, in Pullo Gallinaceo, nec tamen quidquam de Mauris albis ex nigris ftatui.

Praeadamitas nec dixi hos Troglodytas Plinii, quamuis nos vltimum fimus Creationis opus.

Homo caudaius birfutus. Maupert. epift. 7. Kiôp. it. 79. Bont. iau. 85. an Aldr. digit. $=49$ ? incola orbis antardici, nobis ignotus, idenque vtrum ad Hominis aut Simiae genus pertineat, non determino. Mirum quod ignem excitet, carnesque affer, quamuis et crudas voret ceftimonio Peregrinantium. 
2 Troglody tes L Mso. ( Thomines webedive infecta? ?) 
2. SIMIA. Dentes Primores IV, approximati.

Laniarii folitarii, lorigiores hine remoti. Molares obtufi.

* Cauda nulla: Simiae veterum.

Satyrus. I. S. ecaudata fubdus nuda. Syft. nat.VI. p. 3 . Satyrus indicus. Tulp. obf. KII. c. 56 .

Habitat in Afrien (Afia.)

Magnitudo puevi fexennis. Dorfum crinibus nigris bir. futum, fubtus f. antice vondique glaber.

Sifframes.

Sylua- 2. S. ecaudata, clunibus tuberofo-callofis. Syft. nat. 3.

nus. Simia. Gefir. quadr. 847 .

Simius cynocephalus alter. Alp. aegypt. 241. t. 16.

Cercopithecus. Ionft. quadr. t. 59. f. 5 .

Habitat in Africa. Zeylona.

Frons ad fipercilionun regionem transuerfe gibba.Pellis vrfina. Scrotum tanzquan intra vuluan reconditum; vel penis intra Scroti foramen; auttunno Tefticuli intumefcunt. Sedes Feninae rapi inftar extuberans. Minatur minis, cacbinno blanditur, caffiortum more falutut. Sorbilia manu baurit. Femina menffruat.

Anatome. E. N. C. d.2. a.7.obf. 40.

** Cauda abbrcuiata: Papiones.

Sphinx. 3. S. femicaudata, ore vibriffato, vnguibus acuminatis. Syft. nat. 3.

Papio f. Baboon. Gefin. quadr. 352,t. 353. Allir digit. 260. 1onjt. quadr. 145. t. 6r. f. I. Rai. quadr. 158. Ilabitat in Borneo.

Magnitudo Molo/fi ; incedens quatuor pedibus; Scrotum pendulum; Animal libidinojum, feminis facile vim infert; litteras transferre addifcit.

Apedia. 4. S. femicaudata, palmarum pollice approximato, vnguibus oblongis: pollicum omnium rotundatis.

Simia cauda abrupta, vnguibus compreffis: pollice palmarum digitis adhaerente. Amocn. acad. 1. p. 27 S.

Habi-

Simia quam fimilis tupilfuma beftia nobis! Ennius : quod Simiae Mammae, Vuula, Vterus, Cilia, Mufculi Loquelac, Vngues hominis funt; non vero Rete inirabile, Clitoris, Nymphas. 
Habitat in Indiis.

Magnitudo et colsr Scinri fubgrifei; fimilis S. fciureae. Pollex manumn anicarum non renuotus. Vngues onnes oblongi, compreffi, fed pollicum tam anteriorum, quan pofticortun busnanis fumiles. Cauda brenifinna, vix pollicis trazsuerji.

*** Cauda elongata: Cercopitheci.

Silenus. 5. S. caudata barbata, corpore nigro, barba niuea prolixa. Simia Callitriches magnitudine cynocephalorum. Alp. aegypt. 242 ?

Habitat in Aira: Zcylona, Inta etc..

Species obfurior, ignotis Pedun vnguibus aliisque plurimis attributis.

Faunus. 6. S. caudata barbata, cauda apice fioceofa. Sy/t. nat. 3.

Cercopithecus barharus I. Cht:: exot. 37I. t. 37I. Nieremb. nat. 179. Rai. quadr. 153 .

Habitat.

Corpus nigricans; Darba, Pectus, anteriora abdoninis alba.

Panifcus. 7. S. caudata barbata, cauda prehenfili, palmis fubtetradacty!is. Syft. nat. 3 .

Simia fulca maior, palmis tetradactylis, cauda prehenfili ad apicem fubtus nuda. Brown. iam. 489.

Guariba. Maregr. braf: 229. t. 226. Rai. quadr. 153. Habitat in America meridionali: Brafilia.

Corpus nigrum. Pedes et Cauda dimidia exterior brannea; Caudae extremitas binc nuda, qua, quidquid $e$ terra cleuet, prebendit. Digiti Pedum-4-5. Z. Hollman; eft vero Pollex reliquis minur, introrfin a caeteris remotus, Vnguesque manuım rotundati, pedum fubulati. D. Aymen. Pullos in dorfo gerit, qui ambiunt matris corpus pofterius anguftius. Oriente et Occidente jöle congregintur concionem celebrantes: Marcgr.

Diana. 8. S. caudata barbata, fronte barbaque faftigiata. Linn. act. Stockb. $1754 \cdot$ p. 210 . t. 6.

Cercopithecus barbatus guineenfis. Marcgr. braf. 227. t. 228. Rai. quadr. 159.

Cercopithecus barbatus 2. Cluf. exot. 3II. Inbitat in Guinea. 


Magnitudo Felis maioris, nigra punct is albidis. Dorfum poflice ferrugineum. Femora fubtus belunla. Gula pectusque alba. Frons pilis erectis, albis, faftigriatis, linea transuerfa in forman lanae crejcentis. Barba faftigiata, nigra fubtus alba, infidens tuberi adipofo. Linea alba ab ano ad genua ab exieriori latere femorum ducfa. Ludibunida omnia deicicit, peregrinis motitando falutat; ircita ore biat maxillasque exagitat; vocata rejpondet greck.

Cephus. 9. S. caudata, buccis barbatis, vertice flauefcente, pedibus nigris, cauda apice ferruginea.

Cercopithecus barbatus alius guineenfis. Marcgr. braf. 228. Rai. quadr. 156 .

Habitat in America.

Corpus Jibbfuficum, fubtus fubcaerulefcens; Os caerule. fiens: barba fiauefiens.

Aygula. 10. S. caudata fubbarbata grifea, eminentia pilofa verticis longirudinali. $0 / \%$. iter. 99.

Habitat in India. Ojbeck.

Corpus grifeum lupi coloris, fubtus gula, pectore, abdomineque albicans. Cuuda corpore longior, cinerea, attenuata. Facies planiu/cula, albida, muda. Nafus depreljiss, breul'limis, ab o"ce reanotus, Incibna labii jiperioris gemina. Bucca fibbarbatn, longitudine menti jubbarbuti. Supercilia frontis gibbu, promincntin. Pedes nigri: Vngues pollicum rotumdati; reliqui oblongi. Auric lae acutiufiuilae.

Hama- II. S. caudata imberbis, auribus comofis. Syft. nat. 3 . dryas.

Jachoes

Iacchus. 12. S. caudata, auribus villofis patulis, cauda hirfutifima longifima, vnguibus fubulatis: pollicum rotundatis.

Simia auribus villolis patulis, vnguibus pollicum fubrotundis. Sy/t. nat. 3 .

Cercopithecus brafilianns 3. Sagouin. Clus. exot. 372. t. 372. Rai. quadי154.160.

Cagni minor. Martgi.braf. 227. Pet.gnz.26.t.f.5. Klein dub. Ir.t. I.

Habitut in America. 
Cauda denfo vellere anmulata. Aures villofi, extus albi. Superba, iniuriarum impatiens, altiffime clamitat, delicatula.

Oedipus. 13. S. caudata imberbis, capillo dependente.

Cercopithecus minimus mexicanus, capillitio niueo. Edw. an. 195. t. 195 .

Habitat in America.

Corpus paruum, fibtus album. Capillitium dependens, bunano finile. Cauda longa, nigra, introrfun auruntia.

Aethiops. 14. S. caudata imberbis, vertice pilis arre?tis lunulaque frontis albis.

Simia aethiops. Hajfelqus. iter. Igo.

Habitat in Aethiopia. Haffelquijt.

Corpus fratura Dianue, fujcum, fubtus albunz. Cauda tecta, Jubtus ferruginea. Supercilia f. lunula alba transuerfa. Palpebra Jiperior nuda, alba. Aures acutiufculae.

Midas. 15. S. candata imberbis, labio fuperiore fiffo, auribus quadratis nudis.

Cercopithecus niger minimus, manibus et pedibus croceis. Edw. au. 196.t. Ig6.

Cay. Rai. quadr. 155 .

Hubitat in America.

Manus et Pedes lutei aut rubri. Corpus atrum. Cauda longiffina. Aures mudae, amplae, retufae. Vingues ommes fubulati $f$ ferini, exceptis pollicum plantarum rotundatis $\int$. bumanis.

Cyna- I6. S. caudata imberbis, naribus bifidis elatis, clunibus molgos. $:$ tuberofis. Syft. nat. 3 .

Simia aegyptiaca cauda elongata, clunibus tuberofis nudis. Haffelqu. iter. I89.

Cercopithecus angolenfis maior. Marcgr. braj:227. Rai. quadr. 155 .

Habitat in Africa.

Color lupi ex fiufco viridi-flauefcens. Statura vr/i. Nates caluae. Cauda arcuata. Abdomen albicans. Noctu vigilias inftituit.

Apella. I7. S. caudata imberbis, cauda fubprehenfili, corpore fufco, pedibus nigris. Muf. ad Fr. I. t. I. 

3. Irssimia Briss. ryt. (Lenuer L 1yso.-) 
Habitat in America.

$V$ ndique circumpiciens, Sono pulli Meleagridis famelici

Morta. I8. S. caudata imberbis, ore fufco, corpore fpadiceo, cauda nuda fubfquamola.

Cercopichecus americanus minor Monkie diftus. Seb. muff: I. p. 52.t. 33. f. I.

Hubitat in America.

Capuci- I9. S. caudata imberbis, cauda longa hirfuta, facie flauena. fcente. Muf. Ad. Fr. 2. t. 2 .

Habitat ....

Clanore borrendo boftes pellit; ftridet faepius viti Cicada; irata latrat more catuli.

Sciurea. 20. S. caudata imberbis, nccipite prominulo, vnguibus qua* tuor plantarum oblongis. Mil: Ad. Fr. I. p. 3 .

Habitat in India.

Corpus Sciuri, grifeo-virens, fubtus pallidum. Vlnae es Tibiae ferrugineae. Cauda villofa, corpore duplo longior. Pollicum vigues rotundati.

Syrichta. 2I. S. caudata imberbis, ore ciliisque vibriffatis. Syft.nat.3. Cercopithecus luzonicus minimus. Pet. gaz.21.t.13.t.1I. Habitat in Luzonum infälis.

3. LEMVR. Dentes Primores fuperiores IV : intermediis remotis.

Inferiores VI, longiores, porrecti, compreffi, paralleli, approximati. Laniavii folitarii, approximati. Molares plures, fublobati: antici longiores acutiores.

tardigra- I. L. ecaudatus. Muf. ad Fr: I. p. 3 .

dus. Simia ecaudata, vnguibus indicis fubulatis. Syft.nat.5.n. 2.

Ani-

Species Simiarsm inter Mammalia plurimae, fad panciffumae vire defcriptace et cognitae, plurimae obfcurae ignorantur.

Simiac sujpectse, memores, petulantes, imitatores, gefficulatores, indociles, minantur, vident, cilos bucca macerant, pediculos venantur, eads excellsut, notos vlnis gerunt, menftrant, terrefactae cacant immundae, vrinae ments farpe diftindto a :ulua fingulares. 
Animal cynocephalum tardigradum. Seb. mus: I. p. 55 . t. 35 f. I. 2. et t. $47 . f$. I.

Animal elegantiffimum robinfoni. Rai. quadr. I6I. Habitat in Zeylona.

Statura Sciuri, Jubferruginea, linea dorfali fubfufca; Gula albidiore. Linea longitudinalis oculis interiecta alba. Facies tecta. Auriculae vrcbolatac, intus bifoliatae. Pedum palmae plantaeque nudae. Vngues rotundati: indicum plantarum vero fubulati. Cauda fere nulla. Mammae 2 in pectore; 2 in abdomine verfius pectus.

Animal tardigradum, auditu excellens, monogamnım.

Catta, 2. L. cauda annulata.

Simia-Sciurus madagafcarienfis, fiue Maucauco. Edwo. au. 199. $t$. I99.

Habitat in Madagafcar.

Statura Felis, fiubcinerea. Oculorum regiones nigrae. Caput acuminatum. Aures tectae. Cauda longa, picta annulis atris cinereisque. Vngues fibulati: pollicibus plantarum rotundiores; fcandit wt Simia, fed tardiufcule. Habitu accedit ad Genettam auctoruin?.

volans. 3. L. caudatus, membrana ambiente volitans. $f$

Cato-Simius volans camelli. Pet. gaz. I4.t. 9. $f$. 8 . ACt. angl. 277. 12. 1065.

Vefpertilio admirabilis. Bont. iau. 68. t. 69.

Felis volans ternatea. Seb. mul. I. p.93. t. 58. f. 2. 3 . Habitat in Afia.

Membrana more Sciuri volantis $\int$. Muris volantis a capite ad manus, a manibus per latera ad plantas, a plantis ad apicem candue expanja. Vngues acuti. Mammae duae pectorales. Edit fructus arborum. Ex. suannis videtur Lemunibus f. Simiis proximum Animalculum, fed nobis non vifunn; vlterius exanninent autoptae. 

4 Tespentilis a.1735. (Pteropuro Brios.nyst. 
4. VESPERTILIO, Dentes erecti, acuti, approximati: primores 4 aequales. Manus palmatae, volitantes membrana corpus cingente.

Vampy= I. V. ecaudatus, nafo fimplici, membrana inter femora IUS. diuifa.

Vefpertilio cauda nulla. Syft. nat. 7 .

Canis volans ternatanus orientalis. Seb. muf. r. p. gr. t. $57 . f .1 .2$.

Vefpercilio ingens. Cluf. exot. 94. Edwa. au. I80.t.180. f. 2. 4 .

Halbitat in Afia.

Noifu baurit fanguinem dormientium, criftas Gallorum et laciymas palmarunz.

Spe- $\quad 2$. V. ecaudatus, nafo infundibuliformi lanceolato.

Etrum. Canis volans maxima surita. Seb. mus: 1. p. y2. t. 58. $f$. I.

Habitat in America aufrali. Rolander.

Nares extus infiundibulum referentes, fuperne adfcendentes in folium lanceolatum. Aures oututae, interius lacinia fibulata membranacea, longitudine Auriculae. Dentes Prinnores 4. Laniarii Jolitarii, magni, approxinnati reliquis. Molares anteriores breuiores, obtufiores. Alarum digiti 4, quorum I. annexus 2do; Pollex breuis vngue arcuato. Pedes digitis 5 aequalibus, vnguibus arcuatis. Calcaneus exferit tendinem fubulatum fecundum marginem membranae inter pedes pofticos, non coeuntem cun oppufito. Cauda nulla.

perfpicil- 3. V. ecaudatus, nafo foliato plano acuminato. Syft. nat. latus.

7. Mus. Ad. Fr. I. p. 7 .

Vefpertilio americanus vulgaris. Seb. muf. I. p. 90.

t. 55.f. 2. Edwo. Itr. 20I. t. 20I. f. I.

Vefpertilio roftro appendice auriculae forma donato. Sloan. iam. 2. p. 330.

Habitat in America.

An baec femina fequentis?

$\mathrm{C}_{2}$

4. V.

$V e \int p e r t i l i o r w m$ genus notturnum volitat Palmis expanfis palmaris membra. naque Laterali; Phalaenis inprimis vititans. 
Spafma. 4. V. caudatus, nafo foliato obcordato. Syft. nat. 7 . Glis volans ternatanus. Seb. mul: 1. p. 90.t. 56 .f. 1. Habitat in Afia.

leporinus. 5. V. caudatus, labio fuperiore bifido. Syft.nat.7. *Muf. Ad. Fr. . . p. . . *

Vefpertilio, cato fimilis, americanus. Seb. muf. I. p.89. t. $55 . f . \mathrm{I}$.

Habitat in America.

auritus. 6. V. caudatus, nafo oreque fimplici, auriculis duplicatis capite maioribus.

Vefpertilio auribus maioribus. Frifcb. all.t. 103. Edw. au. t. 201.f. 3. Olear. mul: t.15. f. 1.

Habitat in Europa.

Diffincta jpecies auriculis, nifi folo fexu a fequenti aliena?

murinus. $7 . \mathrm{V}$. caudatus, nafo oreque fimplici, auriculis capite minoribus. Faun. fuec. 18. Syft. nat.7.

Vefpertilio. Bell. aul. 147. Gefin. au. 694. Aldr. ornith. I. p. 57 r. Ionft. aul. 53. t. 20. Alb. au. 3. p. 95.

t. 101. Rai. quadr. 243. Edw. au. t. 201 . f.2.

Vefpertilio auribus minoribus. Frifcls. au.t. 102.

Habitat in Europa.

Noctu volat, victitat imprimis Pbalaenis, capitur Aräii Lappae calycibus, bybernat, de terra non euolat; icnenatus.

Anatome. E. N. C. d.2. a. I. obf: 48 .

II. B R V- 

II. Agriar 2.1948. (Brutân 20.1950.)

5. Elephas L.173s. 


\section{B R V T A.}

Dentes Primores mulli atrinque.

5. ELEPHAS. Dentes Primores nulli.

Laniavii fupériores elongari. Probofcis longiffima, prehenfilis. Corpus nudiufculum.

maximus. I. EL EPHAS. Rai. quadr. 123. Syft. nat. II. Seb. mut. I. t. III. $f$. I.

Elephantus. Gefr. quadr. 377. Aldr. quadr. 1. 1. c.9. Ionft. quadr. 30. t.78.f. 9 .

Habitat in Zeylonae paludojis ad amnes, edit Ramos, Cocos, Guilandinue fenina, Frumentum.

Maximum quadrupes. Oculi parki.Dentes Laniarii fuperiores exferti (Ebur). Aures ampliffrinae, pendulae, dentatae; act. angl. 277. p. 1051. Cutis craffiflima, callofa. Mammae 2. iuxta peifus. Vngues in apicibus loborum pedun. Genua flexilia. Collum breue.

Probofcis longiffma, extenfilis, acute odorans, loco manus ipfi inferuiens; ea cibum potunnque baurit, boflemque pellit; ea pracifa occiditur; murem metuit fub jomno ob tracbeac infirtionem. Retro coit et mingit. Portat domus, rectore collo infidente; in bello armatur falcibus; vulmefulo inter axin et atlanten furiojis occiditur; caeterum prudens, docilis.

\section{3 . TRI-}

Paxwax, Liganentкm albru, ad Caput elexardum ct Collkm Suftinendum, commune eft quadrupedibus; at deeft Homini et Simiis, cнm eredil plerumque Sedeant et incedant, nec eo indigsant. Raius. 
6. TRICHECVS. Dentes-Primores nulli.

Laniavii fuperiores folitarii.

Molares cx offe rugofo vtrinque inferius duo.

Labia geminata.

Pides pofteriores coadunati in pinnam.

Manatus. I. TRICHECVS. Art. gen. 79. Jyn. 107. Syft. nat. 37. Manatus. Rond. pifc. 490. Gejn. pijc. 213. Hern. m2xx. 323.

Manari. Cluf. exot. I33. Aldr. pifc. 728 .

Manati f. Vacca matina. Rai. quadr. I93.

Habitat in Mari Americano.

Labium fuperius muricatum Vibriffis rigidis, incuruis.

Pili corporis remoti, breutes. Pedes antici abbretiati, palmati, vnguiculati; poftici coadunati. Mammae 2. pectorales.

Edit vegetabilia; manfuefcit; muljica delectatur. Caro efculenta.

7. BRADYPVS. Dentés Laniarii obtufufculi remo. tiffimi.

Molares vtrinque obtuft:

Corpns pilis tectum.

tridacty- I. B. manibus tridactylis, cauda breui. Syf. nat. 6. p. 3 . lus. Amoen. acad. r. p. 487." Muf: Ad. Fr.4. ArEtopithaecus. Gefn. guadr. 869. t. 870. Ai f. Ignauus. Marcgr. braf: 22I. Cluf. exot. Iro. $t$. 373. Seb. mul. 1. p. 53. t. 33. f: 2. Olear. muf. 7 . t 6.f. 2. Pif.braf: 32 r.

Habitat in Americae mevidionalis arboribus; Victitat foliis teneris; non bibit; imbres metnit.

Gula flana.- Auriculae nullae. Cauda fübouata. Digiti combinati. Vngues palmarunn 3; plantarum 3. Mammae \& pectorales. Corpus valde pilofum. Vit a tenax. 


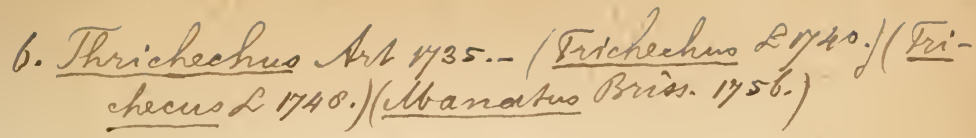

\%. Bradyfno L 1935. (Tardigradus Brin. 1956.) 
Dolbernecophaga. L.1540. 
Facile foandit, tardiffme et vix incedit, attonitus quafi caput flectit; fonns fenarius adjcendens; clamor borrendus, lacrymae miferae.

didacty- 2. B. manibus didactylis, cauda nulla. Syf. nat. 3. Muf. lus. $\quad A d$. Fr. 4 .

Tardigradus ceilanicus. Seb. minf. I. p. 54. t. 34. $f$. I. et $t$. $33 . f .4$.

Habitat in Zeylona.

Corpus pilis ferrugineis sndulatis. Caput rotundatum. Auriculae magnae. Vngues in palmis 2 in plantis 3 . Mammae 2 pectorales.

8. MYR MECOPHA GA. Dentes nulli.

Lingua teres, "extenfilis. Corpus pilis tectum.

didątyla. I. M. palnis didaßylis, plantis tetradakylis. Syft. nat.8. Muf. Ad. Fr. 8. *

Tamandua f. Coati americana alba. Seb. minf. I. p.60. t. $37 . f .3$.

Habitat in America auftrali.

tridacty- 2. M. palmis tridactylis, plantis pentadactylis. Syft. nat. \&. 1.s. Tamandua-guaçu. Marcgr. braf:"225. Pif. braj: 320. Seb. mul. I. p.60.t. 37.f. 2. et t. 40. f. I. Rai. quadr.24I. Habitat in America meridionali.

Vititat Formicis, infectis, quorum nidos vngue fecat. Macula nigra a pectore verfius latus ducta. Mammae Pectorales 2, Abdominales 6; lente currit, cauda lata inftar mufcarii je tegit, arborcs etiam fcandit.

tetrada- 3. M. palmis tetradactylis, plantis pentadactylis. Syft. Etyla. $\quad$ nat. 8 .

Tamandua I. Marcgr. lraf. 226. Rai. quadi. 242. Seb. mut. $27 \cdot t \cdot 4 \cdot f \cdot 2$.

Habitat in America meridionali.

Noctu exit; die dormit capite fub bracbiis recondito; irata baculum prebendit, ftertit, pofterioribus pedibus infidens. Candae extremitas calua, qua $f e$ ex ramis grborum fufjendere poteft, quod non in praecedenti.
C. 4
9. MA. 
9. MANIS. Dentes nulli.

Lingua teres, extenfilis.
Corpus fquamis tectum.

pentada- I. M. pedibus pentadactylis. Syft. nat. 8. Ait. Stockh. 1749 . etyla. p. $265 . t .6 . f .3$.

Armadillus fquamatus maior ceylanicus. Seb. mus. I. p. 88. t. 54. f. I. et t. 53.f. 5. Aldr. quadr. 667 .

Lacertus fquamofus. Clut. exot. 374: Bont. iau. 60. Olear. muf. 8. t. 7.f. I. Pet. gaz. 32. t. 20. f. x. 2. Rai. quadr. 274 .

Myrmecophagus fquamatus f. Daemon thebaicus. Herm. mulf: 295 .

Habitat in India orientali. Viętat Formicis, Vermibus, Lacertis.

Corpus fupra Squamis bafz friatis fetisque interftinetis veffitun vt ftrobilus; fiubtus birtum eft. Sed Cauda vtrinque Squamofa, etian fubtus inbricata.

Auriculae nudae rotundatae. Vngues primores medii maiores, quibus contractis incedit.

Myrmecophagis affinis. Irata fquanas erigit borrida.

Cauda pinguis in epulis expetita. 
9. Nbanis S. 1948. (Oholidotuo Briss. 1956.) 
III Araen. 2.1735

10. Phoce 2. 1y35. 


\section{F E R A E.}

Dentes Primores fuperiores fex, acutiufculi. Laniarii Solitarii.

10. PHOCA. Dentes Primores fuperiores VI paralleli: exteriores maicres. Inferiores IV, paralleli, diftincti, aequales, obtufiufculi.

Laniarii I: fuperiores ab inciforibus, inferiores a molaribus remoti. Molares V f. VI, tricurpidati. Auriculae nullae. Pedes poftici coaliti.

Uriena vrfina.

I. P. capite auriculato.

Vrfus marinus. ACF. petropol. 2. ann. 1749.

Habitut in Camfchatcae maritimis inter Afiam et Americam proximam, primario in infilla beringii.

Impetuofiffine natant, mares polygami gregatim degunt cum vxoribus, filiis, filiabus ad 120 ; coeunt in litore refiupinata femina, vix metuunt bominem, mordent proiectum lapidem, fenes clumbes quiefcunt pingues, proprium lapiden pro leço babênt, quen non dejérunt; bclla inftituunt propter vxores, leifum, aequum et iuffun; ficcumbente vno accurrit alter opem laturus, mox alteri alius, ne duo contra inum, ysque dum omnes praclio inuoluti: moefti lacrymas findunt copiofiffinas. Steller.

leonina. 2. P. capite antice criftato.

Leo marinus. Anfon. itin. 100. t. 100.

Habitat ad polum Antareticum.

C 5

Mas

Phocae genus natat fub aqua, vix incedit ob pedes anticos retraflos, popicos vnitos: victitat pifcibus; pingue. 
Mas labio fuperiore criftato. Dentes 2 fere exferti in maxilla inferiore.

Natant gregatim, pugnant pro feminis, excubias agunt.

Rofma- 3. P. dentibus laniariis fuperiozibus exfertis. Syft. nat. 6. rus. n. S.

Rofmarus. Olear. mur. 38. t. 23. f. 3. Ionft. pifc. t. 44. Worm. mutf. 289. Bonan. mue. 269. f: 27.

Mors f. Morfz, Rolmarus. Gefin. aqu. 2II.

Wall-Roff. Martens. fpitsb. 78. t. I. P. f. B.

Equus marinus f. Hippopotamus falfo dictus. Rai. quadr. I9I.

Habitat in̨tra Zonam arçicam Europae, Afrae, Americae.

vitulina. 4. P. dentibus laniariis tectis. Sy/f. nat. 6. n. r.

Phoca f. Vitulus marinus. Gefr. aqu. 702. Aldr. pifc. 722. Ionft. pifc. 44. Dodart. act. IgI. t. 19r. Rai. quidr. 189 .

Habitat in mari Europaeo.

Dormiunt in lapide ex aqua eminente, pariunt in glacie, per quam adfcendunt balitu, non defcendunt. Mammas retrabunt duas abdominis; inter Halecum cateruos Jaginantur, a Laris prouocatae ex/pituint pifces.

Anatome. E. N. C. d. I. a. 9. ob 9 . 98. et d.3. a. 7. $a p p$. 15 .

1r. CANIS. Dentes Primoves fuperiorès VI: laterales longiores diftantes: intermedii lobati.

Inferiores VI: laterales lobati.

Laniarii folitarii, incuruati. Molares VI. f. VII. (pluresue quam in reliquis.)

familia- I. C. cauda (finiftrorfum) recuruata. Faun. Suec. I2. Syft. ris. nat. 5. Diff. Canis. r. t. r.

Canis. Gefn. quadr: 9r. Aldr. digit. 482. Ionft. quadrs 122. Rai. quadr. 176 . 
10 a. Odobaems. L1735. Odobemo sriso.n56.

1. Rosmarus L.17.5.

Phoca vitulina $\alpha$. noo.

11. anio 2.1735. 

20meftic. $\alpha$. auriculis erectis, cauda fubtus lanata.

fagax. S. auriculis pendulis, digito fpurio ad tilias pofticas. grailts. $\gamma$. magnitudine lupi, trunco curuato, roftro attenuato. Canis fcoticus venaticus. Gefri. quadr. 249. Aldr. 545 .

Molo. Jus. d. magnitudine lupi, labiis ad latera pendulis, corpore torofo. Canis fagax fanguinarius. Gefin. quadr. $25 \mathrm{I}$. aquaticus. \&. pilo crifpo longo, inftar ouis.

meliteus. S. magnitudine Sciuri.

fricator. ท. nafo refimo, auribus pendulis, corpore quadrato.

vertagus. 9. pedibus curuatis, trunco longo fiepius variegato.

auicular. ‘. cauda truncata. Canis pantberinus. Aldr. digit. 555. extrarius. $x$. auriculis longis lanatis pendulis. Aldr. digit. 56r.

negyptius. $\lambda$. nudus absque pilis.

Habitat faepius cum bomine paffun, etiam Jpontaneus ciusfit.

Caput vertice carinatum. Labium inferius lateribus dentatis nudis occultatun. Myftaces ordinibus 5. $\int .6$. Nares extrorfun recuruato finu lunares. Auriculae margine bafeos fuperiore refiexo; pofteriore duplicato; antico trilobo. Verrucae fisciei pilojae. 7. Suturae vellevis 8: collares, fernea, cubitales, abdominales, oculares, lumbares, auriculares, anales. Mammae 10: barum quaduor in pectore. Pedes fibpalmati.

Edit carnes, veget abilia farino fa, non olera. Digerit, ofJa; Vomitu a gramine purgatur, cacut Supra lapidem: $A l$ bum gracum, fepticum funnum. Potat lambendo, sningit ad latus, cunn bofpite facpe centics, odorat anum alterius, odoratu cxcellit nafo bunnido. Lenifime inscedit fippra digitos, vix fudat, calidus linguam exferit, cubitum iturus circumit locum, dormit auditu acutiore, fomniat. Procis rixantibus crudelis, catnllit cunn variis, mordet illa illos, cobaeret copula izmcius; grauida $6_{3}$ diebus, parit faepe 4 ad 8 , mafculis patri similibus, fenineis matri. Fidifinus omnium, docilis, odit ignotos, mordet proiectum lapidem, ad muficam vlalat. Latrat in peregrinos, excepto cane Americae anftralis; a Mabometanis reiectus.

Lupus. 2. C. cauda incuruata. Foun. fuec, 13. Syft. nat. 5 .

Lupus. Gefn. quadr. 634. Aldr. dig. 144. Ionft. quadr. 89. Rai. quadr. 173.

Habitat in Europae $\sqrt{y}$ luis, stiom frigidioribus.

Catere

Cabis genus vorax, morfu lacerans, artores non fcandit; Penis nodofus efi. 
Catcruatim incedit, Pecora, Equos, Sues occidit, rarius bominem; in Jyluis Jufpiciociffmnus vix audet; tractum funiculum metuit. Oculi noctu lucent.

Anatome. E. N. C. d.2.a.9.0bf.7r. et cent. 10.app. 450 .

Hyaena. 3. C. cauda reeta annulata, pilis ceruicis ereetis, auriculis nudis. Syft. nat. 5 .

Lupus marinus. Bellon. aquat. 33. t. 34. Ionft. quadr. t. 57. Gefn. quadr. 674.

Taxus porcinus $f$. Hyaena veterum. Kacmpf. amoen. $4 \mathrm{Ir}$. t. $407 . f .4$.

Habitat in India.

Magnitudo Suis, Pili dorje fubjpitbanaci, erecti, apicibus nigris. Oculi roftro propinquiores. Auriculae mudae. Cauda annulis nigricantibus verticillata; crura anmulis nigris variegata. Striae corporis fufcae et nigrae, transuerfales, a dorfo ad ventren ductae.

Excauat terram degitque in antris, diutius fine cibo viuit, in bumana cadauera faenit, quae e tumulis effodit, coemiteriis infefta.

Vulpes. 4. C. cauda recta: apice albo. Syft. nat. 5 .

Vulpes. Gefn. quadr. 966. Aldr. dig. 195. Ionft. quadr. 82. Rai. quadr. 177 .

Habitat in Europa, Afia, Africa, antra fodiens.

Corpus flan-mufefcens. Aures recti. Maxillae albae. Pedes antice nigrefcentes.

Degit in antris, quae fodit animal aftutiffmum; faeuit in Agnos, Anferes, Gallinas, Auiculas, non Accipitres foetidioresque; colligit ftercora animalium. Fragrat anbrofiaco odore jupra bafin caudae.

Alopex. 5. C. cauda recta: apice nigro. Syft. nat. 5.

Vulpes campeftris vulgo.

Habitat in Europa, Afia.

Lagopus. 6. C. cauda ręta: apice concolore. Syft. nat. 5.

a. Vulpes alba. Kalm. babuf. 236.

ß. Vulpes caerulefcens. Faun. Suec. 14, Habitat in alpibus. Lapponicis, Sibiria.

Pedes denfiffime pilofi wt in Lepore.

aureus. 7. C. Lupus aureus dictus.ot.

Lupus aureus. Kaempf. amoen. 413.t. 407. f. 3. Rai. quadr. I74. 
11 a. Hoyaena. L173s.

1. Hyaena. 2. 1758?

Canis vulpes \& yso.

Canis aloper 2.1350.

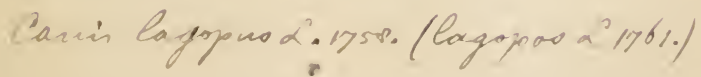

Conins arcusus $\mathcal{L}$. 1ys 


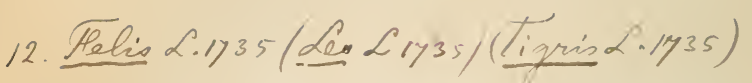


Vulpes indiae orientalis. Valent. mur. $452 . t .452$.

Adill. Bell. itin. $\mathbf{1 6 0 .}$

Habitat in oriente.

Niside flaus; cateruatim incedit, furto deditus; vono clannante clannant remoti alii, ambulante fono nucturno; vlulatus exfecrubilis, quafi eiulatus latratu interceptus. Defcriptio vera animalis etiammun deficit.

12. FELIS. Dentes Primores aequales.

Molaves terni.

Lingua retrorfum aculeata.

$V$ ngmes retractiles.

Leo. I. F. cauda elongaka, corpore heluolo.

Felis cauda elongata floccofa, thorace iubato. Syft. nat. 6. p. 4. n. I.

Leo Gejin. quadr. 572. Aldr. dig. 2. Ionft. quadr. 72. Dodart. act. 1. t. I. et 7. t. 7. Rai. quadr. 162.

Habitat in Africa.

Color luteo-rufus. Frons quadrata. Cor maximum. Miaris Thorax birfutus Caudaque floccofa.

Segnis, efuriens praedatur de Equis et maioribus quadrupedibus, nec'nifi famelicus bomine; fugatur accenfa famma; canibus coercetur. Retro mingit et coit? Rugitus borrendus a tracbeae annulis integris inbricatis.

Anatome. E. N. C. d. I. a. 2. obf: 6. Barth. act. 1671. n. 17 .

Tigris. 2. F. cauda elongata, corpore maculis omnibus virgatis. Syft. nat. 4. n. 2.

Tigris. Gefin. quadr. 936. Aldr. dig. 101. Ionft. quadr. 84. Bont. iau. 52. t. 53. Rai. quadr. 165.

Habitat in Afia.

Magnitudo Leonis. Maculae virgatae, transuer.fae. Pulcherrimum animal trentendac velocitatis, bomini pefsio muts, defolat indos; Mas occidit proprios filios.

Anatome. L. N. C. d. I. a. 2. obf. 7. it a. 9. obf. 194.

Pardus. 3. F. cauda elongata, corpore maculis fuperioribus orbiculatis; inferioribus virgatis. Syjt. nat. 4. n. 3 .

Panthera, Pardalis, Pardus, Leopardus. Gefn. quadr. 824.

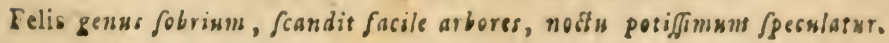


Pardus mas, Panthera femina. Alp. aegypt. 237.t. 15. f.2. Pardalis. Rai. quadr. 166.

Tigris mexicana. Hern. mex. 498. t. 498.

Pinum Dafypus. Nierenb. nat. 153. t. 153 .

Habitat in Inâilis.

Onca. 4. F. cauda elongata, corpcre flauefcente maculis nigris rotundato angulatis medio flauis.

Parchus f. Lynx brafilienfis. Rai. quadr. 168.

Iaguara. Marcgr. braf. 235. Pif. braf. 103.

Habitat in America meridionali.

Pardalis. 5. F. cauda elongata, corpore maculis fuperioribus virgatis; inferioribus orbiculatis. Syft. nat. 4. n.4.

Cato Pardus mexicanus. Hern. mex. 512. t. $5_{12}$.

Catus Pardus f. Catus americanorum. Rai. quadr. 169. Habitat in America.

Magnitudo Melis fupra fufcus, fubtus albicans; lineae punct aque nigraper totunn corpus longitudinaliter Spar$f a$, Sed pedes et abdomen tantum puncis ; latera lineis latioribus albis et fufcis pinguntur. Aures brencs, margine bifidae absque penicillis. Pedes 5-4. Cauda verticillato - variegata proportione Cati. Myftaces 4 ordinum : in fingulo ordine fetae $3 \cdot \int \cdot 5$, albae baji nigrae, lorgitudine capitis.

Catus. 6. F. cauda elongata, corpore fafciis nigricantibus; dorfalibus longitudinalibus tribus; lateralibus fprralibus.

Felis cauda elongata, auribus aequalibus. Fann. Jiiec. 3. Syft. nat. 4. n. 6.

Felis vulgo Catus. Gefin. quadr. 98. Aldr. quadr. 565. Rni. quadr. 98.

Felis. Aldr. dig. 564. Ionft. quadr. 180. t. 72.

Habitat in Europae auftralis Jyluis.

Moribus congenerum, tranquilla, ore molat, casdams erigit; excitata agiliffrma, fcandit, irata fremit odore anbrofiaco, Murum Leo, in praedan intenta caudan mouet, oculi noctu lucent, inbiando praedan baulsit, clamando rixandoque mifere anat. Pupilla interdiu perpendiculari-oblonga, noctu tereti-ampliata; vinguibus complicatis incedit, parce bibit, vrina corrofiut, fercus fepelit, carnes edit vegetabiliaque refpuit, os inftante tempeftate manu lauat, dor fum in tenebris electrifat; in altum acta decidit in pedes. Pulices nan habet. Delectatar Maro, Nepeta, Valeriana. 

13 Yivera 2.1950. 
Lynx. 7. F. cauda abbreuiata, apice atra, auriculis apice barbatis. Felis cauda truncata, colore rufefcente maculato. Faun. fivec. 4. Syft. nut. 4. n. 7. It. Wgotb. 222.

Lynx ceruarius. Giefin. quadr. 677. Tow\%?. it. 2. p. 185. Lynx. Aldr. dig. 90. Ionft. quadr. 82. t. 31. Rai. quadr. 166.

Habitut in Europae fyluis et defertis.

Cauda femoribus panlo breuior apice atra. Palpebra inferior albicans, vt et fuperior verfius cantbum maiorent. Macula linearis fufca pone oculos. Auricularum apices nigri. Palpebrae albae.

\section{VIVERRA. Dentes Primores VI: intermediis breuioribus. Molares plures quam tres. Lingua retrorfum aculeata. Vingues exferti.}

Ichneu. I. V. cauda e bafi incraffata fenfim attenuata.

mon. Meles digitis mediis longioribus: lateralibus aequalibus, vnguibus fubuniformibus. Hafjelqu. itin. I9I.

Meles vnguibus vniformibus, leucophaea. Syft. nat. 6.

Mus pharaonis. Aipin. aegypt. 14.t. 3 .

Ichneumon. Bellon. itin. 96. pifc. 44. Gefin. quadr. 563. Aldr. quadr. 300. t. 301.

B. Mufela glauca. Syft. nat. 5. Amoen. acad. 2. p. rog.

Muftella f. Viuerra indis Mangutia. Kaempf: amoen. 574. t. 567 .

Quil f. Quirpele. Garc. Arom. 163.

Serpenticida f. Muncos. Rimnpf. berb. 7. p. 6y. t. 28. f. 2 . 3 .

Ichneumon indicus. Edw. att. 199.t. I99.

Ichneumon f. Vulpecula zeilanica Seb. muf. I. p. 66. t. 4I. f. $\mathrm{I}$.

Habitat in Aegypto ad ripas Nili, et in India primario; manfucjcit.

Frons plana. Labium fupsrius prominens. Auriculae rotundatae. Myrtaces jimplici feric in omnibus lubiis la. teralibus ad marginem. Lingua fácabra. Pollex a reliquis diftintus. Cauda bafi craffa, Jenfim attenuata, dengitudine corpuris. Folliculus ante aperturam ani, 
fub aefu apertus. Pili albido et grifeo nigrefcente verticillati. Magnitudo Cati, calcaneis inceidit. Victitat muribus, auibus, ouis, ampbibiis, berbis; oua Crocodili effodit et baurit; Serpentum boftis.

Memphi-2. V. cauda fulca luteo-annulata, corpore fpadiceo fubtis. tus flauelcente.

Yquiepatl. Hern. mex. 332. Seb. muf. I. p. 68.t. 42. f. I. Rai. quadr. ISI.

Habitat in America.

Magnitudo Martis. Color fupra albus, fubtus ex albo et nigro variegatus. Pedes talis incedentes. Vngues 5-5: anteriores compreffi, longi; pofteriores breniores, fubtus excauati. Dentes Primores fiperiores in meo nulli; Prinnores inferiores 6, aequales, quorum 2 inteviores; Laniarii fupra et infra vtrinque approximati. Irritata findit cum ventris crepitu balitum foetidifsmum, vt nibil cum eo comparari queat.

Putorius. 3. V. fufca lineis quatuor albidis dorfalibus parallelis.

Putorius americanus ftriatus. Catej'b. carol.2.p.62.t.62. Polcat, Skunk. Fiskatta. Kalm. itin. 2. p.378.

Habitat in America Septentrionali.

Cauda longitudine corporis, villojis.

Irritata vt antecedens balitum explodit, quo nibil foetidius.

Zibetha. 4. V. cauda annulata, dorfo cinereo nigroque vndatim ftriato.

Ciuetta. Cluf. cur. 57 .

Animal Zibethicum. Rai. quadr. 178. Dodart.act.155. Hern. mex. 538. 580. 581. Olear. mi. 7. t. 6. $f .3$.

Hyaena veteribus, nunc Ciuetta. Belion. itin. 94.

Habitat in Indiis.

Corpus oblongum. Roftrum obtufum. Dentes Primores fuperiores 6, paralleli : intermedii paulo minores; inferiores paralleli: medii paullo breniores: alternis interioribus. Laniarii folitarii, strinque diftantes. Molares acuti, denticulati. Aures tectae, brenes. Corporis pili fecundum dorfum longiores, cinereo nigroque vndati, rigidiufculi. Pedes jubtus nudi. Vngues 5-5, canini. Cauda felis annulata.

Vtriculus Zibetbi inter anum et vuluam in vtroque fexu. Anatome. Larth. cent: 4. n. I. et cent. 5. 11. 49. 

14. Unustea L.1735.

14 a. Lictra. 21935.

1. Listra. (Piverra lutra 2.17\%.

1a. Viverra hitreola LilGH. (vide Faum. Suec. p.s?) 
Genetta.5. V. cauda annulata, corpore fuluo-nigricante maculato. $f$

Genetta f. Ginetta. Rai. quadr.20I. Bell. itin. 76. Giefin. quadr. 550.

Habitat in Oriente iuxta rinos.

Caudae annuli 8 nigri; totidemque albicantes. Corpus croceo-nigricans, bene olens, maculis nigris ordine difipofitis; mibi generis objcuri.

14. MVSTELA. Dentes Primores fuperiores VI, erecti, acutiores, diftincti. Inferiores VI. obtufiores conferti: duo interiores.

Lingua laeuis.

Lutris. I. M. plantis palmatis pilofis, cauda corpore quadruplo breuiore.

Lutra marina. Act. petropol. 1749. p. 267.

Lutra brafilienfis. Rai. quadr. 187.

Habitat in Afia et America Jeptentrionali.

Lutra. 2. M. plantis palmatis nudis, cauda corpore dimidio breuiore.

Lutra digitis aequalibus. Faun. fuec. 10.

Lutra. Bell. aquat. 31. t. 32. Gefin. aquat. 515. Aldr. quadr. 294. Dodart. act. 147. t. 147. Rai. quadr 187.

$\therefore \quad$ Habitat in Europae aquis dulcibus, fluniis, Jtagnis, pifcinis.

Victitat pifcibus, ranis, cancris.

Anatome. E. N. C. d. I. a. 3. obf. 195. et d.2. a. 10. obf. II2. ct cent. 10. app. 468.

Gulo. 3. M. plantis filis, corpore rufo-fufco medio dorfi nigro. Faun. Suec. 6. Syft. nat. 5.n. I.

Gulo. O1. Magn. Scbeff. lapp. 339. Racz. polon. 339. Habitat in alpibus Lapponiae; Rufiae, Sibiriae fyluis vafiffimis.

Victitat leporibus, anibus, Rangiferis, cadaucribus, admittit vulpem; voracior vrjo; foetidus; Pollis prac/lantiffima, pretiofiffima.

Anatome. Earth. cent. 4. obj: 30. 
4. M. atra, collo fubtus macula alba triloba.

Habitat in Brafilia. M. Ac. Hoimenf:

Confer. Brown. iam 485. t. 49. J: I. Galera?

Statura Martis, ad nigra: pilis rigidioribus; auriculae rotundae, villofae. Area ante oculos cinerafcens. Maculae fub medio collo, non vero fitb gula. Mammae pone vinbilicun 4 .

Martes. 5. M. plantis fifis, corpore fuluo nigricante, gula pallida. Muftela fuluo-nigricans, gula pallida. Fann. Suec. 3 . Syfl.nat. 5. n. 2.

Martes. Gefin. quadr. 15I. Ionft. quadr. t. 64. Rai. quatr. 200.

Habitat in jyluis antiquis.

Victitat Scinris, Muribus, Auibus.

Putorius. 6. M. plantis fiffis, corpore flatuo nigricante, ore auriculisque albis.

Muffela flauefcente nigricans, ore albo, collari flauo. Faun. filec. 8. Syft. nat. 5. 11. 3 .

Putorius. Gefn. quadr. 767. Aldr. vngu. 330. Ionft. quadr. t. 64. Rai. quadr. I99.

Habitat inter Europae rupes et lupidum acersos.

Noctu praedatur Caniculos, Gallinas. Auiculas, Ous rapit; ano balitu foctidifina.

Furo. 7. M. plantis fifis, oculis rubicundis. $\downarrow$

Muftela fylueftris Viuerra dicta. Rai. quadr. 199.

Hubitat in Africa.

Putorio minor; Auriculae arrectae patulae breules; Oculi rubicundi. Rai.

Zibellina. 8. M. plantis fifis, corpore obfcure fulto, fronte exalbida, gutture cinereo. $\dagger$

Muftela Sibellina. Rai. quadr. 201.

Habitat in Afia feptentrionali.

Color fuluo-objcurus gutture cincreo; anterior pars capitis et auriculae exalbido-cinerene. Rai. Vibriffae ad fupercilia, nares, os longifinnae.

Erminea. 9. M. plantis fifis, caudae apice atro.

Muftela vulgaris. Gicfin. quadr. 149. Aldr. vngu. 307. Ionf. quadr. t. 74. Rai. quadr. I95.

ß. Mu.

Muftelae gerus corpore elongato pedibusque brenibus eft, currit trunco incirruato, fandit arbores, falit ex arbore in arborem. 

Abustela mivalis L. 1YG. (vide Lim. Fanm, Suec. p.y.

15 Urous $x \cdot 13.3$ 
B. Muftela candida f. Ermineum. Rai. quadr. 198.

Muftela alpina candida. Wagn. beluet. 180.

Habitat in Europa et Afia firigidiore; byense praefertim in alpinis regionibus niuca.

Confer. Muftela iauanica. Seb. muS. I. p. 77. t. $48 \cdot f \cdot 4$. Cauds dimidis extrorjim nigra; corpus byeme album, aeftate rufum : excepto abdomine, pectore, gula. Pellis pretiofa bodie feminis noftratibas.

Edit Pifies, carnes, Mures, oua, Fungos, non regetabilia; nutriffenu, foetida, fiticuloja, noctu praedatur 3 terrefacta epilepfia corripitur.

is. VRSVS. Dentes Primores fuperiores VI, intus excauati alterni.

Inferiores V I : laterales 2 longiores lobati; fecundarii bafi interiores.

Lamianii folitarii, conici. Molares V f. VI: primo laniariis approximato.

Lingua laeuis.

Membrana nictitans. - Nafus prominens.

Penis fcatens offe recuruato.

Argos. I. V. cauda abrupta. Faun. fuec. 2. Syft. nat 4. n. r. Vrfus, Gefin. quadr. 14. Aldr. digit. 117 . Ionft. quadr.

t. 55. Dodart. act. 67. t. 67. Rai. quadr. 171 . Habitat in fyluis Europae frigidae.

Irrequictus; abftemius bybernat intra Mufcos; lambit pedes (Barth act. 1674 p. 34.); infiat praedam.

Virfus maritimus albus maior, ar.ficus. Martens. Spitzb.

73. t. O. f. C. forte diftincta Jpecies eft, nobis non vifa.

lufcus. 2. V. cauda elongata, corpore fernugineo, roftro fufco, fronte fafciaque laterali longitudinali albida.

Vrfulus lupo affinis americanus. Edw. aw. I.p. 103.t.103. Habitat ad finm Hudfonis. 
Meles. 3. V. cauda concolore, corpore fupra cinereo fubtus nigro, fafcia longitudinali per oculos auresque nigra.

Meles vnguibus anticis longifimis. Fram. Juec. 15. Syft. nat. 6.

Meles. Gefn. quzdr. 686.

Taxus. Aldr. digit. 264. Ionft. quadr. t. 64. Rai. quadr. 185.

Habitat in Europa inter rimas rupium et lapidum.

Venatur cuniculos, antra fodit inbabitatque; fiercus in eodem loco depununt inbabitantes extra antrum; die latet, corradit Lathyrum in byemem, folliculum putorium supra anum baw it bybernans.

Anatome. E. N. C. d. 2. a. 5. obj: 32. et d. 3. a. 3 . obj: 163 .

Lotor. 4. V. cauda annulata, fafcia per oculos transuerfali.

Vrfus cauda elongata. Syft. nat. 4. Act. Stockb. 1747. t.9. $f$. $\mathbf{~}$.

Mus indicus alius. Gefin. quadr. 74I.

Vulpi affinis americana. Rui. quadr. I79.

Raccon. Kalm. it. 2.p. 228.

Coati-monde. Marcgr. braf. 228. Ionft. quadr. I39. Worm. mif. 319. Dodart. act. I8I. t. I8I. Maior. mofc. 30. Rai. quadr. 180.

Felis montana americana. Seb. mul. I. p. 68. t. 42. $f$. $\mathbf{\text { . }}$

Habitat in Americae mavitimis.

Cauda craffa, ferruginea annulis nigris.

Edit lubenter oua, Gallinas; cibum lauat; odoratu ta. efuque excellit; mentis tenaciffinus, dormit a 12 noctis in 12 diei; pellitur Jetis porcinis. Penis offe incuruato fatet. 
is a. Sheles. 2.1735 
v. Bestiae L ysos.

16. Sus. L.1735. 


\section{BESTIAE.}

Dentes Primores vtrinque: intermediis diftantibus.

Laniarii femper vno plures vtrinque:

Nafus vitra os prominens.

16. SVS. Dentes Primores Superiores IV, conuergentes. Inferiores VIII patuli. Laniarii Superiores II breniores. Inferiores II exferti.

Scrofa. 1. S. dorfo antice fetofo, cauda pilofa. Fn. fuec. 36 . Syft. nat. 12.

Aper. Gefn. quadr. I46. Aldr. bifulc. 1013. Ionft. quadr. 74. Rai. quadr. 92.

B. Sus. Gefin. quadr. 872. Aldr. bifill. 937. Ionft. quadr. t. 47. Rai. quadr. 92.

$\%$ Sus chinenfis. It. Wgotb. 62. It. Scan. 72.

Habitat in Europa anjtraliore.

Aper vix differt a Sue domejtico, nife quod lanam inter jetas non babeat.

Chinenfis dorfo fibnudo, abdomine ad terram vsque dependente differt.

Varietas frequens Vpfaliae Suis domeftici femper monunguli, in caeteris eadem jpecies.

Animal odore pollens, navibus fodiens: tendinibus truncandis; quisquilias, ftercus, fucculenta, variaque Spurcata vorans; multa filigens; pinguiffrnum, Jlupidun, Jomnulentum, tempeftatum praenuncium et impatiens; apricatur in fole, in cocno $\int_{e}$ volutat, immundum, grumiens, accurrit clamanti aduerjis boftems Spumante ore dentibusque borrens, Crotalis infeftum; tardun venere, pene laxo longo; mammis numerofiffrnis, prole innumera; laborat pediculis, fcabie, foropbulis, pipere. Ganeae numerofa materies, Atbletis. et exercitatis falubvis, non vero criticis; faccbaro j: ficu inprimis altilis laudatur : inde Pernae, Syncipitia, Trunculi, Coflae, Sumina, Vuluae, Ilicia, Botuli, Iufcula; at fiercus in fiuxu fanguinio valct. 
Porcus. 2. S. dorfo poffice fetofo, cauda longitudine pedum, vmbilico cyftifero. Syft. nat. I2.

Porcus guineenfis. Rai. quadr. 96 .

Habitat in Africa.

Affinis priori. Corpus rufum. Auriculac acuninatifimate.

Cauda longa, nuda.

Tajacu

Vellus fetis caret, nifi fupre clunes.

Iaiacu. 3.S. dorfo cyitifero, cauda nulla. Syft. nat. 12.

Taiacu. Pif. Ind. 98 .

Taiacu f. Aper mexicanus mofchiferus. Tyfon. act. 32. 153. Rai. quadr. 97.

Cuaiguara. Marcgr. braf 229. Bartb. cent. 3. n. 44. Quanlifla cogmati Quapızott. -Hern. mex. 637.t. 637.

Habitat in Mexici, Panamae, Brafiliae montibus, fyluis; edit Ranas, Serpentes.

Corpus cinerenn; farcia jiaul circum buneros; crux nigra Jupra nafuni.

Pedes nigri macula alba fupra genua antica. Setae fa. Atigiatae nigrae inter breutes aures, nec in dorfo. Protuberantia ad latera oris et fupra nafunn. Glandula fecretoria Supra clunes, fecernens odoratum liquoren ambrofiacum. Z. Hallman.

Babyruf-4. S. dentibus duobus caninis fronti innatis. Syft. nat. 12. fa. Babyrouffa. Bont. inu. 16. Barth. cent. 2. obj: 96. Grew. mul t. I. Seb. mul. I. p. 80. t. 50. f. 2. Rai. quadr. 96.

Habitat in Borneo Indiae orientalis.

Magnitudine Cernit. Dentes Laniarii fuperiores II, perforantes cranium frontis, recumati vt 2 cornua; inferiores virinque folitarii, magnii, adftant prioribus.

7. DASYPVS. Dentes....

Corpus tefta offea cataphractum, Zonis interfecta.

vnicin- I. D. tegmine tripartito, pedibus pentadactylis. Syft. nat.6. Etus. Taru f. Armadillo africanus. Seb. mus. r. p. 47.'t.30. $f: 3.4$.

B. Dafypus cingulo fimplici. Syft. nat. 6.

Tatu muftelinus. Rai. quadr. 235. Grew. mus. 19. t. I. Habitat in Africa. 
16. Hydurohaerees Bress. 1956.

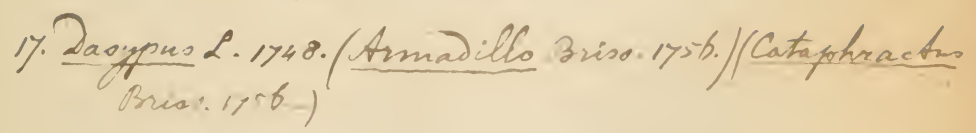



tricinEtus.

quadricinctus.
2. D. cingulis tribus, pedibus pentadactylis.

Dafypus cingulis tribus. Syft. nat. 6 . Tatou f. Armadillo. Rea. exper. or. t.92.

Tatu f. Armadillo orientalis. Seb. muj: I. p.62.t. 38 . f. 2.3 .

Tatu apara. Marcgr. braf. 232. Pif. braf. roo. Rai. quadr. 234.

Armadillo f. Tatou genus alterum. Cluf. cxot. 109. Armadillo. Grew. muf. 17. Olear. muj. 8. t. 6. f. 4 . Habitat in India orientali.

fexcin- $\quad 4 . \mathrm{D}$. cingulis fenis, pedibus pentadactylis. Muf. Ad. Fr:? हtus. Dafypus cingulis fex. Syft. nat. 6. Tatu f. Armadillo primus. Rai. quadr. 233. Tatu f. Tatu-pera. Mavegr. braf: 23r. Armadillo f. Tatou. Cluf: exot. 230. Habitat in America meridionali.

feptem- 5. D. cingulis feptenis, palmis tetradactylis, plantis pencin\&us. tadaktylis. Amocn. acad. I. p.28I. Dafypus cingulis feptem. Syft. nat. 6. Habitat in Indiis. Ais Jufficienter a jequente difinctus?

nouem- 6. D. cingulis nouem, palmis tetradactylis, plantis pentam cinctus. dactyiis. Muf. Ad. Fr. 6.

Dufypus cingulis nouem. Syft. nat. 6 .

Tatou 1. Armadillus americanus. Seb. muf. I. p. 45.t. 29. f. r. et t. 53. $f \cdot 6$.

Tatue brafilienfibus. Marcgr. Uraf. 23r. Rai. 9. quadr. 233.

ค. Dafypus. Hirn. mex. 314.

Habitat in America meridiunali, 
Is ERINACEVS. Dentes Primores II, paralleli, fubcuneati, longiores, diftantes: fuperiores remotiores.

Lnniavii II vtrinque, antrorfum decumbentes, parui.

Molares III f. IV acuti lobati.

Corpus fpinis obtectum.

europaeus.I. FrinaCEvS. Fn. Jit. 16. Syft. nat. 6. It. Goth. 264. Echinus f. Erinaceus terreftris. Gefin. quadr. 368. Aldr. quadr. 459. Ionft. quadr. IIY) t.68. Rai. quadr.23I. Sel. muif. 1. p. 78. t. 49. f. 1. 2.

Habitat in Europa.

Mitis, ambrofiacus, polyfbagus, nocturnus, monogamus, bybernans, nidificans e Mufco in fruticibus, metu fe contrabens, naribus criftatis.

Anatume. E. N. C. d.2. an. obf. 65. et an. 6. obf. 105.

et cent. 10. app. 447. Barth. act. I671. n. 90.

19. TALPA. Dentes Primores fuperiores VI. Inferiores VI]I.

Laniarii maior I, et minores IV.

Molaves acuminati.

Europaea. I. T. caudata, pedibus pentadactylis.

Talpa caudata. Syft. nat. 7. Faun. Suec. 17. It. Scan. 332.

Talpa. Gefri. quadr. 931. Aldrou. digit. 451. Rai. quadr. 236. Seb. mus. I. p. 68. t. 41. fig. I, 4 .

Habitat in Europae bumofis fub terra, victitans e Lumbricis, non plantis, noctu praedatir.

Anatome. E. N.C. d. I. a 2. obj: 51. ct d.2. a. I. obj. I30.

Penis omnium aninalium longiffimus. Peilis delicatula.

2. $\mathrm{T}$. 
10. Erinacens 2.1735.

9. Savpa 2. 17s5.. 


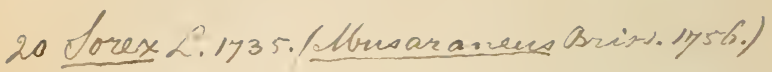


afiatica. 2. T. ecaudata, palmis tridantylis.

Talpa fibirica verficolor. Seb. muf. I. p. 5I. t. $\hat{\jmath}^{2}$. $f: 4,5$.

Habitat iн Sibiria.

20. SOREX. Dentes Primores Superiores II, bifidi. Inferiores IV: intermediis 2 brenioribus. Laniarii vtrinque plures.

Araneus. I. S. cauda corpore longiore.

Sorex. Faun. fuec. 33.* Syft. nat. 10.

Mus araneus. Gefin. quadr. 741. Aldr. digit.441.t.442. Ionft. quadr. t. 66. Rai. quadr. 239.

Habitat in Europae cryptis.

Auriculae brenes, amplae. Cauda longa.

criftatus. 2. S. naribus criftatis, cauda corpore breuiore.

Habitat in Penfyluania. P. Kalm.

Facics Talpae, colore atro, palmis nimeis.

Auriculae nullae. Roftrum longiffenum. Cauda fubnuda, corpore dimidio breuior, concolor. Nares fingalae Jtella palmata radiis $10 \int 15$. Jubulatis, nudis vt in Erinaceo. Dentes primores fipperiores 2; inferiores 4. Laniarii vtrinque flures.

aquaticus. 3. S. plantis palmatis, paimis caudaque corpore breuiore albis.

Talpa virginianus niger. Seb. mul. I. p. 5 I. t. 32. $f \cdot 3$.

Habitat in America feptentriunali. $P$. Kalm.

Facies praccedentis, jed cinevea aut fujca, palmis albis.

Cauda corpore quadruplo brenior, alba. Dentes primores juperioris $2 \%$ inferiores $4:$ intermediis breuifinis. Laniarii vtrinque plures, difcreti.
D 5
2I. DI.

Sorex capenfis maximus Pet. gax. 37. t. 23. f. 9. nobis non :ifus ef.. 
21. DIDELPHIS Dentes Primores Superiores X. Inferiores VIII: intermeciis 2 breuifimis. Laniarii longi. Molaves denticulati.

Lingua fubciliata.

Foliculus abdominalis mammarum.

marfu- I. D. mammis 8 intra abdomen. Amoen. acad. I. p. 279. pialis. Syjt. nat. 10. Muf. Ad. Fr. Iо. Philander. Seb. muf. I. p.64.t. 39 .

Opoflum. Tyfon. act. 290. p. 1565.

Carigue. Laët. amer. 55 I.

Cariegueya brafilienfibus. Marcgr. braf. 222. Pif. braf. 323. Ionft. quadr. 136. t. 63 .

Maritacaca. Pif. braf. 323. Rai. quadr. 182.

Tlaquatzien. Hernand. mex. 330.

Habitat in America.

Corpus Melis, magraitudine Felis maioris. Roftrum conicum, lsngun Suis inftur, carinatum, rictu longiffimo Vulpis. Nares perpendiculares, lunares. Vibriffae 5 ordinum, longae; fetae pone oris rictum 8, fub gula 5. Auriculae rotundatae, atrae apicibus albis. Dentes molares lobati: anteriores fimplices: primi minimi; reliqui vt in cbaractere. Tibiae nigrae, laeives pilis brevibus. Cauda muda, longitudine corporis, quafi fegmentis ex rugis, prebenfilis. Manus pentaddEtylae, fiffae. Digiti teretes, mudi, aequales. Vngues compre $\sqrt{f}$, arcuati. Plantae vt in palmis: Pollex omzinino muticus.

Pullos lactantes territosque intra abdonen secipit mater.

Philan- 2. D. cauda bafi pilora, auriculis pendulis, mammis quader. ternis.

.Tlaquazin f. Tai-ibi braflilienfibus. Seb. minf. I. $p$. 5\%. t. $36 . f .4$.

flubitat in America.

Figu-

Didelphis polite plantemsm, ati diftinto fo remoto, gaudent Primates motico. 
21. Didelphis L1yss. (Ohilander Bu'ss. 1'st.) 

Figura Sebae aliena roftro rotundiore, fafcia fufca trans oculos, follice poftico anguiculato, quod non in noftro. Vbera dus: mamnis in fingulis 2.

Opof- 3. D. cauda femipilofa, fuperciliorum regione pallidiore, fum. mammis binis.

Philander, Opoffum f. Carigueia. Seb. muf. r. p. $56 . t$. 36. f. I. 2.

Habitat in America.

Abdomen circa mammas contrabitur in marfupium; pollices poftici mutici.

murina. 4. D. cauda femipilofa, mammis fenis. Amoenit. acad. l. 279 .

Mus fylueftris americanus. Seb. mul. r. p. 48.t. 3r. $f$. 12. 36.

Habitat in Afia, America.

Vngues digitis ommibus acuti, exceptis pollicibus pofticis. An pullus praecedentis?

dorfigera. 5. D. cauda bafi pilofa corpore longiore, digitis manuum muricis. $\dagger$

Mus fylueftris americana. Seb. muf. I. p. 49. t. 3I. f. 5, 4. et 2. p. 90. t. 84. f. 4. Merian. Jirrin.

Habitat in America.

Pullos in dorfo gerit caudis cirrbi inftar conuolutis. 


\section{GLIRES.}

Dentes Primores bini fupra it infra, a molaribus remoti.

Laniavii milli.

\section{RHINOCER OS. Dentes Primores II.} Cormu nafo infidens.

vnicor- I. R. cornu vnico conico. Syft. nat. II. $n$. I.

nis. Rhinoceros. Ionft. quadr. 98. t. 38. Bont. iau. 50. t. 51. Bergen. orat. 1746. t. I. Gejin. quadr.842. Aldr. bifulc. 878 .

Habitat in Africa, India, coeno fe volutans, viftitans vepretis.

Statura prioris fere Elephantis.

Cornu in roftro, folidun, recuruum, perpetum, conicun. Labium fuperius acuminatunn aduncuill.

Oculi ct Aures paruae.

Cutis nuda. Rugae transuerfales: I. Capitis poftica. 2 Scapularis: 3. Abdominalis ante fenora. 4. Femoralis trans femora. Vngulae tres.

Cauda attenuata pedibus brevion.

Animal mite, ni lacefjatur, tum arbores fragore profernit, cornu eiicit omnia.

Quantus erat cornu, cui pila Taurus erat. Martialis.

bicornis. 2. R. cornibus duobus cuneiformibus. Syft. nat. II. $n .2$. Habitat in India Kolbius, Iacobaeus, Scbroeckius.

Species obfirura, cranium tantum cum cornibus duobus - compreffis altero miarori fupra alterum nobis vifun.

23. HYSTRIX. Dentes Primores II oblique 'fciff. (Rai.)

Molaves octo.

Corpus fpinis tectum.

criftata. I. H. palmis tetradactylis, plantis pentadactylis, capite criftato, cauda abbreuiata. Syft.nat.9. n.I. 
v. Glires 21735.

22. Rhinoceros. L. 1940.

23. Hopotin . X. 1735: 
24 Lepens L. 1735. 
Hyftrix. Gefin. quadr. 563. Aldrou. digit. 471. Ionff. quadr. niq. t. 68. Dodart. act. 233. Rai. quadr. 206. Hyftrix orientalis criftata. Sel. muf.: I. p. 79. t.5०. f. I. Habitat in Afia.

prehenfi- 2. H. pedibus tetradactylis, cauda elongata prehenfili fe-

lis. minuda. Syft. nat. 9. n. 2.

Hyltrix. Bont. iau. 54.

Cuandu brafilienfibus. Marcgr. braf. 233. Pif. asmer. 99. Rai. quadr. 208.

Hoitztlacuatzin. Hern. mex. 322.

Habitat in Afia, America meridionali.

dorfata. 3. H. palmis tetradątylis, plantis pentadactylis, cauda elongata, dorfo folo fpinofo.

Hyftrix canadenfis. Eltiv. all. 52. t. 52.

Habitat in Canada.

Corpus ferrugineum, Canda apice fübtus alba.

macroura.4. H. pedibus pentadątylis, cauda elongata : aculeis clauatis. Syft. nat. 9. n. 3.

Porcus aculeatus f. Hytrix orientalis. Seb. muf. I. p. 84. t. 52. f. $\mathrm{I}$.

Habitat in Afia.

brachyu- 5. H. pedibus pentadactylis, cauda abbreuiata. Syft. nat. ra.

9. n. 4 .

Porcus aculeatus f. Hyftrix malaccenfis. Seb. mulf. I. p. 8I. t. 5I. f. I.

Habitat in Afia.

24. LEPVS. Dentes Primores II : fuperiores duplicati: interioribus minoribus.

timidus. I. L. cauda abbreuiata, auribus apice nigris. Syft. nat. 9 . n. 2. Faun. Jucc. 19.

Lepus. Cicfir. quadr. 69. Aldr. dig. 247. Ionft. quadr. t. 65. Rai. quadv. 204.

Habitat in Europa.

Hycme in frigidlis niueus, cxceptis auribus nigris. Cauda etiam aeftate alba. Aures longae. Oculi exftantes membrana niçitante, jönno aperti. Femora poffica crafja. Pedibus lanatis 5-4, et dorfium conffitutunt

pras-

Lepus cornutus, fi non bybridus, certe fabulofus fit. 
pracftantiffnam partem. Nares bumidi, motitantes. Inermis curfiu valet, tinnidus, currit gyros fenfin breuiores, vetro deuiat exfiliendo; a Canibus, Felibus, Accipitribus, Noctuis venatur; cauitas vtrinque e regione pubis; ceruice fragili; immenfus parturitione; vrina foctida non in cubile; pulicibus obnoxius.

Anatome. E. N. C. d. I. a. 2. obf. 251. et a. 3. obf. 93 . et d. 3. a. 5. obj:225. Partb. act.1671. n. 136.

Cunicur. 2. L. cauda abbreuiata, auriculis nudatis. Syft. nat. 9.n. lus. 3. Fann?. Jitce. 20.

Cuniculus. Gijin. quadr. 72. Aldr. digit. 382. Ionft. quadr. t. 65. Rai. quadr. 205.

Habitat in Europa auftrali, antra fodiens in arenofis. Hoftes ip $\sqrt{2}$ Falcones, Meles, Putorius.

Species facilius dignofcitur, quan differentia Specifica determinutur.

Anatome. Partlobf. 10.

capenfis. 3. L. cauda longitudine capitis, pedibus rubris.

Habitat ad Cap. b. Spei. Fodiens. 1. Burmanmus.

brafilien- 4. L. cauda nulla. Syft. nat. 9.n. T.

fis.

Cuniculus brafilienfis Tapeti. Marcgr. braf. 223. Rai. quadr. 205 .

Habitat in America meridionali.

25. CASTOR. Dentes Primores fuperiores truncati, angulo transuerfo excauati; inferiores apice transuerfi.

Canda depreffa.

Fiber. x. C. cauda ouata plana. Faun. Succ. 23. Syft nat.'io. n. I.

Caftor. Gefn. pifc. 185. Rond. pifc. 236. Ionft. pifc. t. 68. Dodart. aEt. 13I. Rai. quadr. 209. Aldr. quadr. 706.

Fiber. Bellon. aqu. 30.

Habitat in Europae, Afrae, Americae borealibus ad ripas funiorun lacummque, victitans corticibus imprimis Populi tremulae; Domos mive exftruit. 
$\therefore$ Costor. x? ys. 


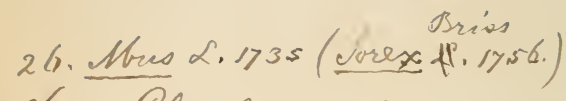

$26 \cdot a$. Glis. Ariso. 1956.

26 6. (Amioules Briss.1756.) Cavia Klem,1751. 
Oeconomia animalis data a D. Ciflero, act. Stockl. $175^{6}$. p. 207 .

Anatome. E. N. C. d. 1. a.2. obf. 25 r.

mofcha- 2. C. cauda longa lanceolata plana. Faun. fuec. 24. Syja. tus. nat. 19. 11.2.

Mus aquaticus exoticus. Chuf. exot. 375. Worm. mul.. . . Rni. quadr. 217.

Ilabitat in Rufiae aquofis, America.

Pedes poftici, vt in antecedente palmati; vicfitat Acoro.

\section{MVS. Dentes Primores inferiores fubulati.}

Porcel- I. M. cauda nulla, palmis tetradactylis, plantis tridactylus. lis. It. Wgotb. 224. Syft. nat.10. n. I.

Cuniculüs indicus. Gefin. quadr. 367. Aldr. digit.391. 993. Porcellus indicus. $f$. Cuniculus indicus. lonft. quadr. I6r. $t \cdot 63 . f 6$.

Mus 1 : Cuniculus americanus guineenfis, porcelli pilis et voce. Rai. quadr. 223.

Cauia Cambaya. Marcgr. braf. 224. Pif. braf. 102.

Habitat in Brafilía.

Hiftoria buins babctur iu Differtatione de Mure indico.

Conf. Capybara. Marcgr. braf. 230. buic affinis, fed maior, coloris ignoti.

Pipientes, loquaces, inquicti, fequaces, calcitrantes, attenti, comantes, majlicantes, edunt veretubilia variata; potant lymplam puram, calorem amant; femina mamnis duabus, parit pullos perfectifimos, mox coit; mas bombylans imiures mafculos mordet paraplefit. cos. Color zarius eticum Spontaneis.

leporinus.

2. M. cauda abbreuiata, palmis tetradaatylis, plantis tridutylis.

Lepus ianenfis. Catesb.carvl. 3.p. IS. t. IS.

Habitat in Iaua, Sumatra.

Aures fubrotzundac, Color rufus, abdomine albo.

Lem- 3. M. cauda abbreuiata, pedibus pentadactylis.

mus. Mus cauda abrupta, corpore fuluo nigroque vario. Fann. fucc. 26. 1.7. Stockb. 1740. p. 326. t.6. f. 45 . Syjt. nat. 10. $n .2$.

Mus montanus. Scheff: lapp. 34 6.

Lemming. Olear. muj: 21. t. 12. f. 6. 
Lemmus. Ol. Magn. fept. 1820.

Mus noruagicus. Worm. muf. 322. t. 325. monogr. 6. Rai. quadr. 227.

Habitat in alpibus Lapponicis.

Gregatim fugiunt quibusdam annis ex alpibus, recta via iter inftituentes; edunt Licbenem rangiferimum, amenta Betulae nance, non carnes.

Wormii Hifloria Muris noruagici. Haffin. 1653. quart. bunc fiftit.

Anatome. Barth. cent. 2. p. 301 .

Marmo- 4. M. cauda abbreuiata fubnuda, auriculis rotundatis, buc-

ta. cis gibbis.

Mus cauda elongata nuda, corpore rufo. Syft. nat.10. $n$. II.

Mus montanus. Mattb. dio fc.2.c.63.

Mus alpinus. Rai. quadr. 22I. Wagn. belu. I79. Dodart. mem. 3. p.31. t. 3I. Gefr. quadr. 743. Aldr. quadr. 445 .

Habitat in alpibus Helueticis, Tridentinis.

Dentes primores inferiores apice dinergentes.

Myfaces vtrinque ferie fextuplici. Aures paruae, vefitae. Bucca virinque: admodum gibba. Cauda palmaris, nigra. Verruca fupra oculos fetis 6 ; jub oculis fetis 7 . Dorfum cinereum. Inguina et pedes ciquereo flauefcentia. Sutura a gula ad anum. Pedes 4-5. H. Z. Hallman.

Profunde et celervine fodit; bybernat alto fomno, ma. nibus cibum ori aulnouet, faepe erecta incedit; facilius in planitie, quann fub terra capitur.

Monax. 5. M. cauda mediocri pilofa, corpore grifeo, auriculis fubrotundis, palmis tetradactylis, plantis pentadactylis. Monix f. Marmota americana. Edw. au. 104. t. I04. Habitat in America feptentrionali.

Cricetus. 6. M. cauda fubabbreuiata, auriculis rotundatis, corpore fubtus nigro, lateribus rufefcentibus.

Mus cauda elongata, corpore cinereo rutilo nigroque longitudinaliter vario. Syft. nat. 10. n. 10.

Cricetus. Rai. quadr.221. E. N. C. d. 2.a.5.0bJ. 186. Gefn. quadr. 738 .

Hamiter. Meyer. tbiere (Norib. 1748. fol.) t.81. 82.

Habitat in Germania. 

Nows agrestis L. 176s. (vide Farm. Swec. p. 11.) 
Numerus digitorum pedunn in bac st. antecedents nobis ignotus. Cuniculo minor, cauda pedibus fere breuior. Bucca vtrinque faccata.

Cauernas fodit jub terra plurimis concamerationibus, - duplici foramine: altero obliquo aditu, altero perpendiculari Specula. Colligit Frumentum, Pifa, Fabas, fructus, in diftinctis cameris firuanda, adportat ea in bucca; parit bis in amno Jex pullos, itidem in propria camera feruandos, wti stiam fierquilinum in diftincta.

Venatur ob carnes, pelles, frumentumn collectusni; occiditur pulueve Veratri, farina et melle in panes coctis. Citillus auctorum, fi ab boc diuerfus, probe obferuandiss,

terreftris. 7. $M$. cauda mediocri fubpilofa, palmis fubtetraductylis, plantis pentadactylis, auriculis vellere breuıribus.

Mus cauda longiffima pilofa, auribus fubrotundis vellere breuioribus. Fn. fiec. 29. Syft. nat.pio. n.5. Ed v/.

Mus agreftis, capite grandi, brachyuros. Rai. quadr. 218 .

Hahitat in Europae terra et aqus.

Corpus fufcum fubtus palliduin, at non albicans, Caput craffius, ore gibbo. Cauda magis pilofa, quam in Ratto, fid corpore dimidio brenior, at pedibus fere longior. Hortos Talpae inftar milere effodit, paimis licet paruis; natat in foffis et vrinatur plant is licet filfis; Rad ces arbornm decorticat, plantarum confumit $\int$ anfert; Pullos anatum in pifcints occidit.

amphi- 8. M. cauda elongata pilofa, plantis palmatis. $\dagger$

bius. Mus maior aquaticus $f$. Rattus aquaticus. Rai.quadr, 217.

Mus aquaticus. Bell. aquat. $35 \cdot t \cdot 36$.

Habitat in Europae, Africae fo/fis, ripis, pifcivis, bortis.

Species mibi non rite cognita.

Fodit ad folfas et ratices arbormm, natat, vrimatur, confunst radices, bortis ef fut is infefius, capitur Nafjis e virgulis confectis fub aqua demeryis.

Rattus, 9. M. canda elcingáta fubnuda, pilmis rerradactylis cum vnguiculo pollicari, plantis pentadaktylıs. 
Mus cauda elongata fubnuda, corpore fufco cinerafcente. Faun. Juec. 28. Syfu. nat. 10. n.6.

Mus domefticus maior. Gefn. quadr. Iog. Aldr. quadr. 417. Rai. quadr. 217 .

Glis. Ionft. quadr. f. 66.

Habitat in domibus Europae.

Cautum animal, vtenfilibus infeftum.

Glirem veterum me ignorare agnofio, nifi fit Marmotasut Cricetus.

Mufcu- Io. M. cauda elongata fubnuda, palmis tetradactylis, planlus. tis pentadątylis.

Mus cauda nudiufcula, corpore cinereo-fufco, abdomine fubalbefcente. Faun. Jüec. 3I. Sylt. nat. n. 8 .

Mus. Gefir. quadr. 7I4. Aldr. quadr. 417. Ionft. quadr. t. 66.

Mus domefticus vulgaris $f$. minor. Rai. quadr. 218.

Habitat in domibus.

Vnguis pollicaris palnzarum nullus, quo a Ratto differt. Delectatur mufica; non facile incarceratur; polyphagus.

ăellana- II. M. cauda elongata pilofa, corpore rufo, gula albicante. rius. Fann. Jivec. 32.

Mus auellanarum minor. Rai. quadr. 220. Aldr. digit. 440.

Habitat in Europae fylivis.

Colligit muces, glandes; Jiperfuas defodit.

fyluati- 12. M. cauda longa, palmis tetradactylis, plantis pentacus. dactylis, corpore grifeo pilis nigris, abdomine albido. Faun. fuec. 30. Syjt. nat. ro. n. 7 .

Mus domefticus medius. Rai. quadr. 218 . Habitat in bortis et fyluis Europae.

Afjeres duriffinos, juepe vno die perforat.

ftriatus. I3. M. cauda elongata fubnuda, palmis tetradactylis, plantis pentadastylis, corporis ftriis punctatis.

Mus cauda longa, ftriis corporis longitudinalibus et punEis albis. Mur. Ad. Fr. Io.*

Mus orientalis. Seb. muf. 2. p.22. t.21.f. 2. Habitat in India.

longipes. I4. M. cauda elongata veftita, palmis tetradactylis, plantis pentadartylis, femoribus longiffumis. 

2). Scinnus 2. 1935 
Mus cauda longa veftita, pedibus pofticis longitudine corporis flaui. Muf. Ad. Fr. 9.*

Faculus Habitat in torridis regionibus.

Iaculus. 15. M. cauda elongata floccofa, palmis pentadactylis, plantis trid_etylis, femoribus longifimis, brachiis breuiffimis.

Mus iacullus pedibus pofticis longifimis, cauda extremo villofia. Haffelq. itin. 198. act. Stock. 1752. p. 123. t. 4 . f. $\mathrm{r}$.

Mus aegyptius. Hafelq. act. Vpf. 1750 . p. 17 .

Cuniculus minor caudatus dauricus. iniuf: Petrop. I. p. 344. n. 123 .

Cuniculus f. Lepus indicus Vtias dietus. Aldr. quadr. 395 fir. bon.

Lepus cauda elongata. Syft. nat. 9. n. 4 .

Habitat in Arabia, Calmukia.

I'lantis tantun incedit, et quiefcit; palnnis cibum ori adinoulet.

volans. I6. M. cauda elongata villofa, palmis tetradactylis, plantis pentadactylis, latcribus extenfi, volitans.

Sciurus americanus volans. Rai. quadr. 215. Seb. mus. I. p. 72. t. $44 . f .3$. Edw. aul. 19I. t. 19r.

Habitat in Virginia, Mexico.

Cutis ab auribus ad bracbia et fensora ct caudan ex. tenfa, margine plicato nigro.

27. SCIVRVS. Dentes Primores II: Superiores cuneat1;

Inferiores compreiri.

vulgaris. I. S. auriculis apice barbatis, palmis tetradaetylis, plantis pentadactylis.

Sciurus palmis folis fal iens. Faun. fuec. 21. Sy.f. nat. 9. n. I. Sciurus. Gefin. quadr. 13. Aldr. quadr. 398. 10nffo quadr. t. 66.

Sciurus vulgaris. Rai. quadr. 214.

Habitat in Europae arboribus.

Aeftute ruber abdomine albo, byeme cinereus. Tictitat mucibus, frobilis erc. Jedens. Superfunum cibums defodit; Cortice interdum namigat. Nidus globojus e mujcis.

Anatome. E. N. C. cent. 10. app. 449. 
niger. 2. S. niger. Catésb. carol. 2. p.73,t.73. Habitat in America fiptentrionali.

cinereus. 3. S. virginianus cinereus maior. Rai. quadr. 215. Catesb. car. 2.p.74. 174. Kalm. itin. 2. p. 409.

Habitat in America feptentrionali.

Magnitudo tripla aut quadrupla Sc. vulgaris.

Hauus. 4. S. auriculis fubrotundis, pedibus pentadactylis.

Sciurus cauda tereti, pilis breuibus, auriculis fubrotundis. Amoen. acad. I. p. 2SI.

Habitat in America.

Magnitudine dimidio minor Sc. vulgari, luteus, apicibus pilorum albis. Pollex anticus folo fere vngue conftat.

getulus. 5. S. fufcus, ftriis quatuor albidis longitudinalibus.

Sciurus getulus. Rai. quadr. 216. Edw. au. 198. t. 198 . Seb. muf. . p. 76. t. $47 \cdot f \cdot 3$. Habitat in Africa.

ftriatus. 6. S. flauus ftriis quatuor fufcis longitudinalibus. Muf. Ad. Fr. S. *

Sciurus friatus. Catesb. car.2. p.75, t.75. Edwo. als. I8r. $t$. $18 \mathrm{I}$.

Roeffelvifla. Kalm.itin. 2.p.4r9.

Hobitat in America feptentrionali fub terra.

Mngnitudine Mirris musculi vel paulo maior. Palmae tetradactylae, plantae pentadactylae.

volans. 7. S. hypochondriis prolixis volitans. Faun. fucc. 22. Syft. nat. 9.

Sciurus volans. Seb. mif. I. p. 67.t.41. f. 3. Catesb. car. 2. p. 76. et 77. Edw. au. I91. $t$. 191.

Mus ponticus f. fcythicus. Gefn. quadr. 743 .

Quadrupes volatile ruffiae. ACt. petr. 5. p.218.

Habitat in borealibus Europae, Afiae, Americae. 



$$
\text { ri. Pecora } \alpha \cdot \eta^{35} \text {. }
$$

20. Cameluo. L.1735. 


\section{PECORA.}

Dentes Primores fuperiores mulli.

Pedes vngulati.

lifferiores VI aut VIII, a molaribus remotifinit.

Mammae inguinales.

28. CAMEL,VS, Cormua nulla.

Dentes Primores inferiores VI, fpathiformes.

Laniarii diftantes: fuperiores III, inferiores II.

Labium fuperius fiffum.

Drome- I. C. topho dotfi vnico. Syft. nat. 13. 12. I.

darius. Camelus unico in dorfo gibbo. Rai. quadr. 143.

Camelus. Ionft. quadr. t. 41. Gefin. quadr. 159. Aldr. bif. yo8.

Habitat in Africae defertis arenofis fiticulofis.

Callos in pedibus anterioribus 4 , in pollerioribus 2 , in Jlerno I. gerit. Ventriculus fecundus cellulofis pro aqua pura diutius ajseruanda per fiticuloja deferta. Onera portat, lente feflinat, la fus iacet fuper pectus.

BaAtria- 2. C. tophis dorli duobus. Syft. nat. 13. n. 2.

nus. Dromedarius. Ionft. quadr. I. 44. f. I.

Camelus. Gefir. quadr. 151. Aldr. bif. 907.

Habitat in Africa.

Curfu antecedente celerior, pro magnatibus, varior.

Glama. 3. C. dorfo laeui, topho pętorali. Syft. nat. 13. ́n. 3 .

Camelus peruuianus Glama dietus. Rai. quadr. 145 .

Pelon ichiatloquitli. Hern. mex. 660.

Habitat in America meridionali. Frigoris impatiens.

Cicuratur, onera portat, fatigata ficcumbit, compreffis tefficulis obflinatus compellitur; a vomitu boftem pollit, faepe Catocho obnoxia. 
Pacos.' 4. C. tophis nullis, corpore lanato. Syf.nat. I3. n. 4 . Camelus peruuianus laniger Pacos dicus. Rai. quadr. I47.

Hubitat in America meridionali.

Totus Janguineus, Jibutus albus; Pedes didnctyli. Priori minor, magis lanatus, nec oncribus portandis par.

29. MOSCHVS. Cormua nulla.

Dentes Laniarii fuperiores folitarii exferti.

mofchi- I. Mofchus. Sy?t nat. 13 .

ferus.

Anim.l mofchiferum. Rai. quadr. 127.

Capreolus mofchi. Gefn. quidr. 695 .

Capra molchus. Aldr. bifislc. 743. Ionft. quadr. 55 . t. 39 .

Mofchus. Sclbroeck. monogr.t. 44 .

Habitat in Tataria verfus Chinam.

Mufcbus fubftantia vnctuoja ambrofaca e folliculo prope vimbilicunn.

Scbroecki Hiftoria Mofchi. Wien. 1682. quart.

30. CERVVS. Cormua folida, tenera corio hirto tecta apiceque crefcentia, denudata, annua.

Dentes Primores inferiores VIII.

Lamiarii nulli (interdum folitarii fuperius).

Camelo- I. C. cornibus fimpliciffmis, pedibus anticis longiffimis. pardalis. Syjt. nat. I3. n. $\mathrm{r}$ :

Camelo-pardalis. Bellon. itin. Irg. Rai. quadr. 90. Haffelqu. act. Vpj. 1750. p.15. itin. 203.

Habitat in Aethiopia et Sennar.

Pronus cubat more Cameli; Gramen depafcitur diuaricatis pedibus anticis, fed arborun inprimis vefcitur foliis.

Álces. 2. C. cornibus acaulibus palnatis, caruncula gutturali. Syft. nat. 13. 17.2. Fr. Juec. 37 . 
29. Moschus L 174 s.

30 Cerver $23^{35 .}$

30a. Giraffa. Briso.1756. 

Alce. Gefin. quadr. I, 3, 4. Aldr. bifulc. 836. Ionft. quadr. t. 30. Bonan. muf. t. 295. Olear. muf.t.9. f: 2. Rai. quadr. 39 .

Habitat in borealibus Europae, Afiaeque Populetis.

Vngula ferit; 50 milliaria de die percurrit; corium globum plunbeum fere eludit.

Elaphus. 3. C. cornibus ramofis teretibus incuruis. Fatn. Juec. 38 . Syft. nat I3. n. 3 .

Ceruus. Gefn. quadr. I, 3, 4. Aldr. bifulc. 774. Ionft. quadr. t. 3I. Rai. quadr. 84 .

Habitat in Europa, Afia.

Aetas Bouis tontun; fabula eft longaeuitas cerui. Cyfide fellea caret Cerui genus.

Anatome. E. N. C. cent. 10. app. 448.

Taran- 4. C. cornibus ramofis teretibus: fummitatibus palmatis. dus. Fann. Juec. 39. Sy/t. nat. 13.n.4.

Rangifer. Geju. quadr. I4I. Olear. minf.16.t.Io. $f .3$. Rai. quadr. 88.

Tarandus. Aldr. bifulc. S59. t. 861.

Rheno. Amocn. acad. 4. p.

Habitat in Alpibus Europae ct Afiae maxime Septentrio. nalibus, vicfitat Licbone vangiferino.

Vterque fexus cornutus eft vt in Canelopardale, rano infino ctian palmato; cuftratus quotannis cornun deponit. Vellus denfiffrmum. Pulices, Oeftra, Tabani ad alpes cogunt, crepitantibus vngulis. Pecora Lapponunu cafeofa. Aetas XVI. annorum.

Anatome. Barth. att.1671. 12. 135.

Dama. 5. C. cornibus ramofis compreffis: fummitatibus palmatis. Iann. Suec. 40. Syft.nat.13.n. 5 .

Dama. Bell. itin. 57. Gejn. quadr. 84. Aldr. bifulc. 741. Ionft. quadr. 82.

Ceruus platyceros. Rai. quadr. 85 .

Habitat in Europa.

Arcetur filo borizontali.

Bezoat- 6. C. cornibus ramofis teretibus erectis: ramis tribus.t ticus. Mazama. Hern. mex. 324.

Caguacu-etc. Marcgr. Liraf. 235. Pif. braf. 98. Rai. quadr. 90.

Ilabitat in America aufrali. 
Capreo- 7. C. cornibus ramofis teretibus ereetis: fummitate bifida. lus. Faun. fuec. 41. Syjt. nat. 13. n. 6.

Capra, Capreolus, Dorcas. Gejin. quadr. 64. Ionft. quadr. 33. t. 33 .

Caprea plinii, Capreolus. Aldr.bifulc.738. Rai.quadr. 89.

Habitat in Europa, Afia.

guineen- 8. C. grifeus fubtus nigricans. Muf: Ad. Fr. I. p. 12.

fis. Habitat in Guinea.

Cormua $n 0$ ridi, tanucn buizs generis.

31. CAPRA. Cornua coneaua, furfum verfa, erecta, fubra. Dentes Primores inferiores VIII. Laniarii nulli.

Hircus, I. C. comibus carinatis arcuatis. Faun. fivec. 42.

Capra. Giefin. quadr. 242. Aldr. bifulc. 635. Ionft. quadr. 47. Rai. quadr: 77.

B. Capra angolenfis. Hafjelq. iter. 206.

Habitat in montofis, rictitans ex ramulis variis frondibusque arbornn, Liclsenibus.

Pecus barbatun, intidum, Jäliens, falax, bircum olens, decorticans; corio tenaci indutum, lana caprina teEftun, arunco barbatum, bellicofin, cafeofunn; Pedum cafiratio fingularis. Edit etian Cicutam.

C. Angolenfis Lana ad pedes vsque dependet niveä, Jericum prouocans camelina dicta.

Ibex. 2. C. cornibus nodofis in dorfum reclinatis. Syft."nat. I4. n. 6.

Ibex. Olear. muf. t. I0. f. I. Rai. quadr. 79. Wagu. beilu. 176.

Habitat in Wallefine praeruptis inacce/fis.

Cornibus vaftis reclinatis, corpore fulluo, arunco nigro.

Rupica- ว. C. cornibus erectis vncinatis. Syft. wat. 14. n.5.

pra. Rupicapra. Bell. itin เ26. Olear. muf t. 9. f 3. Gefin. quadr. 40. Aldr bifulc. 727. Dodart. act. 225. Scheuch. alp. 155. Wagn. belu. 183 .

Habitat in alpibus Helueticis funmis inaccel/fs. Ruffo-fufca, Sed alba fronte, vertice, gulda, auribus intus. 4. C. 
31. Capra 2.1935 (Bhirans Briss.1956.)

31. a! Traguluo Oriss. 1756. 

depreffa. 4. C. cornibus depreffis incuruis minimis cranio incumbentibus. Syft. nat. I4. n.2.

Habitat in America.

Magnitudo baedi Hirci. Pili longi, pendulli. Cornua lunata, craffa, vix digitum longa, cranio adpreffa vt fere cutem perforent.

reuerfa. 5. C. cornilus ereçtis apice recuruis. Syft. nat. I4. n. 3. Habitat in America.

Magnitudo baedi Hirci wnins anni. Pili breutes, ceruini. Cornua vix digitun longa, antrorfinn recuvuata apice. Haec cum praccedenti coibat, et pullum nonz diu Superftitem in viuario Cliffortiano producebat. Facies vtriusque adeo aliena, vt vix jpeciem eandem, at dinerfifimam argueret.

pygmea. 6. C. cornibus teretibus conicis obtufis curuis: latere incuruato rugofo.

Capra pedibus digito humano anguftioribus. Syft. nat. I4. 12. 4 .

Ceruus pufillus guineenfis. Seb. muf. I. p. Io. $t .43$. $f: 3$.

Habitat in Guinea, India.

Gazella. 7. C. cornibus teretibus reçiffimis longifimis bafi annulatıs. Syft.nat. I4.n. 8 .

Gazella indica, cornibus reetis longiffimis nigris prope capur tantum annulatis. Rai. quadr. 79.

Habitat in India.

Ceruica- 8. C. cornibus teretibus dimidiato-annulatis contortis. Syft. pra. nat. I4. n. 7 .

Gazella africana. Rai. quadr. 79 .

Capri-Cerua. Kacmplb. amoen. 398.t.407.f. r.

Hebitat in India, Alia.

Color Capreoli, ventre albo, cauda nigra.

bezoar orientale farpe in buius quarto ventriculo.

Dorcas. 9. C. cornibus teretibus perfecte annulatis contortis. Sy/l. nat. 14. n. 9 .

Gazelia africana, cornibus breuibus ab imo ad fummum fere annulatis, circa medium inflexis. Rai. quadr. 80.

Habitat in Africa. 
grimmia. Io. C. capite fafciculo tophofo, cauitate infra oculos. Syft. nat. I4. $n .10$.

Capra fylueftris africana grimmii. Rai. quadr.80. Habitat in Africa.

mambri- II. C. auribus pendulis longiffimis. Syft. nat.I4.

ca. Capra mambrica f. fyriaca. Gefn. quadr. 153. Rui. qusodr. 81. Aip. aegypt. 229.

Habitat in Irdia.

Ammon. I2. C. cornibus arcuatis, collo fubtus barbato, cauda nigra.t

Tracelaphus et Mufimon. Gefi. quadr. I55.

Habiant in Sibiria. Gnelin.

Medium quafi inter Capras et Oues.

Aninialis buius biftoria dejideratur.

32. OVIS. Cormitit côncáita, retrorfum verfa, intorta, rugofa.

Dentes Primores inferiores VIII.

Laziariii nulli.

Aries. I. O. cornibus compreffis lunatis. Faun. fuec. 43. Syft. nat. I5. n. I.

Ouis. Gefin. quadr. 138. Aldr. bifulc.370. Ionft. quadr. t. 22. f.1.2. 3. Rai. quadr. 73 .

ruftica. $\alpha_{0}$ cormuta.

anglica. B. mutica, cauda frrotoque ad gemua pendulis.

bijpanica. $\gamma$. cornuta, cormun Spira extrorfam tracta.

polycerat. ¿. Polycerata e Gotlandia 248. Aldr. bifulc. 397.

afiricana. E. africana. Rai. 75. pra lana pilis breuibus birta.

laticau- S. platyura arabica.

data.

Habitat in ficcis apricis calidis.

Ouis cum Ariete Verucce Agnoque edit imprinis $\mathrm{Fe}$. ftucan ouinam, in aruis Burfam paftoris, laeditur a Pruno fpinofa, Equifeto, Flannunla, Antberico, Kalnia, Myofotide aquatica, Anemone nemorosa. Parun bibit, ab aqua ftagnante vermes Duodeni Hepatisque; praecautur muria; mutat aetate dentes; fert Lanam; laetificat pafcua; timida pellit boftem calcitratione, vrina. Mas vnus fufficit 20 feminis, grasidis 150 diebus. Opilio pera pedoque inftructus et cane, c caula minus clanfa educit balantes greges in cam- 
32. Orio. 20y35. (Aries Buis. 1756.) 
33 Boo. L1.735. 
pos fiticulofos, apricos. Infeftatur Hipoobofca, Pediculo, Oefiro, Acaro. Laborat Vertigine, Hydrope, Pbtbifi, Iitero, Hepatitide.

guineen- 2. O. auribus pendulis, palearibus laxis, occipite prominenfis. te. Sy/t. nat. I5. n. 3.

Aries guineenfis f. angolenfis. Marcgr. braf. 264. Rai. quadr. 75 .

Habitat in Guinea.

Cornua parua, deorfum rqque ad oculos torta. Iuba fisb collo pendula. Pili breues, bircini.

Streph- 3. O. cornibus rectis fpiralibus. Syft. nat.15.n.2.t ceros. Strepficeros cretenfis. Bellon. itim. 20. Aldr. bifulc. 407. Rai. quadr. 75 .

Habitut in Ida monte.

33. BOS. Cormua concaua,- antrorfum verfa, lunata, laeuia.

\section{Dentes Primores inferiores VIII. Laniarii nulli.}

Tauris. I. B. comibus teretibus extrorfum caruatis, palearibus la. xis. Fann. Jilec. 44. Syft. mat. 15. 11. I.

B. Bos. Gefrn. quadr. 25. Aldr. bijullc. 13. t. 36. Ionft. quadr.t. 14. Rai. quadr. 70.

a. Vrus. Caefar bell. gall. VI. c. 5 .

Habitat in Poloniae deprefis graminofis ferus Vrus. Mugiens boansque Bos: Tauro, Vacca, Vitulo, bomin: vtili/frnus pro agris lactificandis, colendis fub iugo: pollet carne, lacte, cafeo, butyro, febo, corio; infeftatur Oeftris, Tabanis, Cynipe, Ricino, Pediculis; laeditur a Cicuta, Aconito, Anemone. Vitulus tendinofus brenioris atui fingularis.

Bonafus. 2. B. cornibus in fe flexis, iuba longiffima. Syft. nat. 15. n. 2 .

Bonafus, Rai. quadr. 7I. Ionft. quadr. t. 1S. f. I.

Habitat in Africa, Afia.

Defendit fe feercore, ad aliquod palfus deiedo, combureate. 
Bifon. 3. B. comibus diuaricatis, iuba longifima, dorfo gibbofo. Syft. nat. $15 \cdot n \cdot 3$.

Bifon. Rai. quadr. 7I. Geji. quadr. 296. Ionft. quadr. $t .17 . f .2$.

Bifon americanus. Catesb. carol. 3.p. 20.

Taurus mexicanus. Hern. mex. 587. Gejn. quadr. 30I.

Habitat in Mexico, Florida.

Collum et Pectus vndique vrfi inftar birfuta.

Bubalis. 4. B. cornibus refupinatis intortis antice planis. Syft, nat. 15. 11.4 .

Bubalus. Rai. quadr. 72. Geen. quadr. 58. Aldr. bijillc. l. I. c. 6.

Habitat in Afia. cultus in Italia.

Lana duriufcula in dorfo, caeterum pili ravi. Regitur anmulo narium; trabit currus; oryzam colit; cafeum di canallo praebet.

indicus. 5. B. cornibus aure breuioribus, dorfo gibbo, iuba nulla. $t$ Bubalus paruus indicus. Edwo. all.200. t. 200.

Habitat in China.

Vtrums baec jpecies diftincta a praecedentibus, nec ne, dubito. 

VII. fumenta Liy3s (Belluae L. vos.)

34 Equess Lे/735 


\section{BELLVAE.}

\section{Dentes Primores obtue truncati.}

Mammae duae inguinales.

34. EQVVS. Dentes Primores fuperiores VI erecto - paralleli. Inferiores VI prominentiorcs.

Laniavii folitarii, inclufi, vtrinque remoti.

Pedes vngula indiuifa.

Caballus. I. E. cauda vndique fetofa. Faum. Suec.34. Syft. nat. Ir. Equus. Gefin. quadr.132. Aldr. vngu. 12. Ionft. quadr. I. t. I. 2. 3. 4. Rai. quadr. 62.

Habitat in Europa.

Animal generofinn, fuperbum, fortiffinum in currendo, portando, trabendo, aptifimunn equitando, curful fusvens; Jyluis delectatur; pofteriora curat; cauda mufars abigit; alterum fcalpit; pullum iniuriae obnoxium reponit; binnitu focium vocat; dormit poft noEtem; calcitrando pugnat; fitdans fe volutat; vegetabilia edit boue propius, femina diffeminat; flercus incalcfoit; cyftide fellea caret; non vomit; Equuleus Hipponane natus, pedibus clongatis; laeditur globulo auris, acicula pedis, nafi capiftro, dentibus fibo illitis, Padi berba, Pbellandrii Curculione. Laborat bernia mediaftini, polypo cordis, ortbopnoca, oeftro faucium anique, fcabie, tavtaroque pedum, Bubone colli; Hippocomia inftruitur.

Afinus. 2. E. cauda extremitate ferofa, cruce nigra fupra humeros. Faun. Since. 35. Sy/t. nat. II.

Afinus. Gefin. quadr. 40. Aldr. vng. 295. Ionft. quadr. 12. t. 6. Rai. quadr. 63.

Onager. Rai. quadr. 63. fylueftris eft.

B. Mulus. Rai. quadr. 64. ab Equa et Afino. \%. Hinnus. Rai. quadr. 64. ab Ajina et Equo,

Habio

Solidungula mascula mammas non habent. Ariffot. 
Habitat in oriente, frigoris impatiens.

Visfitat Jpinis; tardus, flupidus, rudens, libidinofiss; auriculae magnae.

Zebra. 3. E. fafciis fufcis verficolor. Syft. nat. Ir.

Zebra. Edwo. au. tab. dina vltinue. Rai. quadr. 69. Ionft. quadr. t. 5. f. I. Aldr. quadr. l. I. c. g.

Habitat in India, Africa.

Color albidus fou rufe fcens fafciis nigris digitos tres la. tis per totuin corpus tronsuerjis. Cauda Afmi.

35. HIPPOPOTAMVS. Dentes Primores fuperiores VI, per paria remoti. Inferiores IV prominentes : intermediis recta protenfis.

Laniarii folitarii, oblique truncati. Pedes margine vnguiculati.

amphi- $\quad$ 1. H. pedibus quadrilobis.

bius. Hippopotamus. Bell. aquat. 28.t. 30. Grew. muf.t. r. f. 5. Alp. aegypt. 245. t. 22, 23, 24, 25. Matth. diofic. 2.c. 22. Rai. quadr. 123. Ionfli. quadr.t. 49. Syjt.nat. II.

Habitat in Nilo et Bambolo Africae et all oftia funiorum Aliae, ampbibius.

Corpus Suis, nudum praeter vibriffas, magnitudine $V_{r} \cdot i_{\text {, }}$ Pedibus brenibus, Mandibula fuperiore mubili; dentibus fcintillans. Mammae $\mathbf{I}, \mathbf{x}$, inguinales; incedit nec natat; bumiles aggeres vix adjcendit; pabulatur in fundo et ficco; Oryzae et Colocafiae dasnnumb adfert. Lupino pellitur. Eebemot lobi.

terreftris. 2. H. pedibus pofticis trifulcis. $\dagger$

Tapirierete. Marcgr.braf. 229. Rai. quadr. 126.

Habitat in Brafilia.

Animal dubium, Hippopotamo genere proximunn, prae: esste Roio. 
35 thippopotamesos 21735

- 351. Eppino 3iso. 756 


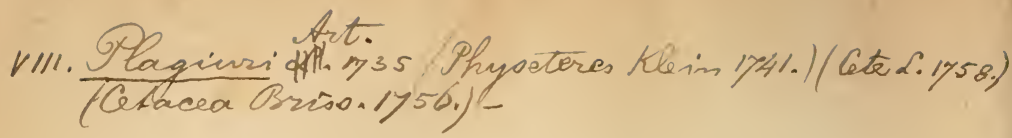

A. Fan. Honsodontidae.

36. Wonvon Ait. 1735 (Bratodon Brisu.eyst.)

B Farn. Balaenae. Klein 1yul.

37. Balaena Art 1935 . 


\section{C E T E.}

Spivacula fupra Caput.

Pinnae Pectorales candalisque borizontalis - ausque vnguibus.

36. MONODON. Dens in maxilla fuperiore exfertus, praelongus, rectus.

Fiffula in vertice.

Mono- I. MONODON. Art. gen. 78. Jyn. 108. Faun. Jitec.263. ceros. Syft. nat. 39 .

Monoceros pifcis. Will. icbt. 42. Rai. pifc. II.

Vnicornu marinum. Tulp. obf: 375 .

Habitat in Oceano Septentrionali Americae, Europae.

Cormu Monocerotis jpirale buius Dens eft.

37. BALAENA. Dentium loco in maxilla fuperiore laminae corneae.

Fiftula duplex.

Myftice- I. B. naribus flexuofis in medio capite, dorfo impenni. Avt. tus. Balaena Will. icbt. 35 .

Balaena vulgo f. Mufculus. Rond. pifc. 475 .

Balaena vulgo f. Miyfticetus arifotelis. Gieji. $p: \int c$. Ir4. Balaena vulgaris edentula, dorfo non pinnato. Rai. pilč. 6 . Balaena fpitsbergenfis. Mar. Jpitsb. 98. t. Q. f. A. B.C. Habitat in Ocermo Groenlandico.

Maximus omnizm animalitm, faspe 100 pedum, viffitat medufis. Caput grande; Dorfum impenne.

Fiftula mulle, fed 2 foramina fiex:orfa.

Mammae abduminales ante vuluan; Maxillofi Dentes flexiles fifilesque viunles. Pinguedo copiofiffima, vt ex vnico faepe uneretur nauis.

Phyfa- 2. B. naribus in medio capite, dorfo extremo pinna adilus. pola. Ait. gen. 77. Jyn. 107. Faun. Suec. 265. Syft. nat. 39. 2.

Balae.

Cetis quibusdam pinna dorfalis, omnibus pinua caxdae et pedtorales; nulli i r. ani, ant ventrales, $\mathrm{cnm}$ pedes in candam coadunati, 
Balaena edentula, corpore ftrictiore, dorfo pinnato. Rai. pifc. 9.

Phyfalis bellua f. Phyfeter. Gejin. pifc. 723 .

Phyfeter. Rond. pifc. 485. Will. icht. 4I.

Finfifch. Martens. Jpitsb. 125. t. Q. f. C.

Habitat in Oceano Europaeo.

hoops. 3. B. fiftula duplici in roftro, dorfo extremo protuberantia cornea. Art. gen. 77. Jym. 107. Muf. Ad. Frid. I. p. 50.

Balaena tripinnis nares habens cum roftro acuto et plicis in ventre. Rai. pifc. 16.

Habitat in Oceano Septentrionali.

Mufcu- 4. B. fiftula duplici in fronte, maxilla inferiore multo lalus. $\quad$ tiore. Art. gen. 78. Jjm. 107. Syft. nat. 39. n. 3. t Balaena tripinnis, maxillam inferiorem rotundam et fuperiore multo latiorem habens. Rai. pifc. 17 .

Habitat in mari Scotico.

Modo bic a Myfticeto fufficienter diftinctus; conferatur Muf. Ad. Fr. 51.

38. PHYSETER. Dentes in maxilla inferiore. 'Fufula in capite $f$. fronte.

Catodon. I. P. dorfo impenni, fiftula in toftro

Catodon fiftula in roftro. Art. gen. 78. Jyn. 108. Syft. nat. 39. n. I.

Balaena minor in inferiore maxilla tantum dentata, fine penna aut fpina in dorfo. Rai. pifc. 15 .

Habitat in Oceano Jeptentrionali.

macroce- 2. P. dorfo impenni, fiftula in ceruice.

phalus. Catodon fiftula in ceruice. . Art. gen. 78. Jyn. 108. Fauin. fuec. 262. Syft. nat. 39. n. 2.

Cere. Cluf exot. I3I.

Cere Clutio defcriprum. Will. icht. 4I.

Balaena maior in inferiore tantum maxilla dentata ma* crocephala bipinnis. Rai. pijc. 15 : II.

Habitat in Oceano Europaeo.

Longitudo faepe essxaginta pedum.

Sperma Ceti e ventriculis cerebri.

microps. 3. P. dorfo fpina longa, maxilla fuperiore longiore. Art. gen. 74. Syn. 104. Syft. nat. 39. n. I. 
30 . Phypeter Ast.1738.

305. Catodon. Drtioly35:-(Cetus ussion 1yst)

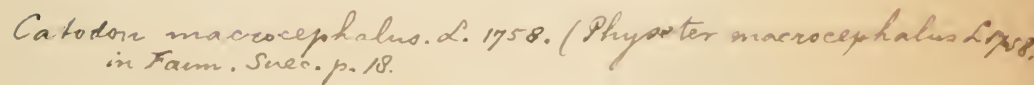


C. Fam. Delphaces Nein 1941. (Porcelli Hein 174)) 39 Delphimus Ast. 1y35.

1. Delphimus phocoena C. 1yso. (Aelphimus phocaena hilybir) 
Balaena maior inferiore tantum maxilla dentata, dentibus arcuatis falciformibus, pinnam if fpinam in dorfo habens. Rai. pifc. I5.

Habitat in Oceano feptentrionali.

Turfio. 4. B. dorfi pinna altiffima, apice dentium plano. Art.gen. 74. Jyn. 104. Sy/t.nat.39. n. 2.

Balaena macrocephala tripinnis, quae in mandibula inferiore dentes habet minus inflexos et in planum definentes. Rai. pifc. 16.

Habitat in Oceano Jeptentrionali.

4

39. DELPHINVS. Dentes in maxilla vtraque. Fizfula in capite.

Phơcoe- 1. D. corpore fubconiformi, dorfo lato, roftro fubobtuna. fo. Art. gen. 75. Jyn. 104. Fain. Juec. 266. Syft. nat. 39. n. I.

Phocoena. f. Turfio. Bell. pifc.16. Gefin. pifc.7II.

Phocoena. Rond. pifc. 473. Will. icbt. 31. Rai. pifc. 13. Habitat in Oceano Europaeo et Balthico.

Delphis. 2. D. corpore oblongo fubtereti, roftro attenuato acuto. Art. gen.76. Jyn 105. Syft. nat. 39. n. 2.

Delphinus. Bell. pijc. 9. Rond. pijc. 459 .

Delphinus antiquorum. Rai. pijc. 12. Will. pifc.28. Habitat in Oceano Europaeo.

Orca. 3. D. roftro furfum repando, dentibus latis ferratis. Art. gen. 76. fyn. 106. Faun. fuec. 267. Syft. nat. 39. n. 3. Orca. Bell. pifc. 18. Rond. pifc. 483. Giefn. pifc. 635. Will. iclbt. 40.

Balaena minor, vtraque maxilla dentata. Rai. pifc. 15. Habitat in Oceano Luropaeo.

\section{CLAS}

SIREN Barth. cent. 2. obf. 12. 2. 188. E Brafilia Cultro anatomico Leydae Jubiecta, mibi paradoxa ob Aures exffar:tes, Collumque anguftum in aquatzso Mammali, nec a peregrinatoribss vlterixs confirmatc. 


\section{CLASSISII.}

\section{A V E E S.}

AEREAE vocales Volucres pulcherrimae, Mandibulis protract is mudis, Corpore pennis imbricaro, Alis duabus pennatis volitantes bipedes dignofcuntur.

A VES leuiffimae modicae magnitudinis, tectae pennis plumisque, deftitutae Auriculis, Labiis, Cauda, Scroto, Dentibus, Vtero, Epiglottide, Fornice, Corpore callofo, Diaphragmate.

PENN AE auibus propriae imbricatim in quincuncem difpofitae, in • terftinftae Plumis, diftinetae a Remigibus Rectricibusque, omnes, e bafi cylindró concauo vatis lymphaticis fimili, elongatae bifariam radiis parallelis approximatis diftinetis itidem pinnatis.

Colore ludunt, fed variant aetate, fexu, tempeftate; conftantiores Remiges et Rectrices, quae attente notandae. $\left({ }^{*}\right)$

OVA his, animalibus communia (pag. 9. n. 3.), numero diverfis vario, fed maiora pro mora exclufionis ficcae, femper putamine calcario obtecta, incubatu calido excludenda plerumque Parentis.

DIVIDIT V R Auium corpus in Caput, Truncunn, Artus.

CAPVT impofitum Collo elongato, erętiufculo, teretiufculo. Mandibulae nudae, edentulae, exporrectae in Roftrum corneum, Naribus pertufum, bafi quibusdam Cera nuda veftitum, Vibrifis pectinatim digeftis ad faucis exterioris margines.

Lingua carnofa, cartilaginea, emarginata, lacera, ciliata, integra $f$. acuta.

Oculi laterales palpebris, Membranaque nicititante. Aures truncatae absque auriculis.

Ornamenta verticis e Crifta pennacea $f$. Caruncula frontis gulaeue.

TRVN.

f*) Ait. Stockh. 2740. p. 370. t.1. f.20, 21. 
Ares. L.1\%35: 

TRVNCVS ouatus conftans Dorfi coadunato macro, fubtus Sterno magno carinato mufculofifimo, Claniculis coadunatis in Furcam, Abdomine minore poftice fub vropygio perforato ano, genitalibus communi.

A R T V S comprehendunt Alas, Candum, Pedes.

ALAE 2 ex Brachio et Cubito manibus priuato conftant, quae poftice fornicato-ciliarae Pennis Rennigibus: exterioribus Primoribus Cubiti, pofterioribus Secundariis brachii, ala notba fupplente rudimentum Pollicis. Hae remiges proxime teguntur Tectricibus alarum proximis et vltra.

CAvDA ex Vropygio cordato, gemina glandula foeto, fupra pertufo, poro penicillo cincto, ambitu ciliato Pennis Rectricibus faepius 12: pede longioribus in Macrouris, breuioribus in Brachytris, funtque vel aequales (C. integra), vel ad latera breviores (cuneata), vel ad latera longiores, (forficata).

PEDES 2, ex Femore, Tibia, Digitis et Vnguibus.

Femora carnofa, pennis teata, fed feminuda in Grallis.

Tibiae tendinofae, plerisque nudae, anguftae, rarius hirfutae, paucis Calcari poftice cornutae.

Digitis pedes infiftentes faepius 4 , anterioribus plerisque tribus, pollice vnico poftico, nifi in Scandentibus digitis anticis duobus totidemque pofticis, yel curforiis absque poftico. Fiffi funt et diftincti Digiti in plerisque terreftribus (Fiffipedes); Jemipalmati in paluftribus; membranis vel Lobati, aut membrana toti Palmati (Palmipedes) in natantibus.

Vnguibus inftruuntur Digiti plerumque, rarius pofticus abs. que articulo eft.

VOLA TILI A haec, Telluris vigiles libidinofique mufici, plerumque inermia funt, cum Alarum remigibus te facile in aethera recipiant ab holtibus terreftribus, et Caudae reetricibus aëreos diuerfo volatu eludant, nifi in terra negotiari neceffo fit, dum odor vel ingratus hoffes arcet, vel abolitus occultat; dum aliae fpeculantur hoftemque appropinquantem indicantque aliis.

ARMA paucis: Cornua Meleagridi cornutae, Anhimae.

Calcariu Paunni, Phatiano.

Spinae (axillires) Strurhion, Camelo, Fulicae fpinofae, Charadrio fpinofo, Anhimae. 
VENERE monogama iunguntur pleraeque Aues, pclygana panciores, vt Gallinae, Anates. Illae vtriusque fexus coniundtis viribus ftruunt nidum faepe artificiofifimum, vteri fuccedaneum, incubant fedulae ouis, pulios famelicae alunt, fouent, curant, pro aris et focis defendunt $\left({ }^{*}\right)$, dum in his otientur mares.

MIGRATIO NES annuas inter feptentrionales aeftiuas et aufrales hybernas plagas arcticae Telluris inftituunt non paucae, ne hyeme demergerentur boreales omnes apud nos infectiuorae; fic migrant plurimi Paferes, inprimis tenuiroftres ob defectum infectorum; Grallae ob denegatum glacie aditum vermibus et amphibiis; Anferes ob congelatas aquas includentes pifces etc. Reuocant vero easdem dies aeftatis longiores, ciborum copia, denfiores veftitus, nuptiae paratae.

OPERA nec fine fructu peragunt fua fingulae: fedentes Accipitres cadauera auferunt, vt viuis parcant; incedentes Picae quisquilias impuras verminofasque deuorant; natantes Anferes aquarum nimios hofpites minuunt; vadantes Grallae inquirunt in paludum animalcula; currentes Gallinae decidua Grana legunt; falientes Paferes arbuftarum herbarumque femina infectaque carpunt; eoque varia femina Pifcium, Infectorum Plantarumque faepius in remotiffima loca ferunt. Conquiruntur venatu, Falconaria; Ancupio inftituuntur Falcones ad venatum, Diomedeae ad pifcaturam, Pafferes ad Muficam. Manfuefcunt Gallinae, Anates, Pafferesque varii: in ornithone $f$. Auiario coluntur ob carnes, oua, plumns, pennas, pulchritudinem, fonum. Afferuantur exuluiae, ficcatae, $\int$ piritu contentae.

ORNITHOLOGI eminent inter veteres Bellonius, Gefizerus, Aldroundus; inter recentiores Willugbbaeus; inter recentiffimos Marjigli, Albinus, Seba, Catesbaeus, eximiusque Edwardus. Syftematici Raius, Barrere, Kleinius, Moebringius. 

I. Accipitres L.y3.5

II Picas $\alpha .1735$

Itr Anseres. 2.1735:- 


\section{ORDINVM Characteres.}

\section{A C CIPITRES.}

rostrvm (Vncus trabens) fubincuruatum: Mandibula fuperiore pone apicem vtrinque dente armata: Naribus patulis.

PEDES infidentes, breues, robufti: Digitis fub geniculis verrucolis; Vnguibus arcuatis, acutiffimis.

corpvs Capite Colloque mufculolis; Cute tenaci. Impurum.

V I C T V S: Laniena rapinaque e cadaueribus.

NıDvs in altis; Oua circiter 4; Femina praeftantior; Monogamia.

\section{PICAE.}

R OSTR V M (Cuneus farriens) cultratum dorfo conuexo. PEDES ambulantes, breues, validiufculi.

CORPVS tenaciufculum, impurum.

VICTVS e Quisquiliis.

NIDvs in Arboribus; Mas alens feminam incubantem; Monogamia.

III. ANSERES.

ROSTRVM (Cribrum colans) laeue, epidermide tectum, apice auktum.

PEDES natatorii digitis membrana palmatis; Tibiis comprefis, breuibus.

corpvs pingue, Cute tenaci. Plumis praeftantioribus. Rancefcens.

v 1 crvs in Aquis ex plantis, pifcibus, etc.

NIDVS faepius terreftris; Mater non porrigit pullis; frequentius Polygamia. 
IV. GRALLAE.

ROSTRVM (Bacillus tentans) fubeylindricum.

PEDES vadantes Femoribus feminudis.

Corpvs compreffum pelle tenuiffima; Cauda breui. Sapidum.

viCTvs in Paludibus ex Animalculis.

NIDvs in terra faepius. Nuptiis variis.

\section{GALLINAE.}

roSTRVM (Harpa colligens) conuexum: Mandibula fuperiore fornicata fupra inferiorem; Nuribus membrana cartilaginea fornicatis.

PEDES curforii Digitis fubtus fcabris.

CORpvs febaceum, mufculofum. Purum.

viCTVs e Granis in ingluuie macerandis. Puluetatrices. NIDvs in Terra absque arte. Ouis numerofis. Pullis. cibus demonftrandus. Polygamia.

VI. PASSERES.

ROSTRVM (Forceps excipiens) conico-acuminatum.

PEDES falientes, teneri, fiffi.

Corpvs tenellum: Graniuoris Purum. Infectiuoris Impurum.

v I C T v $S$ e Seminibus aut Infectis,

NIDvS artificiofus; Pullis cibus inculcandus. Monogamia. 
III.a. Wacrorhymchae Ri.1y35. - IV. Sculopaces R.1y35.-(Grallar 2.1750)

T. Gallinae Lin3s:

II. Passeres. A. y35.- 
I. Aecipitres, L.1935

\$0. Vuetur. Klein 1950.-

41. Fales C. 1735. (Accipiter Klein 1750) (Aquila

42. Klein $1750 .-$

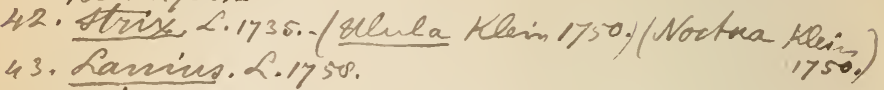

43 . Ampelis. 2.1735 .

II Picas R.1735.

44. Pittacus. L1735.

45. Ramphastos C. 1948. (Nasutus Klein 1950).)(PP." perivarns Klein 1750.)

46. Buceros. 2.1948.

4\%. Crotophaga Kiy"0.

40 Cowns 2.n35- (Pica Klein 1750.)

$4 \mathrm{~g}$ Coracias 21735.

so Gracula. Lisso.

57, Paradiaca L.1735 (Par Rioex Gron.1756.)

52 Cuculuo L.1135

52 a. Tourace Klim 1950. (Tauracentlin 1250.)

537 Jymx L1748.

54. Piend. L 1735.

55 titta 2.1735.

6 Ispida A. 1735 (theopon Hlem 1450.

57 theropes Hein 1750

Upipa. L. 1735.

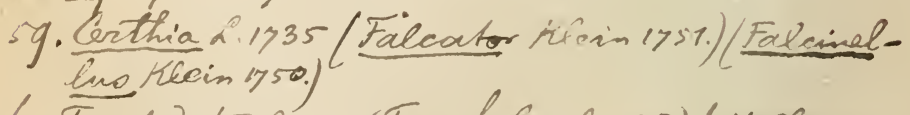

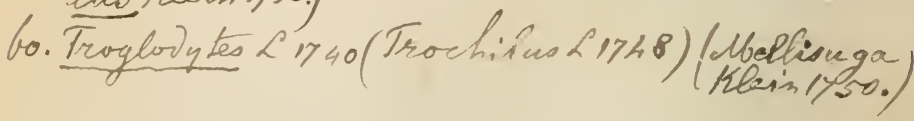




\section{CHARACTERES AVIVM.}

\section{ACCIPITRES.}

40. VVLTVR Roftrum aduncumm. Caput denudatumn.

4I. FALCO Roftrum aduncum baji Cera tectum.

42. STRIX Roftrum aduncum bafi Setis tectum.

43. LANIVS Roltrum rectiufculunz.

\section{PICAE.}

* Digitis anticis 2 , pofticis 2 .

44. PSIтTACVS Lingua carnofa. Mandibula fuperior adunca.

45. RAMPHAStos Lingua pennacer. Mandibula fiperior inanis.

-52. CVCVL S Lingua cartilaginea. Mandibula fiperior naribus marginatis.

53. I Y NX Lingua lambriciformis. Mandibula fuperior teretiufcula.

154. P I C v L Lingua lumbriciformis. Mandibula fuperior prifmatica.

* Digitis anticis 3 poltico I. Lingua incifa.

4S. CORvvS Mandibula fuperior cultrata bafi fetis ted $a$.

49. C OR A C I AS Mandibula fuperior cultrata bafi muda.

55. SITTA Roftum porrectum, rectum.

57. MEROPS ROftrum arcuatum, carinatum.

60. TROCHIL VS Roftum filiforme, capite longisu.

- Digitis anticis 3. pofico 1, Lingua integra.

46. B V C E \& O S Mandibula fuperior fronte offeo-gibbofa.

47. CвоторнаGA Mandibula fuperior compreffa, infracta, transuerfe fulcatie.

50. GRACVLA Mandibula fupervor comprefle, laeuis, bafe muda.

51. PARADISEA Mandibula fuperior ad medium v'sque plumofobolofericea.

- 56. Ax.CEDO Mandibula fuperior angulata. Lingua breuiffima.

58. V P $\vee$ A Rofrum arcuatum. Lingua obtufa.

59. CERTH1A Roftrum arcuatum. Lingua achta. 


\section{II. A NSERES.}

* Roftro apice vnguiculato.

61. A NAS Nares ounles. Dentes lanueliofi.

62. MERGVS Nares ouales. Dentes fubuluti.

64. PROCELLARIA Nares in cylindrum connatae.

65. DIOMEDEA Nares fubcylindricue, difiantes.

66. PELECANvS Nares lineares. Facies muda. Pedes Digitis ommibus palmatis.

* Roftro apice fimplici.

67. PHAETHON Roftum cultrato-fubulatum, fauce ampliore. Pedes Digitis 4 anticis.

63. ALCA Roftrum transuerje fafciatum. Pedes absque pofico.

68. COLymbvs Roftrum fubulatun, capite longius. Pedes pone aequilibrium.

69 LARVS Roftrum fibtus pone apicem gibbunn.

7O. STERNA Roftrum fubulatum.

?I. RHYNCOBS Roltri maxilla juperior breuior.

\section{GRALLAE.}

- Roftro magno capite longiore.

72. PHOENICOPTERVS Roftrum infractum, denticulatum. Pedes palmati.

73. PL A T A L E A Roftrum deprefun: apice rotundato-dilatato.

74. MYCTERI A Roftrum mandibulis adjcendentibus.

75. TANTALVS Roltrum arcuatum facco gulari.

76. ARDEA Roftrum rectum, acutiufculum.

80. RECVRVIROSTRA Roftrum fiubulato - attenuatum, recurแกนm.

77. SCOLOPAX Roftrum rectum, teretiufculum, obtufiufculum.

* Roftro capite vix longiore. Pedibus tetrada\&ylis.

78. TRINGA Roftrum teretiufculum, obtufun. Pollex vix infiftens.

82. FVLICA Roftri bafis fronte calua.

83. RALLVS Roftrum fubcavinatum. Corpus compreffufculum.

-84. DSOPHIA Roftrum fubfornicatum. Nares ouatae. 
III. Anderes. Liv3s:-

72 . Phoeni-copterus L. 1y4 o.

Gqa. Gymo 2.1935.

6r. Onas. Leys5. (Anoer Mlem 1750)

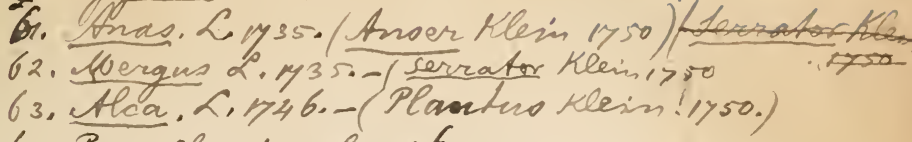

64. Procellaria 2.1746. -

65. Diomedea L 19s8.

66, Pelecanus 2.M.35. (Plawcens, Hletn v50.

66 a. Graenhus 2.1y35

6y. Phacthrocor. L. ns

68. Colymbus 2.1735:-

bo. Rarne $2 \cdot 1$ 1ys

70. Dherma R. ry 6 .

7 Rhymapd. 21y50.

III. A. Uacroshynchae L.M35.

73. Platelea L.1"35. (Platea L."141)(Dlatalea L.1350)

74 1tycteria Riys?

Ys Tounaluo Hein nso (Aoculator Klem nso,)

75.a. Kaculator Ruein 1y50.

Y6.b. Gruo. 21735 . -

76. Atdea 21735 (Kleim 1y50.peannita)

76 a. Ciconia R.1ys5 (Bbarniota Kteinn 1y>0)

IV. Lcolipaces. 2.1935. (Frallar. 2.1750.)

77. Scorlopax Niem 1yso. (Slareota Nerm ry50)

rya. Vurncmino है 1ysi.-

79. Tinga L. 1785

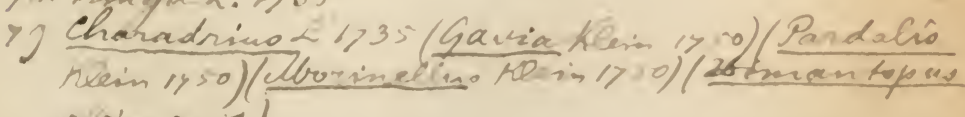
teien 1yso) 
80 Recurvinostra. 21746.

81. Hearmatopens a 1735. (Ootralega Klein 1y50)

01. a. Ortygometra א.1746.-

82. Fulica L.1735

03. Rallus Klein is 50.

04. Psophia. Liysos.

$04 a$. Vahellno L.11ys.

T. Gallinae.

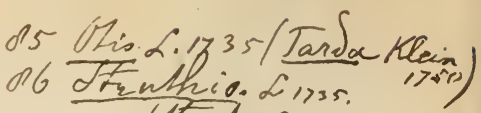

(Ptrutiox 1748 .)

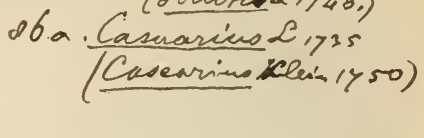

o7. PavoL1ys5.- Mamida L.1764. d6.A.F.p.ll.

So. Abeleagris 2.1335

og. Crax $20 y 4$.

go Gallina L 1735 (Gallus L1y40) (Gallinacens Klein "pso)( Hlector Kelein 1y50)

90. Phavianus \& 1740 .

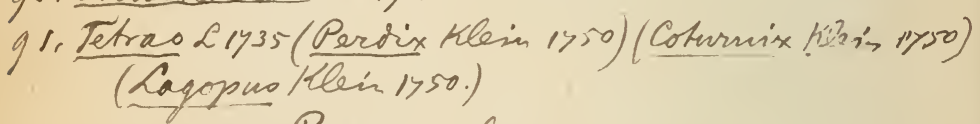

VI. Pasperes 2173 .

92. Columba Liyar (Columbus Klein cyso.)

93. Hlawa 21735 Tanagra 2.1764.M.A. F. past.

94. Thurmo L.1735. Pipra L. 1764. M.A. . F.p.11.

95 . Twrdun 2.1735 . Coceothraustes Mlim)

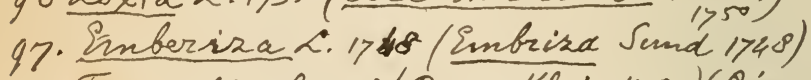

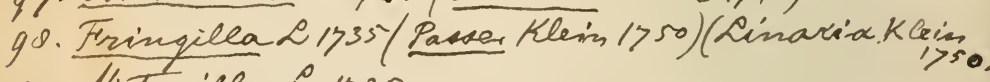

gg. Wotacilla 2. U35.-

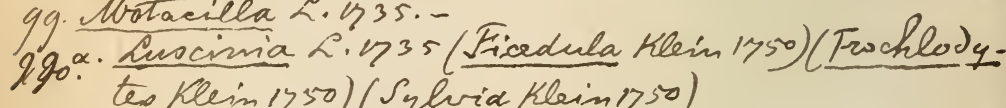
tenklein 1350) (Sylvia Kleinn50)

100 . Parus 21735

cos. Zbirundo L.1735

102-Caprimulgead 2. 1958. - 
- Roftro capite vix longiore. Pedibus tridactylis.

8I. HA EMATOPVS Roftrum compreffufculam apice cunco.

79. CHARADRIVS Roftum teretiufculum, obtufunt.

85. OT1S Roftrum fubfornicatum. Lingua emarginata.

86. Strvthio Roftrum conicum. Alae non volitantes.

\section{GALLINAE.}

87. PA v O Vertex pennis rectolutis. Roffrum mudun.

88. MELEAGR IS Facies carunculis verrucofa.

89. CRAX Bafis rofti Cera obtećta.

90. PHASIAN v Genae mudae, laeues.

9I. TETRA O Supercilia muda, papillojă.

\section{PASSERES.}

* Graminiuori crafiroftres.

92. COLvmBa Rofrum cera obliterata naribus tumidis.

93. ALAVDA Roftrum porreifum. Vnguis pollicis longiffe mus.

95. TVRDVS Roftrum naribus membrana femitectis.

96. LoX1A Roftrum conico-gibbum. Lingua intcgra.

97. EMBERIZA Roftrum mandibula inferiore latiore, lateribus Jirrfunt coaritiata.

98. PRINGILLA Roftrum conicum, rectum, aequale.

* Infectiuori tenuiroftres.

94. ' ŚTrR Nvs Roftrum mandibula fuperiore planiufcula andique marginata.

99. мотасіL A Roftrum fubulatum. Lingua lacera.

100. PARVS Roftrum fubulatum. Lingua truncita, quadrifeta.

10I. HIRVNDo Roftrum depreffum, incuruatum. Faux imsberbis.

102. CAPRIMVLGUS Roftrum depreffum, incurüatum. Faux vibrifis ciliata. 


\section{ACCIPITRES.}

Roftrum e Mandibula fuperiore denticulum vtrinque exferens.

40. VVLTVR. Roftrum tectum, apice aduncum: Caput impenne, antice nuda cute. Lingua bifida. -

Gryphus. I. V. maximus, caruncula verticali longitudine capitis. $f$

Vultur Gryps Gryphus. Klein. an. 45.

Cuncur. Rai. aul. II.

Habitare fertur Chili.

Ratra auis in terris; mibi ignota; videfis Kleinium.

Harpyia. 2. V. occipite fubcriftato. $\dagger$

Yzquauhtli. Hern. mex. p. 34.

Aquilae criftatae genus. Rai. au. 16r.

Habitat in Mexico.

Magnitudo arietis. Collum, Dorfum, Cauda, Crifa furrecta nigra; Subtus nigro candidoque filluo mixta. Homines etiam adoritur. Hernand.

Cfr. Aquila mexicana coronata. Roftum Vulturis. $\mathrm{O}$. culi membrana nictitante. Sub ingluuie pennae albae, quas iratus dinnittit vsque ad pedum digitos. Alae Caudaque Jibtus albo nigroque punctatac, colore Tigridis. Erectus confidet. Pennas occipitis faepius erigit in forman coronae. Fertur vnico istu cranium bominis ivatus findere. Vifus Madriti in Viuario Regio a $Z$. Hallinan.

Papa. 3. V. naribus carunculatis, vertice colloque denudato.

Vultur elegans. $E d$ w. all. 2. t.2.

Vultur. Alb. au. 2. p.4.t. 4 .

Habitat in India occidentali.

Obf. Vultur. Alb. au. 3.p.I.t.1. an femina buius?

Caput et Collum quafi excoriata retrabere poteft intre waginam cutis plumofae colli inferioris.

Aura. $\quad 4 . \mathrm{V}$. fufco-grifeus, remigibus nigris, roftro albo. Tzopilotle r. Aura. Hernand. mex. 33I. 
I. Acerpitres L. 173.5:

40. Pultur. Klein 1950. 

Vrubu brafilienfibus. Marcgr. braf. 207. Willıgh. orn. 68. Rai. au. 10. 180.

Buteo fpecie gallo-pauonis. Catesb. car. I. p. 6. t. 6.

Vultur gallinae africanae facie. Sloan. iam.2.p. 294.t. 254 .

Vultur pullus, capite implumi, cute craffa rugofa vltra aperturas nafales laxata. Brown.iam. 47I.

Habitat in America calidiore.

Privilegio munitus in Iamaica.

barbatus. 5. V. albidus, dorfo fufco, iugulo barbato, roftro incarnaro, capite linea nigra cincto.

Vultur aureus. Gefin. au. 738.t. 781.

Vultur barbatus. Edw. au. I06. t. 106.

Habitat in Africa.

Ad bafin maxillae inferioris barba dependet; frons oculorumque regio atra.

Serenopterus

Pereno- 6. V. remigibus nigris margine exteriore (praeter extimas) pterus. Percnopterus f. Grypaëtos. Aldr. orn.t.2.c. Io. Rai. aul. 8.

Falco montanus aegyptiacus. Haßelqu. act. Stockh. 1751. p. 196.

Vultur Percnopterus capite nudo, gula plumofa. Haffelq. itin. 209.

Vultur niger. Rai. au.9.

Vultur baeticus. Rai. an. IO.

Vultur albus. Rai. au. IO.

Vultur aquilina. Aldr. orn. Alb. au. 2.p.3.t.3.

Vultur fuluus, baetico bellonii congener. RAi.a a . Io. Habitat in Aegypto.

Mas totus albus: Remigibus atris margine exteriore canis, exceptis duabus prinis vnicoloribus: Femins tota $f u f c a$ : Remigibus quatuor extimis concoloribus. Roftrum atrum cera fiaua. Hafjelqu. Naves perpetuo fillant. Rai.

Purgat Caivi terram a cadaucrum foctori, fipendiis allectus. Hafelq. 
4I. FALCO. Roftrum aduncum, bafi cera inftructum. Caput pennis arcte tectum. Lingua bifida.

\section{* Cora lutea.}

Melanae- I. F. cera lutea, pedibus femilanatis, corpore ferrugineotus. nigricante ftriis flauis.

Melanaetus f. Aquila valeria. Rai. an. 7. n. 4.Will. orn. 30.t.2. Alb. all. 2. p. 2. t. 2.

Habitat in Europa.

Chryfä- 2. F. cera lutea, pedibus lanátis, corpore fufco ferrugitos. neo vario, cauda nigra bafi cinereo-vndulata. Faun. fiec. 56 .

Aquila chryfaëtos. Will. om. 27. t. I. Rai. a». 6. $n$. I. Aldr.orn.t. 2. c. 2.

Habitat in Europa.

fuluus. 3. F. cera flaua, pedibus lanatis, dorfo fufco, cauda fafcia alba. Aquila fulua $f$. Chryfaëtos, cauda annulo albo cincta. Will. orn. 28. t. r. Rai. au. 6. n.2. Hubitat in Europa.

canaden- 4. F. cera flaua pedilusque lanatis, corpore fufco, cauda fis. alba apice fufco. Aquila cauda alba. Edwo. an. I. t. I. Habitat in Canada. Pectus punctis trigonis adfper.fun.

ruftico- 5. F. cera palpebris pedibusque luteis, corpore cinereo allus. boque vndulato, collari albo. Habitat in Suecia.

barbarus. 6. F. cera pedibusque luteis, corpore caerulefcente fufco* que maculato, pectore immaculato, cauda fafciata. Falco tunetanus. $A l b . a l l .3 \cdot p \cdot 2 . t .2$. Habitat in Barbaria.

caerule- 7. F. cera palpebris pedibus fubtusque luteus, dorfo nigrofcens. caerulefcente, temporibus linea alba inclufis. Accipiter minimus benghalenfis. Edw. au. 108. t. Ic8. Habitat in Afia.

8. F.

Aquilae distae fuere Falcones maiores, pedibus birfutis. 

41. Fales L1y35 (Accipiter Keein 1950)(Aquila 

Albicilla. 8. F. cera flaua, rectricibus albis: intermediis apice nigris. Fin. Juec. 58 .

Pygargus, Albicilla, Hinnularia. Bell. au. 15. Gefn. au. 205. Aldr. orn. l. 2. c. 5. Will. ornith. 31. Rai. ail. 7.11 .5 .

B. Aquila capite albo. Catesb. car. I. t. I.

Habitat in Europa, America.

Pygar- 9. F. cera flaua, corpore cinereo, abdomine pallido magus. culis oblongis rufis, oculorum orbita atba.

Pygargus. All. all. 2. p.5.t.5. femina; et 3. p.3. t. 3. mas. Rai. au. 17.n. 5.

Habitat in Europa.

Miluus. 10. F. cera flaua, cauda forficata, corpore ferrugineo, ca. pite albidiore. Fn. fuec. 59 .

Miluus. Giefn. an. 610. Aldr. ornitb. 368. t. I. Will. ornith. 41. t.6. Rai. au.17. Albin. au. I. p. 4 . t. 4 .

Habitat in Europa, Afia, Africa.

Migrat trans pontun Euxinum in Afiam; priuilegio munita Londini. Bell. itin. IOS. Vorat quisquilias, pullos gallinaceos; tempeftates praejagit.

forfica- II. F. cera flaua, cauda forficata longiffima, corpore fupra tus. fufco fubtus albido.

Accipiter cauda furcata. Catesb. car. I. p.4. t.4. Habitat in America.

gentilis. 12. F. cera pedibusque flauis, corpore cinereo maculis fufcis, cauda fafciis quatuor nigricantibus. Fn. fusc. 60.

Falco gentilis. Will. ornith. 46 . Rai. an. 13.

Falco montanus. Will. orwith.45.t.5. Rai. as. 13.

Habitat in Alpibus, victitans Tetranoibus.

Ars copiendi Falcones Columbis et Limio, infituendi, bis venandi Gazellas, Ardeas, Auiculas etc. propriis avtificibus.

Subbu- 13. F. cera pedibusque flauis, dorfo fufco, nucha alba, teo. abdomine pallido maculis oblongis fufcis. Fin. fucc. 64 .

Sub-

Falconaria ars ad aucupium et venatum infruendi Falcones eranfit in Scientinm; inter auctores plurimos eminct d'Efparon Falconatia. Frfr. $161 \%$ quart. 
Subbuteo. Alb. al. I. p. 6.t.6. Rai. au. 15. Habitat in Europa, Alaudis infeftus.

Buteo. If. F. cera pedibusque luteis, corpore fufco, abdomine pallido maculis fufcis.

Buteo vulgaris. Gejin. au. 46. Will. ornith. 38.t.6. Rai. ait. 16.

Buteo. Alb. au. r. p. I. t. r.

Habitat in Europa, Cuniculis et Bufonibus infeftus.

Tinnun- 15. F. cera pedibusque flauis, dorfo rufo punctis nigris, culus. pectore maculis longitudinalibus fufcis, cauda totundata. Fin. Jitec. 67 .

Tinnunculus 1 . Cenchris. Gefin. au. 54. Aldr. ormith. 1.5.p. 395. Will. orn. 50.t.5. Rai. au. 16. Alb. au. I. p. 7 t.7. (femina) et 3.p.5.t. 5 .

Habitat in Europae turribus, aniculis muribusque infeftus. In cauda fafcia vnica lata sigra verfus apices.

fufflator. I6. F. cera pedibusque luteis, corpore fufco albido, oculorum operculis offeis.

Habitat Surinami. Rolander.

Corpus fupra fujcumn plumis bafi albis; fubtus luten albo fufcoque maculatum, vti etiam rectrices. Nares lobo carneo intercalari. Iratus aut territus caput inflat ad magnitudinem corporis.

cachin- I7. F. cera pedibus luteis, palpebris albis, corpore fufco nans. albidoque vario, annulo nigro verticem album cingente. Rolander.

Habitat in America meridionali.

Corpus Jupra Dorfin, Alas, vropygium fufcum ; fed Alba junt collum, gula, pectus, abdomen, alac fubtus. cauda fafciis luteis nigrisque. Hominem obferuans cacbinnos edit.

fparue- 18. F. cera lutea, capite fufco, vertice abdomineque rurius. bro, alis caerulefcentibus.

Accipiter minor. Catesb. carol. I. p.5.t.5.

Habitat in America.

columba- I9. F. cera lutea, corpore fufco fubtus albido, cauda fur rius. $\quad f \mathrm{ca}$ fafciis linearibus quatuor albis.

Accipiter palumbarius. Catesb. car.I.p.3.t.3.

Habitat in America. 


Lanarius. 20. F. cera lutea, pedibus roftroque caeruleis, corpore fubtus maculis nigris longitudinalibus. Fn. fuec. 6 I.

Lanarius. Giefn. an. 76. Aldr. ornith. 1. 5. c. II. Will. orn. 48. Kai. all. 15. Alb. all.2. p.7. t.7.

Habitat in Europa; migratorius.

* * Cera obfcura.

Haliaetus. 2I. F. cera pedibusque caeruleis, corpore fupra fufco fub. tus albo, capite albido. Fn. Jiec. 57.

Falco cyanopoda, Gefni. au. 74 .

Haliaetus. Aldr. ornitb. l. 2. c.3.

Morphnos. Aldr. ornith. l. 2.c.8.

Balbufardus. Will. ornitb. 37.t.6. Rai. au. 16.

Habitat in Europa, buni inter arundines.

Victitat pifcibus maioribus, Anatibus. Pes finifter Jubpalmatus.

Gyrfal- 22. F. cera caerulea, pedibus luteis, corpore fufco: fubco. tus fafciis cinereis, caudae lateribus albis. Faun. fuec. 62 .

Gyrfalco. Will. ornith. 44? Rai. ait. I3.?

Habitat in Europa; Columbis infeftus.

apiuorus. 23. F. cera nigta, pedibus feminudis flauis, capite cinereo, caudae fafcia cinerea apice albo. Fn. fuec. 66.

Buteo apiuorus f. vefpiuorus. Will. ornith. 38. t. 3 . Rai. all. I6.

Habitat in Europa; viçitans Muribus, Lacertis, Insjectis.

aerugino-24. F. cera fufca, corpore grifeo, vertice gula axillis pefus. dibusque luteis.

Falco cera luteo-viridi, pedibus luteis, corpore ferru. gineo, vertice fuluo. Fin. Jisce. 63.

Miluus aeruginofus. Will. orm. 42.t.7. Rai. an., 17. Alb. att. 1. p. 3. t. 3 .

Habitut in Europa.

palumba-25. F. cera nigra margine pedibusque flauis, corpore fis rius.

Aco, reetricibus tafciis pallidis, fuperciliis albis.

Accipiter palumbarius. Aldr. ornitb. 6.5.c. 2. Rai. ass. 18. n. I. Alb. all. 2. p. 8.t.8.

Habitat in Europa, Gallinis infeftus.

Subtus totus albus nigro andulatus. Regrices apice albace. 
Nifus. 26. F. cera viridi, pedibus flauis, pectore albo: fufco vndulato, cauda fafciis nigricantibus. Fn. fuec. 68.

Accipiter fringillarius. Gefin. au. 5r. Aldr. ornith. 1.5. c. 3. Bell. au. i9.6. Will.ornith.51.t.5. Rai.au. 18. Alb.ail.3.p.4.t.4. et I. p.5.t. 5. femina.

Mofcheutus. Aldr. vrnith. 345 .

Habitat in Europa; Columbis Pafjeribusqui infeftus; venatur egregie Alaudas, eoque vtinnur in aucupiis.

42. STRIX. Roftrim adincum (absque cera) bafi pennis fetaceis tectum.

Caput grande auribus oculisque magnis. Lingua bifida.

* Auriculatae.

Bubo. I. S. capite auriculato, corpore ruffo. Fn. fuec. 45 .

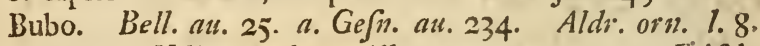
c. 2. Will. orn. 62. Alb. au. 2.p.9.t.9. Frifcb. aut. $t .93$.

Habitest in Europa.

fcandia- 2. S. capite auriculato, corpore allido. Fn. fuec. 46.

ca. Habitat in Alpibus Lapponiae.

Afio. 3. S. capite aurito, corpore fupra ferrugineo fubtus cinereo, alis punctis quinque albis.

Notua aurita minor. Catesb. car. l. p.7.t.7.

Habitat in America.

Otus. 4. S. capite auriculato pennis fenis. Faun. fuec. 47.

Otus f. Afio. Bell. au. 25. 6. Aldr. orn. I. 8. c. 3. Will. ornith. 64. t.12. Rai. au. 25. Fvifch. au. t. 99. Alb. au.2. p. 10. t.10.

Habitat in Europa.

Criftae auritae albidac fafciis fex fufcis.

Scops. 5. S. capite auriculato penna folitaria.

Scops. Aldr.ornitb. l.8. c. 4. Rai. au. 25.

Habitat iu Europa.

Strix differt a Falcone, vt Phalaena a Papilione; altera enim diurna, alter nocturnus eft.

Noßtu praedantur auiculis, muribus; die caecutinnt; pedes lanati ne frigefant; axres amplae rt percipiant; die auiculis opprobrio expofitar. 


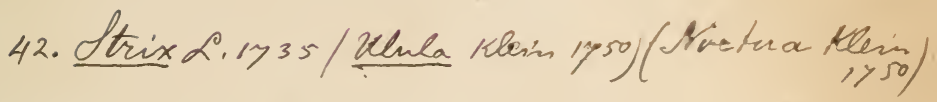


* * Inauriculatae.

Aluco. 6. S. capite laeui, corpore ferrugineo, iridibus atris, remigibus primoribus ferratis. Fn. Suec. 48. It.vel.69.* Aluco minor. Will. ornith. 67. t.13. Rai. au.25.11.1. Alb. all. 2. p.II. t. II.

Habitat in Europa.

funerea. 7. S. capite laeui, corpore fufco, iridibus flauis. Fn. fuec.5 Vlula flammeata. Frijch. au. t. 98?

Hilbitat in Europa.

Nyctea. 8. S. capite laeui, corpore albido maculis lunatis diftantibus fufcis. Fn. Jüc. 54 .

Aluco albus diurnus. Edw. aw. 61. t. 6I.

Habitat in Europa et America Jeptentrionali.

Alba fereque imnaculata in Alpibus Lapponicis.

ftridula. 9. S. capite laeui, corpore ferrugineo, remige tertia longiore. Fin. fuec. 55 .

Strix. Aldr.ornith. 561. t.563. Will. omith. 65. Rai. ali, 25 .

Habitat in Europa.

Vlula. IO. S. capite laeui, corpore fupra fufco albo maculato, reetricibus fafciis albis. Fn. Jiec. 52 .

Vlula. Gefin. au. 773. Aldr. ornith. 1.8. c.6. Will. ornith.68. t.13. Rai. au. 26.n. 4 .

Habitat in Europa.

pafferina. Ir. S. capite laeui, remigibus albis: maculis quinque ordinum. Fn. Juec. 53.

Noctua minima. Gejin. au. 620. Aldr. ornith. 1. 8. c. 7 . Will. ornith.69. t.13. Rai. au. 26. n. 6. Alb. au. 2. p. 12. t.12. Frifch. au. t. 100.

Habitat in Europa.

43. LANIVS. Roftrm rectiufculum, dente vtrinque verfus apicem, bafi nudum. Lingua lacera.

criftatus. I. L. cauda cuneiformi, capite criftato, corpore rufefcente, fubtus fuluo fufcoque vndulato.

Lanius fuluus criftatus. Edro. aw. 54. t. 54 .

Habitat in Benghala. 
Excubi- 2. L. cauda cuneiformi lateribus alba, dorfo cano, alis tor. nigris macula alba.

Ampelis caerulefcens, alis caudaque nigricantibus. $F n$. fuec. $18 \mathrm{I}$.

Lanius cinereus maior. Aldr. orn. l. 5.p.386. Will. orn. 53.t. 10. Rai. au. 18. Alb. au. 2. p. 13. t. 13 . Pica cinerea. Frifch. au. t. 59.

Habitat in Europa.

Accipitres aduentuntes objeruat et auiculis indicat.

Collurio. 3. L. cauda fubcuneiformi, dorfo grifeo, rectricibus quacuor intermediis vnicoloribus, roftro plumbeo.

Ampelis dorfo grifeo, macula ad oculos longitudinali. Fn. fiec. 180. t. 2. f. 180.

Lanius tertius. Will.ornith.54. Rai.au. 18. Alb.au. 2. p. I4. t. I4. I5.

Pica media, Lanius medius. Frifch. au.t. 60.

Habitat in Europa.

Scarabaeos Pruno Jpinofa perforat; cerebra auicularum effodit earum Simia.

Tyran- 4. L. vertice nigro: Atria longitudinali fulua.

nus. Mufcicapa corona rubra. Catesb. car. I. p.55. t.55.

Pica americana criftata. Frifch. au. 4. t.62.

Habitat in America Jeptentrionaii.

Color obfcure cinereus, abdomine albo.

Carnifex. 5. L. corpore rubro, fafcia oculari, remigum rectricumque apicibus nigris.

Garrulus ruber furinamenfis. Edw. *u. 39. t. 39.

Habitat Surinami.

Schach. 6. L. corpore lutefcente, fronte alisque nigris.

Lanius A-fcack. Osb. iter. 227.

Habitat in China. P. Osbeck.

Magnitudo praecedentiun. Caput et Collum fupra grifeum. Collum fubtus teftaceo-albidum. Dorlum et Àbdomen pallide teftacea. Remiges nigrae: primores albae; fecundariae apice albicantes.

Senator. 7. L. cauda integra, corpore fupra nigro fubtus albo, occipite purpureo.

Lanius rubro capite. $A l b$. a $a .2 . p .15 \cdot t .16$.

Habitat in Indiis. 

43a. Ampelis $\alpha .1355$. 
Faficia frontis et bafeos alarum alba. Macula alba in remigibus.

caerule- 8. L. cauda forficata, corpore nigro caerulefcente, abdofcens.

Lanius cauda forcipata. Edwo. au. 56.t.56.

Habitat in Benghala.

iocofus.

9. L. cauda integra, corpore grifeo, palpebra inferiore purpurea, ano fanguineo. Amocn. acad. 4. p...

Habitat in China. Osúeck.

Caput nigrum. Gula aiba. Abdomen albicans.

Roftrum rectius quam in reliquis, fed vtrinque intro apicen emarginatum.

Garruius. I0. L. remigibus fecundariis apice membranaceo colorato, cauda integra.

Ampslis remigibus quibusdam apice membranaceo terminatis. Fn. fuec. 179 .

Garrulus bohèmicus. Giefin. all. 703. Aldr. orn. l. I2. c. 13. Will. orn.90. t.20. Rai. aul.85. Alb.au.2. p.25.t.26. Frifch. aul. 32.f. I.

B. Garrulus carolinenfis. Catesb. car. 1. p. 46. t. 46.

Habitat in Europa et America boreali; aeftiuat fupra Sueciann.

Vibriffae ad roftrum mullate, quenadmodum in Turdis, a quibus etian Rngtro breatiore apice magis incuruo differt; Nares fetis tectae vt in Coruis; proxime autem dubia auis Laniis accedit; facies ex fafcia oculari non repugnat, fid baccitura, nec rapax. Remiges fecindariae fquama colorata terminatae. Nutritur Baccis Sorbi. 


\section{P I C A E.}

Roftrum fubcompreffum, conuexum.

44. PSITTACVS. Roftrum adtuncuìn: 'mandibủlà fuperiore mobili cera inftructa. Lingua carnofa, obtufa, integra. Pedum digiti antici 2, pofticique 2.

* Macrouri cauda cuneiformi.

Macao. I. P. macrourüs ruber, remigibus fupra caeruleis, fubtus rufis, genis nudis rugoíis.

Pfittacus cauda cuneiformi, temporibus nudis rugofis. Syft. nat. 18 .

Pfittacus maximus alter. Aldr. ornith. l. Ir. c.3. Will. ornitb. 73. Rai. au. 29. Alb. au. I. p. II. t. II. Edw. au. $158 . t .158$.

Pfittacus erythrocyaneus. Ge $\mathrm{fn}$. au. $72 \mathrm{I}$.

Araracanga. Marcgr. braf. 206.

Habitat in America meridionali.

Corpus magnitudine Gallinae, coccineunn. Roftri maxilla fuperior alba; inferior nigra. Tempora nuda, alba, rugofa. Remiges imprimis exteriores caeruleae: tectricibus fluribus luteis. Cauda cuneiformis, longiffina. Rectrices rubrae, lateralibus caeruleis.

Ararau- 2. P. macrourus fupra caeruleus, genis nudis; lineis pluna. mofis.

Pfittacus cauda cuneiformi, temporibus nudis : lineis plumofis. Syft. nat. Is.

Pfittacus maximus cyano-croceus. Aldr. ornith. l. Ir. c.2. Will. ornith. 110. t. 15. Rai, all. 28. 181. Sloan. iam. 2. p. 296. Edw. all. 159.t. I59.

Ararauna. Marcgr. braf. 206. Will. ornith. III. Alb. au. 3. p. 10. t. 10. fem. et 2. p. 16. t. I7. mas.

Habitat in America meridionali. 
Iicae 21735

44. - Prittaceno L.1535.- 

Caerulea Supra collum, Dorfun, Alas, caudam. Subtus Collo, Pectore, Abdomine, Cauda Lutea feminae, $f$. Rubra Maris. Nigra Roftrum et Gula. Palpebrae Jerratae papillis nigris. Tempora muda, lineat a funItis planofis caeruleo - nigris.

obfcu- 3. P. macrourus niger, genis nudis, vertice cinereo-nigrerus. fcente vario, cauda cinerea. Hafjelq. iter. 236 . Habitat in Africa.

nobilis. 4. P. macrourus viridis, genis nudis, humeris coccineis. Muf. Ad. Fr. 2. p.

Pfittacus viridis, alarum cofta fuperne rubente. Aldr. orn. l. II. c. 5. Rai. au. 30, 181. Sloan. iam. 2. p. 297 .

Pfittacus mediae magnitudinis. Will. orn. II2. t. I6. Habitat in America meridionali.

secerses.

feuerus. 5. P. macrourus viridis, genis nudis, remigibus reetricibusque caeruleis fubtus purpurafcentibus. Muf. Ad. Fr. I. p. I3.

Habitat in Indiis.

borneus. 6. P. macrourus ruber, remigibus rętricibusque apice viridibus, alis macula caerulea.

Plittacus coccineus, cauda longiore. Ediv. au. 173 .

t. 173 .

Habitat in India: Borneo.

folftitia- 7.P. macrourus luteus, alarum tectricibus viridibus, caulis. $\quad \mathrm{da}$ forficata.

Plittacus angolenfis. Alb. au. 3. p.13.t. 13 .

Habitat in Guinea.

caroli- 8. P. macrourus viridis, capite collo genibusque luteis.

nenlis. Plittacus carolinenfis. Catesb. car. I. p. II. t. II. Habitat in Carolina, Virginia.

alexan- 9.P. macrourus viridis, collari pętoreque rubro, gula dri. nigra. Amoen. acad. 4. p.

Pfittacus cubicularius, cauda cuneiformi, digito interiore poftice truncato. Hafjilq. itin. 235 .

Pfittacus iauanicus. Ofb. itei. IOI.

Pfittacus torquatus macrourus antiquorum. Will. orn. 77. t. 16.f: 3 . 
Pfittacus viridis ex ind. orientali. All. au. $2 . t$. Is. et 3 . t. I4.

Pfittacus torquatus macrourus antiquorum. Aldr. au. $l$. II. c. I2. Rri. au. 33 .

Perrocello. Olin. au. 24. t. 24 .

Habitat in China, Benghala, Aethiopia.

Primus (totus viridis torque miniato) innotuit fub expeditione Alexandri magni. Plin.

pertinax. IO. P. macrourus viridis, genis fuluis, remigibus rectricibusque canefcentibus. Mul. Ad. Fr. I. p. I4.

Pfittacus minor viridis, cauda longa et malis croceis. Frifch. au. $4 . t \cdot 54$.

Habitat in Indiis.

canicula- II. P. macrourus viridis, fronte rubra, occipite remigibusris. que extimis caeruleis.

Pfittacus capite rubro caeruleoque. $E d w$. au. 176. $t$. 176.

Tui-apute-iuba. Marcgr. braf. 206. n.2.

Habitat in America.

Magnitudo Turdi. Cauda corpore longior.

aerugino-12. P. macrourus viridis, vertice remigibusque primoribus fus. caeruleis.

Pfittacus minor, gutture fufco, occidentalis. Edwo au. I77. $t$. 177 .

Habitat in America.

Canda corpore breaior.

rufiro-

Aris.

13. P. mactourus viridis, roftro pedibusque rubris, rectricibus apice caerulefcentibus. $M u \int . A d . F_{3} .2 . p .$.

Pfittacus minor macrourus totus viridis. Aldr. ornith. l. 2. c. 13. Rai. au. 33. Sloan. iam. 2. p. 297.

Pfittacus minor viridis, cauda longiore. Edw. al. 175 . t. 175 .

Tuiaputepiuba. Marcgr.braf.206.n.3. Will. ornith. II6. Rai. au. 34 .

Habitat in America.

ornatus. I4. P. macrourus luteo-viridis, occipite gula pectoreque rubris, vertice auribusque caeruleis. 


Plittacus minor e coccineo viridis. Edv. all. 174. t. 174 .

Habitat in America.

agilis. 15. P. fubmacrourus viridis, tętricibus remigum primo. rum caerulefcentium fuluis, cauda fubtus rubra.

I'fittacus minor viridis. Edw. all. 168. t. 168 .

Habitat in America.

Magnitudo Coccotbrauftis. Cauda cuneiformis, fed non elongata.

* * Bracbyuri cauda aequali.

criftatus. I6. P. brachyurus albus, crifta dependente flaua.

Pfittacus albus criftatus. Aldr. ornith. l. II. c. 4. Will. ornith.. . Rai. au. 30. Seb. mus. I. p. 94. t. 59.f. I. Alb. au. 3.p. 12. t. 12. Edw. au. 160. t. 160. Frijch. au. $4 . t .50$.

Habitat in China.

niger. $\quad$ 17. P. brachyurus niger.

Pfittacus niger. Edwo. au. 5.t. 5 .

Habitat in Madagafcar.

Cauda longa, Jed aequalis.

fordidus. I8. P. brachyurus fubfufcus, gula caetulea, alis caudaque viridibus, roftro anoque rubris.

Pfittacus furcus. Edw. all. 167.t.167.

Habitat in Mexico.

erythro- I9. P. brachyurus cinereus, vropygio, ręricibus remigileucus. busque coccineis. $t$

Plittacus erythroleucus. Aldi。 orn. l. II. c. î. Will." orn. 76. Rai. an. 31.

Habitat... .

Magnitudo Gallinae.

erithacus. 20. P. brachyurus canus, temporibus albis, cauda coccinea. Muf. Ad. Fi. 1. p. 14.

Pfittacus cinereus f. fubcacruleus. Aldr. ornith. 1. Ir. $c$. 10. Rai. all. 31, Alb. al, 1. p. 12. t. 12. Frifis, alt. $4 . t .5 \mathrm{r}$.

Habitat in Guinea. 
garrulus. 2I. P. brachyurus ruber, genibus alisque viridibus, reiricibus medietate poftica caeruleis. Muf. Ad. Fr. 2. p. Pfittacus ruber, remigibus rectricibusque introrfum purpureis. It. Wgot. I37.

Pfittacus rufus, femoribus et alis viridibus. Frifch. au. 4. t. 45 .

Pfittacus coccineus, alis e viridi et nigro variis. $E d w$. aul. I72. t. 172 .

Habitat in Afia.

aurorae. 22. P. brachyurus coccineus, alis viridibus nigrisque, rectricibus flauis medietate poftica virefcentibus.

Pfittacus coccineus orientalis, alis ex viridi et nigro va. riis. Rai. au. $3 \mathrm{I}$.

Habitat in India orientali.

Corpus coccineum, magnitudine Turdi. Alarum tectrices virides. Remiges nigrae margine exteriore viridi; fubtus purpurajcentes. Annulus viridis fupra gemua. Pedes nigri. Roftrum flaumm. Rai.

Domi- 23. P. brachyurus ruber, pileo fufco, alis viridibus, hucella. meris genibusque caeruleis.

Pfittacus coccineus orientalis, vertice nigro. Edw. au. I7I. $t$. I7I.

Pfittacus brafilienfis. Alb. au.I.p.13.t.13?

Habitat in Afia.

Lory. 24. P. brachyurus purpureus, pileo nigro, alis viridibus, pectore genibus caudaque caeruleis.

Pfittacus coccineus orientalis, vertice nigro. $E d w$. all. I70.'t. 170. Frifcb. alı. 4. t. 44?

Habitat in India orientali.

caerulocephahs.

caeruleo- 25. P. brachyurus, capite pectore dorfoque caeruleis, vencephalus.

Pfittacus verficolor f. erythrocyaneus. Aldr. 0\%\%. l. in.

c. 9. Will. orn.75. Rai. au. 31.

Habitat...

leucoce- 26. P. brachyurus viridis, remigibus caeruleis;' 'fronte phalus. alba.

Pfittacus leucocephalus. Aldr. ornith. l. II. c.8. Rai. au. 3 I.

Pfittacus viridis, fronte alba, collo rubro. Frifch. au. 4. t. 46 . 


Pfittacus viridis, capite albo. Edwo. alı. 166, t. 166.

Habitat in America.

Corpus magnitudine Cuculi, viride, pennis margine nigris. Caput Jupra et ad latera caeruleum; fronte oculorumque regione alba. Gula tota rubra. Remiges nigrae latere exteriore denudato caevuleae. ReEtrices aequales, virides, bafi rubrae, apice flanefcentes; at I. I. latere exteriore caeruleo; 6. 6. vnico. acstives lores. Abdomen poftice firruginetum.

aeftiuus. 27. P. brachyurus viridis, fronte caerulea, humeris fanguineis.

Pfittacus viridis melanorhynchos. Aldr. air. l.II. c. 7 . Rai. au. 30.

Pappagalio. Olin. aur.23.t. 22.

Pfittacus viridis et luteus barbadenfis. Alb. all. 3 . p. II. t. Ir. malc.

Plittacus viridis maior occidentalis. Edwv. ant. 162. t. 162.

Pfittacus viridis, capite luteo, fronte caerulea. Fr.ifch. ali. $4 . t .47^{\circ}$

Habitat in America.

Corpus magnitudine Columbae, viride, dorfo adfperjo pennis luteis. Facies flaua. Frons caevulea. Vertex albidus. Reetrices virides apice pallidiores: I. 2. 3. bafi interiore rubrat; at I etian latere exteriore caerulea. Huneri fului f. Janguinei. Remiges primores nigrac apice caerulcicentes: latcre extcriore verfis lafin rubrae. Roftum nigrum.

paradifi. 28. P. brachyurus luteus, angulo abdominis rectricibusque bali rubris.

Pfittacus paradifi ex cuba. Catcsb. car. I. p. 10. t. I0. Restivus Habitat in America.

feltiuus. 29. P. brachyurus viridis, fronte purpurafcente, fuperciliis gulaque caeruleis, dorfo fanguineo.

Habitat in Indiis.

Corpus magnitudine Columbae.

Pfittaci monogami, per paria incedunt, garruli, loquaces, dociles, longaeni, viditant imprimis nucibus, glandibus, fem. cucurbitarum, Carthami; fcandunt roftro, irati pennas erigunt, meditabundi. 
brafilien- 30 . P. brachyurus viridis, facie rubra, temporibus caeruleis.

fis. Pfittacus viridis brafilientis. $E d w$. all. 16I. t. 16I.

Habitat in Braflia.

Auis viridis flano contaminata. Rubrum infra flexuram alae et in exteriore latere rectricum 2.2 ; caeruleum in latere exteriore renigun rectricumque primortm.

autum- 3I. P. brachyurus viridis, fronte remigumque macula cocnalis. cinea, vertice remigibusque primoribus caeruleis.

Pfittacus viridis minor occidentalis. Edvo. au. I64. t. 164

Habitat in America.

Magnitudo Columbac. Corpus viride. Oculorum regio caerulea. Alarum tectrices primores caevuleae, bafi mibrac. Remiges primores caeruleae, pofteriores virides. Reetrices fupra virides, apice flaucfentes: extimo latere exteriore caeruleo; fubtus fiauae, bafi rufoficentes, medio macula viridi.

accipitri- 32. P. brachyurus viridis, capite grifeo, collo peetoreque nus. fubuiolaceo vario, remigibus rectricibusque apice caeruleis.

Plittacus orientalis capite accipitris. Edw. au.165. $t$. 165.

Plittacus clegans. Cluf. exot. 365 . Rai. au. 32. Will. ornith....

Habitat in India.

melano- 33. P. brachyurus viridis fubtus luteus, pileo nigro, pectocephalus. Te albo. Mul. $A d$. Fr. 2. p.

Pfittacus coccineus, ventre albo. Edw. au. 169. t. I6g.

Habitat in Mexico.

collarius. 34 . P. brachyurus viridis, collo rubente.

Pfittacus minor, collo miniaceo. Sloan. iam. 2. p.297. Rai. au. ISI.

Habitat in America.

pullarius. 35 . P. brachyurus viridis, fronte rubra, cauda fulua fafcia nigra. Muf. Ad. Fr. 2. $p$.

Pfittacus paruus viridis, ex india orientali. Alb. au. 3 . p.15.t. 15 .

Pfittacus minimus viridis, cum fronte et gula rubra. Frifch. all. 4. t. 54. 
33. Jibelanocephalos 01764 . M. A. F. 10.15 
45. Ramphustos L. y40. (Nasutus Klein 1750) (Piperivorus Klein 1750.) 
Pfittacus pufillus viridis aethiopicus. Rai. au. $3 \mathrm{I}$.

Pfittacus minimus. Clus: exot. 36 \%.

Pfittacus minor verficolor. Seb. mur. 2. p. 40. t. 40. f. $\mathrm{I}$.

Habitat in Afia, Aetbiopia.

Galgu- 36. P. brachyurus viridis, vropygio pectoreque coccineis, lus. vertice caeruleo. Amoen. acad.4.p.. Osb. iter. ror. Pfirtacus minimus viridis ruber indicus. Edw. ait. 6. t. 6.

Auicula cechini. Aldr. orn. l.20.p. 560 .

Hubitat in India.

Pede altero fufpenfus dornit. Plin. $X, 33$ : Cialgulos ipfos dependentes pedibus fomnum capere confirnat, quia tutiores ita fe fperent.

pafferi- 37. P. brachyurus luteo virens, macula alarum alisque nus. - fubtus caeruleis. Muf. Ad. Fr. I. p.I4. Habitat in America. Magnitudo Pafferis.

Rewniges fubtus et tecirices alarum primariae caeruleae.

45. RAMPHASTOS: Rofrum maximum, inane, con- vexuni, extlorfum ferratum. Nares pone maxillas. Lingua pennacea. Pedum digiti antici pofticique gemini.

piperiuo- I. R. roftro nigro: carina craffifima. rus. Habitat in America meridionali.

Tuca- 2. R. roftro rubro: carina obtufa alba. nus. H.abitat in America meridionali. Maxillae verfus bafin fafcia nigra.

picatus. 3. R. roftro rubro: apice nigro: carina compreffa.

Pica brafilienfis. Alb. au. 2. p. 24. t.25. Aldr. om. l. 20. c. 19.

Hubitat in America meridionali.

Roftrum magaum inter congeneres; ctian bafi nigricans. 
Aracari. 4. R. roftro nigro: maxilla fuperiore lateribus alba, bafi, triloba.

Toucan. Ed. au. 64. t. 64 .

Aracari. Marcgr. braf. 217. Rai. au. 44.

Habitat in America meridionali.

Maxilla fuperior ad nares triloba, quod non in reliquis fafcia abdominis anoque fanguincis.

46. BVCEROS. Roftruni connexum, cultratum, magnum; Frontis caluaria nuda, ofleo-gibbofa.

Nares in bafi roftri.

Lingua acuta, bieuis.

bicornis. I. B. fronte offea plana antrorfum bicorni. Annoen. acad. 4. $p$...

Calao. Pet. gaz. 43. t. 28. f. 6. et t. 31. f. I. Will. ornith. t. I7. f. I.

Habitat in China.

Corpus magnitudine Gallinae, nigrum; fubtus pectore, abdomine, femoribus albuni. Macula alba in remrigibus alarim. Cauda longiufcula. Reetrices 10, nigrae, extimis vtrinque albis. Pedes virefcentes: digitis 3 anticis; I poftico.

Rhino- 2. B. cornu maxillari frontis recuruato. $f$

ceros. Rhinoceros. Bont. iau. 63. t. 64. Olear. mus. t. I5.f. 4. Will. orm. t. 17. f. 3. Worm. mut. 293. Aldr. orm. l. 12. c. 20.

Habitat in India.

Cum Willugbbaeus aliique roftrun extrorfum ferratum fiftant, videtur Rampbafti potius generis anis effe. Foeda foctidaque cadaueribus victitans venatores co. mitatur. 
46. Buceros L. 1940.- 
47. Crotophuga L.1350.

40! Cowno L.1735/Pica Klein 1750). 
47. CROTOPHAGA. Rofirum compreffum, femiouatum, arcuatum, fulco vtrinque exaratum; Mandibula fuperiore margine vtrinque angulata. Nares peruiae.

Ani.

I. C RO TOPHAGA.

Crotophagus ater, roftro breuiore compreffo arcuato cul. trato. Brown. iam. 474.

Monedula tota nigra maior garrula, mandibula fuperiore arcuata. Sloan. iam. 2. p. 298.t.256. f. I. Catesb. cas: 3. p. 3. t. 3.

Ani. Marcgr.braf. 193. Will. orn. 120. Rai. au. 35. Habitat in America, Africa; victitans gryllis.

48. CORVVS. Toftrum conuexum, cultratum, bafi pennis fetaceis tectum. Lingua cartilaginea bifica.

Corax. I. C. ater, dorfo caerulefcente, cauda fubrotunda. $F_{n}$. fuec. 69 .

Coruus. Gefin. all. 334. Aldr. orn.1. 12. c. 1. Ionft. aus. 38. t. 16. Will. ornith. 82. t. 18. Rai. au. 39. All. all. 2. p. 19. t. 20. Firigch. all. t. 63.

Habitat in Europa, viçitat cadaueribus, furto deditus, loqui addifcit, feris conuintalis, clamitans.

Corone. 2. C. atro-caerulefcens, cauda rotundata: reetricibus acutis.

Cornix. Rai. au. 39. n.2 Alb. au.2.p. 20. t.2I.

Habitat iu Europa.

frugile- 3. C. ater, fronte cinerafcente. Fn. frec. 70.

gus. Cornix frugilega. Aldr. orn. l. 12. c. 3. Will. ornith. 84. t. 18. Rai. au.39. All.an. 2. p. 21. t. 22. Frijcb. ail. t. 64 .

Habitat in Europa, agris infefta, gregaria.

Cornix. 4. C. cinerafcens, capite gula alis caudaque nigris. $F_{n}$. fiec. $7 \mathrm{I}$. 
Cornix cinerea fruģilega. Aldr. ornitb. l. I2. c. 4. Will. ornitb. 84. t. 77. Rai. au. 39. Alb. au. 2. p. 22. t. 23. Frifch. au. t. 65.

Habitat in Europa, victitat Laruis Tipularum, quisquiliis; apud nos relegata, at inaudita et indefenfa. Ventis aduerja confidet.

Mone- 5. C. fufca, occipite incano, fronte alis caudaque nigris. dula. Fin. Succ. 72 .

Monedula f. Lupus. Aldr. orn. l. 12.c. 9. Will. ornith. 85. t. 19. Rai.au. 40. Alb. au. I. p. 14. t. I4. Frifch. cu. t. 67.

Habitat in Europae agris; gregaria.

bengha- 6. C. vertice alis caudaque caeruleis, collo grifeo.

lenfis.

Pica glandaria benghalenfis. Alb. au. I. p. I7.t. I7.

Habitat in Benghala.

glanda- 7. C. tectricibus alarum caeruleis: lineis transuerfis albis rius. nigrisque, corpore ferrugineo variegato. Fr. Juec. $74^{\circ}$. Pica glandaria. Gejn. au. 700. Aldr. omith. l. 12. c. 14. Will. om. 88. t. 19. Rai. au. 4.1. Alb. au. I. p. I6. t. 16. Frifch. au. t. 55. Olin. au. 35 .

Habitat in Europa; colligit nuces, glandes; fuperfiuns defodit.

criftatus. 8. C. teetricibus alarum lineis transuerfis nigtis, corpore caeruleo, collari nigro.

Pica glandaria caerulea criftata. Catesb. car. p. 15. t. I5. Habitat in America Jeptentrionali. Praecedenti in multis affinilans.

Curyoca- 9. C. fufcus alboque punitatus, alis caudaque nigris: retactes. Ctricibus apice albis: intermediis apice detritis.

Coruus cinereus, cauda alisque nigris. Fn. Juec. 75 .

Cáryocatactes. Gefn. au. 245. Will. ornith. 90. t. 20. Rai. au. 42 .

Pica abietum nigra guttata. Frifcb. all. $t .56$.

Habitat in Europa; edit nuces.

Pica, Io. C. albo nigroque varius, cauda c:ıneiformi. Fr. fuec. 76 .

Pica varia f. caudata. Gefn. au. 695. Aldr. orn. l. I2. c. 12. Ionft. au. 44. t. 17. Will. ornith. 87.t. I9.Rai. Bu. 41. Alb. all. t. I. p. 15. t. I5. Frifch. au. t. 58. 

49. Coracias 2.1435 
Habitat in Europa nido artificiofo.

Degit ad pagos, inter boftes offenfos, fyluarum ofor; 18. git quisquilias.

paradifi. II. C. albo nigroque varius, cauda cuneiformi, remigibus intermediis longiffimis, capite nigro criftato.

Manucodiota criftata ex albo nigroque variata. Rai. all. I95. t.2.f. 13 .

Auis paradifi. Seb. muf. I. p.85.t. 52.f.3. et $t .30 . f \cdot 5$. Pica orientalis, cauda duabus pennis longifimis. Edw. au. 113 . t. 113 .

Habitat in India.

infauftus. 12. C. dorfo cinereo, reetricibus rufis: intermediis duabus cinereis, fafcia nigricante.

Turdus rectricibus rufis: duabus intermediis cinereis fafcia nigricante; proximus apice cinereis. Fn. füec. 187. Merula faxatilis. Will. orn. 145.t. 36. Rai. all.65. Alb. au. 3. p. 5I. t. 55 .

Habitat in Europae alpinis fyluis.

Auis proterua, faepę carniuora.

49. CORACIAS. Roftrum cultratum apice.incuruato, bafi pennis denudatum. Lingua cartilaginea, bifida.

Garrulus. I. C. cacrulea, dorfo rubro, remigibus nigris.

Coruus dorfo fanguineo, remigibus nigris, ręricibus viridibus. Fin. Juec. 73 .

Cornix cuerulea. Giefri. aur. 335. Aldr. ornith. l. I2. c. 18. et 5. Will. orn. 85. t. 20 .

Garrulus argentoratenfis. Rai. all. 41. Edw. au. 109. t. 109.

Habitat in Europa, vitatans Scarabaeis, Ranis.

caffra. 2. C. caerulea, remigibus margine exteriore luteis.

Habitat in Aerhiopia. F. Burmannus.

Femina caerule fcente- nigra cft.

Oriolus. 3. C. flaua, alis caudaque nigris.

Ampelis flaua, artubus nigris, ręricibus quinque extcrioribus retrorfum flauis. Ad?. Stockh. 1750. p. 127. t. $3 . f \cdot 5$.

Oriolus. Gefin. all. 132. 
Galbula. Aldr. orm.854.t.857.858.

Galbula benghalenfis. Eáw. au. 185. t. 185 .

Picus nidum fufpendens. Will. orn...t.36. f.6. et t. 38 .

f. 4. Ionft. alt. II2. t. 4I. f. 8. Rai. au. 68.

Flaua auis, benghalenfis. Alb. au. 3.p.19.t. 20.

Habitat in Europa, Afia migratoria auis.

Galbula. 4. C. fulua, capite dorfo remigibusque nigris.

ICterus ex aureo nigroque varius. Catesb. car. I. p. 48. $t, 48$.

Habitat in America.

aurea. 5. C. flauo-fulua, gula tectricibus primariis extremitateque rectricum nigris.

Paradifea flamo-fulua. Muf. Ad. Fr. I.p. I5.

Paradifea aurea. Edw. au. II2. t. II2.

Habitat in Afia.

Xanthor- 6. C. flaua, capite remigibusque primoribus nigris.

nus. IEterus indicus, capite nigro. Edm. au. 77.t. 77 .

Xanthornus maior, nigro varius. Brown. iam. 477.

Pica luteo nigra varia. Catesb. car. $3 \cdot p \cdot 5 \cdot t \cdot 5$.

Habitat in America.

50. GRACVLA. Roftrum conuexo" cultratuin, bafi nudiufculum.

Lingua integra, acutiufcula, carnofa. Pedes digitis anticis 3 , poftico $\mathbf{I}$.

religio- I. G. nigro violacea, macula alarum alba, fafcia occipitis fa. nuda flaua.

Coruus iauanenfis. Osb. iter. ro2.

Sturnus indicus. Bont. iau.67. Will.orm. $445 \cdot$ t. 38.Rai au. 68.

Minor f. Mino. Edw. au. 17. t. 17. Alb. au. 2. p. 35. t. 38 .

Habitat in Afia.

foetida. 2. G. nigra, remigibus extus caerulefcentibus, fafcia collari nuda.

Habitat in: America. Rolander. 
-

50. Gracula L. is58 

Magnitudo Picae. Corpus nigrum, at Alae rompofitis remigibus extus cacrulefcentes absque ulba macula. Rectrices aequales. . Caput nigrum, plunnis erectis breuiffimis quafi bolofericeun. Rofrum fere Cuculi. Nares vuatae mudue. Lingua integra crirnofa acuta. Pedes tctradactyli: vno poffico.

Barita. 3. G. fubgrifea, humeris caeruleis, remigibus extus viridibus.

Habitat in Americae Mufis, cuius fructus deuaftat. Ro. lander.

Rofrum breuturfculunn, cultratum, nigricans, bafi nudum, fubtus albicans. Alae complicatis remigibus virides; explicatae remiges intus nigrae. Cauda rotundata, viridis dun complicata.

criftatel- $4 \cdot$ G. nigra, remigibus primoribus bafi rectricibusque apice la. albis, roftro flauo.

Sturnus chinenfis niger. Edw. au. xg. t.19.

Habitat in China.

Saularis. 5. G. nigro-caerulefcens, abdomine macula alarum rętricibusque lateralibus albis.

Pica minor benghalenfis. Edw. aul. I81. t. 181.

Dialbird. Alb. au. 3. p. 17. t. 17. I8.

Saularis maderafpatanus. Rai. au. 197.t. 2. f. 19. 20. Habitat in Alia.

Quifcu- 6. G. nigro-violacea, cauda fubcuneiformi.

la.

Monedula purpurea. Catesb. car. I. p.12. t.12.

Merops niger, iride argentea. Brown。ian. 476 .

Habitat in America fiptentrionali.

Virginianis inuifa auis; aqua et igne interdicta, fed iniuffe.

Atthis. 7. G. viridi-caerulea, abdomine fetrugineo, pedibus fanguineis.

Coruus aegyptius viridi maculatus, dorfo medio caeruleo. Haffelq. iter. 140. n.20.

Habitst in Aegypto. 
5x. PARADISAEA. Roftrum baif plumis tomentofis tectum.

Pemae hypochondriorum lon. giores.

apoda. I. P. pennis hypochondriis corpore longioribus, reetricibus intermedis longis fetaceis.

Paradifea lutea, cauda pectore abdomineque brunneis, gula azurea. It. Wgoth. 139. Muf:Ad. Fr. 1. p. 15.

Paradifea auis. Cluy. exot.360.t. 360 . Olear. muf: 24 . t. 15. f. I. Seb. mut. I. p. 24. t. 13. f. I. Hern. mex. 3i7.t.318. Worm. muf: 294.f. 294. Eradl. mat.t. 12. f.1. 2.

Manucodiata maior. Edwo. au. IIO. t. IIO. Marcgr. braf. 20I. Rai. aut.2I. 11.7.

Ilabitat in India; gregaria.

Cirrbi 2 caudac apice attentati, nudi, recti.

Apodam perperans dixere veteres; victitat Papilionibus maximis.

regia. 2. P. cirrhis caudalibus filiformibus apice lunato-pennaceis. Muf. Ad. Fr. I. p.15.

Rex auium paradifaearum. Pet. gaz. I. t. 53.f. 2. Chuf: exot. 362. t. 362. Olear. mul. 24. t. I3. f. 2. Rai.au. 22. 12. IO. Seb. muf. I. t. 38.f. 5. Edw. all. III. t. III.

Habitat in India oricntali.

Purpurea pectorali caerulefcente; cirribi caudae longiffimi ; Pemae fub alis junt reliquis longiores, licet cauda breniores. $\left({ }^{*}\right)$

52. CVCVLVS. Roftrum tèretinfartim:

Nares margine prominulae.

Lingua fagitrata, plana, integra.

sanorus. x. C. cauda aequali nigricante albo punctata. Fn. Juec. 77. Cuculus. Gejin. au. 363. Aldr. ornitb. 1. 5. c. 107. Bell. au. 22:6. Will. ornith. 62. t. 77. Frifcb. au. 4.t. 40, 4I, 42. Rai. all. 23. Alb. all. I. p.8.t.8. Olin. ans. 38 .

Habi-

(") Paradifaeas fictas (Seb. muf. I. t. 60. f. $I, 2,3$ ), e Pfittacis aliisquue asudidit Jaepe Mufsis afthe chinersium. 
51. Paradioaea 20.1335 (Paradioéa fron .1756.)

52. Cuculun 21435 
52.a. Touraco Klein nso (Taurace Klain) 
Habitat in Europa; vicfitat Laruis.

Oua fua aliarum auicularum nidis imponit, cubandi impotens; variat actate, fexu; tempore frondefcentiae, flurefcentiae, grolf? ficationis cuculat; occultatur caniculae ortu; in falconem transformari perperans afjeritur.

Perfa: "2. C. cauda aequali, capite crifta eręta, remigibus primoribus rubris.

Touraco. Alb. au. 2.p. Ig.t. I9. Edw. au. 7. t.7.

Habitat in Africa.

Antice viridis, poftice fubuiolaceus, remigibus primoribus rubris; linea alba jupra et infira palpebras janguineas.

Vetula. 3. C. cauda cuneiformi, corpore fubfufco: fubtus teftaceo, ciliis rubris.

Cuculus maior. Sloan. iam. 2. p. 3I2. t. 253. $f . \mathbf{x}, 2$.

Cuculus maior oliuaceus, cauda longiore, ciliis rubris. Bromn. iam. 476.

Picus maior leucophaeus $\mathrm{f}$. canefcens, pluuiae auis et $S e$ nex dictus. Rai. au. 182.

Habitat in Iamaica.

Voce clamofiore pluniam praejagit.

glanda- 4. C. cauda cuneiformi, capite fubcriftato, fafcia oculari rius. nigra.

Cuciulus fuluus maculatus. Edw. au. 57.t. 57 .

Habitat in Africa Jeptentrionali et Europa auftrali.

- Dorfum fufcum. Alae albo punctatae. Subtus teftaceus eft. Caput cinercum, fibcriftatum. Linea nigra trans oculos.

Scolopa- 5. C. cauda cuneiformi, corpore vndique grifeo fufcoque ceus. nebulofo.

Cuculus indicus fufcus maculatus. Edw. all. 59. t. 59 . Habitat in Benghala.

niger. 6. C. cauda cuneiformi, corpore nigro nitido, roltro flaun.

Cuculus indicus niger. Edro. au. 58.t.58.

Habitat in Benghala.

america- 7. C. cauda cuneiformi, corpore fupra cinereo: fubtus alnus. bo, maxilla inferiore lutea. 
Cuculus carolinianus. Catesb. car. 9.t.9.

Habitat in Carolina.

auratus. \&.C. cauda fubforcipata, gula pectoreque nigris, nucha rubra.

Picus maior, alis aureis. Catesb. car. I. p. I8. t. I8. Habitat in Carolina.

Cauda refert Picum. Auis varia pulcbra; rachis remigum primorum aurea.

53. IYNX.

Roftrum teretiufculum, acuminatum. Nares concauae.

lingur teres, lumbriciformis, longiflma, apice mucronata.

Torquil- I. I Y $\mathrm{N}$.

1a. Cuculus.fubgrifeus maculatus, rectricibus nigris fafciis vndulatis. Fn. Jivec. 78 . $t$. I. $f .78$.

Iynx. f. Torquilla. Bell, au. 76. a. Gefn. al. 573. Aldr. ornitb. l. I2. c. 42. Ionft. alk. . t. 42. Will. orn. 95. t. 22. Rai. au. 44. Rudb. lapp. 66. t. 66. Alb. au. I. p. II. t. 2I. Frifcb. au. ..t. 38 .

Habitat in Europa.

54. PICVS. Roftrum polyedrum ${ }_{0}$ rectum: apice cuneato.

Nares pennis fetaceis obtectae.

Lingua teres, lumbriciformis, longiffima, mucronata, apice retrorfum aculeata fetis.

martius. I. P. niger, pileo coccineo. Faun. fuec. 79.

Picus niger maximus. Gefn. au. 708. Aldr. orn. l.12.

c. 3r. Will. ornitb. 22. t. 2r. Rai. all. 42. Alb.

iut. 2. p. 26.t.27. Frifch. alt. t. 34.f. I.

Habitat in Europa, vix in Anglia.

Arbores emortuas confcindit.

Lingua Picorum et Iyngis inferitur frontì. Inteftina caecis carent. 
s. Jognx 2. 19\$8..

54. Rieno 2.1435. 

principa- 2. P. niger, crifta coccinea, linea vtrinque collari remigilis. busyue fecundariis albis.

Picus maximus, roftro albo. Catesb.cav. I. p. 16. t. 16. Habitat in America feptentvionali.

pileatus. 3. P. niger, eapite criftato rubro, temporibus alisque albis maculis.

Picus niger maximus, capite rubro. Catesb. car. 2. p.

17. t. 17. Kalm. itin.2. p. 27 I.

Ipecu. Marcgr. braf: 207.

Habitat in America.

Differt a P.cornicino, quod tempora alba ; maculae aliquot paruae in alis albae: caput magis late coccincurit.

hirundi- 4. P. niger, occipite coccineo, humeris albido puntatis: naceus. Picus fecundus niger. Alb. all. 3.p. 22. t.23.

Hubitat in America feptentrionali.

Magnitudine vix Hirundinem fiperat.

erythro- 5. P. capite toto rubro, alis caudaque nigris, atdomine cephalus. albo.

Picus capite toto rubro. Catcsb.car. I. p.20. t.20.

Habitut in America.

caroli- 6. P. pileo nuchaque rubris, dorfo fafciis nigris, rectricinus. bus mediis albis nigro punctatis.

Picus ventre rubro. Catesb. car. I. p. 19. t. 19. f. z.

Habitat in America feptentrionali.

Ani regio rubra punctata.

viridis. 7. P. viridis, vertice coccineo. Fn. fuec. 80.

Picus viridis. Gefin. all. 710. Alitr. ornitls. l. 12.c.34. Will. ornitb. 93. t. 21. Rai. all. 42. Alb. all. 1. p. 18. t. IS. Frifch. all...t. 35. f. I.

Habitat in Europa.

hengla- S.P. viridis, occipite rubro, nucha nigra, fubtus anticeque lentis. albus nigro maculatus.

Picus benghalenfis. Alb. au. 3.p. 21. t. 22. Edw. au. 182. 1. 182.

Habitat in Benghala.

Pici Laruas infedtorum lignum intus rodentinm roftro secante, fono terrefaciente, auditu percipiente, lingua acsia baftata intrants cxitalownt, de qua $A$ It. angl. 350. p. sog. 
Habitus $P$. viridis fed dilutior; facie antice, collo fubtus, pectore albis maculis nigris. Cauda nigra. Occiput crifta rubra.

femiro- 9. P. maxilia fuperiore breuiore. Muৎ. Ad. Fr. 1. p. 16.

ftris. Habitat in India.

major.

maior. Io. P. albo nigroque varius, ano occipiteque rubro.

Picus albo nigroque varius, reftricibus tribus lateralibus vtrinque albefcentibus. Fn. Juec. 8r.

Picus varius maior. Gejn. au. 708 . Aldr. urnith.l. I2.

c. 32. Will. orn. 94. t.21. Rai. au. 43. Alb. au.

I. p. 19.t. I9. Frifch. al...t.36.f. I.

Habitat in Europa.

medius. II. P. albo nigroque varius, ano verticeque rubris.

Picus albo nigroque varius, rectricibus tribus lateralibus apice albo variegatis. Fn. fuec. 82.

Picus varius minor. Gefn. au. 708. Aldr. orn. 1. I2. c. 33. Will. ornith. 92. t. 21. Rai. au. 43. Haj:felqu. iter. 242.

Habitat in Europa.

minor. 12. P. albo nigroque varius vertice rubro, ano albido.

Picus albo nigroque varius, rectricibus tribus lateralibus feminigris. Fr. Jisec. 83. Haffelqu. iter. 242.

Picus varius tertius. Rai. au. 43 .

Picus varius minor. All. alt. I. p. 20.t.20.

Habitat in Europa.

tridakty- I3. P. albo nigroque varius, pedibusque tridactylis. Frn. lus. fuec. 84. Act. Stockb. 1740.p. 222.

Picus tridactylus. "Edvo. au. II4. $t$. II4.

Picus tridactylos anomalus. Muf. petrop. 368.

Habitat in Suecia ad Alpes Lapponicas, Dalekarlicas frequens et ad frnm Hudfonis nec non in Sibiria.

Europacus vertice flaus, Americanus rubro eft. Ani regio albida. 

55. Jitta L.1935

56. Sopida L.1735 (Alledo L1750) 
55. SITTA. Roftrum fubcultrato-conicum, rectum, porrectum, integerrimum: mandibula fuperiore obrufufcula.

Lingua lacero-emarginata.

europaca. I. S. rectricibus nigris: lateralibus quatuor infra apicem albis. Fn. Juec. 182.

Sitta 1. Picus cinereus. Giefir. au. 7II. Aldr. om. l. I2.

c. 38. Will. orititb. 98. t. 33. Rai. all. 4.7 . Alb. all.

2. p. 27.t.2S. Frifćn. all...t. 39.f. 2 .

ß. Sitta capite nigro; et Sitta capite fufco. Catesb. carol.

T. p. 22.t. 22.

Habitat in Europae, Americac truncis arborum, laruas fub cortice effodiens; Nuces roftro perforat et edit. Nidlum ftruens reperto congruo foramine arburis, illud limo oblinit pro capacitate ingre/Jus.

s6. ALCEDO:' Rofturn titgòntm, - eraflum, rectum, longum.

Lingua carnofa, breuiffima, plana, acuta.

Ifpida. I. A brachyura, fupra caerulea, fubtus fulua.

Ifpida fupra caerulea, fubtus ferruginea. Muf. Ad. $F$. I. p. 16 .

Ifpida. Rai. au. 48. n. I. Olin. all. 39. Aldr. orn. \%. 20. c. $6 \mathrm{I}$.

Ifpida indica minor. Edw. au. Ir. t. Ir. All. all. I. "s" 5I. $t .54$.

Alcedo americana f. Apiaftra. Seb. muf. I. $57 . \pm 53$. $f: 3$.

Habitat ad maris littora in Europa, Afia.

erithaca. 2. $\Lambda$. brachyura, dorfo caeruleo, abdomine luteo, capite vropygioque purpureo, guia nuchaque albis.

Alcedo benghalenfis. Alb. atl. j. p. 2S. t.29.

Habitat in Benghala.

Alcyon. 3.A. brachyura nigra, abdomine albo, peใtore ferrugineo.

Alcyon. Catesb. car. I. p. 69. t. 69. Rai. as. 49. n. 2 . et 184. "1. 14 . 
Ifpida carolinienfis. Edw. au. II5. t. II5.

Iaguacati guacu. Marcgr. braf.r94.

Hubitat in America.

Roftrum capite longius, validum $\int$. Picae. Macula alba ante et poft oculos. Lingua breeriffina, carnofa, acuta, plana. Corpus nigrum. Pennae capitis longiores. Remiges nigrae, apice albne: exterioribus minoribus; omnes latere interiore albo maculatae. Rectrices 12, aequales, vtrinque albo maculatac. Gula alba. Pectus et Abdomen alba, fed Pectus antice fafcia ferruginea. Femora ferruginea. Tibiae breuiffinae. Digitus extimus connexus cum intermedio.

Todus. 4. A. brachyura viridis, gula fanguinea, abdomine albo. Rubecula viridis elegantifima. Ediv. au. I2r. $t$. I2I. $f$. r. Sloan. iam. 2. p. 306. t. 263. f. r. Rai. au. 187 . Todus viridis, pectore rubro, roftro recto. Brown. iam. 476.

Habitat in America.

Genus dubium et quafi medium inter Alcedinem et Trochilum.

Inymen- 5. A. macroura ferruginea, alis cauda dorfoque viridibus. fis.

Alcedo fmyrnenfis. Alb. all. 3.p.26.t.27.

Ifpida maior africana. Edw. au. 8. t. 8.

Habitat in Africa et Afria.

Confer. Alcedo maior benghalenfis. Alb. au. 3. $p$. 27 I. 28.

rudis. 6. A. macroura fufca albido varia.

Alcedo rudis. Halfelq. iter. 243. $n .22$.

Ifpida ex albo et nigro varius. Edro. au. 9. t. 9.

Habitat in Perfia, Aegypto.

Cauda aequalis albida fajcia lineari lataque nigra.

Dea. 7. A. reßtricibus duabus longiffimis, corpore nigro-caerulefcente, alis virelcentibus.

Alcedo binis plumis in cauda longiffimis. Edw. au. Ió. t. 10.

Auis paradifiaca ternatana. Seb. mme. I. p. 74. t. 46 . f. 3 ?

Habitat Surinami. 

5\%. Merops Klein 1yso.

50. Uprpa 2. 1735.- 
57. MEROPS. Roftrum curuatum, compreffum, cạrinatum.

Lingua apice laciniata.

Digiti infimo articulo coadunati: poftico diftineto.

Apiafter. I. M. dorfo ferrugineo, abdomine caudaque viridi-caerulefcente, reatricibus duabus longioribus.

Merops. Bell. au. 16. Gefn. au. 599. Aldr. ornith. l. I2. c. 45. Will. ornitb. 102. t. 24. Rai. au. 49. Alb. all. 2. p. 40. t. 44. Charl. onom. 87. t. 87.

Merops galilaeus. Haffelq. iter. 247.

Ifpida. Fn. Suec. 86.

Habitat in Europa auftrali, Oriente.

Gregaria victitat Cicadis, Apibus.

viridis. 2. M. dorfo ferrugineo, abdomine alisque viridibus, gula caudaque caeruleis.

If pida viridis, fupra ferruginea. Osb. iter. 96.

Merops viridis, fupra ferruginea. Cbin. lagerftr. 5 .

Merops benghalenfis. All. au. 3. p.29. t. 30 .

Merops minor, caudae 2. pennis longioribus et tenuio. ribus. Edwo. all. 183. t. 183.

Habitat in Iaua, Benghala.

cinereus. 3. M. rubro flauoque variegatus, fubtus flauo-rubefcens, rectricibus duabus longifilimis rubris.

Auicula de guanheilui. Seb. muf. 1. p.50.t. 30. f. 10. Habitat in America.

cafer. 4. M. grifeus, ani regione flaua, cauda longiffima.

Habitat in Aethiopia.

Inter pulchre delineatas icones animaliun vltra 150 , quas mijut annicifinus I. Burmannus, vifá eff.

58. VPVPA. Roftrum arcuatum, conuexum, fubcompreflùn.

Lingua obtura, integerrima, triquetra, breuillima.

Epops. I. V. criftata variegata. 
Vpupa. Fn. fuec. 85. Bell. au. 72: a. Gefn. au. 776. Aldv. onnitis. l. 17. c. II. Ionft. au. ..t. 42. Will. ornith. 100. t. 24. Rai. all. 48. Alb. alt. 2. p. 39.t. 42.43. Frifch. au. i. 43. Charl. onom. 63. t. 63. Olin. au. 36 .

Habitat in Europae fyluis.

Terrefacta criftam erigit.

paradi- 2. V. rectricibus duabus longiffimis.

fea.

Auis paradifiaca Manucodiaca criftata orientalis. Seb. mute. . . p. 40. t. 30. f. 5 .

Habitut in Afia.

Eremita. 3. V. viridis, capite flauo, ceruice iubata.

Coruus fyluaticus. Gefin. au. 35I. Aldr. orn. l. 19. e. 57. Eremita montanus helueticus. Alb. an. 3. p. 16. t. I6. Habitat in Heluetia.

Pyrrho- 4. V. atra, rofto pedibusque rubris.

corax. Monedula Pyrrhocorax. Hafjelq. iter. 238.

Coracias f. Pyrrhocorax. Aldr. orn. l.12.c. \&. Will.orn. 86. l. 19. Rai. an. 40. n. 6. Alb. au.2. p. 23. t. 24. Habitat in Angliae, Aegypti maritinis.

59. CERTHIA, Roftrum arcuatum; tenue, fubtrigẹnum.......

\section{Linguia acuta.}

familia- I. C. fupra grifea, fubtus alba, remigibus fufcis: decem ris. macula aiba.

Certhia. Fn. fuec. 213. Gefn. au. 25I. Aldr. ornith. l. 12. c. 44. Bell. aw. 98. a. Will. orn. 100. t. 23. Rai. au. 47. n. 5. Alb. au.3.p. 24.t.25. Frijch. au. 39. $f .1$.

Habitat in Europae arboribus reptans; 0ua 20. parit. Reniges 4-I4. macula transuerfá alba notatae.

pufilla. 2. C. fupra grifea, fubtus alba, rectricibus fufcis: extimis apice albis.

Certhia fufca minor. Edw. all.26. l. 26.

Habitat in India.

eaerulea. 3. C. caerulea, remigibus reetricibusque nigris.

Certhia caerulea. Edw. แน. 2I. t. 2I. 
59. Certhia L 1735 (Falcator Klem 195) H Falcinelum Certhia chaleybeata i. NG.16.d. F. F.: = 
bo.- $\frac{\text { Troglodytes } 2.1740 .(\text { Trochiluo L.1748) (thellionga }}{\text { Hein 1750.) }}$ 
Habitat Surinami.

Roftrum buic longius quan in congeneribus.

cruenta- 4. C. nigro-caerulefcens, fubtus alba, vertice, ceruice, dort2. fo vropygioque rubris.

Certhia minor, ex albo nigro et rubro varia. Edwo.au.8I. t. 8I.

Habitat in Benghala.

flaúeola. 5. C. nigra, vropygio pectoreque luteo, fuperciliis macula alarum reetricumque apicibus albis.

Lufcinia f. Philomela e fufco et luteo varia. Sloan. iam. 2. p.307.t.259. Rai.au. I87.11.45. Edw. au.122. t. 122.

Hubitat in America.

60. TROCHILVS. Roftrum fubulato-filiforme, incuruatum, capite. longius: Mandibula fuperior vaginans inferiorem.

Lingua filiformis, bipartito-tubulofa.

* Macrouri 1-5.

paradi- I. T. curuiroftris ruber, alis caeruleis, capite criftato, refeus. Etricibus duabus longiffmis.

Melliuora auis criftata cum duabus pennis longis in cauda. Seb.muf. 1. p.97.t.6r.f. 4. .

Habitat in Mexico.

afer. 2. T. curuiroftris, corpore viridi, alis caeruleis, reatricibus nigris: intermediis longiffimis.

Habitat in Aethiopia. I. Burmannus.

Pella. 3. T. curuiroftris, rętricibus intermediis longifimis, corpore rubro, capite fufco, gula aurata vropygioque viridi.

Melliuora maior rubra, cauda longa. Edw. au. 32.t.32. f. 2 .

Habitat in Indiis.

4. $T$.

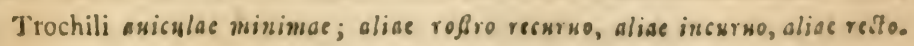


Polyt- 4. T. curuiroftris, rectricibus lateralibus longiffimis, corpomus. re virefcente, piléo ręricibusque fufcis.

Polytmus viridans auren varie fplendens, pinnis binis vropygii longiffinis. Brovon. iam. 475 .

Melliuora capite nigro, cauda longa. Edw.au.34. t.34. Alb. au. 3. p. 45. t. 49. f. I?

Melliuora auis maxima. Sloan. iann. 2.p.309.t.264. f.4. Habitat in America.

forfica- 5. T. curuiroftris, rectricibus lateralibus longifimis, cortus. pore viridi, pileo ręricibusque caeruleis.

Polytmus maior nigricans aureo varie fplendens, pennis binis vropygii longifimis. Brown, iam. $p .475$.

Melliuora viridis, cauda longa. Edwo. au. 33. t. 33 .

Melliuora auis minima. Sloan. iann. 2. p. 307. t. 264 . f. r. Rai. au. 187.

Habitat in Iamaica.

Pennae longiffinae bafi Juffultae fupra et infsa pennula rigida.

* Bracbyuri 6-18.

Colubris. 6. T. rêtricibus nigris: lateralibus tribus apice albis, gula rubra.

Melliuora auis carolinenfis. Catesb. car. I. p. 65. t. 65 .

Melliuora gula rubra. Edwo. au. 38. t. 38 .

Habitat in America, imprimis feptentvionali.

Ouriffia. 7.T. rectricibus fubaequalibus fufco-aureis, corpore aurato, remigibus nigris.

Habitat in Indiis.

Molqui- 8. T. reetricibus aequalibus ferrugineis: extimis apice fueus. fcis, corpore nigro-aureo, remigibus nigris. Mus: Ad. Fr. 2. p. 24 .

Habitat in Indiis.

holoferi- 9. T. curuiroftris rectricibus aequalibus fupra nigris, corceus. pore fupra viridi, pectore caeruleo, abdomine nigro. Melliuora viridis, ventre nigro. Edw. au. $36 . t .36$. Habitat in America.

I0. $T$.

Mel e foribus bauriunt more Bombylii, wes plantis inhaerent. 


mellifu- Io. T. rectricibus aequalibus caeruleis, corpore viridi aureo, gus. remigibus atro-caerulefcentibus. Milf: Ad. Fr. 2. p.. Habitat in Indiis.

Tomi- II. T. reetricibus fubaequalibus bafi ferrugineis apice albis, neo. Ouriflia f. Tomineo. Cluy!: exot. 96. Habitat in America.

Hanc feminam $T$. colubris (6.) putat Edro. au. $t .38$.

furina- 12. T. rectricibus ferrugineis, remigibus nigris, corpore menfis. aureo fubtus albicante.

Habitat in America.

niger. 13. T. reetricibus fubaequalibus, corpore nigro fubtus aurato, ano albo.

Trochilus niger. Mus. Ad. Fr. I. p. 18 .

Habitat in Indiis.

melliuo- I4. T. rectriroftris, rętricibus nigris: lateralibus albis, ca=

rus.

pite caeruleo, dorfo viridi, abdomine albo.

Melliuora ventre albo. Edw. au. $35 \cdot$ t. 35.f. I.

Habitat in India.

Rectrices laterales albae; Collum a tergo lunula alba.

ruber. I5. T. refricibus lateralibus violaceis, corpore teftaceo fufco fubmaculato.

Melliuora parua purpurea. Edw. au. 32.t.32. $f$. I.

Habitat Surinami.

Refirices intermediac teftaceat.

Mango. 16. T. reAtricibus fubaequalibus ferrugineis, corpore teftaceo, abdomine atro.

Melliuora Mnngn. Alb. ant. 3.p. 45.t.49.f. 2.

Habitat in Iamaica.

criftatus. 17. T. rectiroftris viridis, alis fufcis, abdomine cinereo, crifta caerulefcente.

Meiliuora minor criftata. Ediv. au. 37. t. 37.

Habitat in America.

minimus. 18. T. rectricibus, lateralibus margine exteriore albis, corpore fulco nitente, fubrus albido.

Auis melliuora minima. Edw. aw. 105. t. 105. f. infima. Habitat in America.

Auis minima inter ombnes notos. 


\section{ANSERES.}

Roftrum obtulfufculum, epidermide tectum, fub bafi giboum, apice auctum.

Lingua carnofa, obtujirifctlita.

-Pedes palmati, natatorii.

6x. ANAS. Rofrum lamellofo-dentatum, conuexum, obtufum.

Lingua ciliata, obtufa.

* Roftro bafí gibbo.

Cygnus. r. A. roftro femicylindrico atto, cera flaua, corpore albo. Fn. fuec. 88. It. wogotb. 123 .

Cygnus ferus. Bell. ant. 30. a. Gefn, ant.372. Aldr. ormith. l. 19. c. 1. Will. orn. 212...Rai. au. 136. Alb. all. 3.p. 9r. t.69. Edw. ar. 150. t. 150.

ß. Cygnus maníuetus. Will. orn. 271. t.69. Rai. au. 136. Habitat in Europa, America Septentrionali.

Accedit ad nos vltimo die regelationis. Pifcatur pomo. It. fcan. I86. 228.

Cygnoid. 2. A. roftro femicylindriso bafi gibbo. Fn. fuec. 89.

auftralis. Anfer cygneus guineenfis. Rai. au. I38. Will. orn. 275 .

Anfer cygnoides. Alb. aur. I. p. 89.t. 9r.

orienta- $\beta$. Anfer roltro femicylindrico atro bafi gibbo, minor.

lis. Anfer chinenfis. It. Wgot. I45.

Anfer mofcouiticus. Alb. au. 2.p.83.t. 9r. 92.

Habitat in Afia.

Varietas orientalis $\beta . a b$ aultrali $\alpha$. partmn differt; ins. primis quod illa minor.

Tador- 3. A. roftro fimo, fronte compreffa, corpore albo variena. gato.

Anas albo-variegata, pectoris lateribus ferrugineis, abdomine longitudinaliter cinereo-maculato. Fn. Jivec. 93 . Anas Tadorna dieta. It. Scan. 212.* It. Wgoth. 214. Tadorna f. Vulpanfer. Will. orn. 278. t. 70.71. Rai. au. $\mathrm{T} 4 \mathrm{O}$.

Vulpanfer. Alb. am. I. p. 90.t. 94 . 
Anoeres Lins

boa.Cygmm L.1935

61. Anas. 2.1735. - (Anoer Klein 19s0.) (Serrator Klicis.) 

Brandgaas. Cluf. exrot. 368 .

Habitat in Europae maritimis.

fpętabi- 4. A. roftro bafi gibbo compreffo: carina pennacea nigra,

lis. $\quad$ capite canefcente. Muf. Ad. Fr. 2. p.25.

Anas canadenfis, capite grifeo caeruleo. Edw. au. 154.

t. I54.

Habitat in Canada, Suecia.

fufca. 5. A. nigricans, macula pone oculos lineaque alarum albis. Anas corpore obfcuro, macula alba pone oculos, lineaque alba. Fn. Juec. 106. It. gotl. 215.271.

Anas fera fufca. Ionfl. au. t. 44 .

Anas niger. Will. orn.278.t. 10. Rai. all. I41.

Habitat in oceano Europaeo.

Mas ad bafin roftri gibbofitate notatus.

nigra. 6. A. tota nigra, bafi rofri gibba.

Anas niger minor. Will.orn. 280.t.74. Rai.au.14t. Habitat in Lapponia, Anglia.

Cauda fibcuneiformis. Femina fufca eft.

* * Laeues roftro bafi aequali.

Anfer. 7. A. roftro femicylindrico, corpore fupra cinereo fubtus pallidiore, collo friato. Fin. fuec. 90.

๙. Anfer ferus. Gefin. all. 158. Allr. orn. 1. 19. c. 18. Will. orn. 274. t.69. Rai. att. 136.

B. Anfer domefticus. Gefn. at. 141. Will. orn. 273. t. 75 . Rai. aut.136.

\%. Anfer canadenfis fufcus maculatus. Edwo. au. 153.t. 153 . Habitat in Europa et America'maxime boreali.

Collum friatum. Annulus albus ad bafin rofiri in Spontaneo; migrat per phalanges; filo arcetur. Mas I feminis 4 ; inde pernae, plumae, Anfer durateus, Ius Jpurtanum, lecur ficatum.

erythro- 8. A. cinerea, fronte alba. Fn. fuec. 97

pus. Anfer hellingicus. Cluf. exot. 368.

Habitat in Europa Jeptentrionali.

canaden- 9. A. fufca, capite colloque nigro, gula alba.

fis. Anfer canadenfis. H'ill. ornith. 276. t. 70 . f. I. et t. $7 x$. f. 3. Rai. aut.139.t. 10. Catesb. sar, 1. p. 92. t. 92. Edro. all. 15 I. t. 15I.

Habitar in Canada. 
caerule- Iо. A. grifea, fubtus alba, tectricibus alarum dorfoque pofcens.

ftico caerulefcentibus.

Anfer canadenfis, alis caeruleis. $E d 20$. au. I52.t. 152. Hubitat in Canada.

Bernicla. Ir. A. fufca, capite collo pectoreque nigrris, collari albo. Anas capite colloque nigris. Fn. fuec. 91.

Branta f. Bernicla. Gefn. au. IIO. Aldr. orn. l. I9.

c. 23. Ionft. aut.t. 49. Will. orn. 274. 275. t. IO.

Rai. au. 137. Valent. mul. 495. Alb. au. I. p. 39. t. 93.

Habitat in Europa boreali; migrat fupra Suecian.

mollifi- 12. A. roftro cylindrico, cera poftice bifida rugofa. Fn. ma. fuec. 94.

Anas plumis mollifimis. Barth. aft. I. p. 90. Wornm. mis. 310. Will. orn. 277. Rai. au. I4r.

Anas magna nigra et alba. Edwo. au. 98.t.98.

Habitat in Eutopa boreali, pelagica. It. gotl. 213. deplumatur in vido: plumis molliffmis, praeftantiffmis, pretio/ifjenis.

Nius albus, fubtus pofticeque niger. Femina grifea.

mofcha- I3. A. facie nuda papillofa. Fn. fuec. 98.

ta.

Anas molchata. Rai. au. 150. Alb. au. 3. p. 92. t. 97.98 .

Anas indica. Gefin. au. I22. Aldr. orm.l. Ig. c. 26.

Anas libyca. Will. orm.294.

Habitat in India.

Alba buius forte varietas minor. It. Wgotb. I43.

bahamen-14. A. grifea, roftro plumbeo: macula laterali fulua, ala-

fis. $\quad$ rum viridi luteaque.

Anas bahamenfis; roftro plumbeo, macula aurantii coloris. Catesb. car. I. p.63.t.93.

Habitat in Bahama.

Albeola. 15. A. alba, dorfo remigibusque nigris, capite caerulefcente, occipite albo.

Anas minor albus et fufcus. $E d w$. all. 100. $t .100$. Habitat in America.

clypeata, I6. A. roftri extremo dilatato rotundaso, vngue incuruo. Fr. Jupc. 102. It. gotl.167. 


Anas latiroftra maior. Gefn, au. I20. Aldr. orn. 8. p. 227.

Anas platyrhynchos altera. Will. 0rm.283, t. 74. Rai. ail. 143 .

Anas clypeata germanica. Alb. al. I. p. 9j, t. 97. 98. Habitat in Europae maritimis.

platy- 17. A. macula alari purpurea vtrinque nigra albaque, perhynchos. Etore rufefcente. Fn. firec.103.

Anas platyrhynchos, pedibus luteis. Aldr, orn. l. Ig.c. Will. orn. 284. Rai. all. I44.

Habitat in Europae maritimis.

ftrepera. I8. A. macula alarum rufa nigra alba. Fn. fuec. IOI.

Anus ftrepera. Gefin. au. I2I.

Anas platyrhynchos, roftro nigro et plano. Aldr. orn. 1. 19. c. 32. Rai. all. 145 .

The gottwall or gray. Hill. orn. $287, t, 72$.

Habitat in Europae aquis dulcibus.

bucepha- I9. A. albida, dorfo remigibusque nigris, capite fupra in. la. fraque tumido fericeo nitente.

Anas minor, purpureo capite. Catesb. car. I. p.95.t.95. Habitat in aquis aulcubus Americae jeptentriunalis.

Clangu- 20. A. nigro alboque varia, capite tumido nigro-viridi:

1a. finu oris macula alba. Fin. fiuec. 100. it. oel. 49.

Clangula. Gejir. au. II9. Aib. au. I. p. 92 t. 96.

Anas platyrhyrichos mas. Aldr. orn. l. 19. c.42. Will. orn. 282. t.73. Rai. au. 142.

Habitat in Europa; Jaepias maritima.

Vrinatur egregic, quacitura cucbleas, tracbea ventrico. ja, oculis aeneis.

ruftica. 2I. A. fufco cinerea, macula aurium alarumque alba.

Anas minor ex albo et tulco varia. Catesb. car. I. p. 98. t. 98 .

Habitat in America Septentrionali.

perfpicil-22. A. nigra, vertice nuchaque albis, macula nigra roftri lata. pone nares.

Anas canadenfis maior miger. Edr. aน. 155. $t$. 155 .

Habitat in Canada. 
Glau- 23. A. iridibus flauis, capite grifeo, collari albo. Faun. cion. juec. 104 .

Glaucion. Bell. an. $33: 6$. Aldr. orn. l. 13. c. 38 . Will. orn. 231. Rai. ant.144.

Habitat in Europae maritinis.

Penelo-24. A. cauda acutiufcula fubtus nigra, capite brunneo, pe. fronte alba. Fn. Juec. I05.

Penelope. Gefn. au. I08. Aldr. orn. 1. 19. c. 38. Ionft. au. l. 49. Will. orn. 288. t.72. Rai. alt. 146.

Anas fiftularis. Alb. au.2. p. 88. t. 99.

Habitat in Europae maritimis et paludibus.

acuta. 25. A. cauda acuminata elongata fubtus nigra, occipite vtrinque linea alba.

Anas cauda cuneiformi acuta. Fn. Juec. 96.

Anas cauda acuta. Gefin. au. 121. Will. orn. 279. t. 73. Rai.all. 147. Alb. all.2.p. 84.t.94.

Habitat in Europae mpritimis.

hyema- 26. A. cauda cuneata: rectricibus intermediis longis, cora lis. pore grifeo, temporibus albis. Fn. Juec. 95.

Anas islandica. Worm. mus. 302.

Anas cauda acuta canadenfis. Edwo. au. 156.t. 156.

Habitat in Europa et America arctica.

Hiene ad nos migrat; aefate verfis Septentrionem vlterius pergit.

ferina. 27. A. alis cinereis immaculatis, vropygio nigro. Faun. jiter. 107.

Anas fera fufca. Gefri. au. I16. Aldr. orn. l. Ig.c. 40. Will. 0rn. 288. t.72. Rai. au. 142. Alb. au.2. p. $87 . t .98$.

Habitat in Europae maritimis.

Querque-28. A. macula alarum viridi, linea alba fupra oculos. Fn. dula. fiec. ros.

Kernell. Gejin. alt.108. Aldr. orn. l. 19.c.33.

Querquedula prima. Will. ornitb.291.t.74. Rai. as. I48.

Habitat in Europae aquis dulcibus.

Crecca. 29. A. macula alsrum viridi, linea alba fupra infraque ocue los. Fn. fitec. Iog. 


Querquedula fecunda f. minor. Gefin. au. 105. Aldr. orn. I. I9.c.31. Will. orn. 290. t.74. Rai. aul. 147. Querquedula francia. Alb. all. I. p. 95. t. 100. et 2. p. 9r. t. I02.

Habitat in Europae aquis dulcibus.

hiftrioni- 30. A. fufca albo caeruleoque varia, auribus, temporibus ca. linea gemina, collari fafciaque pectorali albis. Anas fufcus maculatus. Edwo. all. 99. t. 99. Habitat in America.

minuta. 31. A. grifea, auribus aibis, remigibus primoribus nigricantibus.

Anas canadenfis minor fufcus. Edho. an. 157. t. 157 . Habitat in Canada.

Parua admodum, tota grifea. Punctun albmin ad aures vtrinque. An prioris femina?

Circia. 32. A. macula alarum varia, linea alba fupra oculos, roftro pedibusque cinereis. Fin. futec. III.

Anas Circia. Alb. all. 2.p. 92.t. 103. 104. Rai. al. 143 .

Habitat in Europae paludibus.

autumna-33. A. grifea, remigibus cauda ventreque nigris, atea alalis. $\quad$ rum fulua albaque.

Anas fiftularis rufus, roftro rubro, occidentalis. Edw. ail. I94. $t$. 194 .

Habitat in America.

* * Pennis aliquod recuruis.

Bofchas. 34. A. reetricibus intermediis (maris) recuruatis, roftro recto. Fin. Jituc. 97.

Anas vera torquata minor. Gefin. all. IIf. Aldr. orn. l. 19. c. 25. Rai. alt. 145 .

Bofchas mainr. Will. urn. $284 \cdot$ t.72. Alb. au. 2. p. 39. $t$. 100.

B. Anas domeltica. Ionft. all. I4r. t. 49. Will. orn. 293. t. 75. Aldr. orn. l. 19. c. 35. Gicji, all. 96. Rai. au. 150 .

Halitat in Furopae lacubus; et inanfueta.

Mas autunno feminis Jimilis; ona mumerofa parere incipit fiorefcinte Hepatica. Viatitat Ramis, Culicibus, Lemina. 
adunca. 35. A. rectricibus intermediis (maris) recuruatis, roftro incuruato.

Anas domeftica, roftro adunco. Will.orn. 208. Rai.au. 150. Alb. au. 2. p.86. t.97.96.

Habitat in .., bodie vti pracedens domeftica.

Quomodo baec a priori exorta fit, me fugit.

galericu- 36. A. crifta dependente, dorfoque poftico vtrinque penna lata. recuruata compreffa eleuata.

Querquedula finenfis elegans. Edho. au.IO2.t. IO2.

Kinmodfuis. Kaempf. iap. 129. t. Io.

Habitat in China.

Crifta zividis mubraque. Roftrum et pedes rubri. Dorfum fufcum maculis caevuleis. Pennae dor/i erectae rufae obtufia. Remex intimus, claufis alis, alteromargine evigitur jupra dorfun, rufus antrorfum falcatus.

**** Crijtatae.

Sponfa. 37. A. crifta dependente duplici viridi caeruleo alboque varia.

Anas criftatus elegans. Catesb. car. I. p. 97. t. 97. Edw. au. IOI. t. IOI.

Anas fufca criftata, ad gulam alba, iride flammea. Brown. ian. 48r.

Habitat in America feptentrionali.

Crifta dependens viridi alboque caerulea, vti et dorfum. Pectus griféum, albo maculatum. Gula alba.

arborea. 38. A. grifea, capite fubcriftato, abdomine albo nigroque maculato.

Anas fiftularius rufus, roftro nigro, occidentalis. $E d w$. ak. I93. $t$. 193 .

Anas fiftularis arboribus infidens. Sloan. iam.2.p.324. t. 272. Rai. au. 192.

Habitat in America.

Fuligula. 39. A. crifta dependente, corpore nigro, ventre maculaque alarum albis. Fn. Juec. 99.

Anas Fuligula. Gefn. an. 107. Will. orn. 280.t.73. Alb. au. I. p. 9r. t. 95 .

Anas criftata. Rai. au. I42.

Habitat in Europae maritimis.

Mas, incubante femina, nos deferit. 

62. Werguo 2.1335 (Derrator Klein'yso.) 
62. MERGVS. Rºfrumi denticulatum, fubulato-cylindricum apice adunco.

eucuila- I. M. crifta globofa vtrinque alba, corpore fupra fufco, tus. fubtus albo.

Anas criftatus. Catesb. car. I. p. 94.t. 94 . Habitat in America.

Mergan- 2. M. crifta dependente, capite nigro-caerulefcente, collafer. ri albo. It. gotl. 166. oel. 49. Fn. fuec. II3. Merganfer. Gefin. au. 134. Aldvo orn. l. 19. c. 63. Will. orn. 253.t.64. Rai. au. I34. Marj: danub. 78. $t .37$.

B. Mergus maior, pętore rubro. Alb. all. I. p. $83 . t .87$. et 2. p. yo. t. IOI. Edw. all. 95. t. 95 .

Habitat in Europa.

Serrator. 3. M. crifta dependente, capite nigro maculis ferrugineis. Fin. filec. II4. It. gotl. $27 \mathrm{I}$.

Anas longiroftra. Gefin. au. 133. Aldr. orn. l. 19. ‘. 60. Mergus cirrhatus fufcus. Will. orn. 255.t. 64. Rai. aut. 135 .

Habitat in Europa.

Hulius methodus pifiandi babetw in AEtis Stockb. 1749 . p. 190; rubro allicitur.

Albellus. 4. M. crifta dependente fubtus nigra, corpore albo, dorfo nigro, alis variegatis.

Mergus Tinus. Hafjelq. iter. 268.

Mergus inaior cirrlatus. Rai. au. I35.

Serrator minimus, capite niueo, ceruice genisque nigris. Klein. au. I40.

Albellus alter. Aldr. orm. l. 19. p. 378. Alb. all. I. p. 85.t.89. Will. orn. 254. t. 64 .

Habitat in Europa.

minutus. 5. M. capite grifeo laeui. Fn. fuec. II5.

Te wezel-coot. Alb. au. l. p. 84. t. 88.

Habitat in Europa.

In alis macula atra, antice et pofice alba. 
63. ALCA. Róftrim edentalum, breûe, compreffum, conuexum, fulcatum faepius transuerfe: Mandibula inferior ante bafin

gibba.
Pedes plerisque tridactyli.

Torda. I. A. roftri fulcis quatuor, linea vtrinque alba a roftro ad oculos. It. gotl. 286. Fn. Sulec. 120.

- Alca. Chuf: exot. 367. Worm. muf. 363. Will. orn. 243. t. 64.f. 1. et t. 65. f. 2. Rai. alt. I19. Alb. atl. 3. p. $90 . t .95$.

Habitat in Europae borealis oceano.

Plures oua folitaria fimul in antro rupium pariunt, coniunct is viribus alternatis excludunt, vnica excubias agente; erectae incedunt.

impen- 2. A. roftro compreffo-ancipiti fulcato, macula ouata nis.

vtrinque ante oculos. Fn. finec. IIg. Anfer magellanicus. Worm. muf: $300 . t .301$.

Penguin. Will. ornith. 244.t. 65. Edwo. Mu. 147.t. 147. Habitat in Europa aretica.

aretica. 3. A. roftro compreffo - ancipiti fulcato fulcis quatuor, oculorum orbita temporibusque albis. Fn. fuec. II8.

Anas arctica. Cluf: exot. I04. Rai. ali. I20. Worm. mul. 302. Olear. muf: t. 15. f.5. Will. orn. 244 . t. 65. Rai. all. 120. Alb. aul. 2. p. 73.t. 78. 79 .

Lunda. Gefin. au. 725 . Oelig. mul:" 2. S. II. to. II. f. $2 \mathrm{r}$.

Lomvia

Habitat in Europae borealis oceano.

Lomuia. 4. A. roftro laeui oblongo: mandibula fuperiore margine flauefcente.

Lomuia. Chuf. exot. 367. Will. om. 244.t.65. Rai. ail. 120.

Guillemot. Alb. au. I. p. 80.t.84.

Habitat in Europa boreali.

Grylle. 5. A. roftro laeui fubulato, abdomine maculaque alarum alba, pedibus rubris.

Colymbus pedibus tridactylis palmatis. Fn. Suec. I24.

Colum-

Aliae impenras, aline fingulares, quod nequeant volare.

Pennatae alise, in terva ftobuinutes, erestae incedunt. 
63. Dlea L. 1946. (Plantur Klein 1750) 
bn Proellaria $\mathcal{L} 1746$. 
Columba groenlandica. Mart. Spitzb. 56. t. L. f. B. UTill. ornith. 245. Rai. au. 12т. n. 6. Alb. au. 2. p. 73 . t. 8 o. Edw. au. 5 o. t. 50 .

Habitat in Europae borealis occano.

Roftrum buius longitudine fere capitis, fequentis vero brectiffinumm.

Alle. 6. A. roftro laeui conico, abdomine fafciaque alarum alba, pedibus nigris.

Columba groenlandica. Alb. au. I. p. 81. t. 85. Edw. ali. 9r. t. 91.

Mergus melanoleucus, roftro acuto breui. Will. 0\%\%? 261. t. 59. Rai. att. 125. n. 5.

Habitat in Europae, Americae arcticae oceano.

Praecedentis feminam efje ftatuit Albinus, negant Ede. wardus et noftrates.

64. PROCE LLARIA. Roftrum edentulum, fubcompreffumi: Maridibulis àequalibus: fuperiore apice adunca; inferiore apice compreffo- canaliculata.

Nares cylindro fupra bafin roftri decumbente, truncato. Pedes palmati: vngue poftico ferfili absque digito.

pelagica. I. P. nigra, vropygio albo. Fn. Juec. 249.t. 2. f. 249 . Act. Stockb. 1745. p. 93. t. . .

Peteril. Cluf. exut. 368 . Damp. itin.3. p. 97. All.ai. 3. p.87.t.92. Edw. an. 90. t. 90. f. I.

Hirundo marina maior apus, roftro adunco. Barr. ace quin. 134 .

Larus minimus, natibus tubulofis. Catcsb. carol. 3. p. I4. t. 14 .

Fregata marina apus fubtus alba, fuperne nigra. Barr. all. 73 .

Habitut in albo Qceano.

Proccllarine aues pelagicae fune, noc ald consinentis littora confpicisutur, expofitae onnibus procollis. 
aequino- 2. P. furca immaculata, roftro flauo.

Ctialis. Peteril magna nigra. Edw. au. 89.t.89. Habitat ad Cap. b. Spei.

capenfis. 3. P. albo fufcoque varia. Amoen. acad. 4 . p. . Osb. it. 76. Anas alia indica. Giefr.au.803.t. 804.f. 2.

Peteril media maculata. Edwo. au. 90.t. 90.f.2. Habitat ad Cap. b. Spei.

65. DIOMEDEA. Rơftrtm rectum: maxilla fuperiore apice adunca; inferiore truncata.

Naves ouatae, patulae, prominulae, laterales.

exulans. I. D. alis pennatis, pedibus tridactylis.

Albatros. Edw. au. 88. t. 88. Alb. au. 3. p. 76. t. 81. Grew. muf.t. 6. f. I.

Habitat intra tropicos Pelagi et ad Cap. b. Spei, aetbera altifine fandens; victitans e Triglis volitantibus a Corypbaena exagitatis.

Statura Pelecani Aquili, navibus ouat is prominulis, non vero linearibus; caudarotundata breui nec forficata. Roftrun in bac quadruplo maius.

demerfa. 2. D. alis impennibus, pedibus tetradaetylis.

Penguin pedibus nigris. Edw. au. 94. t.94.

Habitat ad Cap. b. Spei.

Statura Pbä̈tbontis demergi, fed roftrum diuer.fiffmum.

66. PELECAN VYS. Rofrum edentulum, witum:'apice adunco, vnguiculato.

Nares lineares, obliteratae.

Facies nudiufcula.

Pedes digitis omnibus quatuor fimul palmatis.

* Rufero edentulo.

Onocto- I. P. gula faccata. Syft. nat. 23.

talus. Onocrotalus. Olear. mus. t. 13.f. I. Dod.mem.3.p.'86. 
Gs Dismedea 2 isso.

66. Peleanus 20.1735/ Plancus Klein 1750) 
66. Graculus L. 1235 
Onocrotalus f. Pelecanus. Aldr. orn. l. 19. c. 2. will. orn. 246. t.63. Rai. au. 12r. Marfil. danub. 6. $t$. 35. Edw. au. 92. t. 92. Bont. iaur. 68.

ß. Onocrotalus $f$. Pelecanus fufcus. Sloan.iam. 2. p. 322. Rai. ati. I9I.

Onocrotalus americanus. Edwo. au. 93.t. 93.

Habitat in Africa, Afia, $\beta$ in America.

Remiges nigrae.

An americanus $\beta$ fufcus ab orientali albo Jpecie fufficienter diftinctus?

Sacco gulari aquam fiticulofis pullis adfert, it et consterranei fitim expleant intactis pullis.

Aquilus. 2. P. cauda forficata, corpore nigro, capite abdomineque albis. Amoen. acad. 4. p...

Pelecanus Aquilus. Osb. iter. 292.

Alcyon maior pulla, cauda longiore bifurca. Brown. iam. 483 .

Cari pira. Laët. amer. 575 .

Man of var. Sloan. iam. I. p. 30.

Auis Rabo focardo. Pet. gaz. t. 45. f. 1. ?

Frigate bird. Alb. all. 3. p. 75. t. 80. male.

Habitat in Infula Adfcenfionis aliisque pelagicis, vitam agens Diomedeae exullis, cui fimilis.

Carbo. 3. P. cauda aequali, ćorpore nigro, roftro edentulo.

Pelecanus corpore atro, fubtus albicante, rectricibus quatuordecim. Fn. fiuc. 116.

Carbo aquaticus. Giejin. au. I37.

Coruus aquaticus. Aldr. orn. 1. 19. c. 55. Ionft. as. t. 47. Will. orn. 248. t. 63. Rai. au. 22. Marf. danub.16.t. 36. Alb. un. 2. p.74. t. 8r.

Habitat in Europa; nidificat in altis arboribus.

Cauda longior et laxior quan in reliquis aquaticis.

* Mandibulis ferratis.

Baffanus. 4. P. cauda cuneiformi, rofto ferrato, remigibus primo ribus nigris.

Anfer Baflinus. Will. orn. 247. t. 63. Rai. al. 122. Alb. all. I. p. \$2. t. 86 .

Anferi batfano congener cinereo albus. Sloan. iam. prabf. 3I. t. 6. f. I. Rai. au. I9I.

Habitat in Scotia, America. 
Magnitudo Anferis. Ad nares, fupra oculos, pone funum oris, linea lateralis fub gula, muda.

Mandibulae fabrase, nun ferratae.

Pifcator. 5. P. cauda cuneiformi, roftro ferrato, remigibus omnibus nigris. Clinin. Lagerftr. 3. Osb. iter. 85.

Anaethetus maior melinus fubtus albidus, roftro ferrato dentato. Bromn. ian. 48I.

Anferi baffano affinis. Catesb. car. I. p. 87. t. 87 . Sloan. iam.2. p.322. t. 27I. f. 2. Rai. art. I91.

Habitat in India vtraque, Europa.

Alter fexus albus remigibus wigris, alter niger abdomine conefiente. Osbeck.

Mandibula fuperior verfus bafin margine quafi dente virinque mutilata. Nares claufae. Remiges in albo primures nigrae, fecundariae albae, fed apicibus ex. trorfun nigrae.

67. PHAËTHON. Roftritwi cultratùm, rectum, acuminatum. Fauce pone roftrum hiante.

Nares oblongae, poftice membrana femitectae.

Digiti poltici antrorfum verfi.

aethereus. I. PH. rętricibus duabus longiffimis, roftro ferrato, digito poftico adnato.

Auis tropicorum. Catesb. car. 3.p. I4. t. I4. Rai. au. I23. Will. orn. 250. t. 76. Edw. au. 149. t. 149. $f$. I. Sloan. iañ. I. p.22. Kalm. iter. 2. Ip. I49. Osb. iter.291.

Auis Rabos forcados. Aldr. om. 1. 20. p. 544 .

Habitat in Pelago inter tropicos. Osbeck.

Magnitudo Anatis, alba Jupra lineis nigricnntibus vndulata tota; Jubtus imnnaculata. Fafcia nigra per oxulos. Roftrum fanguineum, compreffenn, rectius quam P. Pifcatoris, retrorfun ferratum. Pedes pallidi, extrorfun nigri, digito quarto etian membrana affixo. Remiges omnes fubtus albae: primores fupra latere extesiore nigrae; fecundariae fupra linea longitudinali nigra. Reetrices albae in cauda cuneiformi, verfus apicem lineola fubfagittata fufca; ommes fuproi sacbi niuca.

2. PH. 
67. Phaïthon Linsos. 
Go. Colymbno 2 viss 
demer- 2. PH. alis impennibus, roftro mandibulis edentulis, digifus.

to poftico diftincto.

Penguin. Edwo. alt. 49. t. 49 .

Habitat ad Tropicum capricorni.

Remigibus defituitur, adeoque volatus nefcia auis.

6s. COLYMBVS. Roftrum edentulum, fubulatum, rectun, aciminatum.

Pedes pone aequilibrium.

ar尺ticus. I. C. pedibus palmatis indiuifis, gutture nigro-purpurafcente. Fn. fucc. I2I. It. gotl. 341.

Colymbus areticus Lumme. Worm. mus. 304. t. 305. Will. orn. 259.t. 62. Rai. au. 125. Oelig. mus. I. f: Ir. t. I. f. 2 .

Mergus maximus ferroenfis $\mathrm{f}_{0}$ ar:ticus. Cluf. exot. 102. Colymbus collo rubro. Edw. al. 97. t. 97. et 146 . t. 140.

Habitat in Europa et America boreali; lacuffris.

Mas fub gula purpurrafcens, Femina vero nigra; monogama; oun 2. in littore; nequit incedere; feris exofa; clamitando tempeftates praefagit; migrat in proximant Germaniant.

Confi: Colymbus maximus caudatus. Alb. all. I. p. 78 . t. 82. Rai. au. 125. n. 4. Will. orn. 258. t. 62. f. I. femina.

criftatus. '2. C. pedibus lobato-fifilis, capite rufo, collari nigro', remigibus fecundariis albis. Fin. furec.' 122.

Colymbus maior criftatus f. cornutus. Gefn. all. 139 . Aldr. ornitb. 1. 19. c. 52. Will. orn. 257. t. 61. Rai. aut. 124. Marjal. damb. SO. t. 3 S.

Habitat in Europa.

auritus. 3. C. pedibus lobatis, capite nigro, auribus criftato-ferrtgineis. For. Succ. I23.

Colymbus 1. Podiceps minor. Aldr. orn. l. 19. c. 52 . Will. orn. 258.t. 6r. Sloan. iam. 2. p. 322. 2. 27 I. f. 1. Rai. all. 125. Marj. damub. S2. t. 39. Alu. au. 2.p. 70. t. 76 . E.tw. au. 145. t. 145. et y6. t. 96.

Habi-

Colymbi nequenut incedere, at citibime Super agnam crrinut, et egregie ขrsmaาเนr. 
Habitat in fummis Europae ct Americae lacubus. Nidum natantem conficit.

Podi- 4. C. pedibus lobatis, corpore fufco, roltro fafcia fesquiceps. altera.

Podiceps minor, roftro vario. Catest. car. I. p. 9I. t. 9 I.

Habitut in America Septentrionali.

69. LARVS. Roftrum edentülum, rectum, cultratum, apice decliue: Mandibula inferior infra apicem gibba.

Nares lineares, antice latiores.

tridacty- I. L. albicans 'dorfo canefcente, rectricibus excepto extilus. mo nigris, pedibus tridactylis.

Larus hybernus. Alb. all.2. p. 80. t.87.

Larus cinereus. Bell. all... Rai. all. I28. n. 4 .

Habitat in Europa Septentrionali.

canus. 2. L. albus, dorfo cano. Fir. fuec. I25.

Larus albus. Alb. all.2. p.77.t. 84.

Habitat iu Europa.

Agitati Lari euomunt aut explodunt cibunn.

marinus. 3. L. albus, dorfo nigro. It. gotl. 214. It. Wgot. 156.

Larus nigrefcens marinus. Cluf. exot. 104. Alb. all. 3 . p. 89. t. 94 .

Habitat in Europa.

fufcus. 4. L. albus, dorfo fufco. Fn. Juec. 126.

Habitat in Europa.

Atricilla. 5. L. albus, capite alarumque apicibus nigris, roftro rubro.

Larus maior. Catesb. car. I. p. 89.t.89.

Habitat in America.

parafiti- 6. L. rectricibus duabus intermediis longiffimis.

cus. Sterna reetricibus maximis nigris. Fn. fisec. I29. It.

Wgot. 182. Act. Stockb. 1753. p. 291.

Auis

Pulli primi anni pleyumque in his grifei funt; vnde fpecierum multiplicatio. 
og. Darus 201335 
20. Sternace 1746 
Auis noruegica Kyuffa. Oel.muf. I. $\int$. II. n. 20.

Truen f. Fur. Bartb. act. I. p. 9I.

Coprotheres. Rai. au. 127. n. 2. Martens. Spitz. 63. t. L. f. D.

Auis arçica. Edw. all. 148. t. 148. ct t.149.f. 2.

Habitut intra Tropicun cancri: Europae, Americae, Afiae.

Pifcaturae ineptus agitat congeneres, vomituque eicctuns cibun arripit.

70. STERNA. Roftrum edentulum, fubulatum, rectum, "acutum apïce compreffiúfculo: Nares lineares.

Stolida. I. S. cauda cuneiformi, corpore nigro, fronte albicante. Annoen. Acad. 3. p.

Hirundo marina maior, capite albo. Sloan. iant. I. p. 3I. t. 6. f. 2. Catesb. carol. I. p. 88. t.88.

Anaethetus minor fufcus, vertice cinereo, roftro glabro. Bromn. iant. 481.

Habitat in Americae Pelago.

Pelagicae aues vix bomines aduentantes metumnt.

Hirundo. 2. S. cauda forficata: rectricibus duabus extimis albo nigroque dimidiatis. Fn. fulec. 127 .

Sterna. Gicfn. ak. 53. Aldr. orn. 1. 19. c. 7. Ionft. ail. 130. t. 46 .

Hirundo marina. Will. orn. 268. t.68. Rai. all. 13r. Alb. all. 2. p. 79. t. 90.

Habitat in Europa.

Mas et Fenina indifolubiles focii monogami.

nigra. 3. S. cauda fubforficata, corpore cano, capite roftroque nigro, pedibus rubris. Fin. Jivec. I28.

Hirundo marina minor. Alb. au. 2. p. S2. t. 89.

Sterna fufca. Rai au. I 3 I.

Larus cinereus minor. Aldr. orn...

Habitat in Europa; viuit in Sosictate. 
I 38 AVES ANSERES. Rhynchops.

7r. RHYNCHOPS. Roftrum rectum: mandibula fuperiore multo breuiore.

nigra. I. R. nigra, fubtus alba, roftro bafi rubro.

Rynchopfalia dorfo nigro, ventre albo. Barr. au. 20.

Larus maior, roftro inaequali. Catesb. car. I. p. 90. t. 90.

Plotus roftro conico inaequali. Klein. au. I42.

Habitat in America.

fulve.

fulua.

2. R. fulua, roftro nigro. $f$

Rynchopfalia fulua, roftro nigro. Barr. au. 20. Habitat in America.

Genus proxima afjonitate Laris iunctum. 
7. Rhynchopo. Lenso. 
III a . Wacrorhymchae Liy35.

72. Phoenicoptenuo Liyus.

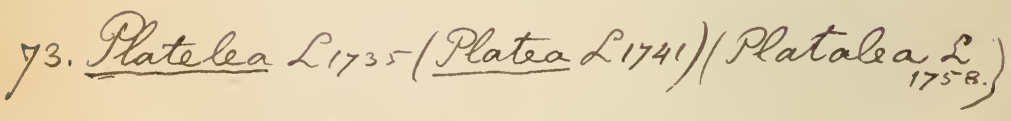




\section{GRALLAE.}

Roftrum fubcylindricum, obtufufculum.

Lingua integra, carnofa.

Femora Supra genuta denudata.

72. PHOENICOPTERVS. Roftrum denudatum, infracto incuruatum. Pedes palmati, tetradactyli.

ruber. I. P. ruber, remigibus primoribus nigris.

Phoenicopterus. Bell. all. l. 8. c. 8. Giefn. alt. . Aldr. orn. 2. l.20.c. 4. Will. orm. 360. t. 60. Rai. au. 117. Tetr. itin. 300. Worm. muf 200. Grew. muf. 67.t. 5. Sloan. iam. 2. p. 32I. AEt. angl. 350 . p. 523. Seb. mulf. I. p. I23.t. 67.f. I. Catesb. car. I. p. 73.t. 73.74. Alb. au. 2. p. 7I. t.77.

Habitat in Africa, America, rarius in Europa.

Dat mibi penna rubens nomen, fed lingur gulofis: Apicio, Caligulae, Vitellio, Heliogabalo expetita fuit, teftantibus Plinio, Martiali, Suetonio, Lampridio.

73. PLA T ALEA. Roftrum planiufculum: apice dilatato, orbiculato, plano. Pedes tetradactyli, femipalmati.

Leuco- I. P. corpore albo.

rodia.

Anas roftro plano, apice dilatato rotundatoque. Fn. fucc. 87.

Platea f. Pelecanus. Gefin. atu. 666. Ionft. ant. ..t. 46. Will. orn. 212. t. 52. Rai. air. 102. Worm. maly: I. $\int .2 . t$. II. f. Iо.

Leucorodias. Aldr. orn. l. 20, c. I3.

Platina f. Cochlearia. Olear: muf. $23 . t$.13. f. 4 .

Alburdiol. Alb. alt. 2. p. 6r. t. 66 .

Habitat in Europa, nidificat in Arboribus.

Roltsuin et Pedes nigri. 
2. P. corpore fanguineo.

Platea incarnata. Sloan. iam. 2. p. 316.

Platea bratilienfis Aiaia dißta. Marcgr. braf. 204. Rai. ail. 102.

ß. Tlauhquechul f. Platea mexicana. Hern.mex. 49. Rai. au. 102.

Habitat in America aufrali.

pygmea. 3. P. corpore fupra fufco, fubtus albo. Muf. Ad. Fr. 2. p...

Habitat Surinami. Magnitudo Paferis.

74. MYCTERIA. Roftrum maxilla fuperiore triquetra rectiffima fubadfcendens, acutum, Maxilla inferiore triNares lineares. gona acuminata adfcendens.

Lingua nulla. Marcgr. Pedes tetradactyli.

america- I. MYCTEZIA.

na. Labiru-guaçu. Marcgr. braf. 20r. Pif. braf. 87. Rai. au. 96. Will. orn. 202.t.47. f.2. 4 .

Habitat in America calidiore.

Magnitudo Ciconiae, alba, remigibus rectricibusque nigro-purpurafcentibus.

Conf. Grew. muS.t. 5.f. I.

75. TANTALVS. Roftrum longum;' fubulatum, obtufum, fubarcuatum, teretiùrculumit.

Saccus iugularis nudus.

Nares ouatae.

Pedes tetradactyli.

Locula- I. TANTALvs. Klein. au. 127.

tor. Pelicanus americanus. Catesb. car. I. p. 81.t. 8I. Tacab, Mife perfis. Chardin. itin. 3. p. 4C. 
yn. Hbycteria Lisso.

Is Tantalues Klein 1950. (Loenlator Hliniss 75 a. Faculator Klein 1750. 
$75 b$. Guns L. 1935

76. Ardea Li735 (Hamiota Kleinisso) 
Habitat in America, Afis.

Tibiae longae. Femora feninuda. Pedes tetrsdacty?i: digitis, 3 anterioribus tantum lafipalnatis.

76. ARDEA. Roftrum rectum, acutum, longum, fubcompreffum, fulco e naribus, verfus apicem exaratum. Pedes tetradactyli.

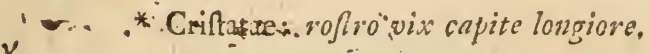

pauoni- I. A. crifta fetofa erecta, temporibus palearibusque binis

กว. nudis.

Grus balearica. Aldr. om. l.20. c.6. Will. om. 20I. t. 48. Rai. all. 95. Dodart. mem. 3.p. I99.

Grus criftata africana. Edw. au. I92. t. I92.

Pauo marinus. Cluf: exot. 105.

Grus capenfis fufca, capite aureo galeato. Pet. gaz. t. $76 . f .9$.

Habitat in Africa.

Caput nigrams. Crifta flauefcens. Alae albae." Cauda nigra aequalis.

Virgo. 2. A. fuperciliis albis retrorfum longe criftatis.

Virgo numidica. Edw. all. 134.t. 134. Dodart. mem. 3.p. I.

Grus numidica. Alb. all. 3. p. 78. t. 83 .

Habitat in Oriente.

Corpus magnitudine Ciconiae caeruleo-cinerenm; capite collo remigibusque primariis nigris. Pennae paiea. res nigrae pendulae. Oculi ribri pupilla cinerea. Pone coulos vitrinque crift a pennacea resuruata longa alba.

* Grues: capite caluo.

canaden- 3. A. fyncipite nudo papillofo, corpore cinereo, alis exfis. $\quad$ tus teftaceis.

Grus fulca canadenlis., Eddw. atu. I33.t.133.

Habitat in America Jiptentrionali.

Grus. 4. A. vertice nudo papillofo, fronte, remigibus oscipiteque nigris, corpore cinereo.

Ardea vertice papillofo. Fn. Jisec. 131. 
Grus. Bell. au. 4r. 6. Gejin. an. 529. Alir. orn. l. 20. c. 5. Ionft. an. I66. t. 44. Will. orn. 200. t. 48. Rai. au. 95. Marj: danub. 5. p.6.t. I. Alb. au. 2. p. 60. t. 65.

Habitat in Europae, Africae campis fubbumidis.

Trans pontun fugat et terris inmittit apricis. Virg.

america- 5. A. vertice temporibusque nudis papillofis, fronte, nucha n3. remigibusque primariis nigris, corpore albo.

Grus americana alba. Catesb.carol. I. p.75.t. 75. Edw. au. 132. t. 132 .

Habitat in America feptentrionali.

Caput jubtus ad maxillam inferiorem rubrum eft."

Antigo- 6. A. capite nudo collarique papillofo, corpore cinereo, ne. remigibus niugris.

Grus maior indica. Edw. an. 45.t. 45 .

Habitat in Afia.

Pone oculos macula farma alba, et vertex albus.

\section{* * Ciconiae.}

Ciconiạ. 7. A: alba, rentigibus nigris, cute fanguineà.

Ardea alba, remigibus rubris. Fn. Jivec. 136.

Ciconia. Bell. au. 45.". Cefn. au. 262. Aldr. orn. 1. 20. c. I. Ionft. au. I47. t. 50. Marjil. danub. p. 26. t. II.

Ciconia alba. Will. orn. 210. Rai. au. 97. Alb. au. 2. p. 5).t. 64 .

Habitat in Europa, Afia, Africa.

Cutis corporis jub pennis junguinea. Nidus ad pagos in altis, rejonans Pafjeribus; buic bonos ferpentum exitio tantus, vt occidere nefas; noctu fridet; migrat trans pontum in Aegyptum, Aetbiopiam; aeftiuat inter. Sueciam et Italiam.

sugra.

ignea. 8. A. nigra, pectore abdomineque albo. Fn. fice. I35.

Ciconia nigra. Gejir. an. 17. Aldr. orn. l. 20. c. t.2.

Will. om. $2 \mathrm{rr}$. Alb. all. 3. p.77. t. 82.

Habitat in Europa boreali.

Migrat neftate jupra Succiam.

****Ardeae.

Nysti- 9. A. crifta nccipitis tripenni dependente; dorfo nigro, corax. $\quad$ abdomine flauefcente. 
76a. Aiconia L.1335/Aaniota Hlém1s50 

Nyeticorax. Gefin. all. 628. Aldr. orn. l. 19. c. 57. p. 272.

Ardea cinerea minor. Will. orn. 304. t. 49. Rai. aw. 99. 17. 3. Mar\%. danub. 5. t. 3. Alb. au. 2. p.62. t.67. Aldr. orn. 1.20. c. 10.

Habitat in Europa anfrali.

Frons alba circum rofirum. Dorfum nigrum, abdonen album. Pennac criflae 3 , teretiufculae, albae, ad nuedium dorji dependentes.

cinerea. Io. A. occipite crifta pendula, dorfo caerulefcente, fubtus albida, pectore m.ıculis oblongis nigris.

Ardea crifta dependente. Fn. Juec. 133.

Ardea cinerea maior. Aldr. orn. l. 20.c.9. Gefin. au. 212. Ionft. au. 151. t. 50. Will. orn. 203. t. 49. Rai. au. 98. Mar.. danub. 5. p. s.t. 2. Alb. au. I. p. 64.t. 67 . et 3. p...t. 78 .

Habitat in Europa; nidificant in arboribus plures $\int i-$ mal.

Herodias. II. A. occipite criftato, dorfo cinereo, femoribus ruffis;, pectore maculis oblongis nigris.

Ard:a fulca canadenfis. Edwo au. 135. $t$. 135 .

Ardea criftara maxima americana. Catesb. carol. 3. $p$. IO. $t$. IO?

Habitat in America.

Tempora alba. Capitis pennae longiores nigrae, vndo poftice criftu euadit. Collum fubtus albido teftaceums fujco maculatum. Brachia antice rufefcentia. Corpus fupra fujcum.

violacea. I2. A. crift flaua, corpore nigro-caerulefcente, fafcia temporali alba.

Ardea ftellaris criftata americana. Catesb. car. I. p. 79. t. 79 .

Habitat in America feptentrionali.

eaerulea. 13. A. occipite criftato, corpore caeruleo.

Ardea maior grifea criftata, capite nigro, vertice et ciliis albicantibus. Brown. iım. 478 .

Ardea caerulea. Catesb. car I. p. 76, t.76. Alb. au. 3. p. 74. t. 79 . Sloan. iam. 2. p. 314. t. $264, f \cdot 5$. Rai. au. 189.

Habitat in America $\int e p t e n t r i o n a l i$. 
friata. I4. A. occipite fubcriftato, dorfo cano firiato, collo fubtus ferrugineo, remigibus fecundariis apice albis. Rolander $r$.

Haubitat Surinami.

Vertex niger nucha fubcriflata. Remiges fufcae, fed ficundariae tuntun margine apicis albae.

virefcens. I5. A. occipite fubcriftato, dorfo viridi, pectore rufeficente.

Ardea frellaris minor. Sloan. iam. 3. p. 3I5. t. 263. Rai.au. 189. n.2. Catesb. cav. I. p. 80.t. 80.

Habitat in America.

frellaris. I6. A. capite laeuiufculo, fupra teftacea maculis transuerfis, fubtus pallidior maculis oblongis fuf́cis.

Ardea vertice nigro, pectore pallido maculis longitudinalibus nigricantibus. Fin. füec. 134 .

Ardea ftellaris minor. Gefin. an. 215. Aldr. om. l. 20. c. 16. Will. orn. 207. t. 50. 52. Rai. au. I00. n. II. Marf. danub. 5.p. I6. t. 6. Alb. an. I. p. 65. t. 68 .

Habitat in Europa.

Venatus falconibus magnatibus placet; Mugitus ftenturii impares; terrefacta Spiraliter aetbera jcandit.

2liba. I7. A. capite laeui, corpore albo, roftro rubro. Ardea tota alba, capite laeui. Fn. Jikec. 132. Ardea alba maior. Will. orn. 205. t. 43. Rai. all. 99. n. 4 .

Habitat in Europa.

Ibis. I8. A. capite laeui, corpore albo, roftro flauefcente apice pedibusque nigris.

Ardea Ibis tota alba, pedibus atris, vnguibus arcuatis maximis. Hafjelqu. irin. 248.

B. Ibis nigra. Rai. au. 93.

Habitat in Aegypto; regionis purificator.

Bellonii Ibis roftro arcuato, nigra eft; at Plinins $X$ : 30 :

Ibis circa Pelufinu tantunn nigra eft, cacteris omibus locis candida.

aequino- 19. A. capite laeui, corpore albo, remigibus duabus priatialis. mis margine exteriore fufcis. Rolander. Habitat in America. 

IV. Lolopaces, Liyss / Grallar Lisss.

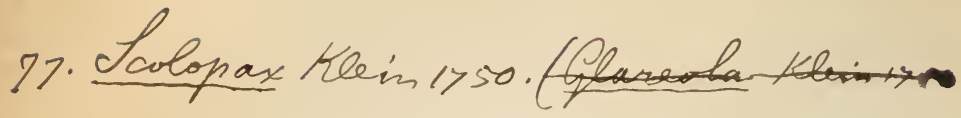

Tya. Namenins Ler35. 
s-a- . Sinillima Ibidi tota flructura; at vero Resgiges primores omnes apice firficforntes, et I-2 etiann margine exteriore longitudinali fufcae, caeterum cadem, it facile pro varietate agnojcatur.

77. SCOLOPAX. Rofrum teretiufculum, obtufum, capite longius.

Pedes terradactyli: poftico pluribus articulis infiftente.

tubra. I. S. roftro arcuato, pedibus rubris, corpore fanguineo, alarum apicibus nigris.

Porphyrio amboinenfis f. Ardea rubra corallina. Seb. minj: I. p. 98. t. 62.f. 3 .

Numenius ruber. Catesb. car. r. p. 84.t. 84.

Numenius indicus. Cluf: exut. 366.

Guara, Marcgr. braf. 203. Will. orn. 239. Rai. au. 104. 17.6.

Habitat in America.

alba. 2. S. roftro arcuato, pedibus rubris, corpore albo, alarum apicibus viridibus.

Numenius albus. Catesb. car. I. p.32. t. 82 .

Habitat in America.

fufca. $\quad 3$. S. roftro arcuato, pedibus rubris, corpore fufco, cauda bali alba.

Numenius fufcus. Catcsb.car. r.p. 83.t. 83 .

Habitat in America.

Antecedentes I. 2.3. frutura nimis affines, fed colore corporis diucrjac.

Totanus. 4. S. roftro reito bafi rubro, pedibus coccineis, remigibus fecundariis albis. Frr. fuic. I 49 .

Totanus. Gifse. att. 51 S. Aldr. orn. l. 20. c. 24. Will. onn.220.t.53.f.4. Rai.an.107. All.atl.3.p.S2. t. 87.

Habitat in Europa.

Arquata. 5. S. rofiro arcuato, pediluis caerulefcentibus, alis nigris maculis niueis. Fin. fuec. 139.

\section{K 3}

Nume- 
Numenius f. Arquata. Gefin. au. 222. Aldr. orn. I. 20. c. 21. Will. orn. 216. t. 54.f. I. 2. Rai. au. 103. Alb. au. 1. p. 75. t. 79. Marf. danub. . . t. 17.

Habitat in Europa.

Phaeopus.6. S. roftro arcuato, pedibus caerulefcentibus, maculis dorfalibus fufcis rhomboidalibus. Fn. fuec. 140.

Phaeopus altera f. Arquata minor. Gefr. au. 500. Aldr. orn. l.20. C. 21 .

Arquata minor noftras. Rai.au.103.n.2. Will.orn. 217.

Habitat in Europa.

Surfun recta enolans clamando tempeftates praenuncians.

Ruftico- 7. S. roftro recto laeui, pedibus cinereis, femoribus tectis, la. fafcia frontis nigra.

Numenius roftri apice laeui, capite linea vtrinque nigra, rectricibus nigris apice albis. Fn. fuec. I4I.

Scolopax f. Perdix ruftica. Gefn. au. 502.

Scolopax. Aldr. orn. l.20. c.5I. Will. orn.213. Rai. air. 104.n. 1. Alb. au. 1. p.67.t.70.

Habitot in Europa.

Noctu volitat reditque itcruto eadem via aërea.

Fedoa. 8. S. roftro recto longo, pedibus fufcis, remigibus fecundariis rufis nigro punctatis.

Fedoa americana. Edw. au. $137 \cdot t .137$.

Habitat in America Jeptentrionali.

Corpus grifeum. Remiges primores fufcae. Linea fuperciliorum alba.

Glottis. 9. S. roftro recto bafi inferiori rubro, pedibus virefcentibus.

Numenius pedibus virefcentibus, vropygio albo, remigibus lineis albis fufcisque vndulatis. Faur. Juec. 142.

Pluuialis maior. Aldr. orn. l.20. c. 66. W'ill. orn. 220. t. 55. Rai. a16. 106.

Glortis. Gejn. au. 520 .

Habitat in Europa.

10. S.

Species Scolopacum, Tringarum etc. difficillime diftingunntur, aetate fexuque matabiles, colore parum diftinctae; quae pedibus funt rubris a reliquis probe ob/eruandae, сиm pedes non varient. 


Limofa. Io. S. roftro ręto laeui, pedibus fufcis, remigibus macula alba: quatuor primis immaculatis.

- Numenius vropyg1o albo, rectricibus nigris bafi albis, remigibus transuerfa alba macula; exceptis quatuor primis. In. fuec. 144.

Habitat in Europa.

Gallina- II. S. roftro recto apice tuberculato, pedibus fufcis, lineis go. frontis fufcis quaternis.

Numenius capite lineis 4. fufcis longitudinalibus, roftri apice tuberculofo, femoribus feminudis. Faun. filec. 143 .

Gallinago minor. Gejn. ant. 503. Aldr. orn. 1.20.c. 53. Will. orn. 214. Rai. au. 105. 21.2. Alb. au. 1. p. $68 . t .7 \mathrm{I}$.

Hubitat in Europa.

Haec Capella caeleftis, cuius mus tam alte celerrimeque volitat, vt audiri, non vero oculis attingi poffit.

lapponi- 12. S. roftro fubrecuruato, pedibusque nigris, pectore fer$\mathrm{ca}$. rugineo.

Recuruiroftra pectore croceo. Fn. Juec. 138 .

Habitat in Lapponia. Rudbeck.

Aegoce- 13. S. roftro ręto, pedibus virefcentibus, capite colloque phila. rufefcentibus, remigibus tribus nigris bafi albis.

Barge f. Aegocephalus. Will. orn.215. Rai. au. I05. n. 4. Alb. au.2.p.64. t. 70.

Habitat in Europa auftrali.

Haema- I4. S. roftro refto flauefcente, pedibus fufcis, corpore fubftica. tus fuluo furco - vndato.

Fedoa americana, pectore rufo. $E d w$. au. 138. t. 138. Habitat in America Jeptentrionali.

Corpus grijeum Jubtus rubro - fuluefcens.

Rectrices primores nigrae apice albas.

Scolopaces digitis quatuor infiftune, per paludes vadant, Tringac percamspos littorague currunt vix digito poffico infiftantes, vti rite Cel. Kileiniиs notanit. 
78. TRINGA. Rofrmm teretiufculum; . longitudine

$$
\begin{aligned}
& \text { capitis. } \\
& \text { Pedes teradactyli: poftico vniarticu- } \\
& \text {. lato a terita eletato. }
\end{aligned}
$$

Pugnax. 1. T. pedibus rubris, teetricibus tribus lateralibus immacularis, facie papillis granulatis carneis. Fir. furec. I45. Auis pugnax. Aldr. orn. l. 20. c. 19. f. 1-8. Ionft. aum. 152. t. 51. 52. Will. orn. 224. t. 56. Rai. an. 107. Marf. damlb. 52. t. 24. Alb. au. I. p.69.t. 72.73. Habitat in Europa mimus boreali.

Mares diffiniles, pugnaciffini.

Vanel- 2. T. pedibus rubris, crifta dependente, pectore nigro. lus. Fin. firec. 148 .

Capella f. Vanellus. Bell. an. 49. a. Gefn. au. 764 . Aldr. orm. l. 20. c.63. Will. orm. 228.t.57. Ionft. au. I64. t.53.27. Rai. au. I10. Alb. au. I. p. 70. t. 74 :

Habitat in Europa, Africa.

Migrat forte in Acgyptum. Oun Belgis in deliciis.

Gambet- 3. T. roftro pedibusque rubris, corpore luteo cinereoque ta. variegato, fubtus albo. Fn. fitec. 153 .

Gambetta. Aldr. orr. 1.20.6.26. Rai. als. I07. Habiatat in Europa.

Interpres. 4. T. pedibus rubris, corpore nigro albo ferrugineoque vario, pectore abdomineque albo. It. got i. 217. Fir. fuec. 154 .

Morinellus canadenfis, Echvo, aul. I4I. t. I4I. Habiat in Europa et America feptentrionali.

lobata. 5. T. roftro fubulato apice infexo, pedibus virefcentibus lobxts, abdomine albido.

Tringa fufca, pedibus fulicae. $E d w$. au. I43. t. I43. et $46 . t .46$.

Habitat in America Septentrionali, Lapponia.

Roltrum temuif:mun eft; procellojà tempeftate natat gregaria.

Fulicaria. 6. T. roftro recto, pedibus lobatis fubfufcis, abdomine ferruginco. 
90 Tringa L1735.

7sa. Vanellus, L1735

79 a. Glareola Klein 1750. 
Giaresla Heim nss. 
Tringa fufca canadenfis, membranis ad digitorum articulos. Edwo. an. I42. $t$. $\mathrm{I}_{4} 2$.

Habitat in Americi.

alpina. 7. T. teftaceo - fufca, pectore nigricante, rectricibus cinereo-albidis, pedibus fufcefcentibus.

Habitat in Lapponia. Adlerbcim.

Magnitudo Sturni; Abdomen albunn.

Ocro- \&. Tringa roftri apice punftato, pedibus virefcentibus, dorphus. fo fufco-viridi nitido. Fn. Juec. 146.

Tringa. Aldr. orn.l.20.c. 54. Will. orn. 222. t. $55^{\circ}$ Rai. au. IoS.

Rhodophus f. Ocrophus. Giefn. au. 508.5II. Aldr.om. l. 20 . c. 39 .

Habitat in Europa.

Hypoleu- 9. T. roftro laeui, pedibus liuidis, corpore cinereo lituris cos. nigris: fubtus albo. Fin. Jizec. 147.

Tringa minor. Will. orm. 223.t. 35. Rai. au. 108 .

Gallinula hypoleucos. Giejir. au. 509. Aldr. orm. 3. p. 469 .

Habitat in Europa.

Canutus. IO. T. roftro laeui, pedibus cinerafcentibus, remigibus primoribus ferratis.

Tringa cinerea, remigibus fecundariis bafi albis, rectricibus quatuor mediis immaculatis. Faun. Jive. 150. Canuti auis. Rai. au. Ios.

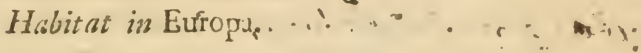

Glareo- Ir. T. roftro laeui, pedibus virefcentibus, corpore albo la. punctato, pętore fubalbido. Iin. Jisec. 152. Habitat in Europa.

littorea. 12. T. roftro lacui, pedibus fufcis, remigibus fufcis: rachi primae niuea. Im. Juec. 15 I.

Habitut in Europa.

Squata- I3. T. roftro nigro, pedibus virefcentibus, eorpore grifeo rola. fubtus albido. Fin. Jivec. 155 .

Pluuialis cinerea. Rai. au. IIr. Alb. au. I. p. 72. t. 76.

Habitat in Europa. 

79. CHARADRIVS. Roftrum, teretiufculum, obtuPedes tridactyti.

criftatus. I. C. gula, pileo'pectoreque nigris, occipite criftato, dorfo teftaceo, pedibus nigris.

Pluuialis indicus, pectore nigro. Edwo. au. $47 . t .47$. Habitat in Perfia.

Simillinus eft Tringae Vanello.

Hiaticu- 2. C. pectore nigro, fronte nigricante fafciola alba, verla. tice fufco, pedibus luteis. Fn. fuec. I59.

Charadrios f. Hiaticula. Aldr. orn. l. 20. c.67. Ionft. au. 166. t. 53. Will. orn. 230. t. 57. Rai. au. 120. Sloan. ian. 2. p. 314. t. 169. f. 2. Alb. au. I. p. 76. t. 80.

Habitat in Europa et America ad ripas.

alexan- 3. C. fufcus, fronte collarique dorfali abdomineque albis, drinus. rectricibus lateralibus vtrinque candidis, pedibus nigris. Charadrius alexandrinus. Haffelq. iter. 255. n. 30. Habitat ad Aegypti ex Nilo canalem.

vocife- 4. C. fafciis pectoris colli frontis genarumque nigris, caurus. da lutea fafcia nigra, pedibus pallidis. Pluuialis vociferus. Catesb. car. I. p.7I. t. 7 I. Habitat in America Septentrionali.

aegyptius. 5. C. fafcia pectorali nigra, fuperciliis albis, reßtricibus apice allis fafcia nigra, pedibus caeruleis.

Charadrius aegyptius. Hafjelq. iter. 256.n. 31. Habitat in Aegypti apricis.

Morinel- 6. C. peetore ferrugineo, fafcia fuperciliorum peetorisque lus. lineari alba, pedibus nigris. Fn. Juec. 158.160. Morinellus. Alb. au. 2.p. 57.t.6r. 62. Rai. au. IIr. Habitat in Europa: ad Alpes migrans per Vp aliam medio Maii feptentrionem ver [us.

Mas ad frobiculum abdominis niger, femina non.

aprica- 7. C. pectore nigro, roftro bafi gibbo, pedibus cinereis. rius. Charadrius nigro-lutefcente variegatus, pectore nigro. Fn. Juec. 156 . Iter. oel. 72 . 


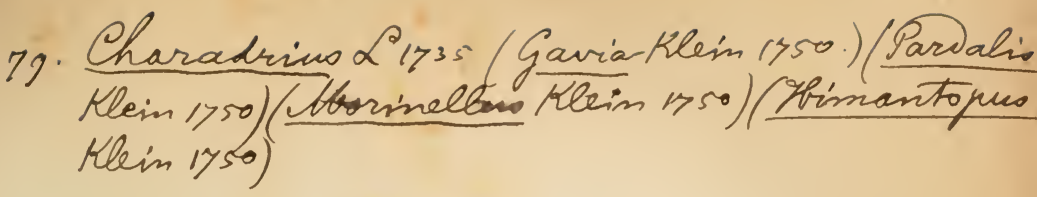


0. Recurvirostra $\alpha_{1746 .}$ 
Pluuialis viridis maculatus, ventre nigro. Edrp. au. 140. $t$. 14 O O.

Habitat in Oelandia, Canada.

Pluúialis. 8. C. pedibus cinereis, corpore nigro viridique maculato, fubtus albido. Fin. fitec. 157 .

Pluuialis viridis. Will. orn. 229, $t .57$. Rai. all. IIr. Sionis. ium. 2. p. I13.t. 269. f. I. Alb. alt. I. p. 7I. t. 75 .

Havitat in Europa.

Oedicne- 9. C. grifeus, remigibus primoribus duabus nigris medio mus. albis, roftro acuto, pedibus cinereis.

Oedicnemus. Bell. ali. . Rari. all. 108. no. 4. Alb. ali. I. p. 66.t. 69.

Charadrius. Ciefn. au. 256 .

Charadrius Keruari. Hafjelq. iter.210. n.32. an?

Habitat in Anglia.

Himan- Io. C. fupra niger, fubtus albus, roftro nigro capite lontopus. giore, pedibus rubris longiffimis.

Charadrius autumnalis. Haffelq. iter. 253. n.29.

Himantopus. Sibb. fcot...t. II. 13. Rai. au. 106. Aldr. orn. 1. 20. c. 30 . Giefin. au. ..

Habitat in Europa auflraliore.

fpinofus. II. C. remigibus, pectore, abdomine pedibusque atris, teEtricibus dimidiato-albis, humeris fpinofis.

Charadrius fpinofus. Haffelq. itin. 260.270.

Habitat in Aegypto.

Humeri jpinofi, vt in Fulica Jpinofa, fungulares funt.

8o.RECVRVIROSTRA. Roftrum depreflo-planum, dcuminatum, recuruatum. Pedes palmati, terradactyli.

Auofet- I. R. albo nigroque varia. It. ocl. 89. Fn. fuec. I37.

ta. Auofetra f. Recuruiroftra. Gejin. als. 232 . Alds.orn. l.19. c. 64. Will. orn. 240. t. 60. Rai. all. 117. Marj: danub. 5. p. 72.t. 34. Alb. au. I. p. 96. t. 101. Habitat in Europs anflraliore.

Migrat in Italiam, rariffima apud nos. 
Sr. HAEMATOPVS. Rofrum compreffum: apice cuneo aequali. Pedes ividactyli, firi.

Oftale. I. HA EMATOPVS. It. oel. 83. Fn. Juec. 161. Bell. au. gus. IS. Aldr. orn. l.20. c. 35. Will. orn.220. t. 56. Rai.au. 105. Alb. all. I. p.74. t.78. Catesb.car. I. p. $85 . t .85$.

Habitat in Europae, Americae feptentrionalis littoribus marinis; victitat conclis.

82. FVLICA. Roftrim conuexum: Mandibula fuperiore margine fupra inferiorem fornicata; Mandibula inferior pone apicem gibba.

Frons calua.

Pedes tetradactyli, fublobati.

atra. I. F. fronte calua, corpore nigro, digitis lobatis.

Fulica fronte calua aequali. Fn. Juec. 130.

Fulica. Bell. atu. 36. b. Gejn. au. 390. Aldr, on.7. I9. c. I3. Will. ornitb. 239. t. 59. Rai. au. II6. Marfil. damb. 70. t. 33. Alb. au. I. p. 79. t. 83. Haffelq. act. $v p$. $175 \mathrm{I} \cdot$ p. 22. itin. 262.

Habitat in Europa; bybernat in Gallia.

Edit Sernina, Herbas; natut fupraque aquam currit.

Chloro- 2. F. fronte calua, corpore nigro, digitis fimplicibus. pus.

Gallinula Chloropus. Alb. au. 2. p. 66. t. 72. et 3 . p. 86.t.9I. Rai. au. II3.

\section{Habitat in Europa.}

Porphy- 3. F. fronte calua, corpore violaceo, digitis fimplicibus. rio.

Fulica maior pulla, fronte cera coccinea oblongo-quadrata. Brown. iam. 479.

Porphyrio. Alb. au. 3.p. 79. t. 84. Rai. au. 116.n. 13. Edwo. alt. 87.t. 87. Dodart. at: 3. p. 30? Habitat in Alia, America.

ipinofa. 4. F. fronte carunculata, corpore variegato, humeris fpinofis, digitis fmplicibus, vngue poftico longifimo. 
81. Buematopus. Lin35 (OStrarlega Klem 1950.

02. Fulica L. pyss. 
02a. Ortygometra Lavab,
0.3. Rallus Xlein 1y50. 
Gallinula alis cornubus donatis. Edw. au. 48 . t. 48 . Habitat in America auflrali.

Vnguis pofticus recius, digito longirr. Pollex znius ar. ticuli infillens. Renniges virides.

83. RALLVS. Rofrum bafi craffus, compreflum, dorfọ attenuatum apicem verfus, aequale, acutum.

Pedes terradactyli, fiffi. Corpus comprefium.

Crex. I. R. alis rufo-ferrugineis.

Ortygometra alis rufo-ferrugineis. Fn. fuec. I62.

Ortygometra. Aldr. urm. l. I3.c.23. Will. om. 522. t. 29. Rai. all. 58. Alb. au. I. p.30.t. 32.

Habitat in Europae agris, carectis; victitat Lunbricis.

Corpus walde comprefJum; vefperi et nocte fonorus voce ingeninata crex.

aquaticus.

2. R. alis grifeis fufco-maculatis, hypochondriis albo-maculatis, roftro luteo.

Rallus aquaticus. Alb. al. I. p.73. t.77. Will. orn. 234. Aldr. orn... Rai. au. 113.n. 2.

Ortygometra alis fufcis. Syft. nat. 25.

Habitat in Europa.

larifor- 3. R. fubtus albido-flauefcens, ceruice caerulefcenti mamis. culato, digitis marginatis. $f$

Larus fiffipes. Alp. au. 2. p. 75.t. S2.

Rallus cinereus facie Lari. Klein. an. 103.

Hiabitat in Europa.

Cauda buic longiufcula; ideoque anceps genus.

bengha- 4. R. corpore vertice oculisque albis, capite colloque nilentis. gris, alis dorfoque viridibus, remigibus primariis rubro-maculatis.

Rallus benghalenfis. Alb. aw. 3. p. 85 .

Habitat in Afia.

carolinus, 5. R. grifea, fronte nigra, pectore plumbeo, rofto flauo, pedibus virefcentibus. 
Gallinula minor canadenfis. EdiD. au. 144. t. 100. Catesb. car. I. p. 70.t.70.

Habitat in America feptentrionali.

84. PSOPHIA. Rofrum cylindrico-conicum, conuexum, acutiufculum, mándibula fuperiore longiore. Nares ouatae patulae.

crepitans. I. PSOPH I A.

Pfophia crepitans nigra, pectore columbino. Barr. au. 62. Gallina fyluatica crepitans, pectore columbino. Barr. aequin. 132.

Gallina fylueftris Macucagua. Rai. au. 53 .

Macucagua. Marcgr. braf 213. Pif. braf.88.

Habitat in America meridionali.

Roftro mufitans rejpondet per anum.

85. OTIS. Roffrum mandibula fuperiore fornicata. Lingura bifida. Pedes tridactyli absque poftico.

Tarda. I. O. iugulo vtrinque criftato.

Tarda. Dodart. mem.3.p.309. Edw.au.73.t.73.74. Alb. au. 3. p. 36. t. 38. 39 .

Otis f. Tarda auis. Rai. au. 38. Aldr. orn. l. 13. c. 12. $E d w$. แu. 73. t. 73 .

Habitat in Poloniae, Orientis campis; migrat per Belgium, Anglian. Menjis eximia.

arabs. 2. O. auribus eręto-criftatis.

Otis arabica. Edwo. au. 13.t.12.

Habitat in Oriente.

Supra folopacina, fubtus albida. Aures nigrae. Cauda fafcia fufca.

Tetrax. 3. O. capite iuguloque laeui.

Anas campeltris. Alb. au. 3. p. 33. t. 4I. Rai. ail. 59 .

Tetrax. Bell. au. .. Aldr. orn. 1. 13. c. I3.

Habitat in Europa, imprimis in Gallia. 
04 Poophia L 1750.

85 Otis L1735 ( Farva Klein 1750. 
06. Ltuntio L1735 (Dturtio Livas)

o6a. Casuarins L1y35. (Casearius Kleinsy50) 
afra.

4. O. nigra, dorfo cinereo, auribus albis. Habitat in Aethiopia. I. Lizmannus.

Maris Roflruni et Pedes fiaui. Vertex cinereus. Alarum margo exterive aibus. Femina tota cinerea, exceptis femoribus abdonineque atris.

86. STRVTHIO. Rejtmum conicum.

Alare ad volandum ineptae.

Camelus. I. S. pedibus didactylis.

Struthio-Camelus. Aldr. orn. l. 9.c. 2. Dodart. mem. 335. Seb. mul. x. t. 5I. f. 6. Will. ornitb. 104. t. 5 . Rai. all. 36. Alb. all. 3. p. 30. t. 3I.

Habitat in Syria, Arabia, Lybia, Africa.

Spina Jüb bumeris armatur, maxima Azium; Palpebra vtraque ciliata. Exterior digitus muticus. Pennae lanatae pro galeis bellicis criftaque caftrenji. Outa jaepe 50 , candida, laeuia, in arena excludenda deferta. Iob.39: 16.

Cafuarius. 2. S. pedibus tridactylis, vertice palearibusque nudis.

Cafuarius. Olear. 'mul:" 23. t. 13. $f .2$. Alb. al. 2. p. 56.t. 60.

Emeu. Dodurt. mem. 377. Rai. att.36. Cluf. exot. 98. Bont. ial. 71. Will. ornith. 105. t. 25. Worm. mut... Ionft. au. 172. t. $56 . \quad$ Aldr. 0rit. 1. 20. t. $54 \mathrm{I}$.

Habitat in Afia, Sumatra, Molucca, Banda.

Callus eleuatus quar: diadema verticis. Palearia 2 fub collo; Collum a tergo nudum. Vugate intermedio maiore fort ; our punctis excisuatis.

america- 3. S. digiro poftico rotundato mutico.

nus. Seruthio-Camelus americanus. Rai. an.36.

Nhanduguacu bratilientibus. Marcgr. braf: syo. Pif. braf. 84 .

Habitat in America auffrali.

cuculla- 4. S. pedibus tetradaetylis, omnibus unguiculatis.

tus. Gallus gallinaceus peregrinus. Cluf. exut. 99. t. 100. Olear. mul: $23, t \cdot 13 \cdot f \cdot 5$.

Cygnus cucullatus. Nicremb. nat. 23I. Rai. as. 37.

Dronte. Bont. ian. 70.

Habitat in India. 


\section{$V$. GALLINAE.}

Rofrum conuexum: mandibulat fuperiore fornicata, margine exira inferioren dilatato.

Nares mombrana cartilaginea comexa femitectae.

87. PAVO. Caput pennis rectis reuolutis teetum. Perznae dorfales elongatae.

criftatus. I. P. capite crifta erecta.

Pauo. Gefn. all. 656. Aldir. orn. l. I3. c. I. Ionf?. all. 56. t.22. Will. om. II2. t.27. Rai. all. 5I.

Habitat in India orientali, Zeylona.

Mas remigibus primoribus ferrugineis, eiulans, vindicans, Juperbus, togann doryalem gennmantibus ocellis, naturae mivuculo pictan erigendamque, tertio amo acquirit, qua nibil pulcbrius, fed canicula deiicit. Fenina curat pullos in proximas muptias. Sanbuci floribus occiditur.

bicalca- 2. P. capite laeui, calcaribus duobus.

ratus. Phafianus pauonicus chinenfis. Edw. alt.67.t.67. et t. 69. f. $\mathrm{r}$.

Habitat in China.

Pennae verticis rewolutae. Pennae pofterioris dorja longae ocellis.

88. MELEAGRIS. Caput carunctilis fpongiofis tectum.

Gallo- I. M. capite caruncula frontali, criftaque gulari, maris pepaùo. . Etore barbato.

Meleagris. Fn. Juec. 164.

Gallo-pauo fylueftris nouae angliae. Rai. au. 5r. Alb. au. 3. p. 33. t. 35 .

B. Gallopauo. Gefir. au. 482. Aldr. ornith. l. 13. c. 4. Bell. an. 60. a. Ionft. aw. 58.t.24. Will. orn.II3. t. 27. Rai. $333.5 \mathrm{I}$. 
V. Gallinae Lins

0). Pavo Loy3s.

son tholeagris 201735 
og Prax 21740. 
\%. Gallopauo criftatus. Alb. all. 2. p. 30. t. 33.

Habitat in America Septentrionali.

Mas exaeltuat inflato pectore, expanfa carda, fanguinea facie, relaxata frostis caruncula: irae tenax; Sapida caro. Rectrices XVII. Barba pectoris tertio anno excrefcit.

criftata. 2. M. capite pennis erectis criftato, temporibus violaceis.

Quan f. Guan. Edw. all. 13.t.13.

Iacupema: Marcgr.braf: 198.

Coxolitli. Hern. mex...

Phalianus bratilienfis. Rai. au. 59.

Habitat in Americi auffrali.

Gula caruncula compreffa rubra, vt in antecédente, ca. pite fupra crift a oblonga pennacea.

Satyra. 3. M. capite cornubus duobus, corpore rubro punctis nigris albo foetis. $\dagger$

Phatianus cornutus. Edw. II6. t. II6.

Habitat in Benghala.

Frons nigra. Caruncula gularis caerulea rufo variegata.

Cornua duo caerulea pone oculos enata.

89. CRAX. Roftrum bafi cera obtectum.

Pernac caput tegentes reuolutae.

nigra. I. C. nigra. Syft. nat. 27.

Gallus indicus. Sloan. iam. 2.p.302. t. 260. Dodart. mem. 146 .

Gallus indicus alius. Aldr. orn. . .

Mita f. Mutu. Will. orn. . t. 28. Rai. al. 52. Marcgr. braf. 194. 195.

Gallus curaffauicus. Aldr. au. 2. p. 29.t. 3 1. 32 .

Habitat in America auftrali.

rubra. 2. C. rubra, capite caerulefcente. $†$

Gallina peruuiana rubra. Alb. au. 3. p. 37. t. 40.

Habitat in America.

An :arietas praccedentis? 


\section{PHASI A V V S. Gcrac cute nuda laeuigata.}

Gallus. I. P. cauda compreffa adfcendente. Fn. Juec, 165.

Gallus gallinaceus f. Gallina. Gefir. au. $395 \cdot 340$. Aldr. orn. l. I4.c. I. Will. orn. 109. t. 26. Rai. au. 5I. criffatus. $\beta$. Gallina crifa in vertice plumofa denfifima. Rai. ati. 5 I.

ecaudatus. $\gamma$. Gallina cauda f. vropygio carens. Rai. au. 5 I. crijpus. J. Gallina frislandica. Will. orn. 1ro.

Gallina crifpa. Rai. an. 51. Aldr. orn. l. I4. p. 329.

puffllus. E. Gallus tibiis pennatis.

Gallina bentamenfis. Alb. au. 3.p. 33.t.33. 34 .

Habitat in Incia Orientali: Poulicandor, etc.

Gallus pugnax, Juperbus ceifa crifta, evecta falcataque canda, vigil, plaudentibns lateribus canens gallicinia, falax 10. feminis firficit. Gallina pulueratrin oua copiofa ponit, ravo fubuentanea, nifi Capis afjociata: cibis expetita oua, caro, iufcula galli jenioris. Laeditur baccis Sambuci; a pedicalis liberatur pipere.

Melez- 2. P. vertice callofo, temporibus carunculatis. gris.
Gallina vertice comeo. Haffelqu. iter. 274.,
Gallus vertice corneo. Syjt. nat. 28.
Gallina guinea. Worm. mus. 297.
Gallina africana. Maregr braf. 78 .

Gallus niger, maculis oualibus et orbiculatis. Barr. au. 79.*

Gallina guineenfis. Worm. mus. 297. 297. Oleas. mul: .t. 15. f. 3. Rai. au. 52. Alb. au. 2. p. 32 . t. 35. Will. ern. 562. t. 26. Aldr. au. l. I4. c. 13. Hebitat in Africa.

colchi- 3. P. rufus, capite caeruleo.

cus.

Phafianus. Kai. au 56. Alb. au. I. p. 24. t.25. 26. Olin. au. 49. Aldr. orm. l.13. c.5.

Habitat in Africa, Afis.

Principum menjis dicatus.

pistus. 4. P. crifta flaua, petrore coccineo, remigibus fecundariis cacruleis. Annoen acad. I. p. 282. t. 13 .

Phafianus variis coloribus fplendidus. Edw. an. 68.t.68. ct t.69. f. 2 .

Phalianus ruber, e china. Alb.au. $3 \cdot p \cdot 34 \cdot t \cdot 36$.

Habitat in China. 
91. Setrao $\alpha_{1435}$

q1.a. Lagopus Klem 1750. 
nycthe- 5. P. albus, crifta abdomineque nigris.

merus. Phafianus linenfis albus è nigro caeruleus. Alb. al\% 3 . p: 35. t. 37. Ed: all. 66. t. 66.

Habltat in China.

Tempora nuda rubra. Maris crifta nigra. Femina grijéa eft.

91. TETRAO. Supercilia nuda, papillofa.

* Pedibus birfutis.

Vrogal- I. T. pedibus hirfutis, ręricibus exterioribus fubbreuiorilus. bus, axillis albis. Fin. Jikec. 166.

Vrogallus f. Tetrao maior. Gejn. atr. 491. Aldr. orr.

l. 13. c. 6. Will. orn. 123.t.30. Rai. au. 53 .

Mufcouian. Aib. all.2. p.28.t.29. 30.

Habitat in Europae frigidae pinetis paindofis.

Exaeftuat in arboribus, efflans timnitu tanquam ecftafi corripitur.

Tetrix. 2. T. pedibus hirfutis, cauda bifurcata, remigibus fecundaris bafin verfus albis. Fin. ficec. 163 .

Tetrao f. Vrogallus minor. Gefin. all. 494. Aldr, orn. l.c. 7. Will. orn. 124. t. 31. Rai. au. 53. Alb. au. I. p. 22.t. 22.

Habitat in Europae frigidae Ericetis, Betuletis.

Exaeftuat in campis obmurmurans erecia cauda tumidisque finperciliis, fibilo efllans; bieme jaepe niue Jepelitur; out exchulens odore priuater.

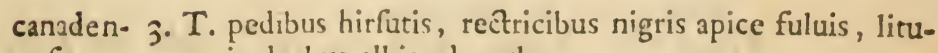
fis. ris duabus albis ad oculos.

Vrogellus maculatus canadenfis. Edw. au. II8. t. II8. mas. et 71. t. 71. fimina.

Habitat in Canada.

Lagopus. 4. $T$. pedibus lanatis, remigibus albis, reftricibus nigris apice albis: intermediis totis albis.

Tetrao rectricibus albis: intermediis nigris apice albis Fn. pince. 169 .

Lagopus. Gefin. all. 577. Aldr. orn. 1. 13. c. 21. Will. orn. 127. t. 32. Rai. au. 55. Edtr. all. 72. t. 72 . Habifat in Europae alpinis, ex Betula nana viefituns. 
Pedibus lanatis leporis; byene albus exceptis rectricibus, fub niue faepius cuniculos agens; in filuis loquax cacbinnis.

Phafia- 5. T. pedibus hirfutis, cauda cuneiformi, rectricibus tribus nelius. lateralibus albis.

Vrogallus minor, cauda longiore. Edw. an. II7. t. II7. Habitat in Canada.

Cupido. 6. T. pedibus hirfutis, alis fuccenturiatis ceruicalibus.

Vrogallus minor fufcus, ceruice plumis alas imitantibus donata. Catesb. car. 3.p. I. t.I.

Habitat in Virginia.

Color Tetricis feminae; Vertex fubcriftatus; a tergo colli duae paruac alae: fingulae ponnis quinque.

Confr. Phafianus Penfyluanicus. Act. angl. 48 . p. 499 . t. I5.

Bonafia. 7.T. pedibus hirfutis, rętricibus cinereis pundtis nigris fafcia nigra: exceptis intermediis duabus. Fn. Juec. I70.

Gallina corylorum. Gefin. alt. 229. Aldr. orn. 1. 13. c. IL. Will. orn. 126.t.21. Rai. au. 55.

Habitat in Europae Coryletis.

Fiftula dulce canit volucrem dum decipit auceps.

** Pedibus nudis.

iufus. 8. T. roftro pedibusque fanguineis, gula alba cincta fafcis nigra albo punctata.

Tetrao rectricibus cinereis: fuperiore tnedietate hinc inde rufis. Fn. Jiuec. I7 I.

Perdix rufa f. maior. Gefn. au. 682. Aldr. orn. l. 13. c. 18. Ionft. au. 68. t. 27. Will. orn. 118. t. 29. Rai. au. 57. Alb. au. 1. p.29. t.29.

Habitat in Europa anftrali.

Perdix. 9. T. pedibus nudis, macula nuda coccinea pone oculos, cauda ferruginea, fterno brunneo. Fn. fivec. 172.

Perdix cinerea. Aldn 01m. l. I3. c. I9. Ionft. an. 68. t. 27. f. 1. Will. orn. II9. t. 29. Rai. au. 57. Alb. au. $l$ p. $25 . t .27$.

Habitat in Europae agris.

Hyeme babitat intra foljas niuis vtraqua extremitate apertas. 
11.6. Percix Menn 1900. 
91c. Coturnix. Kleín vso. 
virginia- 10. $T$. pedibus nudis, fufcia nigra fupra et infra oculos, nus. linea verticali fulua.

Perdix virginiana. Catesb.car.3.p. 12.t.12. Habitat in America.

marilan- II. T. pedibus nudis, ceruice albo nigroque pun\&tata.

dicus. Perdix nouae angliae. Alb. au. I. p. 26. t. 28.

Habitat in America.

In Perdice rufa funt puncta alba in difco nigro infra guttur verjus pectus, in bac vero eiusmodi a tergo colli, nec pedes rubri.

orienta- 12. T. pedibus antice pilofis: abdomine gulaque atra, collis. lari ferrugineo, cauda cuneiformi.

Tetrao orientalis. Haffelq. it. 278. 12. 43 .

Perdix damafcena. Will. orn. I28.

Francolin. Tournef.it. I. p. 158.t. 158 .

Habitat in Oriente.

Coturnix. I3. T. pedibüs nudis, corpore grifeo-maculato, fuperciliis albis, rectricibus margine lunulaque ferruginea. Fn. fisec. 173 .

Coturnix. Gefin. au. 353. Aldr. orn. l. 13.c. 22. Ionft. au. 69. t.28. Rai. au. 58. Alb. au. I. p.28. t. 30. Tetrao ifraelitarum. Haffelq. it. 279. n. 44 .

Habitat in Europa, Afia, Africa.

Pennae corporis ferrugineae line longitudinali albida. Cbinenes rimum portando manus calefaciunt. Osb. itin. 190. 


\section{PASSERES.}

Roftrum conicum, acuminatum. Nares ouatae, patulae, mudae.

92. COLVMBA. Roftrum rectum, verfus apicem defiendens.

Nares cblongae, membrana molli tumida femitectae.

Lingua integra.

Oenas. I. C. caerulefcens, collo fupra viridi-nitente, fafcia alarum apıceque caudae nigricante. Fn. Jüec. 174.

Oenas f. Vinago. Aldr. orn 1. 15. c. 7. Will. orn. 136. t. 35. Rai. au. 62. Alb. au. 3. p. 39. t. 42. 44. domefti- B. Columba domeftica. Will. orn. 130. t. 32. Rai. au. 59 . ca. Habitat in Europa.

Donneftica parit quais menfe pullos duos, per 9. partus annuos, quod intra 4 annos adfcenderet vltra $\mathbf{1} 8000$; vetita melancholicis; Antequasn cö̈ant prius ofculantwr; intra ingluniem macerata grana in os pulli eutomunt; Mas et femma alternis vicibus incubant; alterque alteram compellit.

gुutturo= 2. C. peEtore inflato.

fa. Columba gutturofa. Will. orn. I2I. t.34. Rai. au.60. Habitat...

cucullata. 3 . C. pennis occipitis reflexo - erectis.

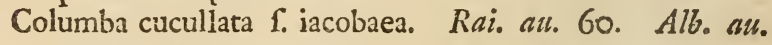
3. p. t. 43 .

Columba anglica 1. ruffica. Gefin. au. 279.

Habitat...

Turbita. 4. C. pennis in pectore recuruatis.

Columba turbita diEta. Rai. au.60. Will. orn. $\mathbf{1 3 2 .}$ Habitat...

tremula. 5. C. cauda erecta multipenni patula.

Columba tremula laticauda. Rai. ๓u. 60.

Hequitat. . 
VI. Paoveres. L 235

92. Colmmba L1735 (Columbuo Rein viso) 

tabellae 6. C. cera lata verrucofa, palpebris nudis.

ria. Columba equeftris. Alb. ail. 2. p.30.t.45.

Columba tabellaria. Rai. au. 60.

Habitat. . .

Longiffime licet deportata feflinanter domum redit, bine olim litteras affixerunt per coelum eunti nuncio.

Vtrun antecedentes omnes Species vere diftinctae fint, docebit aliquando Anatomia.

montana. 7. C. obfcure rufa, fubtus flaua, orbita oculorum, nuda fanguinea.

Columba minor fulua. Edw. alu. II9. t. IIg. Sloan. iam. 2.p. 304. t. 26I. f. I.

Perdix montana. Rai. au. 183.

Habitat in Iamaica.

Macula alba fub oculis, gula et ad axillas.

2fiatica. 8. C. reetricibus cinereis apice albis : intermediis fufcis, orbita oculorum nuda caerulea.

Turtur indicus fufcus. Edw. a1t. $76, t .76$.

Columba fubfufca media, iride crocea, palpebris impinnis cseruleís. Brown. iant. 486.

Habitut in Indiis.

guinea. 9. C. rętricibus cinereis apice nigris, orbita oculorum nuda rubra.

Columba maculis triquetris. Edw. au. 75. t. 75 .

Habitat in Africa.

hifpanica. I0. C. cinerea, remigibus, rectricibus, plumis pedum roftroque albis. It. Wgot. 8.

Habitat. . .

Palum- II. C. ręricibus poftice atris, remigibus primoribus matbus. gine exteriore albidis, cullo vtrinque albo.

Columba collo verinque albo, poftice macula fufca. Fin. Jiuec. 175 .

Palumbus torquatus. Aldr. orn. 1. 15. c. 5. Will. orr. 135.t.15. Rai. all.62. Alb. all. 2. p. 42. t. 46.

Habitat in Europa, Alia.

Cregatimb volant, agris fatis infeftac.

cyanoce- 12. C. capite caeruleo.

phals. Turtur iamaicenfis. Alb. au.aw. 2. p. 45. t. 49 .

Habitat in America. 
leucoce- I3. C. caerulefcens, vertice depreffo albo. phala. Columba capite albo. Catesb. car. I. p. t.25. Sloan. iam2. 2. p. 303. t. 26I.

Columba minor leucocoryphos. Rai. au. 184. Habitat in America Jeptentrionali.

leucopte-I4. C. palpebris nudis caeruleis, iridibus oculorum fuluis.

ra. Turtur indicus fufcus. $E d \mathfrak{w}$. at. $76, t .76$.

Columba fubfufca media, iride crocea, palpebris impinnis caeruleis. Brown. iam. 468.

Habitat in America.

nicobari- 15. C. cauda alba, corpore nigro, remigibus caeruleis, ca. dorfo viridi nitente, pennis colli elongatis.

Columba e nicombar. Alb. au. 3.p. 44. t. $47 \cdot 48$. Habitat in infula Nicombar prope Pegu indicum.

macroura. 16. C. cauda cuneiformi longa, pectore purpurafcente.

Columba macroura. Edw. all. I5. t. I5.

Palumb us migratorius. Catesb. car. I. p.23.t.23.

Habitat in Canada; bybernat in Carolina.

finica. 17. C. reetricibus dorfoque fufcis, abdomine fanguineo, alis flauis, remigibus nigris.

Columba finica. Alb. au. 3. p. 43.t. 46 .

Habitat in China.

indica. I8. C. corpore purpureo, humeris viridibus, fuperciliiis albis.

Columba alis viridibus. Edwo.au. I4. $t$. I4.

Habitat in India orientali.

Pileus caerule fens. Remiges primores nigrae. Reetrices nigrae, fied laterales cinereae. Flexura alarum nigra albo punctata.

hifpida. r9. C plumis minimis erectis per dorfum alasque difperfis.

Habitat in Africa?

Turtur. 20. C. rectricibus apice albis, dorfo grifeo, pectore incarnato, macula laterali colli.

Turtur. Will or\%. I34. t. 35. Rai. au. 61. Alb. au. 2. $p$ 4.3. t. 47 .

Habitat in India. 

93. Alausa 21735 
riforia. 2I. C. fupra lutefcens, lunula ceruicali nigra.

Turtur indicus. Aldr. osn. t. 15. c. 9. Hill. orn. I34. t... Rai. au. 6r. n. 3. Alb. au. 3. p. 42. t. 45 .

Habitat in India, nobis communis Turtur.

pafferina. 22. C. rectricibus remigibusque obfcurioribus, corpore purpurafcente, roftro pedibusque flauis.

Turtur minimus guttutus. Sloan. iam.2. p.305.t. 26 r. f.3. Catesb. car I p. 26.t. 26.

Turtur min!mus, alis maculolis. Rai. att. 184. n. 25 .

Turtur barbadenls minimus. Rai. au. 62. 11.5. Will. orn. $135 \cdot t \cdot 36$.

Columba fy ueftris minima brafilienfis. Rai.au.62.n. 6 . Picuipinima. Marcgr. braf" 204.

Habitat in Amcrics inter tropicos.

Alae extus in rectricibus puntis $\int$. maculis fufcis ad. jperface.

93. ALAVDA. Roftrum cylindrico - fubulatam, rectum, recta protenfüm: Mandibulis aequalibus, bafi deorfum dehifcentibus.

Lingua bifida.

Vnguis pofticus rectior, digito longior.

aruenfis. I. A. rectricibus extimis duabus extrorfum longitudinaliter atbis:- intermediis interiori latere ferrugineis. $F n$. Juec. 190.

Alauda non criffata. Gefin. at.78. Aldr. orn. l. I8. c. 12. Alauda vulgarss. Will. orn. 149. t. 40. Rai. au. 69.

n. I. Alb. au. I. p. 39. t. 4I.

Alauda aruorum. Frifch. au. 3.t. 15. f. I.

Alauda coelipeta. Klein. ass. $7 \mathrm{I}$.

Habitat in Europae apricis.

Volatu perpendiculari in aëre fufpenfa cantillat:

Ecce finun tirile, tirile, funm tirile tractat;

Autunno fole occidinte in wis compellitur.

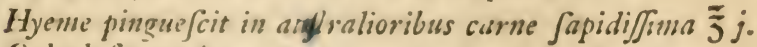
Calculofis noxia.

L $5 \quad$ 2. A.

Columbas ad Pafferes nee ad Gillinas perinere, docet monogamia, ofculatio. incubatus alternus, nutritio pulli, oua pauca, nidificatio, locus in altis. 
pratenfis. 2. A. reftricibus exrimis duabus extrorfum albis, linea fuperciliorum alba. Fn. fuec. I9I.

Alauda pratorum. Aldr. orn. l. 18. c. 15. Will. orn. 150. Rai. au. 6g.n.3. Olin. au. 27.

Alauda fylueftris. Alb. au. I. p. 4.0. t.42.

Habitat in Europae pratis.

arborea. 3. A. capite vitta annulari alba cin̊to.

Alauda reetricibus fufcis: prima oblique dimidiato-alba, fecunda, tertia, quarta macula alba cuneiformi. Fn. Jilec. 192.

Alauda arborea. Will. om. 149. Rai. au. 69. n. 2. Habitat in Europa, gregaria volitans.

campe- 4. A. rectricibus fufcis inferiori medietate, exceptis interftris. mediis duabus albis, gula pectoreque flauefcente. Fn. fuec. 193 .

Alauda minor campeftris. Will. orn. 150. Rai. an. 70. $n .6$.

Habitat in Europa.

triutialis. 5. A. rectricibus fufcis: extima dimidiato alba, fecunda apice cuneiformi alba, linea alarum duplici albida. Habitat in Suecia, in arborunz finnmitate.

criftata. 6. A. rectricibus nigris: extimis duabus margine exteriori albis, capite criftato.

Alauda criftata maior. Rai. au. 69. Will. orn. 15I. t. 40. Olin. au. I3. Alb. au. 3.p. 48.t.52. Aldr. orn. l. Is. c. I8.

Habitat in Europae viis.

Spino- 7. A. rectricibus fufcis: extimis duabus oblique dimidialetta. to-albis. I

Spinoletta florentinis. Rai. au. 70. Will. orn. 152. Habitat in Italia.

alpeftis. 8. A. rectricibus dimidio inferiore albis, gula flaua, fafcia fuboculari pectoralique nigra.

Alauda gutture fiauo. Catesb. car. I. p.32. t. 32.

Habitat in America Septentrionali, et vija a Kleinio Gedani.

Corpus fupra fufcum. Guttur ad pectus vsque flanum. Abdomen album. Peetorale transuerfinm nigrum Fafcic nigra, obtufa, pone finum oris. 

94. Sturmesosigs:

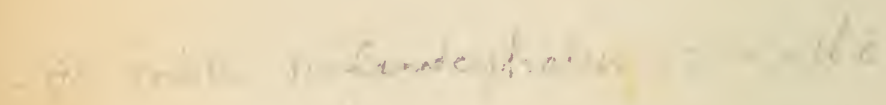


magna. 9. A. fubtus flaua, fafcia pectorali curua nigra: rectricibus tribus lateralibus aibis.

Alauda magna. Catesb. car. I. p. 33. t. 33 .

Habitat in America, Africa.

Corpus fcolopacinum, magnitudine Turdi, totum fubtus flaniffrmum. Pectus macula magna nigricante lunari. Remiges fufcae; fecundaviue teftaceo maculatae. Cauda rotundata: Rectrices 3 laterales maximam partem albae. Vnguis poflicus pedum maior, fed gnagis curuus qnam in roliquis. Roftrum rectum: Maxilla Jupurior teretiufcula, bafi nuda.

94. STVRNVS. Roftrum fubulatum, angulato-depreffuni, obtufiufculum: Mandibula fuperiore integerrima, marginibus patentiufculis.

Nares fupra marginatae.

Lingua emarginata, acuta.

vulgaris. I. S. roftro flauefiente, corpore nigro pun\&tis albis. Fn. fuec. 183 .

Sturnus. Gejn. an. 747. Bell. an. 81. a. Aldr. orn. 1. 16. c. I9. Will. ornith. 144.t.37. Rai. au. 67. n. 1. Alb. au. I. p. 38. t. 40. Olin. an. 18. HajSelqu. itin. 284 .

Habitut in Europa, Africa, victituns infertis. Nidificat in cauis arborum; migrat in Scaniam; garruia imitatrix; Mas witens; gregaria auis.

Luteolus. 2. S. lureus, capite fufco, pectore maculato, alis fufcoluteis.

Sturnus luteus benghalenfis. Edro. cou. 186. t. 186.

Pica maderafparana fturni ad inftar maculata. Rai. ai. 195. n. 7 . t. 1. f. 7 .

Habitat in India.

Contra. 3. S. fufcus, macula oculari fafcia alari abdomineque albis.

Sturnus ex albo et fufco varius benghalenfis. Edw. ats. 187. Alb. at6. 3. p. 20.t. 21.

Habitat in India. 
Cinclus. 4. S. niger, pectore albo.

Motacilla peetore albo, ,corpore nigro. Fn. fuec. 216. Merula aquatica. Gefin. an. 609. Aldr. orn. l. 20. c. 54. Will. orn. 104. t. 24. Rai. an. 66. n. 7. Alb. au. $2 \cdot p \cdot 36 . t .39$.

Habitat in Europa ad aquas: Hyeme ad cataractas, fontesque nos congelandos, vbi defcendit per voragines voriatura Onifos aquaticos Infectaque alia: emergitque non palmipes, byeme folitaria, aeftate plermique gemina verfatur. Mere infectinora nec graniora, naribus laminula fere claufis diftincta a Motacillis.

95. TVRDVS. Roffrum tereti-cultratum, maxilla fuperioreapice deflexo.

Nares nudae, fuperne membranula femitectae.

Lingua lacero-emarginata.

vifciǔo- I. T. dorfo fufco, collo maculis albis, roftro flauefcente. rus. Turdus vifciuorus maior. Rai. au. 64. "n. I. Will. orn. 137. Aldr. orn. l. I6. c. I.

Habitat in Europa.

Sibinuet ipfi malum cacat diffeminator Vifci, Ilicisque.

pilaris. 2.T. rętricibus nigris: extimis margine interiore apice albicantibus, capite vropygioque cano. Fn. fuec. I88. Turdus pilaris. Gefn. au. 753 . Aldr. orn. l. 16. c. 3. Will. orn. 138. t. 37. Rai. au. 64. n. 3. Alb. au. I. p. 34. t. 36 . Frifch. au...t.26. f. 1. 2. Olin. ail. 25 .

Habitat in Europa.

Romani in ornitbone maxima diligentia alebant Twrdos, altiles reddebant, care pendebant, tefte Varrone, Plutarcho.

Nil melius Turdo, nil vulua pulcbrius ampla. Horat.

iliacus. 3. T. alis fubtus flauefcentibus, reetricibus tribus lateralibus apice vtrinque albis.

Turdus iliacus. Rai. au. 64.n.4. Klein. au.66. Aldr. orn. I. 16. c. 4 .

Turdus vifciuorus maurus. Alb. all. I. p. 3I. t. 33 .

Habitat in Europa. 
95 turono Lis3s 

Linea nulla fuperciliovam alba. Caro ob amaritiem mimes accepta.

muficus. 4. T. alis fubtus ferrugineis, linea fuperciliorum albicante. Fn. fuec. 189 .

Turdus fimpliciter diftus vifciuorus minor. Will. orn. 138. t. 37. Rai. an. 64. n.2.

Turdus muficus. Frifçb. all. t. 27. $f$. I. 2. Alb. al. I. p. $32,33 . t .35,34$.

Habitat in Europae Jyluis; nido ex luto.

E funmitate arboris vere Lufciniam cantilena innitatur; Diffeminator cum praecedentibus Inniperi.

Canorus. 5. T. grifeus, fubtus ferrugineus, linea alba ad latera capitis, cauda rotundata. Cbin. Lagerftr. Ir.

Turdus chinenfis. Osb. it. 309 .

Turdus fufcus benghalenfis non maculatus. $E d w$. aw. 184. t. 184 .

Baniah boue benghala. Alb. au. 3. p. 18. t. I9.

Habitat in Benghala, China.

rufus. 6. T. ferrugineus fubtus dilutior maculatus, remigibus vnicoloribus, cauda rotundata.

Turdus ruffus. Catesb. car. 1. p. 28. t.28.

Habitat in America Jeptentrionali et meridionali.

polyglot- 7 . T. obfcure cinereus, fubtus pallide cinereus, macula tos. alarum albida.

Turdus minor caeruleo-albus immaculatus. Sloan.iam. 2. p. 306.t. 256.f. 3. Catesb. car. I. p. 27. t. 27 . Rai. all. 185. n. 3I.

Mocking - bird. Kalm. itin. 2. p. 335.

Habitat in Virginia.

Eximia voce cantillat et cantu inftruitur.

Orpheus. 8. T. dorfo fufco, pectore ręricibusque lateralibus albidis, alis fafcia alba. Bromn. iam. 469 .

Turdus cinereus minor. Edw. all. 78.t.78.

Habitat in America.

Cauda longa rutusdata. Reefrices extimae albue. $R$ terva elenatus cantilena Jpectatorem rapit in fui admirationem.

plumbeus.

9. T. caerulefcens, gula atra.

Turdus vifciuorus plumbeus. Catesb. car. I.p.30.t.30. Habitat in America. 
crinitus. I0. T. capite colloque caerulefcente, abdomine flauefcente, dorfo virefcente, rectricibus remigibusque rufis, $\mathrm{ca}$ pite criftato.

Mufcicapa criftata, ventre luteo. Catesb. car. I. p. 52 . t. 52 .

Habitat in America.

rofeus. Ir. T. fubincarnatus, capire, alis caudaque nigris, occipite criftato.

Merula rofea. Aldr. omn. l. 16. c. I5. Will. orn. 143 . Rai. all. 67. Edw. all. 20.t. 20.

Turdus rofeus, capite ex niggo, caeruleo et cirtho setro compto, alis et cauda nigris. Klein. au. $7 \mathrm{I}$.

Habitat in Lapponia, Helueria.

Merula. 12. T. ater, roftro palpebrisque fuluis. Fn. Juec. I84.

Merula. Bell. au. 30.b. Gejn. au. 603. Aldr. orn. i. 16. c. 6. Will. orn. 140. t. 37. Rai. au. 65.n. x. Alb. au. x. 35.t. 37. Frifch. au. 27. f. 1. 2. Olin. ale. 29.

Fabitat in Europae fylius, Inniperi diffeminator.

Fenina fufica eft. Pullus prinzo anno, etiam mafculus, roftro nigro.

torqua- I3. T. nigricans, torque albo, roftro flauefcente. Faun. tus. fiec. 185 .

Merula torquata. Gefn. an. 607. Aldr. orn. l. 16. c. II. Will. orn. 143. t. 37. Rai. au. 65. n." 2. Alb. au. I. p. 37.t. 39. Frifch. au. 30.f. I. 2.

Habitut in Europa.

folitarius. I4. T. caeruleus, remigibus rectricibusque nigris, abdomine lineolis cinereis vndulato.

Paffer folitarius. Willugh. orn. I9I. Rai. au. 66. Edwo. au. 18. t. 18. Olin. au. 14. Act. VpS. 1750. p. 21. Habitai in Oriente.

Mas caeruleus; Femina cinerea, fubtus lineolis albis et cinereis vndulata.

arundina- I5. T. grifeus, fubtus albidus lineolis transuerfis lunatis ceus. fulcis, pedibus caerulefcentibus. $f$

Turdus mulicus paluftris dumetorum arundinum. Klein. au. $179 . f .3$.

Habitut in Arundinetis Europae Septentrionalis. 

96. Loxia. Lily35.

96a. Coccothraustes Klein nso. 
Mes indefinenter cantillans, toto dic, fub incubatu $\mathrm{Fe}$ minae in terra nidnlantis. Klein.

virens. 16. T. nigro-virens, fubtus iutefcens, fuperciliis albis.

Oenanthe americana, pectore luteo. Catesb. car. I. p. 50. t. 50 .

Habitut in America.

96. L OXIA. Roftrum conico-gibbum, fronte fubcaluum: Mandibula -inferior margine laterali inflexa. Nares in bafi roftri.

- Lingua integra.

Curüro- I. L. roftro forficato. Fn. fuec. 177 .

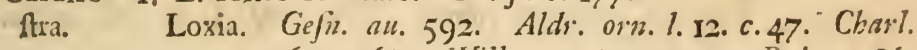
onom.68. t.6g. Will. orr.181.t.44. Rai.au. 86. Alb. ornith. I. p. j8. t. 6I. Fiifch. au. 2. t. II. f. $3 \cdot 4$ :

Habitat inter Europse Abietes, frrobilos enucleans, at non in ipfa arbord, difeminator Abiețis roftro fingulari.

Cocco- 2. L. linea alarum fimplici alba, rectricibus latere tenuiore thrauftes. bafeos albis.

Coccothrauftes. Gefin. all. 276 . Aldr. oinith. l. I6.c. 20. Ionft. a\%...t. 37 . Cbarlet. ononi. 84. t. 85. Olin. ait. 37. Rai. au. 85. Alb. orn. I. p. 54. t. 56. Edr. all. IS8. t. ISS. It. fian. 332. n. I. * Frifch. all. I. t. 4. f: 2. 3 .

Habitat in Europa cuefraliori, Cerafa cnucleans, diffeminans.

Enuclea- 3. L. linea alarum duplici alba, rectricibus totis nigricantor. cantibus. Fr. fulc. 176 .

Rubicilla maxima canadenfis. Edwr. att. 123. t. 123. 124.

Habitat in Sueciae fummae, Canadae Pinctis, vicritans frobilis; diffeminator Pini. Nocfu egregie canorus; migrat byeme ad auflrales Suecinc prouincias, I If faliam transit fine ostobris. Itunior ruber, Senior flawus.

Pyrthuia. 4. L. artubus nigris, rectricibus caudae remigumque pofticarum albis. Fin. Jüe, 178. 
Rubicilla f. Pyrrhula. Gefn. an. 733. Aldr. orn. l. I7. c. 31. Ionjt. au. . t. 43. Will. ornith. 130. t. 43 . Rai. au. 86. Alb. orn. I. t. 59. 60. Frijch. au... t. 2.f. 1. 2. Olin. au. 40.

Habitat in Europae fyluis, Sorbi diffeminator. Mas jibtus ruber, Femina Jubtus fiufco-cinerea.

Cardina- 5. L. rubra, facie nigra. Chin. Lagerftr. 13.

lis.

Cardinalis. Will. orn. 245. t. 44. f. 8 .

Coccothrauftes capenlis ruber, roftro hiante. Pet. gaz. 16. t. I6. $f .7$.

Coccothrauftes ruber. Catesb. car. I. p. $38 . t .38$. Frijchb. ormuth. r. t. 4. $f$. $x$.

Coccuthrauftes indica criftata. Alb. orn. I. p. 55. $t$. 57. et 3. t. 6I. Aldr. ornith. l. 16. c. 2I. Rai. all. 85 .

Pfittacus criftatus ruber. Seb. muf. I. p. 96. t.60. f. 4 .

Habitat in America Septentrionali.

Domini- 6. L. nigra, capire gulaque coccineis, pectore, abdomine cana. remigumque margine albis. Cbin. Lagerftr. I4. Rubicilla americana. Will. orn. I80. Rai. au. 86. Cardinalis dominicanus. Edvo. au. $127 . t$. 127 . Guiraliuica. Marcgr. braj:211. Habitat in Brafilia.

criftata. 7. L. albicans, fronte criftata, vropygio pedibusque rubris, reetricibus intermedis longiffimis.

Habitat in Aethiopia. I. Burmannus.

Anis inter maximas jii generis. Crifta Maris rubra, Feminae alba. Pectus in mare rubrum. Rectrices cinereae: intermediae duae duplo longiores.

mexica- 8. L. rubra, alis nigris.

- na. Auis mexicana rubra pafferis fpecies. Seb. muf. I. $p$. IOI. $t$. $95 \cdot f \cdot \mathrm{I}$.

Habitat in America auftrali.

eryoce- 9. L. cinerafcens, capite purpurafcente, pectore albo maphala. culato.

Paffer paradifaeus, capite rubro, pectore nigro albo maculato. Edw. alw. 180. t. 180.

Habitat in Africa. 


Corpus fupra cinereum, grifeo maculatum; fubtus totum fufcum maculis albis rotundis. Alae fufcar. Remiges margine grifeae. Cauda nigra, forficata. Caput purpuseums.

flaúicans, IO. L. flaua, dorfo virefcente, capite fuluo. Cbin. Lagerftr. 17.

Habitat in Afia.

oryziứo. II. L. fufca, temporibus albis, roftro rubro. Cbin. La. ra. gerftr. 116.

Loxia remigibus reetricibusque nigris. $M u \int . A d$. $F r$. I p. I8.

Fringilla capite et gula nigta, temporibus albis. Osb. iter. 103 .

Oryziuora. Edw. aut 4I. t. 4I. 42.

Paffer indicus. Aldr. orn. l. 15.c.24.

Habitat in Afia et Aethiopia inter Orytam.

panici- 12. L. nigra, ala fpuria alba, roftro incarnato.

vora. Fringilla nigra. Alb. aul.3.p. 65.t.69.

Habitat in India.

An praecedentis Femina?

punctu- 13. L. ferruginea, abdomine nigto albo-maculato.

lata. Paffer indicus fufcus, ventre ex albo et nigro punctato. Edw. alu. 40.t. 40.

Habitat in Afia.

hordea- 14. L. fulua, temporibus albis, cauda pectoreque atris. cea. Muf. Ad. Fr. 2. p. .

Habitat in Indiis.

Corpus magnitudine Motacillae albae. Grifea funt $\mathrm{Hu}$ meri, Femora, Ani regio, margines Rectricum. Nigra funt Tempora ad roftrum vsque, Alae ct Remiges, Cauda et Rectrices, Pectus. Fulua fint Caput, Collum, Vropygium.

finguini- 15 . L. grifea, fubrus alba, roftro pedibusque fanguineis. roftris. Clsin. Lagurftr. 15.

Habitat in Afia.

Aftrild. 16. L. grifea fufco-undulata, roftro temporibus pectorereque cocsineis.

Waxbill. Edw. aแ. 179, t. 179 . 
Habitat in Canariis, America, Africa.

Magnitudo Reguli; Jipra griféa fulco undulata; fubtus pectore purpuren. Cauda rotundata, griféa lineolis transucrjis fufcis numerojis. Roftrum coccineum, gibbum. Faficia coccinea per oculos ducta.

cyanea. 17. L. caerulea, remigibus rectricibusque nigris. Cbinenf. Lagerftr. I8.

Coccothrauftes caeruleus. Edw. au. I25. t.125.

Habitat in Angola.

Lineola. 18. L. nigra, linea frontali temporibusque albis.

Habitat in Afia.

Corpus magnitudine Pari; fupra nigrum, e cacruleo nitidum; fubtus album. Roltrum nignum, gibbum. Lineca a fironte ad verticeni duct a alba. Macula alba Jipra maxilla inferiore. Remiges nigrae: Primores baji anteriore aibae, unde macula alarum alba. Cauda nigra, bifurcaia.

mexica-, 19. L. grifea, fronte gula vropygio fuperciliisque luteis. na. Linaria mexicana, capite flauo. Edw. au. 44. t. 44.

Habitat in America. MHe. DeGeer.

Magnitudo Fringillae. Fajciar duae cinerafcentesque in tectricibus alarum. Cauda integra.

Chloris. 20. L. flauicanti-virens, remigibus primoribus antice luteis, ręricibus lateralibus quatuor bafi luteis.

Fringilla eadem. Fr. fuec. 202.

Chloris. Gefir. au. 259. Will. orn. I29. Rai. au. 85. n. 4. Alb. au. I. p. 56. t. 58. Frijch. au. l. 8. f. 3. 4. Aldr. orn. l. I8.c. Is.

Habitat in Europa.

Media quafi inter Loxias et Emberizas, fed rofrumb craffius quam in Emberizis.

butyra- $2 \mathrm{r}$. L. virefcens, fronte fuperciliis pe?tore abdomineque cea. fluuis, caudae apice albo.

Habitat in India.

Corpus magnitudine et figura Ligurini, viride, fufco maculatum; fubtus totum flaum. Frons ct inde linea fupra oculos flauce. Tempora flaus. Cauda nigriGans, bifiuca, apice alba. 


Collaria. 22. L. flauefcens, pectore collarique flauis, temporibus nigris.

Habitat in Indiis.

Roftrum nigrum. Pedes fufci. Frons calua. Genae nigrae. Pectus et ferme Collare flana. Corpus viride flauefcens, magnitudine Pari minimi.

bengha- 23. L. grifea, pileo flauo, temporibus albidis, abdomine lenfis. albido fufco-maculato.

Paffer benghalenfis, capite flauo. Edw. au. 189. t. I89. Alb. ali. 2. p. $48 \cdot t \cdot 52$.

Habitat in Benghala.

malaba- 24. L. cinerea, remigibus rectricibusque nigris, gula ano. rica. que albis.

Habitat in Indiis.

Corpus magnituline et facie Pari. Roftrum nigrum. Gula alba. Remiges et Rectrices nigrae. Ani regio albida.

furca. 25. L. fufca, fubtus albida, remigibus a tertia ad nonam bafi omnino albis. Cbin. Lagerftr. Ig.

Habitat in Benghala.

melano- 26. L. lutea, capite atro.

cephala. Coccorhrauftes gambogia. Alb. alu. $3 \cdot p \cdot 58 \cdot t .6 \approx$

Habitat in Guinea.

eana. 27. L. cana, remigibus ręricibusque fufcis, pedibus rubris.

Linaria cinerea orientalis. Edwo. au. 179. t. 179. $f$. $\mathrm{r}$.

Habitat in Afia.

Margines rectrican et oris regio albida.

nigra, 28. L. nigra, macula alba humeri remigumque duarum exteriorum.

Rubicilla minor nigra. Catesb. car. p. 68. t. 68. Alb. als. 3.p. 65.t.69.

Habitat in America auflrali.

caerulea. 29. L. cacrulea, alis fufcis fafcia bafeos purpurea.

Coccothrauttes caerulea. Catesb. car. I. P. 39.t. 39 .

Habitat in America. 
violacea. 30 . L. violacea, fuperciliis gula anoque rubris.

Coccothraufter purpurea. Catesb. car. I. p. 40. t. 40. Habitat in America.

minuta. 3I. L. grifea, vropygio fubtusque ferruginea, remigibus 4, 5, 6, bafi vtrinque albis, cauda integra. Rolander. Habitat Surinami.

Anicula vix Regulo maior; Roftrum breue, craffum, jubobtufim.

bicolor. 32. L. fufca, fubtus rubra.

Rubicilla fufca minima. Edw. at. 83.t. 83. f. x.

Habitat in India.

Magnitudo L. minutae.

97. EMBERIZA. Rofrum conicum.

Mandibulae bafi deorfum a fe invicem difcedentes: inferiore lateribus inflexo-coarctata; fuperiore anguftiore.

niưalis, I. E. remigibus albis: primoribus extrorfum nigris, rectricibus nigris : lateralibus tribus albis.

Alauda remigibus albis: primoribus extrorfum nigris, reEtricibus nigris: lateralibus tribus albis. Act. Stockho 1740. p. 368. t. 1. f. 9. Fn. füec. 194. t. 1. f. 194. Pied-Chaffinch. Alb. all. 2. p. 50. t. 54. et 3. p. 67. t. $7 \mathrm{I}$.

Auis ignota piperini. Gepro au. 798 .

Fringilla albicans. Aldr. orn. l. 8. c. 357 . t. 15.f. I. Montifringilla calcaribus alaudae. Will. orn. IS7. t. 77 . Rai. aus. 88 .

Miliaria niuis. Frifcb. alt. 2. t. 6.f. 1. 2.

Auis niuis. Martenf. Spitsb. 53.t.K.f.B. Edw. au. $\mathrm{I} 26 . t$. 126 .

Habitat in alpibus Lapponiae, Spitsbergae, ad finum Hudfonis alibique, terrae infidens; inten/iure byeme ad nos migrans; auena nutritur, Sapidifima; bieme alba; noctu vigil.

Calan- 2. E. grifea nigro-maculata. Fn. fuec. 206. It. fcan. dra. 292. t. 4 . 
97. Inberiza Lifro. (Ambriza Innorys) 

Emberiza alba. Giefn. au. 564. Rai. all. 93. Will.orn. 195. t. 40. Alb. all. 2. p. 46.t. 50. Olin. all. 50. Alaudae congener. Aldr. orn. .

Miliaria cana. Frifcb. atu... t.6. $f .4$.

Habitat in Europa.

Praecedinti valde affinis.

Hortuld- 3. E. remigibus nigris: primis tribus margine albidis, tena. Atricibus nigris: lateralibus duabus extrorfum nigris. Fn. filec. 208.

Hortulanus. Giefin. all. 569. Ionft. all...t. 37. Will. orn. 167. t. 40. Rai. au. 94. n. 6. Olin. au. 22. Albin. au. 2. t. 50.

Miliaria pinguefcens. Frifch. au. 2. t. 5.f.3.4.

Habitat in Europa.

Oculorum orbita f. palpebrae midac flauae, vt oculi ansmulo flauo cingantur nudo, et caput fibtus ad maxillam inferioren flaum.

Citrinel- 4. E. reftricibus nigricantibus: extimis duabus latere intela. riore macula alba acuta. Fin. fuec. 205.

Emberiza flaua. Gefn. atl. 653. Will. onn. 196. t. 40. Rai. au. 93.n.2. Alb. au. I. p.63.t.66.

Luteae alterum genus. Aldr. orn.

Miliaria lutea. Frifch. au. 2. t. 5. f.2.

Habitat in Europa, nidificat bumi in pratis.

Hyeme domeftica; acftate colligit laruas Brafficae.

Orix. 5. E. grifea, fronte abdomineque nigris, collo vropygioque fuluis, roftro nigro.

Paffer angolenfis, capite nigro, collo et vropygio flauis. Edw. au. 178.t. 178 .

Habitat in Africa interiore.

Quelea. 6. E. dorfo grifeo, fronte nigra, roftro coccineo.

Habitat in India.

Roftum coccincum, craffum Loxiae, at non incurutum, maxillis Limberizac. Frons z'ndique circun roflewn atra. Capur, Humeri, Alae nigro grifeoque maculatae vt Pafjeris domeflici. Peetus it Abdomen albo jubincarnata. Remiges Redricisque nigricantes margine z:ndique cinerco. Pedes V'nguesque incarnatio 
militaris. 7. E. fufca, pectore gula humerisque fanguineis.

Turdus ater, pectore coccineo. Muf. Ad. Fr. I. p. Is.

Chin. Lagerftr. I2.

Rubicilla fufca maior. Edw. au. 82.t. 82.

Habitat in America, Afia.

Magnitudo Sturni. Color fupra e fcolopacino fufcus, fiubtus fanguineus. Roftrum bafi fubcaluum. Remiges fuffae: I, 2 margine exteriore grifeae. Rectrices aequales, fufcae: apice fajciis pallidioribus variae.

3teata. \&. E. atra nitens.

\section{Habitat in India.}

Tota, ctiann roftro, atcrinta, corpore fupra parum $c$ caevuleo virefcente, nitido vt in Coruo; magnitudo Turdi.

familia- 9. E. grifeo-maculata, apicibus rectricum albis, dorfo poris. ftico flacio.

Motacilla capite et roftro nigro, vropygio luteo. Osb. iter. 102.

Habitat in Afia.

Corpus magnitudine Ligurini, fupra cinereum maculis fufcis, fubtus cinereum. Dorfum fub alis et verfus caudam flaum. Tectrices caudae albae.

flitueola. 10. E. grifea, facie flaua.

Habitut in calidis regionibus.

Corpus magnitudine Ligurini. Frons et Gula flauae.

plittacea. Ir. E. cinereo-fufca, alis fuluis, rectricibus duabus longiffimis.

Fringilla brafilienfis. Seb. mus. I. p. 103. t. 66. f. 5 . Habitat in America auftrali.

paradi- i2. E. fufca, pectore rubro, reetricibus duabus longioribus raea. acuminatis, intermediis duabus longiffimis.

Paffer indicus. Aldr. orn. l. 15. c.23. Will. orm. I84. t. 45 .

Paffer pfittacus indicus, cauda longa.' Pet. gaz...t. 55. $f$. I.

Paffer pectore rubro, cauda longiffima. Edw. au. 86. $t$. 86.

Habitat in Africa.

Nigra eft fed ceruice et pectore coccineo. Deponit quotannis longas caudae pennas, vti Pauo. 
Gen. Tanagra. 2. 1964. ibo. th. Fy. St. 2. . . 30.

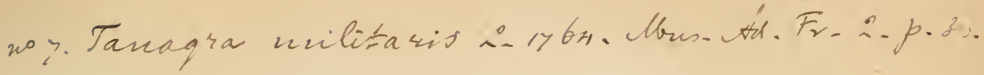

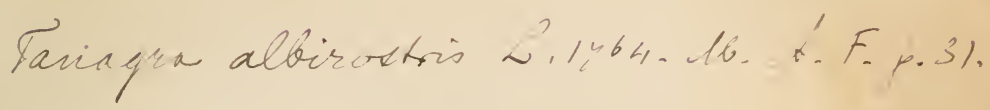


$90 \frac{\text { Pringilla L1y35. (Passer Klein 1950) }}{\text { (Linania Klim 1y50) }}$ 
Ciris. I3. E. capite caeruleo, abdomine fuluo,' dorfo viridi, pennis viridi-fufcis. Act. Stockb. 1750 . p. 278. t.7. f. I.

Fringilia tricolor. Catesb. car. I. p. 44. t. 44.

Fringilla tricolos mexicenlis. Ed w. all. 130. t. I30.

Fringilla chinenfis. Alb. aul. 3. p. 64.t.68.

B. Linaria caerulea. Catesb. car. 1. p. 44.t. 44 .

Habitat in America.

Caput tott:met Collum caeruleumn. Dorfum, Alae Caudague fitpra viridia; tota fibtus lutea, fed l'eetus antice fulutm. B. Femina caevulea folo abdomine albo, bieme fere griféa eltadit.

Alario. I4. E. capite pectoreque atro, abdomine albo, alis rubris. Paffer capenlis. Alb. all. 3.p. 63.t.67.

Habitat ad Cap. b. Spei.

98. FRINGILLA. Rofrum conicum, rectum, acutum.

oryziưo- I. F. fufca, ceruice fulua, macula alarum dotfoque poftico ra. albis.

Hortulanus carolinenfis. Catesb. car. I. p. 14. t. 14.

Emberiza carolinenlis. Klein. au. 92.

Habitat in Cuba, vbi autunno confiunt a Oryza, feninae folae defcendunt in Carolinam.

Femina grifea tota a mare colore differt.

coelebs. 2. F. attubus nigris, remigibus vtrinque albis: tribus primis immaculatis, rectricibus duabus oblique albis. Fr. fiuec. $199.1 .2 . f .199$.

Fringilla. Gefir. au.387. Aldr. orm. 1. 18. c.6. Will. orn rg6.t. 45.f. 4. Rai. au. 8S. Alb. au. I. p. 60. t. 63. Frifcb.au...t. I.f. I. 2. Olin.au. 3I. Habitat in Europa.

Femina fola migrat per Belgium in Italiam. Mas capite pileato, vere mutato jouto acftaten annunciat.

Monti- 3. F. alarum bafi fubtus flauifima. In fucc. 199. t.2. $f$. 19S. fringilla. Montifringilla f. Fringilla montana. Ionft. au. 99. $t$. 38. Will. orm. 187. t. 45. f. 5. Rai. a11. SS. Alb. as. 3. p. 60.t. 64. Fivijs. atu. .t. 3.f. 2.3. Olin. all. 32. Alli: orn. l. I8. c. 7 .

Habitat in Europa. 
lulenfis, 4. F. fufea, pectore humerisque rufis, alis nigtis macula rufa. Fn. liuec. 197.

Habitat in Suecia.

lapponi- $5 \Gamma_{0}$ capite nigricante maculato: macula pone oculos alc. ba. Fu. filec. 196.

Fringilla maior. Alb. au. 3.p. 59, t.63.

Habitat in Lapponia.

Syluatica. 6. F. artubus remigibus reetricibusque nigris: duabus vtrinque extimis a medio extrorfum albis, Fn. Juec. 200.

Habitat in Suecia.

melan- 7. F. grifea nigro-punctara, area a roftro per latera colli cholica. atra.

Einberiza fufca americana. Edw. au. 85.t. 85 .

Habitat in America auftruli.

erythroph-\$. F. nigra rubro relucens, abdomine rufefcente, macula thalma. alarum alba.

Paffer niger, oculis rubris. Catesb. car. I. p.34.t. 34 . Habitat in America.

Cardue- 9. F. remigibus antrorfum luteis : extima immaculata, relis. Aricibus duabus extimis medio reliquisque apice albis. Fn. Guec. 195 .

Carduelis. Giefn. au. 242. Aldr. osm. l. 18. c. 3. Ionft. au. 97. t. 37. Will, orn. IS9. t. 46. Rai. au. 89. 11. I. Merian. inj.t. 173. Alb. aw. I. p. 61. t. 64. Frifch. au. . t. I. f. 3. 4. Olin. au. Iо.

Habitat in Europae iuniperetis; byeme in capitatis plantis.

Melba. Io. F. facie caudaque rubris, abdomine albo nigroque vrdato, dorfo viridi.

Carducli affinis viridis. Edwo. au. I28. t. I28.

Habitat in China.

Amanda- II. F. reetricibus purpureis medietate poftica atris. va. Amandava. Alb. au. 3.p. $72, t \cdot 77$.

Habitat in India oricntali.

Mas totus purpurcus; Femina cinerea exceptis roftro et re?tricibus. 


Gyrola. I2. F. viridis, capite rubro, collari flauo, pectore caeruleo. Paffer viridis, capite rubro. Edwo. all.23. t. 23 . Habitat in America.

rubra. I3. F, rubra tota.

Mufcicapa rubra. Catesb. car. I. p.56.t. 56. Habitat in America.

triftis. I4. F. flaua, fronte nigra, alis fufcis.

Carduelis americana. Catesb. cas. I. p. 43. t. 43.

Habitat in America feptentrionali.

Zena. 15. F. capite nigro, fafcia alba alarum fupra infraque oculos, pectore fuluo.

Fringilla bahamenfis. Catesb. car. I. p. $42 . t .42$.

Habitat in America meridionali.

brafilia- 16. F. cauda cuneiformi, corpore rufefcente, temporibus

na. vropygio abdomineque violaceis, roftro rubro.

Fringilla coloribus rufo et caeruleo brafilienfis. $E d w$. au. I9I. t. I9I.

Habitat in Brafilia.

butyra- 17. E. virens, fuperciliis pętore abdomineque flauis, recea. migibus primoribus margine exteriore albis.

Chloris indicus. Edw. all. 84.

Habitat in Madera.

Similis Loxiae butyraceae, fid roftrum mimus.

Canaria. I8. F. roftro corporeque albicante, rętricibus remigibusque virefcentibus. Fin. fitec. 207.

Canaria. Giefin. all. 240. Aldr. orn. l. IS. c. 5.

Paffer canarienfis, lonfl. all. t. 36. Will. orn. 192.t. 46. Rai. au. 91. n. 6. Alb. au. I. p. 62. t. 65. Frifch. au. 2. t. 12. f. 5. Olin.au. 7 .

Habitat in Canariis infiulis.

Seminilus Ploalaridis imprimis victitans; bybrida parit, fed non eius nepos.

Spinus. Ig. F. remigibus medio luteis: primis quatuor immaculatis, rectricibus bafi flauis apice nigris. Fn. finec. 203 . Acanthus auicula. Gijir. all. I.

Spinus f: Ligurinus. Aldr. orn. I. I8. c. 4. Will. orn. 192. t. 46. Rai. all. 91. n. I. All, au. 3. p.71.t. 76. Frifch, all. 2. t. 11. f. 1. 2. Olin. all.15.

Habitat in Europae imniperitis. 
flammea. 20. F. fufca, crifta flammea. Faun. fuec. 20r.

Linaria f. Luteola nigra. Klein. au. 93.

Haủtat in Europa.

flaüiro- 2I. F. fufca, roftro flauicante. Faun. Juec. 204. ftris. Habitat in Europa.

cannabi- 22. F. remigibus primoribus re?ricibusque nigris vtroque na. margine albis. Fn. fitec. 209.

Linaria rubra maior. Will. orn. I9I. t. 46. Rai. au. 90.n.2. Alb. au. 3. p. 68. t.72. 73. Frifch. au. 2. $t .9 . f .1 .2$.

Habitat in Europa.

Linaria. 23. F. remigibus rectricibusque fufcis margine obfolete pall:do, litura alarum albida. Fn. Juec. 2 IO.

Linaria rubra. Gefin. au. 591. Aldr. 01k. l. I8. c. 9. Will. orn. 19I. Rai. au. 9I.n.3. All. aw. 3.p.70. t. 75. Frifch. all. t. 10. $f \cdot 3 \cdot 4$.

Habitat in Europa.

angolen-24. F, dorfo fufco, abdomine caudaque caeruleis.

fis. Fringilla ventre caeruleo. $E d w$. au. I3I. t. I3I.

Habitat in Angola et Africa.

violacea. 25 . F. violacea, fronte fubtusque flauiffima. Muf. Ad. Fr, 2. $p$...

Habitat in Calidis reginnibus.

Nigro-caerulea funt Dorfum, Collum, Remiges, Rectrices jiipra. Flauiffma fint Frons, Pectus, Abdo= men, Collum fibtus. Roftrum breuifimum, trigonum, nigrum apice incuruo.

Schoeni- 26. F. rectricibus fufcis: extimis duabus macula alba cuclus. neiformi, corpore grifeo nigroque, capite nigro.

Fringilla capite nigro, maxillis rufis, torque albo, corpore rufo-nigricante. Fn. fuec. 2 II.

Paffer aquaticus 1 . Schoeniclos. Ge $n$. au. 652 .

Iunco f. Paffer arundinaceus. Aldr. orrz. 529 .

Paffer torquatus in arundinetis nidificans. Will. orm. I96. Rai. au. 93. n. 3 .

Cannevarola. Alb. att. 2. p.47.t. $5 \mathrm{I}$.

Habitat in Europa.

Similis $F$. domefticae gula nigra, fed capite nigro; collari albo, canda maxime forcipata. 


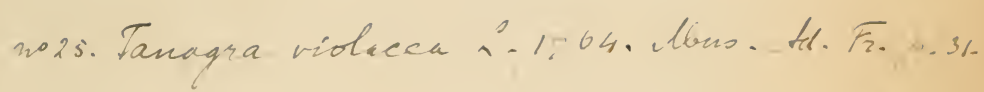



domefti- 27. F. remigibus reetricibusque fufcis, corpore grifeo ni-

ca. groque, fafcia alarum alba folitaria.

Fringilla remigibus rectricibusque fufcis, gula nigra, temporibus ferrugineis. Fn. Jüec. 212.

Paffer domefticus. Gicfin: au. 643. Aldr. om.l. I5. c. I0. Will. orn. 182. t. 44. Rui. au. 86. Alb. au. I. p. 5. 9. t. 62. Frijch. alt... t. \&. f. 1. 2. Olin. au. 42 .

Habitat in Europa prope rura.

Salacifinnus qui vigelies faepe coit. Hortis peffima.

montana. 28. F. remigibus reßricibusque fufcis, corpore grifeo nigroque, alarum fafcia alba gemina.

Pafler montanus. Aldr. ornith. t.15. c. 15. Olin. au. 46. Rai. aut. 87.

Habitat in Europa.

Simillima Fr. donefticae fed minor, et fafcia duplex in alarum tegetibus alba $\int$ tectrices alaruni primi fecundique ordinis albi, at in $F$. domeffica toutum jêcundi.

chinen- 29. F. ferruginea, capite atro, roftro caeruleo.

fis. Paffer chinentis. Alb. all.2. p.49. t.53.f. I. Edw. au. 43 . t. 43 .

Habitat in China.

Roftrun craffun fere Losiac.

hyemalis. 30 . F. nigra, ventre albo.

Pafter niualis. Catesb.car. I. p. $36 . t .36$.

Habitat in America.

Zena. 31. F. capite pectoreque nigris, dorfo alis caudaque obfcure virefcentibus.

Pafler bicolor bahamenfis. Catesb. car. I. p. 37. t. 37.

Habitat in America. 
194 AVES PASSERES. Motacilla.

99. MOTACILLA. Rnftrum fubulatum, rectum: Mandibulis fubaequalibus. Naves obouatae. . Lingua lacero-emarginata.

Lufcinia, I. M. rufo-cinerea, genuum annulis cinereis. Fn. fuec. 221.

Lufcinia. Gefn. au. 592. Aldr. orn. 1. 18. c. 2. Will. orn. 16I. t. 4I. Rai. au. 78. n. 2. Alb. au. 3. p. 49. t. 53 .

Habitat in Europae frondo fis, noctu vefpereque cantillans: tanta vox, tam paruo in corpufculo, tam pertinax $\int p$ iritus; jpiritu prius deficiens quan cantu. Plin. X: 29. Migratrix auis, nutritur ouis Formicarun!.

Calidris. 2. M. fupra fufco-virefcens, fubtus fulua, linea oculari fubocularique nigra.

Lufcinia americana. Edwo. au. I2I. t. I2I. $f .2$.

IEterus minor nidum fufpendens. Sloan. iam. 2. p. 299. Rai. au. 184. $n .27$.

Habitat in America.

Rectrices apice luteac.

modula- 3. M. fupra grifeo-fufca, tętricibus alarum apice albis, ris, petore caerulefcente-cinereo. Fin. Juec. 223. Curruca cantu lufciniae. Frijch. au. t. 21. $f \cdot 3 \cdot$ Klcin. ait. 73 . $t$. I.

Habitat in Europa.

Schoono- 4. M. teftaceo-fufca, fubtus pallide teftacea, capite macubaenus. lato. Fin. Jisec. 222.

Habitat in Europa.

campe- 5. M. grifea, capite cinereo, rectricibus concoloribus, ftris. abdomine albido.

Curruca iamaicenfis. Ediv. all. 122. t. 122. f. 2.

Habitat in Iamaica.

Curruca. 6. M. fupra fufca, fubtus albida, rectricibus fufcis: extima margine tenuiore alba. Fn. Jisec. 233.

Cur-

Motacillae plerneque infectinorae, nic graninorae, binc byemens verfus e borealibus ad meridionales terras migrant. 
79. Lotacilla Lis3s.

99a. Luvainia Lirss (Ficedula Klein 1750)

(rvochlodyteo Klein nso.) (Sylvia Klein vyso) 

Curruca. Gefri. alt. 370. Aldr. orn. l. 17.c.34. Will. orn. 157. Rai. au. 79. n.6.

Habitat in Europa.

Hippo- 7. M. virefcente-cinerea, fubtus flauefcens, abdomine lais. albido, artubus fufcis. Fn. fuec. 234 .

Ficedula feptima. Aldr. orn. l.17. c. 37. Rai. aw. $79 \cdot n .7$.

Habitat in Europa.

Salicaria. 8. M. cinerea, fubtus alba, fuperciliis albis.

Lufcinia falicaria. Klein. ati. 74.

Salicaria Gefneri. Will. orn. 158. Rai. all. 81. Alb. au. 3. p. 56. t. 60.

Sylvia

Habitat in Europa.

Syluia. 9. M. fupra cinerea, fibtus alba, rętrice prima longitudinaliter dimidiato alba, fecunda apice alba. Fn. fuec. 228.

Habitat in Europa.

Philome- 10. M. fupra grifea, fubtus cinerea, remigibus primoribus la. apice obfoletis.

Iufcinia altera. Klein. au. 73. t. x.

Habitat in Europa.

Ficedula. Ir. M. fubfufca, fubtus alba, pectore cinereo maculato. Fn. fuec. 23r.

Ficedula cannabina. Will. orn. 163. Rai. all. 8I. $n$.

12. Alb. alt. 3. p. 25. t. 26.

Habitat in Europa.

alba. 12. M. pectore nigro, rectricibus duabus lateralibus dimidiato oblique albis.

Motacilla pectore nigro. Fn. Juec. 214 .

Motacilla. Gefin. au. G18. Aldr. ornith. l. 17. c. 23. Bell. au. 88. 6. Will. orm. 171. t. 42. Rai all. 75 . n. I. Alb. au. I. p. 49. to 49. Frijch. all. . t. 23. f. 4 . Olin. all. 43 .

Hibitat in Europa.

flaua. 13. M. pectore abdomineque flauo, rętricibus duabus lae teralibus dimidiato oblique albis. Fn. Jüer. 215 . 
Motacilla flaua. Gefn. au. 6r8. Aldr. 0rn. l. I7. c. 24. Will. onn. 172. t.68. Rai. au. 75. n. 2. Alb. au. 2. p. 54. t. 58. Frifch. au. . t. 23.f. 3 .

Habitat in Europa.

Tiphia. I4. M. viridis, fubtus flauefcens, alis nigris: fafciis duabus albis.

Paffer viridis indicus. Edw. au. 79. t. 79.

Habitat in Benghala.

Fus ciace alarum ex apicibus tectricum albis.

Ruticilla. I5. M. nigra, pectore macula alarum bafique remigum reetricumque fuluis.

Motacilla americana. Catesb. car. r. p.67.t.67.

Serino affinis auicula e croceo et nigro varia. Sloan. iam. 2. p. 312. Rai. au. I8S. n. $5 \mathrm{I}$.

Ruticilla minor americana. Edw. aw. 80. t. 80.

Habitit in America.

hifpanica. I6. M. ferruginea, area oculorum alis caudaque fufcis, reetricibus extimis latere albis.

Oenanthe fulua. Edwo. au. 3I. t. 3 I.

Habitat in Hifpania.

Oenan- I7. M. dorfo cano, fronte alba, oculorum fafcia nigra the.

Fn. Suec. 217.

Oenanthe 1. Vitiflora. Ionft. au. 123. t. $45 \cdot f$. I3. Will. orm. Í6. t. 41. f. 4. Rai. au. 75. n. I. Alb. ail. I. p. 53. t. 55 .

B. Motacilla fubtus pallida, reetricibus introrfum albis, dorfo vndulato. Fn. Juec. 219 .

Oenanthes vitiflorae femina. Alb. au. 3. p.50.t. 54 . Habitat in Europae apricis lapidofis.

Sexu et aetate varia; cel]ante noctumo frigore aduenit.

Rubetra. I8. M. nigricans, fuperciliis albis, macula alarum alba, gula pectoreque flauefcente. Fn. Suec. 218. et 230 . t. 1. $f: 3,4$.

Mufcicapa f. Oenanthe tertia. Aldr. om. 735. Rai. au. 76. n. 4. Will. orn. 169. t. 41. f. 5:

Curruca maior altera. Frifch. alt. . t. 22. f. 2.

Ruberra. Alb. alm. I. p. 50. t. 52 .

Ficedula f. Atricapilla alia. Ionft. au. . t. $45 \cdot f$. vit. Ficedula 3. aldrouandi. Edw. aw. 30.t. 30.

Habitat in Europa. 


Atrica. Ig. M. teftacea, fubtus cinerea, pileo obfcuro. Fn. fuec. pilla.

Atricapilla f. Ficedula. Aldr. orn. l. 17. c. 36 . Will. om. 162. t. 41. Rai. au. 78. n. 8. Frifch. au. t. 24. f. I. Olin. aur. 9 .

Habitat in Europa.

Emeria. 20. M. grifea, fubtus alba, temporibus vropygioque rubris.

Ruticilla bengalenfis. Edw. au. 190. t. IgO. Alb. am, 3. p. 52. t. 56 .

Habitat in Benghala.

Phoeni- 2I. M. gula nigra, abdomine rufo, capite dorfoque cano, curus. Fin. filec. 224 .

Ruticilla. Will.0\%m. 159. Rai. au.78. 17.5. Alb. au. I. p. 48. t. 50 .

Phoenicurus media, penna caudae fubnigra. Frifch. an. t. 20. $f \cdot 3$.

Habitat in Europa.

Eritha- 22. M. dorfo remigibusque cinereis, abdomine ręricicus. busque rufis : extimis duabus cinercis. Fn. fuec. 225. Habitat in Europa.

Titys. 23. M. remigibus nigricantibus, tęricibus rufis: intermedio pari nigro extrorfum rufefcente. Fn. ficc. 227. Habitut in Europa.

fuecica. 24. M. pętere ferrugineo fafcia caetulea, rętricibus fufcis verfus bafin ferrugineis.

Motacilla pectore caerulco: macula flauefcente albedine cincta. Fin. Juec. 220.

Wegflecklin. Gisin. au.796. Aldr. orn.749. Will. orn. 160. Rai. all.78.

Ruticilla gutture caeruleo. Edvo. au. 28. t. 28.

Phoenicurus pectore caeruleo. Frijch. au. 3. t., I9. f. 3.4 .

B. W'ydengyckerlin. Giefin. au. 796.

Habitat in Europue alpinis.

Sialis. 25. M. fupra caerulea, fubtus tota rubra.

Rubecula dorio caerulco. Catesb. car. I. p. 47. t. 47? fillw. all. 24. t. 24 .

Ilabitat in Bermudis ot America calidiore. 
Velia. 26. M. caerulea, ventre clunibusque rufefcentibus. Mufcicapa caerulea, ventre rubro. Ediv. au. 22. t. 22.

Spiza.] 27. $M$. viridis, remigibus primoribus nigricantibus.

Mufcicapa viridis, capite nigro. Edw. au. 25. t. 25. $f . \mathrm{I}$.

B. Mufcicapa viridis, capite caeruleo. Edw. au. 25. $t$. $25 . f \cdot 2$.

Habitat Surinami.

Rubecus- 28. M. grifea, gula pectoreque fuluis. Fn. fuec. 226.

la. Rubecula. Will. orn. 160. t. 38. Rai. au. 78. n. 3. Alb. aus. I. p. 49. t. 51. Frifch. «u. 3. t. 19. f. 2. Olin. au. 16.

Habitat in Europa.

Troglo- 29. M. grifea, alis nigro cinereoque vndulatis. Fr. fuec. dytes.

Paffer troglodytes. Gefin. au. 651. Aldr. om. l. 17. c. 4. Will.orn. 164.t. 42. Kai. au. 80. Alb. au. I. $t$. 53. $f$. $B$.

Trochilus remigibus teffellatis. Frifch. au...t.2. 4. f. 3 .

Habitat in Europa.

Regulus. 30. M. remigibus fecundariis exteriori margine flauis, medio albis. Fn. Jieec. 235 .

Regulus criftatus. Will. orn. 163. t. 41. Rai. au.79. n. 9. Alb. au. I. p. 51. t. 53. Aldr. orn. l. 17.c. I. Frifch. au. . t. 24. f. 4. Catesb. car. 3. p. 13. t. I3.

Habitat in Europa.

Trochi- 31. M. cinereo-virens, remigibus fubtus flauefcentibus, lus. fuperciliis luteis. Fn. fitec. 236.

Regulus non criftatus. Aldr. orn. l. 17. c. 2. Will. orn. 164.t. 42. Rai. an. 80. 13. 10. Alb. au. 2.p. 55. t. 59.f.A. Frijch. .. t. 24.f. 2.

Habitat in Europa.

Conf. Locuftella. Will. orn. I5I. Alauda mininua lo. cuftue voce. Rai. au. 70 ; pro eadem babet. Muf. petrop. 380. 


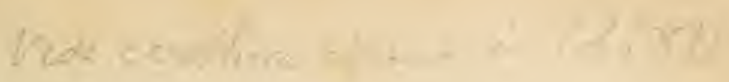


no34. Pipra mimnta $\therefore 1$ 64. lb. A. F.p.34.-

wo Paruan P.1935. 
Acredu- 32. M. fitpra cinereo - virefcens, remigibus fufcis: feptimo

l.. octauo nono apice allis. Fn. Jilec. 237.

Habitat in Europa.

Penduli- 33. M. capite ferrugineo, macula nigra oculari, remigibus nus. re?tricibusque fufcis margine vtroque ferrugineis.

Lanius minimus.' $E d w$. cul. $55 . t .55$.

Parus montanus. Alb. alu. 3. p. $53 . t .59$.

Parus minimus Remiz. Titius Lipf: 1755. difertatio.

Parus lithuanicus nidum fufpendens. Klein. an. 86.

Pendulinus. Act. Bonon. 2.2. P. 57.t. 7 .

Remiz. Rzaczynfr. polon. I. p. 294 .

Habitat in Polonia, Lithuania, Hungaria, Italia, miffe a D D. Montio.

minuta. 34. M. grifea, capite nigro punctis albis fparfis. Muf. Ad. Fr. 2. p...

Habitat in India.

Corpus magnitudine Reguli. Dorfum Alaeque fippra grifea. Pectus flaneficens lineis transucr fis nigris. Cauda filfca, lateribus pallida. Caput nigrum, adfperfinm punitis rotundis albis, in fingula pema Jingulis.

Mas antice in capiie lineis carneis, poftice punstis albis.

100. PARUS. Rofrum integerrimum.

Lingua truncata, fetis terminata.

criftatus. I. P. capite criftato. Fn. fuec. 239.

Parus criftatus. Gefin. att. 642. f. med. Aldr. orn.l. 17.6. 1 20. Will. orn. 175. t. 43. Rai. anl.74. n. 6. Alb.au. 2. p. 53.t. $57 t .2$. Frijcl. au...t. I4.f. 2 . Habitut in Europa.

inajor maior.

2. P. capite nigro, temporibus albis, nucha lutea. Fn. fuec. 238.

Parus maior. Giefn. au. 640 Bell. all. 95. a. Aldr. orn. I. 17. c. 13. Will. orn. 174. Rai. all. 73. ". 3. Alb. all. I. p. 44. t. 46. Irijacb. aw. 3. t. I3. $f$. I. 2 . Olin. an. 2S.

Habitat in Europa. 
america- 4 . P. caerulefcens, temporibus pectore dorfoque flauefcennus. tibus, hypochondriis purpurafcentibus.

Parus Fringillago. Catesb. car. 1. p. 64.t. 64 .

Habitat in America Jeptentrionali; an jola varietas Pari muioris.

caeruleus. 4. P. remigibus caerulefcentibus: primoribus margine exteriore aibis, fronte alba, vertice caeruleo. Fn. fiuec. 240.

Parus caeruleus. Bell. au. 96. a. Gefin. au. 64I. Aldr. orn. l. 17.c. 17. Will. om. 175. Frifch. all. 3.t. I4. $f$. I.

Habitat in Europa.

ater. 5. P. capite nigro, dorfo cinereo, occipite pectoreque albo. Fin. fitec. 241.

Parus ater. Gefn. an. 94I. Aldr. om. l. 17. c. 19. Will. orn. 175. t. 43. Rai. au.73. n.2. Frifich. au. Habitat in Europa.

paluftris. 6. P. capite nigro, temporibus albis, dorfo cinereo. Faun. fuec. 242 .

Parus paluitris. Gefn. an. 642. Aldr. orn. l. I7.c. I8. Will. orm. 165. t. 43. Rai. au. 73. n. 3. Alb. au. 3. p. 54.t.58. Frifch. au. 3.t. 13.f. 4 .

Habitat in Europa.

caudatus. 7. P. vertice albo, cauda corpore longiore. Fn. Suec. 243 . t. I. $f$. 243 .

Parus caudatus. Gefn. an. 642. fig. infina. Aldr. orr. 1. I7. c. I5. Will. orn. 176.t. 43. Rai. all.74. 12.8. Alb. alu. 2. p. 53.t. $57 \cdot f \cdot 1$.

Habitat in Europa.

biarmi- 8. P. vertice cano, cauda corpore longiore.

cus. Parus beardmanicus. Alb. au. I. p. 46.t. 48 .

Lanius minimus. Edwo. alt. 55.7 .55 .

Habitat in Europa.

Pipra. , 9. P. ater, capite fupra albo. Muf. Ad. Fr.2. p.

Auicula toto corpore nigra, cum vitta alba. Seb. muj. 2. p. 102. t. $96 . f \cdot 5$.

Ilabitat in Indiis. 


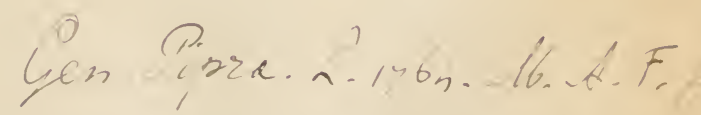

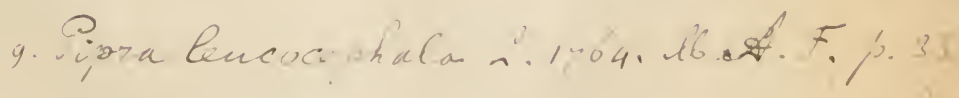




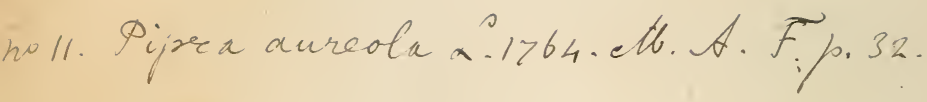

no 12. Pipra lencocilla? R.1764. Ab.A. F. p.33.

101. Boinindo 21735 
Statura Motacillae, finilis fequenti, fed roffrum paulo validius. Corpus totum nigrum. Cuput fotmin album. Vibriflae ad os, validiores quam in congeneribus.

erythro- 10. P. niger, capire femoribusque fuluis. cephalus. Parus auricapillus. Kiein. anl. $\$ 6$.

Parus niger, capite fuluo. Edw. au. 2I. p. 2t.

Habitat in America auffrali.

Aureola, tr. P. niger, capite peîroreque coccineis, remigibus antrorfum macula alba. Muf $A d$. Fin. 2. p.

Parus niger et fuluus, Eulwo all. 83.t.83.f.2.

Auicula furinamenfis nigra, capite e luteo coccineo, Pet.gaz. 73.t. 46.f: Iо.

Auicula mexicana de Chichil totolh altera. Seb. muf.

I. p. $96 . t .60 . f .8 \cdot 7$.

Habitat in America.

Cela 、 I2. P. niger, roftro albo, macula alarum bafique caudac flauis. $M u l$ : Ad. Fs. 2. p. .

Habitat in Indiis.

101. HIR VND O. Roftrum minimum, incurulim, fil bulatlim; bafi depiefium.
Rictus capite amplior.

ruftica. I. H. reetricibus, exceptis duabus intermediis, macula alba notatis. Fin. Jilec. 244 .

Hirundo domeftica. Giefir. au. 548. Aldr. orn. 1. 17. c. 6. Ionft. all. II j. t. 42. Will. orn. 155 t. 39 . Rai. au. 7I. n. I. Aib. au. I. p. 4j.t. 45. Frijch. au. t. I8.f. I.

Habitat in Eurnpae domibus intra teifum.

Ruflica et vrbica autunno demergitur, vereque emergit.

efculen- 2. H. rectricibus omnibus macula alba notatis.

ti.

Hirundo nidis edulibus. liont. iatl. 66. Rai. att. 72.

Capus marina. kisuph. herl. 6. p. 183. to 75. f. 4 . Olear. muf:: 25. t. 14. f. 2. 6.

Habitat in China ad rifias, midis gelatinofis efculentis. $\mathrm{N}_{2}$ 3. H.

Hirundincs in gëre pifcantur infestn. 
vrbica. 3. H. rectricibus immaculatis, dorfo nigro-caerulefcente. Fr. Juec. 245 it. oel. $4 \mathrm{I}$.

Hirundo agreftis r. ruftica. Gefn. an. 564. Aldr. onn. l. 17. c. 7. Will. orn. 155. Rai. au. 71. 21.2. Alb. au. 2. p. 52. t. 56. f. A. Frijch. au. 3.t. 17. f. 2 .

Habitat in Europa, ad parietes fub tecto.

$V$ enit venit Hirindo, pulchra tempora adducens et pulchros annus. Hejych.

riparia. 4. H. cinerea, gula abdomineque albis. Fn. fuec. 247.

Hirundo riparia f. Drepanis. Gefin. aut. 656. Aldr. orn. 1. 17. c. 8. Will. orn. 156. t. 39. Rai. an. 71. $n$. 3. Alb. au. 2. p. 52. t. 56. f. B. Frijch. au. t. I8. $f$. 2 .

Habitat in Europae collibus arenofis abruptis, foranine firpentino.

Apus. 5. H. nigricans, gula alba, digitis omnibus quatuor anticis.

Hirundo tota nigra, gula albicante. Fn. fuec. 246 .

Hirundo Apus. Bell. au. 100. a. Gejn. au. 166. Aldr. orn. l. I7. c. Iо. Ionft. au. II9. t. 42. W'ill. orn. 156. t. 39. Rai. au. 72. n. 4. Alb. au. 2. p. 5 i. t. 55. Frijch. au. 3. t. I7. f. I.

Habitat in Europae altis.

In terram decidens non euolat; capitur bano Cicadae inferto. Bell. I6.

Subis. 6.H. nigro-caerulefcens, ore fubtusque cinereo-exalbida.

Hirundo caerulea canadenfis. Ediv. all. I20. t. I20 Habitat ad finum Hudfonis.

pelagica. 7. H. reetricibus aequalibus apice fubulatis nudis.

Hirundo, cauda aculeata, americana. Catesb. car. 3.p. 8.t. 8 .

Havitat in America.

Melba. 8. H. fufca, gula abdomineque albis.

Hirundo riparia maxima. Edw. au. 27.t.27. Klein. au. 83 .

Habitat ad fretum Herculeum. 

v2 Capsimmlgero Lisso. 
102. CAPRIMVLGVS. Rofrum incuruum; minimum, fubulatum, bafi depreffum.

Vibriffae ad os ferie ciliari.

Rictus amplifímus.

euro- I. C. narium tubis obfoletis.

paeus. Hirundo cauda integra, ore fetis ciliato. Fn. Juec. 248.

Caprimulgus. Bell. ant. 28. a. Gefn. aur. 242. Ionft. au. 53.t. 20. Will. orn. 70. $t$. 14. Rai. au. 26.n. 1. Alb. all. 1. p. 10. t. 10. Frifich. au. . t. 101. Catesb. car. I. p. 8. t.8.

B. Caprimulgus minor americanus. Catesb. car. 3. p. 16 . t. 16. Edvo. au. 63. t.63.

Habitat in Europa et America.

Varietatem $\beta$. iudico ex macula alba alarum et rectricum, wt in mare auis europaeae.

Victitat Pbalaenis et infectis nocturnis. Nidus orbiculatus e lato inter rupes.

2merica- 2. C. narium tubulis eminentibus.

nus. Caprimulgus $\mathrm{f}$. Noctua fyluatica iamaicenfis. Sloan. iam. 2. p.298.t.255.f. I. Rai. au. 180 .

Hirundo maior fubfufca mifcella, macula alba fphaerics in vtraque ala. Brown. ianr. 467.

Habitat in America calidiore.

$\mathrm{N}_{3}$

CLAS.

Caprimulgus genere differt ab Hirundine, vti Strox a Falcone, Phalaena a Papilione. 


\section{$C L A S S I S$ III. \\ A $M \cdot P$ P H I B I A.}

PESSIM A tetraque Animalia Corde vniloculari vniaurito, $P_{u l-}$ monibus arbitrariis, Pene duplici dignofcuntur.

AMPHIBIA pleraque horrent Corpore frigido, Colore lurido, Sceleto cartilagineo, Cute foeda, Facie torua, Obtutu meditabundo, Odore terro, Sono rauco, Loco fqualido, $V$ eneno horrendo: non itaque in horum numerum fefe iactauit eorum Auctor.

POLYMORPHA in his Amphibiis natura duplicem vitam plerisque conceffit: Aliis Metanurplofin fubire, aliis Senectan exuere; aliis $O u o$ nafci, aliis mudos foetus parere; aliis in ficco bunidoque promifcue viuere, alis dimidio anno byemare; aliis praedam nifu altuue affequi, alis fafcino quali eandem in fauces reuocare.

REPTILFS pedati, auribus plano-denudatis absque auriculis, variam vitam ex ftructura agunt: Teftudines muniuntur Tefta; Dracones euolant Alis; Lacertae fugiunt Pedibus; Ranae abfcondintur Lnco; nec ommes Veneno prorfus carent, vt Bufo, Saiamandra, Gecko.

SERPENTES apodes, absque Awribus furdos, a Pifcibus feparant Pulmones, Ounrum catena, Penisque duplex muricatus, affinitas denig̨ıe cum Lacertis tanta, ac Lacertarurn cum Ranis, virque limites admittens. Hos nuda in terra reiectos, arfuum minifterii expertec, omnium iniurne expofitos, armuit Narmra conferuntrix fisis avmis, horrentibus exfecrabli Veneno, in diuerlis diuerfo. Seme 
Amphibia L. ngs.

Reptilia riYo4. - lo. A. F. p.35.

derpentia A. 1764 . W. H.F., 38 
Naniria A. 1904.- M. At.F.p.35. 
autem haec Tcla dentibus fimillima, fed extra maxillam fuperiorem collocata, proque lubitu exferenda et retrahenda, facculo faniei affixa, quam vulnere inflant in fanguinem, tum diri effectus cauffam, caeterum inertem; ficque Catonia haec morfu virus habent et fatum dente minantur, pocula morte carent, prout folide liedi. Ne vero hi fpoliati miferique armis, quae ipfis fupereffent, nimium faeuirent, decimam quamque tantum fpeciem armauit ( $\sigma^{\lambda}$ ) Imperans, fed verfipelles eos voluit, vt dubii omnes metuerentur ab omnibus; at Beneficus Homini dedit INDIS Ichneumonen cum Opbiombiza, AMEricaivis Suem cum Senega, evropaeis Ciconiam cum Olea.

DIAGNOSIN, qui velit horum, eandem defumat a Pedum fuccedaneis Scutis abdominalibus caudalibusque; ne vero numerus alteri demtus alterique additus feducat, iuuat etiam vterque connumeratus. (ACt. Stockh. 1752. p. 206.); ab ano vtrinque Longitudo illuftrat, et Color in nonnullis; caue vero ne renata fit mutilata cauda.

NANTES pinnatos aquaticos (Chondropterygios cartilagineosue' dictos Pifces) Amphibiorum claffem nunc fubire iubent Pulmones $\left({ }^{*}\right)$ arbitratii, Latere licet, non vero branchiis liberis, fed adnatis, refpirantes; Maresque Pene duplici infidentes Feminis! ouis concatenatis foctis, foedaque Cutis, Offaque cartilaginea, cetera. Neque hi omnes Toxico expertes, tefte Paftinaca, Torpedine.

A MPH ID I O L O G I omnium pauciffimi funt, nullique veri.

Seba numero ftupendo fibi ignota pulchre collegit et delineauit, fed multiplicauit, minimeque defcriplit. Catesbaçus nec paucos Serpentes pulchrius delineauit, quam notauit.

(“) I'vlmones hormm fectinati vt l'ifcium, fed adnati vafi arcato cylindrico tubslofo, absque radio ofjco, ncc pificm fimili, nifi externa fisura. 


\section{GENERVM Characteres.}

I. REPTILES pedati, fpirantes ore:

I03. TESTVDO Corpus Tefta munitum.

104. DRACO Corpus Alis volatile.

105. L ACERTA Corpus (Tefta Alisuc) nudum, caudatum. 106. RANA Corpus fimiliter mudum, ecaudatum.

II. SERPENTE $S$ apodes, fpirantes are:

107. crotalvs Scuta Abdominalia Caudaliaque cum Crepitaculo.

I08. во A Scuta Abdominalia Caudaliaque absque Crepitaculo.

109. Colvber Scuta Abdominalia; Squanae Caudales. IIO. ANGVIS Squamae Abdominales Caudalesque.

III. A mpнis в A Na Annuli Abdominales Caudalesque. II2. CAECILIA Rugae mudae laterales.

III. NANTES pinnati, fpirantes lateribus:

II3. PETROMYZON Spiracula 7.

II4. RA1 A Spiracula 5 fubtus.- Corpus depreffum.

II5. Seva lvs Spiracula 5 lateralia. Corpus teres. II6. сн і ма ев A Spiracula I. Dentes primores incifores 2. II7. цорніvs Spiracula I. Pinnae pectorales bracbio injertae.

II8. ACIPENSER Spiracula I. Os retractile. 
Reptilia 21 194.- b. A. F. p.35=

Lerpentia L.1764.- Llo. L F. F. 85.

Nantix a.1764-No. A-F.p..35:

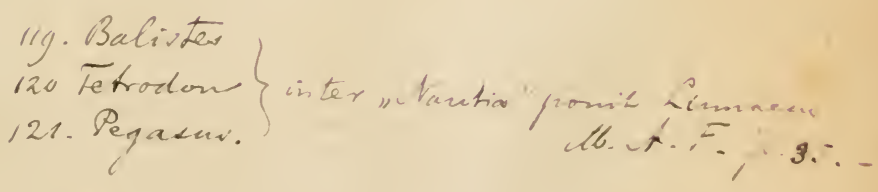





\title{
I. REPTILES.
}

\author{
Os refpirans. \\ Pedes quatuor.
}

103. TESTVDO. Covfus tetrapodum, caudatum, tefta obtectum.

Mydas. T. pedibus pinniformibus, vnguibus palmarum binis, plantarum folitariis, tefta ouata.

Amocn. acad. I. p. 138. Teftudo vnguibus acuminatis:

palmarum duobus, plantarum vnico.

Mut. Ad. Fi. I. p. 50. Teftudo atra.

Osb. iter. 293 .

Gefir.quadr.78. Teftudo marina.

Aldr. quadr. 712. t. 714.

Grew. mut: 38.t. 3. f: 4 .

Olear. mul. 27. t. 17. $f$. I.

Bradl. natur. t. 4. f. 4 .

6. Scb. muf: i. t. so.f: $q$. Teftudo marina americana, Mydas dieta.

Amoin. acad. I. p. 137. Teftudo eadem.

Marcgr. braf: 24I. Iurucuia brafilienfibus.

Rai. quadr. 256 .

\%. Amoen. aced. I. 1.287. 21.7. Teftudo vnguibus acuininatis, palmarum plantarumque folitariis.

Muj: Att. Fr. I. p. 50. Telludo eadem.

Seú. mut.: r. t. 79. f. 4. 5. 6.

Habitat ad infulas Pelagi: infulam Adfcenfionis cte. oura membranacea noctu in Arena calcaria rotunda defodit.

Caro viridefcens, efculenta; refspinata in mari dormiens natat; incedit tot bominibus, quot ei infiflant; in terra refupinata non erigitur. Tcfta olim pro fó tis, formicibus.

Caretta. T. pedibus pinniformibus, vnguibus palmarum plantarumque hinis, teftal ouata acute ferrata.

Gron. mus.2. p. $55 \cdot n \cdot 69$. Teftudo pedibus natatoriis, vngublus acunisatis binis.

Brovon. iam. 465. Teftudo vnguibus vtrinque binis acutis, figuamis dorfi quinque gibbis. 
Catesb. car.2. p.39. t. 39. Teftudo Careta Rochef. Rai. quadr. 258. Teftudo Caretta. Habitat ad injiulas Americanas.

orbicula- 3. T. pedibus palmatis, tefta orbiculata planiufcula. ris. Rai. quadr. 254 . Teftudo aquarum dulcium?

Habitat in meridionalibus Europae.

Tefta parua integerrima orbiculata, nec antice nec poflice emarginatı. Sternum puftice bifidum. Pedum digiti membrana comexi in plantam orbiculatam.

fcabra. 4. T. tefta planiufcula, fcutellis omnibus intermediis dorfacis.

Habitat in Indiis.

Corpus ct teflae latera fubtus albo nigroque varia: Stermunn antice truncatum.

lutaria. 5. T. pedibus fubpalmatis, cauda corpore dimidio breuiore, tefta fubconuexa poftice tribus fcutellis carinata.

Amuen. acad. I. p. I39. n. 23. Teftudo vnguibus acuminatis, palmarum plantarumque quaternis. *

Habitat in Italia, Oriente.

Palmae magis palinatae, plantae minus. Stermum pofice truncatum.

graeca. 6. T. pedibus fubdigitatis, tefta gibba margine laterali obtufiffimo, fcutellis planiuculis.

Teftudo teffellata minor africana. Edw. au. 204. $t$. 204 .

Habitat in Africa.

Corpus caudatum, palnis snguibus 5 , plantis vero 4 .

carolina. 7. T. pedibus digitatis, tefta gibba, cauda nulla.

Teftudo teffellata minor caroliniana. Edw. au. 205. $t$. 205 .

Habitat in Carolina.

Palmae pentadactylae, Plantae tetradactylae.

carinata. 8. T. pedibus digitatis, tefa gibbofa, fcutellis dorfalibus quattor anterioribus carinatis, fterno integro.

Habitat in Calidis regionibus.

9. $T$.

Teftudines abfiffo copite facpe 14 dies viuunt; byeme in frigidioribus bybernant terreftres. Vullum animal Teftudine tardius; copula cobaerent faepe per menfem. 


geome- 9. T. pedibus pofticis palmatis, teftae fcutellis eleuatis trica. truncatis.

Muf. Ad. Fr. I. p. 50. Teftudo vnguibus acuminatis: palmarum 5, plantarum 4.

Worm. muf: 317 . Teftudo pi\&a f. fellata.

Amoen. acad. I. p I39. n. 24.

Grew. muj: 36. t. 3. f. I, 2. Teftudo tefta tefiellata maior.

Seb. mil: I. $t$. 8o.f. 8. Teftudo minor amboinenfis. Pif braf. t. 105. Teftudo nigricantibus et flauefcentibus figuris geometricis.

Rai. quadr. 259. Teftudo teffellata minor.

Habitat in Alia.

Tefta nigra foutellis flauis radios paruos anaftomofantes enittentibus.

pufilla. Io. T. tefta hemifphaerica: fcutellis conuexis trapeziis margine frratis difco punetati.

Grem. mul. $38 \cdot t \cdot 3 \cdot f \cdot 3 \cdot$ Teftudo virginea.

Worm. mul: $3 \mathrm{I} 3$. Tefudo terreftris pufilla ex India orientali.

Rai. quadr.25\%. Teftudo eadem.

Habitat in India.

ferpenti- II. T. pecibus digitatis, tefta fubconuexa poftice obtufa na. acute quinquedentata.

Habitat in Calidis regionibus.

104. DRACO. Corpus tetrapodum, caudatum, alatum.

volans. I. DRACO. Syft. nat. 36. Gron. muf. 2. p. 73.n. 46. Muf. Ad. Fr. I. p. 40. Draco.

Amocn. acad. I. p. I26. Lacerta cauda tereti, pedibus pentadactylis, alis femore connexis, crilla gulae triplici. Bont. iant. 57. t. 57. Lacerrus volans f. Dracunculus. Seb. mul: 2. t. 86. $f$. 3. Lacerta africana volans f. Dra. co volans.

Bradl.

Tefudixibus Pelum digiti untici s, pefici f, fotidem :nguilus, cxceptis marinis pinniformibus pedibus siuta tefac numero fapius conueninnt, znde diagrofis Specierun dif crilema

Draccones omves reliqui anctorm fibling funt, et HYDRA, Seb. mu? t. 102. f. 2. Hamburgi a nobis vifa, non naturae, Jed artis opes eximinm. 
Bradl. natur. t. 9. f. 5. Lacerta volans.

Habitat in India, Africa.

105. LACERTA. Corpus tetrapodum, caudatum, nudum.

* Cauda compreffa.

Croco- I. L. cauda compreffa, pedibus triunguiculatis, palmis dilus. pentadactylis, plantis tetradactylis palmatis. Amven. acad. I. p. I2I. Lacerta eadem. Muf. Ad. Fr. I. p. 40. Lacerta eadem.

Grun. muf.2.p. $74 \cdot n \cdot 47$. Crocodilus maxillis depreffis conicis, pedibus etc.

Bellon. aquat. 4I. Crocodilus.

Gejn. quadr. 9. Crocodilus.

Aldr. aquat. 677. Crocodilus.

Ionift. quadr. t.79.f. 3. Crocodilus.

Rai. quadr. 26I. Lacertus maximus.

Bont. iau.t. 55. Crocodilus Cayman.

Marcgr. braf. 242. Yacare.

Olear. muf: 8.t.7.f. 3. Crocodilus.

Beli. mus. 47.t. 13. Crocodilus niloticus.

Sloan. iam. 2. p. 332 . Crocodilus.

Seb. mif. I. t. 103. IO4 $_{4}$ Crocodilus.

Merian. Jiır. 49. $f .69$. Crocodilus.

Vallifin. nat. 1. t. 43 .

Catesb. car. 2. t. 63. Lacertus maximus.

Habitat in Africae, Aliae, Americae Aquis.

Anatome Crocodili. Hafelq. iter. 292.

Oua centena deponit, effodiuntur ab Icbneumone, auicula; exclufos pullos Mater in tergum prourocat, ad aquas ducit, deciduos Mas Feminaque ingurgitant; fub aqua vorat; in catenam adfociati aquatilia exagitant. Leuiatban Iobi. 40: 20; 41:24.

Iugientibus atrox; edocti dorjo ipfius infiliunt, regunt.

Caudiuer-2. L. cauda depreffo-plana pinnatifida, pedibus palmatis. bera. Feuill. permu. 2. p. 3I9. t. 319.

Sel. mul:2. t. 62. f. 9 .

Habitat in Peru.

fuperci- $3 . \mathrm{L}$. cauda carinata, dorfo fuperciliisque ciliatis. liof. Muf. Ad. Fr. I. p. 40. Lacerta eadem. * 




\section{AMPHIBIA REPTILIA. Lacerta.}

Seb. muf. I. t. Iog. f. 4 .

Habitat in Indiis.

fcutata. 4. L. cauda fubcompreffa mediocri, futura dorfali dentata, occipite bimucronato.

Scb. mnil: I. p. 173. t. Iog. $f \cdot 3$. Salamandra prodigiona amboinenfis fcutata.

Habitat in Afia.

Monitor. 5. L. cauda carinata, corpore mutico.

Muf: Ad. Fr. I. p. 4I. Lacerta cauda ancipiti integra, pedibus pentadactylis, digitis omnibus vnguiculatis. * Seb. mul:2.t. 86. f: 2 .

t. $10 ; . f: x$.

Habitat in Indiis.

I. $t .94 \cdot f \cdot 1.2$.

Corpus maculis albis ocellatis verticillatum. Fafciae abdominis albi lineares interruptae.

principa- 6. L. cauda fubcarinata, crifta gulae integerrima, dorfo

lis. laeui.

Mirf: Ad. Fr. I. p. 43 .

Anoen. acad. .. p. 286. t. I4.f. 2.

Habitat in Indiis.

bicarina- 7. L. cauda compreffa fupra bicarinata mediocri, dorfo

ta. quadrifariam-carinato - Ptriato.

Habitat in indiis.

Parua, grifea. Dorfum ftriis duabus elenatis et latere dorfi fingula ex finamis carinato-Jtriata; Latera fquanis conuexo-tube:cu!atis. Abdomen 24. ordinibus transuerfalibus filuamarum (in jingulo 6.) tectum. Cauda corpose :iv Jefinilongior, cumpreffa; jisbtus ftriatu, lateribus lacuis, fupra duplici cariua angulata. Crifta mulla.

paluftris. 8. L. cauda ancipiti mediocri, pedibus muticis fifis; palmis tetrad cetylis. Inz. Juec. 256 .

Gion. mul: 2. p. $77 \cdot n \cdot$, r. Salamandra alepidota verrucoli etc.

Rai. quadr. 273. Salamandra aquatica.

Seb. murf." r. t. 89. .". 4. 5. Lacerta americana.

Habitut in Furopae aquis dulcibus ftagnantibus. An difling a jpecies o L. "gili, *ol larwa alius? 
* * Courla verticillata.

Cordy- 9. L. cauda verticillata breui: fquamis denticulatis, corpolus. re laeuigato. Amoen. acad. I. p. Iวิ1. 292. Gron. mul: 2. p. 79. n. 55. Cordylus. Seb. mut. I. t. $84 \cdot f \cdot 3 \cdot 4$. et 2.t.62.f. 5 . Habitat in Africa, Afia.

Corpus fquamis truncatis verticillatum.

Stellio. Io. L. cauda verticillata mediocri: fquamis denticulatis, capite corporeque muricato.

Haffelq. iter. 30I. Lacerta Stellio.

Tournef. itin. I. p.IIg. t. I20. Lacerta Coflordilos dieta. Seb. muf. 2.t. 8.f.7.

Habitat in Oriente: Delos, Aegypto, Africa.

maurita- II. L. cauda fubuerticillata breui muricata apice laeui, corpore nica. fupra muricato, pedibus fubtus fquamatis.

Habitat in Mauritania. E. Brander.

Corpus babitu et figura L. Gecko, fed luridum et ad latera capitis, fupra collum, dorfirm, fenora, verrucofism mucronibus. Cauda corpore breuior, a bafi ad medium Jpinis fexfarianm muricata, inde ad apicems lacuis. Digiti pedum ommino vt in Gecko fubtus lamellofo-fquanuati, onguibus minimnis. Subtus totum corpus laeue fquamis minimis.

azurea. I2. L. cauda verticillata breui: fquamis mucronatis.

Muf. $A d$. Fr. I. p. 42. Lacerta eadem.

Seb. mul: 2. t. 62.f. 6 .

Habitat in Africa.

turcica. I3. L. cauda fubuerticillata mediocri, corpore grifeo fubverrucofo.

Edw. au. 204. t. 204. Lacerta minor cinerea maculata afiatica.

Habitat in Oriente.

Cauda vix corpore longior, obfolete verticillata. Corpus paruum, grifeum, punctis fuccis irroratum, inaequale et quafi verrucis obfoletis adfperfun.

Ameiva. I4. L. cauda verticillata longa, fcutis abdominis triginta, collari fubtus ruga duplici.

Amoen. acad. I. p. 127. 293. Lacerta cauda tereti corpore duplo longiore, pedibus pentadactylis, crifta nulla, fcutis abdominalibus 30 . 


Mill. Ad. Fr. I. p. 45. Lacerta eadem.

Gron. muf. 2. p. 80.t. 56. Lacerra cauda tereti corpore triplo longiore, fquamis lacuifimis, abdominalibus oblongo - quadratis.

Clul: crot. II5. Lacertus indicus.

Edw. an. 202. t. 202, 203. Lacertus maior viridis. Womm. mis. $313 \cdot f$ : 313.

Rai. quadr. 270. Lacertus indicus.

Scb. mul: I. t. $85 . f: 2,3$.

$$
\text { t. } 88 . f .1,2 .
$$

Sloan. iam.2. p. 333.t. 273. f. 3. Lacertus maior cinereus maculatus.

B. Amocn. acai. I. p. I3O. Lacerta cauda tereti corpore fefquilongiore, pedibus pentadactylis, dorfo lineis longitudinalibus friato.*

Habitat in America.

agilis. I5. L. cauda verticillata longiufcula fquamis acutis, collari fubtus fquamis conftructo.

Fnun. Juec. 1352. Lacerta cauda tereti verticillata longitudine corporis, pedibus pentadaftylis vnguiculatis. Syft. nat. 36. n. 6. Lacerta cauda tereti longa verticillata fuumis acutis, pedibus penradactylis vnguiculatis. Muf: Ad. Ir. r. p. 43. Lacerta eadem.

Gron. mul: 2. 1). 80 n. 57. Lacerta cauda corpore parum longiore, fiuamis laevifimis.

Rai. quadr. 264. Lacertus vulgaris, ventre nigro maculato.

Scb. mul: 2.t. 79. f. 4. Lacerta minor maculata indigena.

B. Rai. quadr. 264. Lacertus viridis.

Aldr. quadr. 634. Lacertus viridis.

Seb. mul: 2. t. 4. $f$. 4. 5. Lacerta viridis.

Habitat in Europa, Indis.

Variat in aufrali Europa colore viridi; Indica pulchrius colnata. Fennora poftica jubtus linea e punctis calInfis notata, $\because t$ in L. Amleiva.

algira. 16. L. cauda verticillata longiufcula, corpore lineis vtrin: que duabus flauis.

Habltat in Mauritania. E. Brander.

Corpus aix digito longius, Jupre fufcum, fubtus plas eefiens. Squama dorfa!cs carinatae, acutiones. Linea :trinque flaua dorjinn includit at alia strinque abdomen diflinguit a lateribus. 


\section{AMPHIBIA REPTILIA. Lacerta.}

Seps. I7. L. cauda verticillata longa, futura laterali reflexa, fquamis quadratis.

Amoen. acad. I. p. 293. Lacerta cauda verticillata, pedibus fubpentadactylis, fquamis quadrutis. ${ }^{*}$

Habitat in Meridionalibus.

Pedes breues remotiffmi, curforii; Áudomen plamum.

angulata. I8. L. cauda hexagona longa, fquamis carinatis mucronatis.

Habitat in America. Rolander.

Parua dorjo fufco. Squamac omnes, exceptis abdominalibus, mucronatae, valde carinatae. Caput mudum, rugis variis eleuatis: poftice, vbi fquamae colli incipiunt, quafi truncatum et anmexum; jib Gula fiuanicue 2, magnae, rotundatae. Cauda corpore jêsquilongior, bexagona, valde angulata.

* * Cauda tereti imbricata, corpure breniore.

Chamae- I9. L. cauda tereti breui incurua, digitis duobus tribusleon.

que coadunatis.

Amoen. acad. I. p. 290, 5or. Idem.

Muf: $A d . F r$. I. $p .45$. Idem.

Gron. mus. 2.p. $76 . n .50$. Chamaeleon.

Olear. mu. 9.t. $8 \cdot f \cdot 3$. Chamaeleon.

Bellon. itin. l. 2. c. 6o. Chamaeleon.

Besl. muj: t. I2. Chamaeleon.

Valent. muf: l.3.c.3r. Chamaeleon.

Kircber. mus. $275 \cdot t .293 \cdot f \cdot 44$. Chamaeleon.

Ionjt. quadr.t. 79 . Chamaeleon.

Seb. milf. I. t. 82. f. 3. 4. 5. et t. 83. f.5.

Aldr. quadr. 670. Chamaeleon.

Habitat in Africa, Afia.

Anatome Chanaeleontis. Haljelq. iter. 297."

Variat vertice plano et carinato. Intumefcit detumefcitque lente corpus. Oculi tecti: pupilla nuda aurata fulgentiffimi. Ince Jus tardus pedibus oppofite anomalis caidaque jicandente. Lingua lumbriciformis, longiffima, mulcicapa. Colores mutat in calidis: noct $u$ vigil.

Salaman-20. L. cauda tereti breui, digitis muticis, corpore porofo dra. nudo.

Amoen. acad. I. p. I3r. Lacerta cauda tereti breui, pedibus inermibus: palmis tetradactylis, plantis pentadactylis. 


Muf. Ad. Fr. I.p. 45. Lacerta eadem. Matth. diofc. 274. f. 274. Salamandra.

Geji. quadr. 80. Salamandra.

Aldr. quadr. 64I. Salamandra terreftris.

Ionft. quadr. t. $77 \cdot f$. 10.

Olear.mul: t. 8.f. 4 .

Seb. muf.2.t. 12.f. 5 .

Rai. quadr. 173. Salamandra terreftris.

Habitat in Europa.

Corpus nudum absque fquamis, poris pertufum.

Ferebatur antiquitus in igne viuere, fed expertus Bartbolinus; oleum e poris exfudans pro pfilotro babetur.

Gecko. 2I. L. cauda tereti mediocri, digitis fubtus imbricatis, corpore verrucofo.

Amoen. acad. I. p. I33. 292. Lacerta cauda tereti mediocri, pedibus pentadactylis: digitis criftatis imbricatis, corpore verrucofo.

Mul: Ad. Fr. I. p. 46. idem.

Haffelqu. iter. 306. Lacerta Gecko cauda tereti me. diocri, pedibus criftatis fubtus longitudinaliter 12. mellofis.

Gron. mul: 2. p. 78. n. 53. Salamandra.

Seb. mif. I. t. 168. $f \cdot 2-8$.

Bont. iau. 57. Salamandra indica.

Habitat in Indiis, fiequens etiam in domibus.

Pedibus exbalat venenuri in e(culentis (an vrina?) Hasfelq. Manfueta; bomo afylum.

Stincus. 22. L. cauda tereti mediori apice compreffa, digitis muticis marginatis.

Gron.muj: 2.p.76.n. 49. Scincus.

Sib. mil. 2. p. 112. t. 105. f. 3 .

Befl. mus. I. I2, $f$. I.

Olear. muf. 9. t. 8. $t^{\circ}$.

Rai. quadr. 27I. Scincus.

Amnen. acad. I. p. 294.

Haffelqu. att. vpf. 1,50.p. 30.

- - itin. 309. n. 58 .

Habitut in monto/ss Lybiae, Aegypti, Atabiae patreas.

Corpus officinale pro aplorodifiaco.

hifpida. 23. L. cauda tereti mediocri, vertice trinuricato. Mil:: Ad. Fi: I. p. 44. Lacerta eadem. 
Seb. muf. I. t. Iog. $f .6$.

t. 83. f. . 2.

Habitat in America auftrali.

orbicula- 24 . L. cauda tereti breui, trunco fubglobofo fupra muris. ricato.

Seb. mm . I. p. I34. t. 83. f. I. 2. Lacertus orbicularis fpinolus.

Hernand. mex. 327. 328. Lacertus orbicularis.

Rai. quadr. 263. Lacertus orbicularis.

Habitat in Mexico.

vulgaris. 25. L. cauda tereti mediocri, palmis tetradactylis, pedibus muticis.

Faun. fuec. 254. Lacerta pedibus inermibus, manibus tetradactylis, plantis pentadactylis, corpore liuido: linea dorfali fufca duplici.

Rai. quadr. 264. Lacerta vulgaris.

Habitat in Europa, Lavua fub aquis.

aquatica. 26. L. cauda teretiufcula mediocri, pedibus muticis fifis: palmis tetradactylis. Fn. Juec. 257 . Syft.nat.6.p.36.n.

Gron. muf: 2. p. 78.n.52. Salamandra alepidota, cauda teretiufcula.

Gefin. ouip. 3I. Lacertus aquaticus.

Seb. mu:: 2.p. 15. t. I2. $f$. 7 . Salamandra zeylanica. Habitat in Europae aquis dulcibus, ftagnantibus.

Vtrum baec diftinct a jeccies vel larua prioris nondun rite perjpectum babeo:

* * * Cauda tereti imbricata, corpore longiore.

Bafili- 27 . L. cauda tereti longa, pinna dorfali radiata, occipite fcus. criftato.

Seb. muj: I. t. 100. f. I.

Habitat in America auftrali.

Iguana. 28. L. cauda tereti longa, futura dorfali dentata, crifta gulae denticulata.

Amoen. acad. r. p. 123. 287. Lacerta cauda tereti, pedibus pentadactylis, crifta dorfi longitudinali, gulae pendula antice dentata.

Minf: Ad. Fr. I. p. 43. Idem.

Gron.muf. 2.p.82.n. 60. Iguana.

Marcgr. braf. $236 . f .236$. 


Ionf. quadr.t. $77 \cdot f \cdot 5$.

Iacob. mus. t. 4 .

Olear. mus. t. 6.f. I. Yuana.

Bont. iau. 56.t. 56 . Lacerta Leguan.

Marcgr. braf: 236. Senembi f. Iguana.

Nieremb. nat. 271. t. 271 .

Ovied. amer. l. 13. c. 3.

Red. exper. IOO. t. IOI. Iguane.

Worm. mus. 313 .

Sloan. iam.2. p. 333 .

Rai. quadr. 265. Lacertus Senembi et Iguana;

Seb. minf: I. $t$. 95. f. I. 2.

$$
\text { 96. } f .4 \text {. }
$$

98. $f$. $\mathrm{r}$.

Clue. exot. II6. Yuana.

Catesb. car.2. p. 64.t. 64 ?

Habitat in Indiis.

Capitur linea laqueari. Caro onmium Sapidifima, Sipliliticis noxia.

Calotes. 29. L. cauda tereti longa, dorfo antice capiteque poftice dentato.

Anoen. acad. I. p. 289.

Muf. Ad. Fr. I. p. 44.

Seb. $9 n u l:$ I. t. $95 \cdot f: 3,4$.

t. $93 \cdot f .2$.

Habitat in Afia: Zeylona.

Corpus caeruleum finamis acutis, fubtus friatum. Spinae dorfi lamellatae.

Agama. 30. L. cauda tereti longa, collo fupra capiteque poftice aculeato.

Amoen. acad. I. p. 288.

Muf. Ad. Fir. r. p. 44.

Seb. muf. I. t. 107 . f. 1. 2.3.

Habitat in America.

Color corporis pallidus. Abdomen minime friatums.

Vmbra. 31. L. cauda tereti longa, nucha fubcriflata, occipite cal. loio, dorfo ftriato.

Halitat in Meridionalibus.

Plica profinda Jib gula. Color curporis usbulofus. 
Plica. 32. L. cauda tereti longa, occipite callofo: palpebris fupra excoriatis, collo lateribus verrucofo fubtus plicato.

Habitat in Indiis.

Corpus praeter caudam vix digito maius, in meo Specimine, tectum vndique fquamis conicis (velut Chagrin). Occiput callojun et Supercilia fibcrenata, fupra excoriata cicatrice menbranacea transuer fe in 3 partes fulco diftincta. Pone aures ad latera capitis et colli verucae 2, muricatae. Collum jibtus duplici plica. Dorfi fitura maioribus fquamis antice quafi crentata. Ruga cleuata a collo fupra bracbia excurvit utrinque et in medio trunci deflectitur. Cauda teres, tect a fquamis minuti/fmis; vix manifefte verticillata, corpore duplo longior. Digiti longi, Jibtus Jquanis acutioribus fcabri; vngues compreffe.

marmo- 33. L. cauda tereti longa, gula fubcriftata antice dentata, rata.

\author{
dorfo laevi.
}

Annoen. acad. I. p. I29. 288 .

Mul: Ad. Fir. I. p. 43 .

Seb. mul: 2. t. $76 . f .4$.

Habitat in Hifpania.

Corpus compreffun; Cauda ftriata.

bullaris، 34. L. cauda tereti longa, vefica gulari. :

Catesb. car. 2. t. 66. Lacerta viridis iamaicenfis.

Habitat in Iamaica.

Parua, corpore viridi; Jub gula globus ruber veficularis retractilis.

ftrumofa. 35. L. cauda tereti longa, pectore gibbo protenfo.

Seb. mus: 2. t. 20. f. 4. Salamandra mexicana ftru. mofa.

Habitat in America aufroli.

Pectus f: Sternum antice prominet in mucronem obtus funn.

- Tegui- 36. L. cauda tereti longa, futura laterali plicata.

xin. Anvon. acad. I. p. I28. Lacerta cauda tereti longa, pedibus pentadactylis, crifta nulla, hypochondriis pli cat:s.

Mul: Ad. Fr. I. p. 45 .

Seb. mif. I. $t .96 . f . \mathrm{I}$.

Habitat in Indiis.

Collum fubtus triplici plica. 


2urata. 37. L. cauda tereti longiufcula, fquamis rotundatis glabris, lateribus fubfufcis.

Annon. acad. I. p. 294. Lacerta cauda tereti, pedibus pentadactylis, fiuamis rotundatis laeuifimis fubgrifeis: lateralibus fubfulcis.

Muf: Ad. Fr. I. p. 46. Lacerta barbara.

Gron. mui. 2. p. $75 \cdot$ n. 48 . Scincus?

Seb. mul. 1. t. $89 \cdot f .3$.

Aldr. quadr. 660. Lacertus cyprius fcincoides.

Habitat in Ierfea Anglorum, Cyproz

Viua colore auri pulchervime nitet.

Corpus teres quafi pingue. Aures concauae.

punctata. 38. L. cauda tereti longa, dorfo lineis duabus flauis: punEtis nigris interfperfis.

Mirf: Ad. Fr. p. 46. Lacerta eadem.

Sib. mul: 2. t. 2. $f$. 9 .

Habitat in Afia.

Lincae 2. flauefentes includant dorfum et a lateribus eandem diftingumut.

lemni- 39. L. cauda tereti longa, dorfo lineis octo friatis.

fcata. Mul: Ad. Fr. I. p. 47. Lacerta eadem.

Scb. mus. 1. $t$. $92 . f^{\prime} \cdot 4$.

Habitat in Guinea.

Multum affinis L. agili.

fafciata. 40. L. cauda tereti longiufcula, dorfo brunneo lineis quinque flauefcentibus.

Catesb. car. 2. t. 67. Lacerta cauda caerulea.

Pet. gaz. I. t. I. $f$. I.

Habitat in Carolina.

lineata. 4I. L. cauda tereti longa, palmis tetradåtylis, corpore lineis quatuor flauis.

Muf. Ad. Fr. 1. p. 46. Lacerta cauda tereti longa, pedibus fubunguiculatis firis, palmis tetradactylis, plantis pentadactylis.

Seb. muf:. 2. t. 4r. f. 6. Lacerta zeilonica minor lemnifcata.

Habitat in Zeilons.

Chalci- 42. L. cauda tereti longa, pedibus tridactylis. des. Syjt. nat. 36.n. 7. Lacerta eadem. 
Colusnn. ecphr. 1. p. 35. t. XXXVI. Seps, Lacerta Chaicidica f. Chalcides.

Aldr. quadr. 638. Lacerta chalcidica.

Habitat in Europa aufrali, Africa.

Media inter Lacertas et Angues, fed muribus inftructa.

anguina. 43. L.: cauda extremo rigidula, corpore ftriato, pedibus pinniformibus. $\dagger$

Seb. muf. 2. $t .68 \cdot f \cdot 7 \cdot 8$. Vermis ferpentiformis ex Africa.

Habitat in Cap. B. Spei lutojis, mibi non vifa.

106. R A N A. Corpus tetrapodum, nudum, ecaudatum.

Pipa. I. R. digitis anticis muticis quadridentatis, pofticis vnguiculatis.

Muf. Ad. Fr. I. p. 49. Rana eadem.

Gron. mul.2.p. $84 \cdot n \cdot 64$.

Scb. mml:. I. p. I21. t. 77. f. I-4. Bufo I. Pipa americana.

Vincent.pip. 1726. t. 62. Bufo aquaticus furinamenfis. Bradl. nat. I. t. 22. f. I. Rana furinamenfis.

Vallifn. nat. I. t. 4I. f. 6 .

Habitat Surinami.

Pullos e dor.jo nidulantes excludit.

Bufo. 2. R. corpore ventricofo verrucofo lurido fufcoque.

$F_{n}$. Juec. 253. Rana palmis tetradactylis fiffis, plantis hexadactylis palmatis: pollice breuiore.

It. oel. 142 .

Gefn. pifc. 807. Rubeta f. Phrynum.

Ionft. quadr.

Bradl. nat. t. $2 \mathrm{I} . f .2$.

Rai. quadr. 252. Bufo R. Rubeta.

Habitat in Europae nemorofis ruáeratis vmbrofis, imprimis Vcraniae.

Deuoratur a Falcone Buteone . Gefn. pifc. 807.

Vermicae lactefcentes venenatae infulo, tactu, balitu. Delectatur Cotula, Actaea, Stachyde foetidis. Viuipara, mare, vt fertur, obftetricante. Infecta in fauces fafcino reuocat; nocturna, fodiens.

3. R.

Palmae in plerisque Ranae Speciebus fiffae tetradactylae, Plantaeque pentadactylae palmatae, rarius vero obfcure hexadactylae. 


Rubeta. 3. R. corpore verrucofo, ano obtufo fubtus punctato. Syft. nat. 37. n. 5. Rana palmis tetradactylis fifis, plantis pentadactylis fubpalmatis, ano fubtus punEtato.

It. Wgoth. $26 \mathrm{I}$.

Habitat in Europa.

gribbofa. 4. R. corpore ouato-conuexo: vitta longitudinali cinereodentata, pedibus fiffis.

Anven. acad. I. p. 286. Rana palmis tetradactylis fiftis, plantis hexadakylis fiflis, pollice latiufculo breuiffimo.

Mul: Ast. Fr. r. p. 4S. Rana eadem.

Habitat in Exteris regionibus.

variega- 5. R. corpore verrucofo, abdomine albo nigro-maculato,

t2. plica gulari.

Habitat in Exteris regionibus.

Bufoni finilis jed parua, nigra, fupra vndique punctis exajperata, fubtus albo nigroque varia. Pedes mutici: Palmis tetradactylis fifis; Plantis pentadactylis palmatis. Ruga transuerga fub collo.

ventrico- $6 . R$. ore femiouato, iugulo prominulo.

f. Mis. Ad. Fr. I. p. 48. Rana eadem. Habitat in Indiis.

marina. 7. R. fcapulis gibbofis, clunibus nodofis.

Seb. mul. I. t. 76. $f$. I. Rana marina maxima.

Habitat in America.

Palmae tetradactylae fipac; Plantae pentadactylac fub. fifjac.

typhonia. 8. R. auricularibus lobis ouatis.

Habitat in America, clanitans nocle fono cornicis tetra lucente. Rolander.

Dorfum rugis quatuor longitudinalibus, puntis cleuntis, maculisque nigris. Pedes nutici. Palmae tetradadylae fifae: Plantae pentadactylae palmatae : digitis anguflis: Jecundo longifino, absque vnguibus orbiculutis.

ocellata. 9. R. auribus ocellatis, pedibus muticis.

Broron. iam. 466.t. 4I. f. 4. Rana maxima compreffa mifcella.

O 4

Habi-

Jenes Ranarum an verrucae pollicis palmarum? Penis enim verque in Tefindine ad lxmbos femorkm, in Serpentibus ad anxm: Ona Ranarwne plane nuda $\int u n t$. 
Habitat in America.

Ad aures macula ocellaris vtrinque. Palmae tetrada. ifylae fiffae, Plantae pentadactylae, Jubpalmatae.

cornuta, IO. R. palpebris conicis.

Mir. Ad. Fr. I. p. 48. Rana eadem.

Seb. muf. I. $t .72$. $f .1,2$. Bufo cornutus $\mathrm{f}$. fpinofus virginianus.

Habitat in Virginia, adfpectu borrenda.

margina- II. R. lateribus marginatis, corpore laeui, plantis fiffis.

ta. Muf: Ad. Fr.p. 47. Rana lateribus marginatis. * Habitat in Indiis.

paradoxa, 12. R. femoribus poftice oblique ftriatis. Muf. Ad. Fr. 2. p...

Syft. nat. 36. n. 2. Lacerta cauda ancipiti, palmis tetradactylis fifis, plantis pentadactylis palmatis, abdo. mine ventricofo.

Muf. Ad. Fr. I. p. 49. Rana pifcis.

Seb. muj: I. t. 78,f. omnes.

Merian. Jurin. 7I. t. 7 I.

Habitat Surinami.

tempora- 13, R. dorfo planiufculo fubangulato.

ria. Laun. fuec. 250. Rana palmis tetradactylis fiffis, plan. tis pentada\&tylis palmatis: pollice longiore.

It. oel. 154. Rana temporaria.

Gefin. ouip. 46. Rana aquatica innoxia.

Aldr. ouip. 89. Rana.

Ionft. quadr. t. $75 . f .5,6,7,8$.

Rai. quadr. 247. Rana aquatica.

Bradl. natur, t. 2I. f. I.

Habitat in Europa.

Vere aquatica, aeftate terreftris, victitat Culicibus. Deuoratur ab Anatibus, Ardeis. Corde licet exento falit.

efculen: I4. R. corpore angulato, dorío transuerfe gibbo, abdomi-

ta. ne marginato.

Roef. ran. 5I. t. I3. Rana viridis aquatica.

Habitat in Europae fontibus.

Viridis lineis 3. flauis: intermedia ab ore ad anum ducta. Dorfum transucr.j gibbofo-diffiractum. Pedes poftici palnati. Mas auribus globojo-inflatis. Cantu vejpertino frequentiors praedicit pluniam. 


Hyla. I5. R. dorfo angulato transuerfe gibbo, abdomine fafcia replicata inguinali intercepto.

Gefn. pifc. 809. Rana gibbofa 4, 5 .

Habitat in Europa.

Sonus campanaritn boatun e.longinquo innitatur.

arborea, I6. R. corpore laeui : fubtus punctis contiguis tuberculato, pedibus fiffis; vnguibus orbiculato-dilatatis.

Amocn. acad. I. p. 135. Rana pedibus fiffis; vnguibus fubrotundis, corpore laeui: poftice anguftato.

Murf. Ad. Fr. I. p. 47. Rana eadem.

Gron. muf. 2. p. 84. n.63. Rana etc.

Seb. mut. I. t. $73 \cdot f \cdot 3$. Rana brafilienfis gracilis.

Seb. mulf. 2. t. 70. f. 5. Rana americana rubra.

Gefir. pifc. 809. Ranunculus vitidis.

B. Amoen. acad. I. $p .=85$. Rana pedibus fiffis, palmis tetradactylis, plantis pentadactylis: geniculis fubtus tuberofis.

Habitat fub foliis arbortun Europae, Americae, Mufcas in fauces reuocans.

boans. I7. R. corpore laeui : fubtus punktis contiguis, pedibus palmatis: plantis pentadactylis, palmis tetradactylis, vnguibus orbiculato - dilatatis.

Amoen. acad. I. p. 255. Rana palmis tetradactylis, plantis pentadactylis palmatis, digitorum apicibus fubrotundis.

Muf. Acl. Fr. I. p. 47. Rana eadem.

Seb. mul. I. t. .7. f. 3. 4. Rana furinamenfis.

Habitat in America.

Simillima R. arboreae, fed pedes omnes palmati et corpus album magnum, maculis etian lakteis; modo baec Jüficiant pro jpecie diffingruenda.

O 5 


\section{SERPENTES.}

Os refpirans.

Pedes Pinnaeue nullae.

107. CRO TALVS. Scuta abdominalia.

Scuta Squamaeque fubcaudales.

Crepitaculum terminale caudae.

Scutis: Scutellis:

192. horridus. $167-23: 2$. Mus. Ad. Fr. I. p. 39.

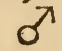

Bradl, natur. t. 9. $f$. I.

Scb. Muf. 2. t. 95 . f. I.

Habitat in America.

Venenatiffinus; antidotun Senega: a Sue confunitur; Aues Sciurosque ex arboribus in fauces renocat.

195. Dryinas. 165-30. Anzoen. acad. I. p. 297.

$\begin{array}{ll}\text { o } & \text { Habitat in America. } \\ & \text { Exalbidus maculis flauefcentibus paucis. }\end{array}$

196. Durifus. I72-2I : 3. Annocn. acad. I. p. 500.

o

Kalm. act. Stockb. 1752. p. 310. et 1753 . p. 52, 185.

Gron. muf: 2. p. 70. n. 45. Crotalophorus I74-22: 3 ?

Habitat in America.

Albo flatroque varius, maculis rbombeis atgivis difco albis.

108. BOA. Scuta abdominalia.

Scuta fubcaudalia (absque crepitaculo.)

276. Scytale. 250-26. Gr.on. mu! 2. p.55. n. IO.

Scheuch. facr. t. $737 \cdot f$. I.

Habitat in America.

Color ex albo nigroque vndulatus. Squamae capitis maiores.

180. 


280. canina. $203-77$. Muf. Ad. Fr. r. p. 39. t.3.

Scb. mul:2.t. 96. f.2.

$$
\text { t. SI. f. I. }
$$

Habitat in Americae arboribus.

Viridis, fafciis albis interruptis.

Adoratur ab Americanis. Rolander.

299. Hipnale. 179-I20. Seb. mut. 2. t. 34. f. 2.

Habitat in Afia.

Grijeo-flauefcente varius.

300. Conftri- 240-60. Amoen. acad. r. p. 497.t. I7. f.3.

Btor.

Mul: Ad. Fr. I. p. 38 .

Gron.mul: 2. p.69.n.43. Cenchris $248 \cdot 60$.

Seb. $m$ muf:2.t.98. f: I.

t. 99. f. I, 2.

t. IOO. $f$. $\mathbf{r}$.

t. 10I. $f$. I.

t. I04. $f . \mathrm{I}$.

I. $t .53 \cdot f . \mathrm{I}$.

t. $36 . f .5$.

Habitat in Indiis.

t. $62 . f .2, \mathrm{r}$.

519. murina. 254-65. Gron.muf. 2. p. 70. 12.44. Coluber $254-69$. Seb. mus. 2. t. 29. $f$. $\mathbf{~}$.

Habitat in America.

Rufus maculis Jupra rotundatis.

322. Cenchria. $265-57$.

IIabitat Surinami.

Flanejcens ocellis albidis iride grifea.

345. Orophias. 28I-64. Muf. De Geer.

Habitat . .

Facic Conftrictoris, fed fufcus.

375. Enydris. 270-105. Muf. Dc Geer:

Habitat in America.

Variegatus colore grifeo. Dentes inferiores longi.

41S. Hortula- 290-128. Muf. Ad. Fr. I. p. 37.

na.

Scb. mutl: $2 . t .84 . f .1$.

t. $74 . f . \mathrm{I}$. 
Habitat in America.

Pallida maculis lindis cuneiformibus. Caput areolis luteis borti inftar.

109. COLVBER. Scuta abdominalia. Squamae fubcaudales.

I40. Vipera.
đ

$$
\begin{aligned}
& \text { II8-22. Hajfelq. Act. VpS. } 1750 \cdot p \cdot 24 \text {. } \\
& \text { itin. } 3 \mathbf{1 4} \cdot n .60 . \\
& \text { Habitat in Aegypto. } \\
& \text { Breniffima, capite gibbo, fquamis minutis. } \\
& \text { Officinalis baec Vipera eft aegyptionum, nec } \\
& \text { Berus. }
\end{aligned}
$$

153. Atropos. I3I-22. Muf. Ad. Fr. I. p.22.t. I3.f. I. Habitat in America.

Canus, ocellis fufcis iride alba.

160. Leberis. Iro-50.

o

I6I. Lutrix.
Habitat in Canada. Kalm.

Fafciae lineares nigrae.

Habitat in Indiis.

Dorfum et Abdomen flaua; Latera caerulejcentia.

162. Calama- 140-22. Mirf. Ad. Fr. I. p.23. t. 6.f. 3 . rius.

Habitat in America.

Liuidus fafciis punctisque linearibus fufcis; jubtus fufco tefjellatus.

170. Confri- 130-40. etor.

Habitnt in Canada. Kalm. Maxillae apex finns triqueter. Adoritur bomines circum pedes fefe conuoluens, fed innocuus.

I74. Ammo- I42-32. Annoen. acad. I. p. 506. n. 25. ơ dytes. Bellon. itin. 203: Druinus. Aldr. Serp. 169. Ammodytes. 


Habitat in Oriente.

Najics terminatus verruca erecta.

175. Ceraftes. 150-25. Haffelqu. ACt. Vpf: 1750. p.27. iter. 315. \%. 61. Coluber cor.

Bellon. itin. 203. nutus.

Habitat in Oriente.

Squanae capitis omnes paruae rotundatar. Dens mollis exit e falpebra Juperiore.

177. plicatilis." I3I-46. Amven. acad. I. p. 301. n. 26.

Muf. Ad. Fr. I. p. 23 .

Seb. mul: I. t. $57 \cdot f \cdot 5$.

Habitat in Ternataeis.

Liuidus lativibus fufcis; fubtus punctis triplici urdine fufcis.

178. Domicel- IIS-60. Amoen. acad. I. p. II7.n. 5.

la. Seb.mil.2.t.54.f. I.

Habitut in Alia.

Albus fic ciis nigricantibus, fubtus concurrentibus.

179. Alidras. I2I-58. Muf. De Gerr. Habit at in Indiis.

Albus totus.

180. buccatus. 107--72. Muf. Ad. Fr. p. 29.t. 19. f. 3 . Habitat in Indiis.

Fulcus fajciis albis. Caput album: fufcis punctis 2 verticis et triangulo Jispra nas o? res.

181. angulatus.
120-60. Muf. Ad. Fv. I. p. 23.t. 15. f. 1.

Amoen. acad. I. p. I19. n. 7 .

Sil. mil: 2. t. 12. f. 3 .

Habitat in Alia.

Grifeo-filfus fafciis nigris.

183. Berus. 146-39. Finnn. Jivec. 260.

Ansoen. acad. I. p. I13. n. 1.

Alat: Jivp. 115, 116 .

Habitat in Luropa. 
184. Cherfea. 150-34. Faun. Juec. 26r.

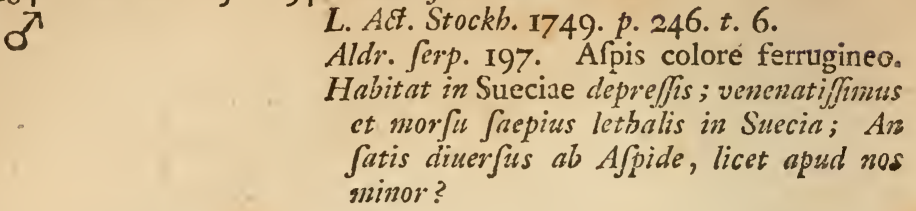

189. caeruleus. 165-24. Amoen. acad. I. p.303. $2.3^{\mathrm{r}}$.

Seb. muJ. 2. t. 13. $f \cdot 3$.

Habitat in America.

Cacrulefcens fquamis altero latere albis: fubtus albus.

190. albus. I70-20. Mu؟. Ad. Fr. I. p.24.t.14.f.2.

Habitat in Indiis.

Albus immaculatus.

192. Afpis. 146-46. Gallis Afpice.

$\sigma^{\lambda}$

Habitat in Gallia.

Rufus maculis dorfulibus fufcis alternis cono. fuentibus in vittam.

Similis Cberfeae, fed maior.

193. Typhlus. 140-53. Muf. De Geer.

Habitat in Indiis.

Caerulefcens.

20r. Lebeti- I55-46. Hafjel quift.

$\sigma^{7}$ nus.

Habitat in Oriente.

Subnebulatus, fubtus punctis fufcis.

202. melano- 140-62. Muf. Ad. Fr. 1. p. 24. t. I5. f. 2. cephalus.

Habitat in America.

Fufcus glaberrimus capite atro.

204. Cobella. 150-54. Amoen. acad. I. p. I17. n. I4.

$$
\begin{aligned}
& \text { p. } 302 . n .28 . \\
& \text { p. } 496 . n .14 \text {. }
\end{aligned}
$$

Gron.mur. 2, p. 65.n. 32 .

Seb. muj: 2. $t$. 2. $f .6$.

Habitat in America frequentiffimus.

Cine.

Colubri Squamae caudales numerantur longitudinales, $\int$. per paria, quamwis alternae, сиm duae fefe babant, vt vnicum $\int$ cutum $\int u b$ abdomine. 




\section{AMPHIBIA SERPENTES. Coluber.}

Cinereus adfperfus lineolis oblinuis albis. $M a$ cula obliqua plumbea purze fingulos oculos.

207. Reginae. I37-70. Muf. Ad. Fr. p. 24. t. I3. f. 3 .

Habitat in Indiis.

Fufius abdomine albo nigroque vario.

212. feuerus. o

$$
\text { 170-42. Mus. Ad. Fr. I. p. 25.t. S.f. I. }
$$

Seb. muf. 2. t. 54. f. 4 .

Habitat in Afia.

Cinereus fafciis albis. Fafcia cinerea inter oculos et pone nares.

216. Aurora. 179-37. Muf: Ad. Fr. p. 25. t. I9. $f$. I.

Seb. mul. 2. t.78. f. $\hat{\jmath}$.

- Hobitut in America.

Lividus dorfo flauso.

217. Sipedon. I44 $\rightarrow$ 73. Kalm.

Habitat in America Septentrionali.

Fiufous.

218. maurus. $152-66$.

Haủitat Algiriae. E. Prander.

Corpus jupra fufcum lineis dorfalibus duabus; jabtus atrum; a lineis dorjalibus ad abdomen vtrinque fafciae plures nigras.

219. folatus. I43-76. Muf. Ad. Fr. I. p. 26. t.22. f. I.

Seb. mul. 2. t. 9. f. 1.

Habitat in America.

Grifeus vittis 2 albis.

Scuta vtrinque puncio nigro.

220. vittatus. 142-78. Amoen. acad. I. p. 30. 17. 27.

Muf. Ad. Fr. p. 26. t. 18.f. 2.

Gron. mui 2. p. 65. n. 3i. Coluber 155-62i

Scb. mus. 2. t. $45 \cdot f \cdot 5$.

t. $60 . f: 2,3$.

Halitat in America.

Scuta margine fujca.

Vittu alba dentiata jiub cauda.

22 .

Serpentes noftrates bylernant, et primo vere exexntes вxкиias fo fentatav exน?nt. 
221. miliaris. 162-59. Muf. Ad. Fr: p. 27.

\section{Habitat in Indiis.}

Fujcus: fquamis macula alba. Subtus albus.

227. rhombea- 157-70. Mur. Ad. Fr. p. 27. t. 24. f. 2.

tus.

Habitat in Indiis.

Cacrulefcens maculis nigris medio caeruleis ribombeis.

229. cyaneus. II9-IIO. Amoen. acad. I. P. 493.n. Io.

Seb. mul: 2. t. $43 \cdot f \cdot 2$.

Habitat in America.

Saturate caeruleus, babitu Abetullae; fubtus virens.

230. Natrix. 170-60. Faun. Juec. 259.

It. gotl. 146 .

Amocn. scad. I. p. II6. n. 3 .

Gron.mul: 2.p. 63. $n .27$.

Habitat in Europa; in finetis pariens.

Niger nilacula alba vitrinque ad collum.

233. Aefcula- 190-43. Amoen. acad. I. p. 497.n. 15.

pii.

Minf. Ad. Fi. I. p. 29. t. II. f. 2.

Gron. muf. 2. p. 59. n2. I8.

Seb. mul: 2. $t$. 18. $f .4$.

Habitat in Indiis.

Faficiae albae nigraeque, bae linea annulo. ue albo dimidiatae.

234. agilis. I84-50. Amoen. acad. I. p. 304. n. 33 .

Muf. Ad. Fr. I. p. 27.t.2I. f. 2.

Habitat in Indiis.

Fafciae fufcae albaeque.

235. lacteus. 203-32. Muf. Ad. Fr. I. p. 28.t. I8.f. I. Habitat in Indiis.

Albus maculis geminatis atris. Vertex ater linea longitudinali alba.

244. aulicus. 184-60. Muf. Ad. Fr. r. p. 29. t. 12. f. 2.

Seb. mul: I. $t$. 9I. $f$. 5 .

Habitat in America.

Gria

Serpentes Praedam, collo dxplo craffiorem, fatpe ingurgitant, ob maxillas dilatabiles, nec articulatas. 




\section{A MPHIBIA SERPENTES. Coluber.}

Grifeus fufciis albis latere bifurcatis. Tertex albus.

246. monilis. I64-82. Muf: De Cieer.

Habitat in America.

Corpus anmulatum; monile punctis 3 albis a tergo.

25I. pallidus. I5p-96. Amoen. acad. I. p. 494.n. II. Mut: Ad. lir. I. p. 3I. t. 7. f:2.

Habitat in Indiis.

Pallidus maculis grifois vagis puntisque fufisis. Lineolae binae intermutae laterales migricantes.

252. lineatus. 169-84.Muf. Ad. Fr. I. p. 30. t. I2. f. r.

Seb. muf. 2. t. 12. f: 3.

$$
\text { t. 20. } f: I_{0}
$$

\section{Habitat in Afia.}

Vaja Caerulejcens vitlis linearibus 4 fufcis.

253. Naia.

o

193-60. Misf. Ad. Fr.p. 30.t.2I. f. x.

Sib. Imis. 2. t. 90. f. I, 2 .

$t .85 \cdot f$. I.

t. 89. f. I, 2, 3, 4.

t. $97 . f .1,2,3,4$.

t. $94 . f . \mathrm{I}$.

I. $t .44 . \because .1$.

Kacmipf. amoen. $565 .+5 \cdot 567$.

Anocn. acad. I. p. 305.

Habitat in India orientali.

Colli latera dilatata in membranam Supra pictan perfpicillis albis.

Onnium vonenatiffmus; antidutun Oploiorrhisa; ab Ichneunnone occiditur.

254. Padera. 198-56. Mirf: Ad. Ir. 2. p...

Hubitat in Indiis.

Macularum fulcarmm multa paria fecun dum dorfirm connexa lineoln; totidemque fimplice's maculae ad latera.

258. canus. $\quad$ ISS $\rightarrow$ O . Mul: Al. Fy. I. p. 31. t. I1. $f$. I. Habitat in Indiis.

Canus

Telo venenato reflexili armatos, figno on notatos, obferna. 
Canus falciis fubfufios; ad latera pumata 2 nillea.

260. fibilans. i60-100. Amoen. acad. I. p. 302. 2.30 .

Seb. mmul: 2. t. 52. f. 4 .

t. $56 . f .4$.

Habitat in Afia.

$$
\text { t. } 107 \cdot f \cdot 4 \text {. }
$$

Cacralejcens vittis nigris, fubtus albus.

261. laticauda-220-42. Muf. Ad: Fr. r. p. 31. t. 16.f. I. tus.

Habitat in Indiis.

Cinereus fafciis fucis.

Cauda obtufa compreffo - anceps.

262. Sirtalis. 150-Ir4. Kalm.

Habitat in Canada.

Vittas tres vividi-caerulejcentes in corpore fujco tèni ftriato.

263. atrox. I96-67. Amoen. acad. x. p. 305. n. 35.

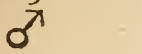

Mus:: Ad. Fr. 1. p. 33.t. 22. f. 2 .

Seb. m11tf. I. t. $43 \cdot t \cdot 5$.

Habitat in Afia.

Canus Jquamis carinatis.

Caput depreffun, compreffun, angulatume jquamis minimis.

265. Sibon. 180-85. Annoen. acad. 1. p. 304.n. 32.

Seb. mule. 1. $t$. $14 . f \cdot 4$.

Habitat in Africa.

Finfó-ferrugineus albo adjperfis; fubtus albus maculis fulcis.

265. nebula- 185-81. Muf. Ad. Fr. p. 32. t.24..f. I.

tus.

Catesl. car. 2. p. 42.t. 42.?

Habitat in America.

Fulco cinereoque nebulatus; fubtus albo fuf:coque varius; adfcendit tibias et firingit.

266. fufcus. I49-II7 Muf. Ad. Fr. I. p. 32.t. I7. $f$. I.

S: 6. mus: 2.t. 54. $f .2$.

t. 7 I. f. I.

t. $72 . f .1$.

t. $87 . f . \mathrm{r}$.

t. $9 \mathrm{I} . f . \mathrm{I}$. 


Habitat in Afra:

Cinereo-fujcus, babitu Abretullae. Pone oculos mucula oblonga fufca.

267. Saturni- 147-120. Muf. Ad. Fr. I. p. 32.t. 9.f. I. nus.

Hauitat in Indiis.

Liuiclus cinereo-nebulofus. Oculi magni.

270. candidus. 220-50. Muf. Ad. Fr. 1. p. 33.t. 7.f. I.

Habitat in Indiis.

Albicans fafciis fincis.

271. niueus. 209-62. Muf. De Geer.

o

Sib. mul. 2. t. I5. f. I.

Habitat in Africa.

Albus immaculatus.

272. fcaber. 228-44. Muf. Ad. Fr. I. p. 36. t. Iof. I.

Habitat in Indiis.

Fufco nigroque nebulatus, fquamis carina. tis. Vertex macula nigra poftice bifida.

273. carinatus. 157-II5. Muf. Ad. Fr. p. 31.

Habitat in Indiis.

Plumbeus Jquanis margine pallidis. Subtus albus. Dorfun carinatum.

275. coralli. r93-82. Muf. Ad. Fr. I. p. 33.

$\delta$ nus. Seb. muf: 2. t. 17.f. I.

Habitat in Afia.

Glaucus vittis tribus fincis, fquarris dijtane tibus; Jibtus pallidus punctis canis.

276. ouiuorus. $203-73$. $K a \ln$.

Pif: braf. 279. Guinpuaguara.
Habitat in America.

279. exoletus. 147-132. Muf. Ad. Fr. I. p. 34. t. IO.f. 2.

Habitat in Indiis.

Cinereo-caerulejcens, babitu Alsaetullae.

281. Situla. $236-45$.

Habitat in Aegypto. Haßelquift.

Grijius vittis duabus nigris.

P 2

Color in Serpentibus plurimum variat, ve nunquam ex colope ipfis fidendwm. 


\section{AMPHIBIA SERPENTES. Coluber.}

282. Trifcalis. $195-86$.

Habitat in Indiis.

Corpus glaucunn. Lineolae longitudinales dorfales tres finfae ad nucban coniunciae, quarum medius fupra amun definit; infitper in fingulo latere linea fiufia cum prioribus duabus excurrens ad apicem calldae. Cauda $\frac{\mathrm{I}}{5}$.

285. lemnifca.250-35. Amoen. acad. r. p. II\&. n. 6. tus.

p. $493 \cdot n .9$.

Muf. Ad. Fr. I. p. 34. t. I4.f. I.

Sel. mulf. r. t. Iо. fic vit.

Halitat in Afia.

$$
\text { 2.t. } 76 . f \cdot 3 \text {. }
$$

Faficiae albae nigraeque, bae anmulis duo bus albis facepe intervuptac. Corpus glaberrinnunn.

286. annulatus. 190-96. Ankoen. acad. I. p. I20. n. 9.

$$
\text { p. } 305.17 .34 \text {. }
$$

Muf. Ad. Fr. p. 34. t. 8.f: 2.

Seb. maf: 2. t. 33. f. 2 .

Habitat in America.

Albus maculis alternis fufcis rotundis, pase finn conflucentibus.

287. Dipfas. 152-135. Amoen. acad. I. p. 302. ñ. $\approx 9$.

Grow. muf.2. p. 64. n. 30 .

Seb. mus:: 2. t. $24 . f .3$.

Habitat in America.

Caeruleficens fquanis margine albidis. Catlda fubtus fiutura caerulefcente.

290. Pelias. I87-103. Muf. De Geer.

Habitat in Indiis.

Fufcus pone oculos et verticem, reliqua nigro duplicata. Abdonen viride, vtrinque linea flawa.

293. Tyria. 210-83.

Habitat in Aegypto. Haffelquift. Albidus triplici ordine longitudinali macularum rbombearum fuffcarum. 


297. iugularis. $195-102$.

Habitat in Aegypto. Hapelquift.

Niger iugulo jinguinulcnto.

299. Petola. 209-90. Amoen. acad. r. p. 306.n. 36.

P. IIG. $n . S$.

1. 495.013.

Gron. mu! $2 \cdot p \cdot 57 \cdot n \cdot \mathrm{I}_{3}$.

Seb. mul: I. t. 54. f. 4 .

Habitat in Africa.

Plumbius fafciis teflaceis.

307. Molurus. 24S-59. Mur. De Geer.

Habitat ine Indiis.

Simillimus Bone, fed fouta et fquamae capitis maiores, vt in Culubris.

513. Ahactulla. 163-150. Amocn. acad. p. 115. n. 2 .

p. 495. n. I2.

Muf.Ad. Fr. I. p. 35.t. 22. f. 3 .

Gron. mul. 2. p. 6r. n. 24 .

Seb. muf. 2. t. 82. f. I.

2. $t$. $12 . f .3$.

- Bradl. natur. t. 9.f. 2.

Habitat in Afia, America.

Viridi-aureus funamis apice nigris; Fafcia rigra trans oculos.

3'4. petolarius.212-102. Muf. Ad. Fr. I. p. 35. t. 9. f. 2 .

Habitat in Indiis.

Fujcus fafciis alois; fubtus pallidus.

316. Haie.

207-109. Haffilqu. iter. 317. 11. 62. Coluber feutis abd. 206 , fiquam. caudal. 60.

Habitat in Aegynto inferiore.

Maximus, ater fafciis obliquis et fquamis dimidiato albis.

323. filiformis. 165-15s. Mul. All. Fv: p. 36. t. 17.f. .

Habitat in Indiis.

Niger, anguftiflimus, Jibtus albus. Caput corpore crajjius.

325. pullatus. 217-108. Annoln. acad. I. p. 300.n. 25. Mul: Ad. Fi. r. p. 35.t. 20. f. 3 . 
Gron.muf.2.p.56.n. I2. Coluber. 215-104. Sel. mall. 2. t. 20.f. 1 .

Habitat in Alia.

Fafciae atrae pundtis albis. Tempora niuea maculis atris.

326. Hippo- 232-94. Muf. Ad. Fr. 1. p.36. t. 16.f. 2. crepis.

tiabitat in America.

Lividus macuís fufcis. Fafcia fufca inter oculos ét arcuata occipitis.

328. mineruse. 238-90. Mus. Ad. Fr. I. $p \cdot 36$.

Hzbitat in Indiis.

Glaucus vitta dorjali fufca. Vittae capitis 3 fuficae.

337. cinereus. 200-I37. Muf. Ad. Fr. I. p. 37.

Habitat in Indiis.

Cinereus abdomine albo angulato. Caudae fquanae margine ferrugineae.

339. viridiff- 217-122. Muf. Ad. Fr. 2. p...

mus. Hitbitat Surinami.

Viridiffmus, foutis abdominis medio dila. tatis.

340. mucofus. 200-140. Muf. Ad. Fr. I. p. 37.t. 23. f. i. Habitat in Indiis.

Caput caerulejcens.

344. Cenchoa. 220-124. Amoen. acad. I. p. 306. n. 37.

Seb. minf. 2. t. 16. f.2, 3 .

Habitat in America.

Fulcus maculis pallidis fajciis niueis. Caput fubglobojum.

359. mycteri- I92-167. Muf. Ad. Fr. I. p. 2s. t. 5.f. I.

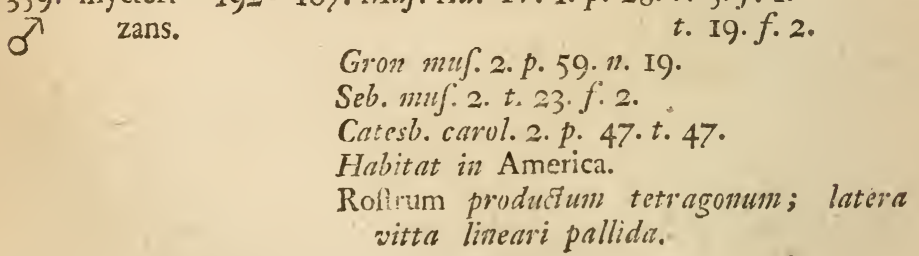




385. caerule- 215-170. Muf. Ad. Fr. I. p. 37.t. I0.f. 2.

fcens. Habitat in Indiis.

Cacrulejcens.

Arges. - - Seb. mulf.2.t. I03.f. I.

Habitnt in Africa.

Occiput bilubo gibbum. Corpus ocelis transuer/is per annulus digeflis. Mibi non vifus, nec jeuta notata.

I I0. ANGVIS. Squamae abdominales et Siguanuce fubcaudales.

160. bipes. 100-60. Muf: Ad. Ir. I. p. 21. t. 28. f. 3.

Seb. minf. I. t. $53 . f .8$.

Habitat in Indiis.

t. $86 . f \cdot 3$.

Pedes 2 breuiffini, didactyli, ad anum.

Pallida puncto fuico in fingula Squama.

197. Melea. 165-32. gris.

Scb. muf. 2. t. 2I. f. 4 .

Habitat in Indiis.

Sinnilis $A$. bipedi, glauca punctis nigris multiplici ordine longitudinali.

P 4

198.

COLVBRI a me non vifi, a Cl. GRONOV10 in museo 2. deferipti.

165 abd. 141. caud. 24 Gron. 41. Scb. 2. t. 98. f. 1.

175 - 136 - 39. Gion. 38. ferrngineo cnernleo alboque variks.

177 - 135 - 42 Gron. 3y. allus maculis albis nigrisque.

201 - 159 - 42. Gron. 29. Sch. 1. t. 33. f. 6. albo-rufus.

202 - 142 - 60.Gion. 36. Seb. 2. t.35 f. 4. caerulejcens.

203 - 153 - 50. Girno 34 . albus lincis maculisque rigris.

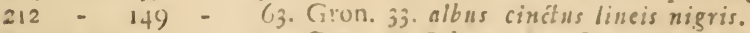

213 - 139 - 74. Gron. 37 Seb.2.t.20. f. 2 caerulens maculis nigris.

220 - 160 - 60.Gron. 28. cantitus Intis albis nigrisque.

$230-165$ - 74. Gron. 25. Scb. 2. t. 21 . . 3. albus fafciis ol. scuris.

234 - 174 - CJ. Gion. 22. Scb. 2. t. 33. f. 1. cizereo cuernleus.

$210-163-77$. (iron. 26. Seb. 2. t. 1. f. 9. et t. 9. f.2. vitta nigra.

260 - 180 - 80. Gron. 20. albo binnseoque zarius.

266 - 1y1 - 75. Citon. 15. brunneus pundis albis.

2,8 - 202 - 9f. Gion. 14. moculis lirunueis.

311 - 189 - 122. Gron. 17. purfurafcens macslis atris.

$314-172$ - 142. Ciron. 23. caevoleus linea laterali atra.

315 - 190 - 125. Ciron. 29. cinctus lineis albis nigrisque.

$j+2$ - 272 - 70.Gron.11. Seb. 2. t. 199. f. z. Mclulatxs. 
198. colubri- I80-I8. Haffelqu. iter. 320. 72.65. nus.

209. Taculus: 186-23. Hafelqu. iver. 319. n. 64.

Habitat in Aegypto.

Squanae audominis paulo latiores.

212. maculata, 200-I2. Mul: Ad. Fr. I. p. 2r. t. 2r. f. 3 .

Gron. mie. 2. p.53. n.5.

Seb. manj:2.t. I00. $f: 2$.

$$
\text { I. } t .53 \cdot f \cdot 7 \text {. }
$$

Habitat in America.

Supra flana, vitta dor-fali fafciisque linea. viuus fugcis:

214. reticulata. $177-37$. Gron. mus. 2. p. 54.n. 7 .

Scheuch. ficr.t. $747 \cdot f \cdot 4$.

Habitat in America. i

Color fquanarum fufcus difco alba.

215. Ceraftes. 200-15. Haffelqu. Act. Vpf. $1750, p \cdot 28$. it. 320. 11.66.

Habitat iza Aegypto. $\dot{\dagger}$

237. lumbrica-230-7. Gron. mul. 2.p. 52.n. 3 . lis:

Brovn. iam. 460 .t. $4 \frac{4}{4}$. . 1 .

Seb. mil. . p. 137.t. 86. $f, 2$.

Habitat in America. $\dagger$

Color ex albida flauffcens.

250: laticauda, 200-50. Mi:f. Ad. Ir. 2. p...

Habitat Surinami.

Couda conpreffa, acuta, pallida fafciis fujcis.

253. Scytale. 240-I3. Amocn. acad. r. p. 296. Mitf: Ad. Fr. I. p. 21. t. 6.f. 2.

Gron. me. 2. m. 4. Anguis 227-I4,

Seb. mmlt: 2. t. 2. f: $1,2,3,4$.

$$
\begin{aligned}
& \text { t. } 7 \cdot f \cdot 4 . \\
& \text { t. } 20 . f .3 .
\end{aligned}
$$

Habitat in Indiis.

Albida fquamarum marginibus pafjun ferługineis, fajciis fuscis. 


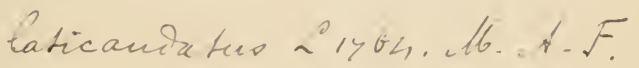




262. Eryx.
Gron. mu!f. 2.p. 35.n. 9. (126-136.)
Hubitat in America. (non vicii.)
Cincrea lineis tribus nigris; fubtus caeru- lejiens.

270. Fragilis. I35-135. Faun. face. 258 .

Aldr. Jerp. 245 . Caecilia vulgaris.

Habitat in Europa.

Huius maxima fragilitas lepide explicata a Lemery in dictiontario.

Ir. AMPHiSB AENA. Aimtli trunci caudaeque.

230. fuligino- 200-30. Anven. acad. I. p. 295.

f., Miv. Ad. Fr. I. p. 20.

Gron. mulj 2.p.r. Amphisbaena 209-25. Rai. quadr. 288 .

Seb. muj:2.t. I. f. 7 .

$$
\begin{aligned}
& \text { t. } 18 . f .20 \\
& \text { t. } 22 . f: 3 \\
& \text { t. } 73 . f: 4 \\
& \text { t. } 100 . f \cdot 3 \\
& \text { I. t. } 88 . f .3
\end{aligned}
$$

Haüitat in America.

illbo nigrogue ravia.

239. alba. 223-16. Muf. Ad. Fr. I. p. 26.t. 4.f. 2 ! Seb. mil: 2. t. 24. f. I.

Habitat in America.

$$
\text { t. } 6.1 .4
$$

Alba tota.

12 CAECILIA. Rugatrunci caudaeque. Lubium fiperius tentaculis 2.

135. tentacula- 135-0. Anocn. acad. I. p. 489. t. 17.f. 2. ta. Muf: Ad. Ir. 1. p. 19. t. 5.f. 2 . Grom. mul: 2. $\%$. 1. p. 52 . Habitat in America.

350. Glutinofa. 340-10. Mul: st. Ir. I. p. I9. t. 4.f. I, Habitat in Indiis.

lufia, linea latcrali albian.

$$
P_{j}
$$




\section{NANTES.}

Spivacula lateralia.

Pinzac natatoriace.

I13. PETROMYZON. Spiracula VII ad latera colli.

Fiffula in vertice.

Pinnae pectorales aut ventrales nuilae.

marinus. I. P. ore intus barbato, pinna dorfali pofteriore a cauda diftineta.

Art. gen. 64. Jyn. go. Petromyzon maculofus, ordinibus dentiun crrciter viginti.

Bell pilc. 76. Muftela î. Lampetra.

Gefit.pifc. 590. Multela f. Lampetra.

Rond. pifc. 398. Will. icht. 105. Rai. pifc.35. Lampetra.

Habitat in Marvi Europaeo.

fluuiati- 2. P. pinna dorfali pofteriori angulata. Fn. fuec. 273.

lis. Art. gen. 64. lyn. S9. Jec. 99. Petromyzon vnico ordine denticulorum minimorum in limbo oris praeter inferiores maiores.

Gefis. pifi. 597. Lampredae alterum genus.

Saí, pifi. 63. Pryck.

Ionft. pifc. t. $24, f .6$. Pricka.

Will. icht. 106. Rai.pifc. 35. Lampetrae medium genus. Fiditat in fumiis Europae.

sidhacet ove, nowibus tenebricofis cono cauo pifcatur.

branchi- 3. l'. pinna dorfali pofteriore lineari, labio oris fuperiore alis. Hatere lobato. Fin. fuec. 274 .

Rond. pifc. 202. Giefr.pifc. 598. Will. icbt. 104. Rai. pijc. 35. Lampetra parua et fluuiatilis.

Sal\% pifi. 63. Lamperra minor.

Hobit at in aquis dulcibus Europae, brancbiis pifcium inbacrens.

I 4 . RA-

Fetiognyzon goudet perfedzoribus pulmonibus, qaam reliqui ordinis, adeoque ferpentibus propior. 

Raia seabra A.1464.- ab. A. F.p. 52 


\section{Raja}

I 4. RAIA. Spiracula V fubtus ad collum.

Corpus depreffum.

Os fub capite.

* Dentibus acutis.

Torpedo. I. R. tota laeuis. Art. gen. 73. $\int y n$. 102. Antocn acad. I. p. 307. Muf: Ad. Tr. I. p. 52.

Bell. pifc. 89. 90. Rond. pifc. 358. Sult. pifc. 142. Gc/n. pific. 998. Rai. pifc. 28. Torpedo.

Kaemipf. exot. 509 . t. 5 10. Torpedo.

Habitat in Sinu Perfico, M. Mediterraneo.

Ine;s, onniun iniuriac obnoxius fe vindicat motu tremulo proprio. Act. baffin. 5. obf: 97. Rhed. exper. 53 . Kaempf. amoen. 5I4. Reanm. act. I714. Hall. boerb. 4. p.I3. retardato fFiritu praejeruatur.

Batis. 2. R. varia, dorfo medio glabro, cauda vnico aculeorum ordine. Art. gen. 73. Jìn. IO2.

Rond. pifc. 346. Gefin. pifc. 791. Rai. pifc.25. Raia vndulata f. cinerea.

Ionft. pifc. Raia vndulata.

Habitat in Oceann Europaeo.

Oxyzinchus. Corpus Jipra litwris nigris flexuofis.

Oxyrhyn-3. R. varia, dorfo medio tuberculis decem aculeatis. Art. chus. geil. 72. Jin. IOI.

Salu. pijc. 149. Laeui-Raia.

Gifin. pijc. 792. Will. iclst.77. Rai. pifc. 26. Raia oxyrliynchos maior.

Habitat in M. Mediterraneo et $O$. Europaeo. Aculcus ante fingulos oculos.

Mirale- 4. R. dorfo ventreque glabris, aculeis ad oculos, ternotus. que corum ordine in cauda. Art. gen. 72. Syn. IOI. Salı. pifc. 150. Raia ftellaris. Ionft. pifi....t. ro. f. 4. Raia oculata. Will. icht. 72. Rai. pifc. 27. Raia lacuis oculata, Habitat in mari Nediterraneo. Ocellus Jupra vtramque alam.

fulloni- 5. R. dorfo toto aculesto, aculeorum orline fimplici ad

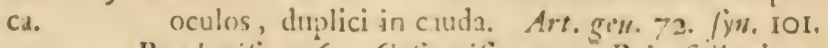
Rond. pifi. 35 6. Ciefir.pifc. 797. Rais fullonica. 
Will. icbt. 78. Raia fullonica; Raia aípera. Rai. pijc. 26 . Raia afpera. Habitat in M. Europaeo.

* * Dentibus obtufis.

Aquila. 6. R. corpore glabro, aculeo longo ferrato in cauda pinnata. Art gen. 72 . $\int y n$. 100 .

Salu. pifc. I46. Rond. pijc. 338. Bellon.pifc. 97. Gejin. pifc. 75 . Aquila marina.

Rai. pifc. 23. Aquila.

W'ill. iclst. 64. Aquila.

Column. ecplbr. I. p. 3. t. 2. Paftinaca marina laeuis altera oxypterydes.

Ilabitat in Mari Mediterraneo.

Altaukla. 7. R. corpore glabro, aculeis duobus poftice ferratis in cauda apterygia. Art. gen. 7 I. $\int j n$. IO9.

Column. cepbr. I. p. 4.t. 2. Will. icbt. 65. Rai.pifc. 2.. Paítinaca marina altera pteryplateia f. Altauela. Habitat in Mari Mediterraneo.

Cauda corpore brecuior eft.

Paftina- 8. R. corpore glabro, aculeo longo anterius ferrato in ca. cauda apierygia. Art. gen. 7I. Jyn. 100.

Bell. pifć. 94. Paftinaci marina laeuis.

Rond. pifi. 33I. Salu. pifc. I44. Paftinaca.

Gelin. pijc. 679. Paftinaca marina.

Will. icbt. 67 . Paltinaca marina $\mathrm{I}$.

Rai. pifi. 24 . Paftinaca marina laeuis.

Habitat in Europa.

Caudac aculeus venenatus veteribus et recentivribus. Fiato Vlyijis clarus.

clauata. 9. R. aculeata, dentibus tuberculofis, cartilagine transnerfa ventrali. Art. gen. 71. Jyn. 99. Jpec. I03. Fn. Jilec. 268 . It. W got. 175 .

Rond. pifc. 353. Gefin.pifi. 795. Raia clauata.

IVill. icbt. 74. Rai. pifc. 26. Raia clauata.

Habitat in Occano Europaeo.

Rhinob2. IO. R. oblonga, vnico aculcorum ordine in medio dorfo. tos. Art. gen. 70. Jyn. 99. 




\section{AMPHIBIA NANTES. Raia.}

Bell. pifc. 78. Gefn. pifc. 903. Squato-Raia f. Rhinobatos.

Salu. pifj.153. Will. icht.79. Rai.pifc.28. Rhinobatos f. Squatino - Raia.

Habitat Genure, Venetiis.

I j. SQVALVS. Spiracula V ad latera colli. Corpus oblongum teretiufculum. Os in anteriore capitis parte.

* Dorfo spinofo: pinna ani mulla.

Acan- I. S. pinna ani nulla, dorfo fpinofo, corpore teretiufculo. thias. Art. gen. 66. Jin. 94. Jjec. I02. Fn. Jikec. 269 . Mur. Ad. Fr. I. p. 53. It. WVgrt. 174 .

Rond. pifc. 373. Galeus acanthias.

Gefir. pifc. 60\%. Galeus acanthias.

Bellon. pifc. 69. Muftelus fpinax.

Salut. pifc. I35. $f$. I36. Muftelas fpinax.

Will. icht. 56. Galeus acanthias f. fpinax.

Rai. pifc. 2r. Gaieus acanthias f. 1pinax.

Habitat in Oceano Europaeo.

Centri- 2. S. pinna ani nulla, dorfo fpinofo, corpore fubtrianna. gulari. Avt. gan. 67. 11.5. Jjn. 95.

Rond. pifc. 384. Centrina.

Salu. pije. $156-158$. Centrina.

Ge/in. pifć. 609. Centrina.

Will. icbt. 58. Centrina.

Rusi. pific. 2I. Centrina.

Mabitat in Mari Mediterraneo.

Pinna dorfi anterior jpina antrorfam radios deculfante, poflwior retionsunth.

Spinax. 3. S. pinna ani nulla, dorfo fpinofo, naribus terminalibus. Art. gen. 67. Jin. 95 .

Will. iclst. 57. Faleus acanthias $f$. fpinax fufcus. Rai. pific. $2 \pi$. Galcus acanthias $f \cdot f_{p}$ inax fufcus. Habitut in Europa.

Squati- 4. S. pinna ani nulla, ore terminali. Art. gen. $67 . n .6$. na. (in. 95. licillon. pifi. 78. Siquatina. Rundel. pifc. 367. Squatina. 
Gefin. pifc. 899. Squatina.

Will. iclst. 79. Squatina.

Rai. pifc. 26. Squatina.

Habitat in Oceano Europaeo.

Media inter Squalos et Raias, ore in apice capitis, nec jubtus.

* * Dorfo matico, dentibus acutis; cum pinna ani.

Zygaena. 5. S. capite latifimo transuerfo malleiformi. Art. gen. 67 . jyn. 96. Muf. Ad: Fv: I. p. 52. *

Bell. pifc. 6o. Zygaena f. Libella.

Rondel.pifc. 389. Zygaena.

Gejn.pifc. 1050. Zygaena.

Salu. pifc. I28. Libella.

Will. icht. 55. Zygaena.

Rai. pijc. 20. Zygaena.

Habitat in Europa, America.

Tiburo. 6. S. capite latiffimo cordato.

Will. icbt. 55. Zygaenae aftinis, capite triangulo.

Marcgr. braf. I8I. Tiburonis fpecies minor.

IIabitat in America, praecedenti affinis.

Galeus. 7. S. naribus ori vicinis, foraminibus ad oculos. Art. gen. 68. $11.9 \cdot$. Jyz. 97.

Rond. pifc. 377. Galeus canis.

Gefin. pific. 167. Galeus canis.

Salu. pifc. I30. Canis galeus.

Will. icht. $5 \mathrm{I}^{\cdot}$ Canis galeus.

Rai. pifc. 20. Canis galeus.

Habitat in Oceano Europae.

Canicu- 8. S. rufo-varius, pinna ani medio inter anum caudamla. que pinnatam. Art. gen. 68. (ju $u .97$.

Osbeck. iter 70. Squalus conductus.

Rond.pifc. 380. Canicula ariftotelis.

Gefir. pific. I68. Canicula ariftotelis.

Salu. pilc. . . Catulus maior.

Will. iclst. 62. Rai. pifc. 22. Catulus maior vulgaris.

Habitat in Oceano Europae.

9. $S$.

Squali vinipari funt, foramine in ore aperto, pone vtrumque oculum, noctu Splendent, refupinati vorant, naues Jequuntкr in Indias vt cadaueribus jaticntur. Dentes Glofopetrae. 


Catulus. 9. S. dorfo variegato mutico, pinnis ventralibus concretis, dorlalibus caudae approximatis. Art. gen. 69. Jjn. 97.

Gron. mul. 2. n. 199. Sqqualus idem.

Salu. pifc. 137. Cutulus minor.

Uill. icht. 34. Carulus minor.

Rai. pijc. 22. Catulus minor vulgaris.

Habitat in Oceano Europae.

ftellaris. I0. S. varius inermis, pinnis ventralibus difuretis, dorfalibus caudae approximatis. Ait. gin. 69. Jyn. 97 .

Gron. mus 2. 11. 200.

Will. icht. 63. Carulus maximus.

Rai. pijc. 22. Catulus maximus.

Habitat in Mari Europaeo.

glaucus. II. S. foffula triangulari in extremo dorfo, foraminibus nullis ad oculos. Art.gen. 69. n. I3. $\int_{3} n .98$.

Rond. pifc. 378. Gicfin.pifs. 609. Will. icbt. 49. Rai. pific. 20: Galeus gliucus.

Habitat in Oceano Europaeo.

Carcha- I2. S. dorfo plano, dentibus ferratis. Art. gen. 70. Jyn. rias. $\quad 98$.

Rond.pifc. 490. Lamia.

Gicin. pijc. I73. Will. icbt. 47. Rai.pifc. Is. Canis Carcharias f. Lamia.

Habitat in Europa.

Ionam Proplotan, vt veteres Herculen trinosem, in buius ventriculo tridui fpatio, baciije verofimile eft.

*** Dintibus granulatis.

Mufte- I3. S. dentibus obtufis. Art. gen. 66. Jyn. 93 .

lus. Gefin.pilc. 608. Rond. pifc. 375. Galaeus lacuis. Saltup pilc. 135, 137. Muftelus laeuis.

Will icht. 60. Rai. pijc. 22. Mufteius laeuis primtss. Hiabitat in Europa.

Prifis. I4. S. rnflo cenfiformi offeo plano vtrinque dentato. Art. gen. 66. Jinn. 93. Jiunn. Jikec. 270. Mul: Ad. Fr.

I. $p .52$. Siqualus roilro longo cupidato ofleo plano verinutue dentato.

Biellow. pilc. 66. Serra marina.

Rond. pifc. 487. Prillis. 
Clat. exot. I36. Priftis R. Serra pifcis. Will. icht. 6I. Rai. piff. 24. Priftis r. Serra pifcis. Habitat in Europa.

116. CHIMAERA. Spivacula folitaria fub collo. Oris labium fuperius bipartitum. Dintes primores incifores, bini fupra infraque.

monftro- r. C. roftro fubtus plicis pertufis.

f.. Muf: Ad. Fr. i. p. 53.t.25. Chimaera. Clail: exot. 136.t.137. Genus Galei. Will. icht. 57. Galeus acanchias clufii. Rai. pifc. 23. Galeus acanthias. Habitat in mari Atlantico.

Callo- 2. C. roftro fubtus labro inflexo laeui. rynchus. Gron. mul: 59.n. ro. t. 4. Callorynchus. Frefier. itin. I. p. 2 m. t. I7.f.4. Peiegallo. Habitat in mari Aethiopicu.

117. LOPHIVS. Spiraculd folitaria, pone brachia. Pinnace pectorales brachilis infidentes.

pifcato- I. L. depreffus, capite rotundato.

rius. Art. gen. 63. Jyn. 87. Lophius ore cirrofo. It. jécan. 327. Lophius ore cirrofo. Muf: Ad. Fr. 55. Lophius ore cirrofo. Gron. mutf. I. P. 57 . Lophius ore cirrofo. Cbarl. onvonn. I99. t. 20I. Rana pifcatrix. Olear. mul $37 . t .23 . f .4$. Rana pifcarrix. Habitat in Ocemo Europaeo, fit lapidun fornicibus. Pinna D. Io. P. 24. V. 5. A. 9. C. 8 . Caput corpore maturs. Pifcatur tintaculis binits capitis tainquanin lizes.

Vefperti- 2. L. depreffur, capite roftrato.

lio. Art. Jim. 88 . Lophius fronte vnicorni. Muf. Ad. Fi. I. $p .55$. Lophius fronte vnicorni. 




\section{AMPHIBIA NANTES. Lophius.}

Gron. mul. x. "1. 129. Lophius fronte vnicorni.

Seb. muf. I. p. IIs. t. $74 . f .2$. Rana pifcatrix ameri. cana.

Marcgr. braf. I43. Rai.pifc.30. Guacu-cuia,

Habitat in Ocenno Americano.

Pianae D. 5. P.jo. V. 6. A. 0. C. 15.

Confer. Brown. ianm. $157 \cdot$ t. $48 \cdot f \cdot 3$.

hiftro. 3. L. compreffus.

Cbin. Lagerftr. 21. Lophius pinnis dorfalibus tribus. Minf: Ad. Fr. I. p.56. Balifes f. Guaperua chinendis. It. Wgotb. I37. t. $3 \cdot f \cdot 5 \cdot$ Baliftes $\mathrm{f}$. Guaperua. Marcgr. braj: 150. Guaperua. Will. iclbt. 50. t. E. 2. f. 2. Guaperua. Osb. iter. 305. Lophius tumidus. Ilabitat in Pelago inter Fucum natanten. Pinna: D. I, I, 12. P. 10. V. 5. A. 7. C. I0.

II S. ACIPENSER. Spiracula lateralia folitaria, linearia.

Os fub capite, retractile, edentulum. Cirvi fub roltro, ante os.

Sturio. I. A. citris 4 , fquamis dorfalibus Ir. Muj: Ad. Fr. I. p. 54. t. 18.f. 2 .

It. Scan. 187. Acipenfer.

Art.gen. 65. Jya. 91. Acipenfer corpore tuberculis fpinotis afpero.

Fn. fuec. 27. Acipenfer idem.

Gron. muf: 2. p. 42. Acipenfer idem.

Habitat in muri Luropaco.

Plunae D. 31. P. 30. V. I9. A.24. C.24.

Seurri imp. acun a coronotis miniftris praceunte tibicine ad epulas adjportabatur.

ruthenus. 2. A. cirris 4 , fiuumis dotfalibus 15. Muf. Ad. Fr. I. p. 54.t.27.f: 2. et t. 2S. f: . .

Fin. fisc. 272. Acipenter ordinibus 15 fyuamarum offewrum: intermedio ofinculis 15 . 
238 AMPHIBIA NANTES. Acipenfer.

Habitat in Rutheno; introductus a Rege Friderico I in Maelerum Sateciae.

Oua buius et Hufonis pro Garu Cavear dicto.

Hufo. 3. A. cirris 4 ? corpore nudo.

Art. gen. 65 Jyn 92. Acipenfer tuberculis carens. Habitat in Danubio, fluniisque Imperii Ruthenici. Alii cirros 4, alii 8 tradunt in nobis non vifo. Corium pro lineis currum praeftantiffrmun; ab co Icbthyocolla praeparatur et ex ouis Garum conficitur.

Plecolto- 4. A. cirris duobus. Mul. Ad. Fr. I. p. 55. t. 28. mus. f. 4 . Gion. mur. I. n. 67. t. 3. f. I, 2. Plecoftomus dorfo dipterygio, cirris duobus, cauda bifurca. Habitat Surinami.

Pinnae D. 8, I. P. I. V.6. A. 5. C. 12.

Acipenferes ouipari funt, wti Petromyzon, 

Pisces 2. 1735.- 


\section{CLASSIS IV. \\ P I S $, \mathrm{C}, \mathrm{E} S$.}

A QVEI Elementi muti furdique Volucres compendicfifimi refpirant Brancbiis liberis, natant Pinnis radiatis, veftiuntur Squamis cartilagineis.

INSTRVVNTUR Capite, Thorace, Abdomine, Cauda, Pinnis; Cerebro Medullaque fpinali, Corde vniloculari vniaurito, Branchiis, Diaphrigmate, Hepate Veficulaque fellea, Liene, Renibus Veficaque vrinaria, Veficulis feminalibus Ouariisue, Inteftinis caecoque, Membrana niEtitante.

DESTITVVNTVR Palpebris, Auribus Auriculisque, Collo, artuum Brachiis Tibiisque, Pene Vuluaue.

DIVIDITVR horum Corpus externum in Caput, Truncum, Pinmas.

CAPVT compreffum (Cathetoplateum) depreffum (Plagioplateum ).

Os Labiis mobilibus, aut fixis claufile.

Dentes frepe in Maxillis, Lingua, Fauce.

Oculi 2. nudi, membrana nicfitante; Pupilla globofs. Nares pertufae, fuepe geminatae minus prominentes.

Cirri filiformes, molliufculi, labiis interủum inferti.

Apertarrae Branchiarum per branchias in os hiantes.

Opercula branchias tegentia, aperturas claudentia.

Brancbiae extus fpirantes comprimendie, faeplus quatuor, radio arcuato ofleo adnatac, radis liberis peetunatae.

TRVNCVS fquamofus, mucofus, oblongus, poftice anguftarus in Caudam: Squamae pierisque cartilagineae, rarius ciliatae, nonnullis offeae vt pifcis loricatus euadat. 
Linea lateralis vtrinque diftineta a linea interftitiali, fae. pius ex glindulis punciata, rarius loricata, vtraque reita aut curuata.

Thos ax refertus Corde intra Pericardium fuum.

Abdonen coftatum Vifceribus, Inteftinis, Veficula ä̈rea. Anus abdomen terminans, communis Inteftinis, Veficae et Genitalibus.

Cauda poftica Trunci, folida, mufculofa.

PINNAE membranaceae expandendae Radiis cartilagineis, qui Spinofi duriores, offei, fimplices, pungentes. $\left({ }^{*}\right)$ Mulles flexiles, tendinei, dichoromi, mutici.

Branchiof?egae fub operculis branchiarum plicatiles radiata membrana.

Dorfalis dorto longitudinalis impofta, fuepe I, rarius 2 , paucifimis 3 .

Adipoja, dorfalis puria, radiis deftituta.

Pectorales ad lacera thoracis politae.

Ventrales abdomini fubous annexae.

Analis pone anum, fub cauda, loncitudinalis.

Caudalis rerminalis, perpendicularis: rotunda, integira, 1. bifurca.

PISCES hi muti furdique aetheris fonum non audiant, aëris licet tremorem fentiant; Rejpirant latere extrorfum aquam; Monent fe pinnis, dum Caudali propelluntur, Dorgli Analique tamquam Gubernaculo reguntur, Pectoralibus tamquam Alis attolluntur, Ventrolibus tamquam Pedibus inlifunt; Fugiunt natatu diuerfimo. do; Defenderlutur. Spinis tamquam murice; Victitant Muco, Infectis, Vermibus, Cadaueribus, Pifciculis, Plantis.

Hifloria horum ex Strugura, Alimento, Solo, Moribus, Migrationibus et Vfu in Pifcatura, Culina, Oeconomia, Mechanica, Medicina.

LOCVS omnium aquaticus eft; forte etiam omnes marini; fed alii imprimis lacufres aquas dulces inhabirantes; varii etiam fluiatiles, iaepe e mari adicendentes; plurimi mere

(") Recumbens Jpina nonrullis antrorfum decnmbit ante pinnam dorfin ant ani. 


marini, qui aquas dulces non ferunt, horum plerique iita tora adcunt pro generandis $\left(^{*}\right)$ et pariendis nuis, calidiore aqua exciudendss, nili mere peíngici natantibus ouis, a littoribu; fenper alieni, led omnium alii diumi, alii noctumi illi plerique natant in aquis, hi vero faepius in fundo pafcuntur. Alii demum fubfolitarii vagantur, alii gregatim dezunt.

YICTVS tenuis e pifcibus nuper enętis, tinoracicis, exercitatis; imprimis afliti, veteribus in pretio, hodiernis in deliciis.

ICHTHYOLOGT Prakici Bellonius, Pondeletius, Saluianus, Gefuerus, Aldrouandus, Willugbuaeus, Raius.

Theoretici Artedi! Nos, Gronouii, Haffelquift, radios pinnarum obferuarunt; At Valentinus defectum artis expofuit; Catesbaeus pulchre quosdam depinxit americanos.

Synonyma ab Artedio petenda, eร̧o itaque ea fepofui.

DIVISIONEM Pifcium, artis normam, a variis partibus tentarunt varii. Recentiorum immortalis Artedi Spinofos Acanisopterygios er Inermes Malacopterygios fecundum Membranam Brancbioftegam fecutus, fed deferuit non femel in biuio conflitutum; nouam itaque tentabo viam a PEDIBVS ante Alas, jub Alis, pone Alas titis.

Apertura infructa operculis pinnaque branchiali : dum

Pinnae ientrales: vel mullae omnino A PODES. I.

- - ante pinnas Peftorales IVG VLARES. 2.

- - jib pinnis Petoralibus THORA CICI. 3 .

- - pone pinnas Peitornes ABDOMINAL.E. 4 .

- Deftituta operculis pinnisue branchialibus BR $1 \mathrm{~N}$ CHIOSTEGI. 5 .

\section{Q 3

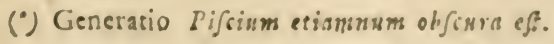




\section{CHARACTERES PISCIVM.}

\section{A PO DES.}

II9. MVRAENA Aperturae brancbiarum ad latera thoracis.

I2O. GYMNOTVS Dorfum atterygium.

I2I. TRICHIVRVS Cauda apiera.

122. ANARHICHAS Dentes rotundati.

123. AMMODYTES Caput corpore anguftius.

124. STROMATEVS Corpus ouatum.

125. XIPHIAS Roftrum enfiferum.

\section{I V G V L A R E S.}

126. CALLI ONYMvS Aperturae branch. ad micham. 127. VRANOSCOPVS OS fimlinl.

129. TRACHINvS Anus prope pecius.

129. GADVS Pinnae Pectorales elongatae in acumen.

130. BLENN I vS Pinnae Ventrales didactylae muticae.

I3I. OPH IDION Pinnae Ventrales é fpina et cirrbo.

\section{THO R A C I C I.}

132. CYCLOPTERVS Pinnae Ventrales connatae in orbiculum. 133. ECHEN E 15 Capitis dorfum planum transuerfe fulcatum. 134. CORYPHAENA Caput antice trincato-obtrafum.

135. G О В I VS Pinae Ventrales coadunatae in pinnam ouatan. 336. Cотtvs Caput corpore latizs.

137. SCORPAENA Caput cirris adfperfum. 138. ZEVS Labium fuperius membrana transuerfa fornicatum. 139. PLEVRONECTES Oculi ambo in latere altero capitis. i40. CHA ETODON Dentes fetacei, confertiffnit, flexiles. 14I. SPARvS Dentes validi, incifores f. molares.

142. LABR V S Pima Dorfalis rameato poft fpinas notata. 143. SCIAENA Pima Dorfalis in fofula recondenda. 144. PERCA Opercula brancbiarum ferrata.

145. GASTEROSTEVS Cauda lateribus carinata; Spinae dorfales diftinctae. 

162. Cormurus L. pornit inter, "Abdominales" 1764 tono. Ad. Frid.

163 Salistes 165 tetrodon Sinnaenopont inter" Auphibia Nantia 16 gegasus illes. At. Frid. $1964 .-$ 
I46. Sc о 147. MVLLVs Squanae, ctiam capitis, laxae.

I48. TRIGLA Digiti diftincti iuxta pinnas pectorales.

\section{A B DO MIN AL ES.}

149. COBIT Is Corpus vix ad Caudam anguftatum.

jjo. Silvrvs Radius I pinnarum Durfalium L'ectoraliumque dentatus.

15I. LORICARIA Corpus cataphractum.

152. Sa L mo Pinna Dorfalis poftica adipofa.

153. FISTVLARIA Roftrum cylindricum operculo clanfile.

154. ES OX Mandibula inferior longior punctata.

155. ARGENTINA Anus caudae vicints.

156. ATHERINA Fafcia lateralis longitudinalis argenten.

I57. MVGIL Mandibula inferior intus carinata.

158. EXOCOET V Pinnae Pe Ctorales longitudine corporis.

I59. POLYNEMVS Digiti diftincti iuxta pinnas pectorales. 160. CLVPEA Abdomen carinatum ferratum.

161. CYPRINVS Membrana Branchioftega triradiata.

\section{BR A N C H I O S T E G I.}

162. MOR M Y R V S Dentes emarginati. Squamae imbricatae. 163. BALIStes Dentes vtrinque vĩo. Pinna Ventralis jolitaria. 164. OSTRACION Dentes vtrinque plures. Corpus tefta offer. 165. TETRODON Dentes vtrinique dun. Abdomen muricatum. 166. DIODON Dentes strinque folitarii. Corpus echinatum. 167. CENTRISCvS Pinna Ventralis Jolitaria. Corpus loricatume Jpina.

168. Syncinativs Pinnae Ventrales mullae, Corpus articulatum.

169. PEGASVS Pinnac Ventrales binac. Corpus articulatum. 


\section{APODES.}

I19. MVIAENA. Caput laeue. Nares tubulofae. Membrana branchioflega radiis $\mathrm{X}$, eiusque Apertura ad pinnas pectorales.

Corpus teretiufculum, lubricum. Pinna caudae coadunata dorfali anique. .

Helena. I. M. pinnis pectoralibus nullis. Art. aen. 25. (jnn. 4r. Gron. mul. I. n.15́. Muf: Ad. Fr.

- . p. 319. Muraena pirnis pectoralibus carens.

Seb. mulf.2.t. 69. f. 4, 5 .

Catesb.car.2.t. 20, 2I. Muraena maculata nıgra, et viridis.

Habitat in Europae, Americae Oceano.

Caenumun Helena, Romae in pifcinis: natat ad magiJtrun delicata Muraena, Martial; Ved.Pollio dannata mancipia inmergebat vinariis Muraenavun, vt in vifceribus, fuis aliquid de Seruorum fitorum corpore guffaret. Plin. IK: 23. Morju infefta.

Ophis. 2. M. cauda aptera cufpidata, corpore tereti.

Art. gen. 24. Jyn. 4I. Muraena teres gracilis maculofa, cauda tereti cufpidata apterygia.

Habitat in Oceano Europaeo.

Serpens. 3. M. cauda aptera acuta, corpore rereti.

Art. gen.24. Jyn. 4r. Murae-

na exacte reres, cauda acu-

ta apterygia.

D..-P.16.V..-A..- C.-Habitat in Oceano Europae auftralis. 


Anguil- 4. M. maxilla inferiore lon-

la. giore, corpore vniculore. D. 1000.P.I9. V. O.A. 100.C.Art. Jpec. 66.gen.24. lin. 39. Fn. Jiec. $290 . \mathrm{Mu}-$ raena vnicolor, maxilla inferiore longiore.

Habitat in Europa; maxima in lacu Cornacbio Ferrda ricnfi; non fert Damubium.

Noturara; latet in coeno duplici foramine; coërcetur truncu albo Betulac; cutis tenacifina; parit viuipara, fub canicula. Act. Holm. 1750.p. 194.

Myrus. 5. M. pinna ambiente alba margine nigro.

Art. gen. 24. Jyn. 40. Muraena roltro acuto lituris albis vario, margine pinnae dorfalis nigro.

Ilabitat in Mari Mediterraneo:

Conger. 6. M. roftro tentaculis duobus, linea laterali expunctis albida.

Art.gen. 24. Jyn. 40. Muraẹna fupremo margine pinnae dorfalis nigro.

Habitat in Oceanso Europaco.

eseca. 7. M. apterygia, roftro acutiufculo.

Habitat in Mari Mediterraneo. E. Brander.

Corpus Anguillae absque ninni pinna. Aperturae brans cbiarum fub collo. Maxillue acuminatac dentibus acerofis. Nares tubulofae fiub roflio. Oculos nullos video; ad occiput punia 7 perforata ; itidem 7 Jupr caput in medio, et anterius iterun quater duo. Anus propior capiti quan cauda. 
I20. GYMNOTVS. Caput operculis lateralibus. Tentacula duo ad labium fuperius.

Membr. branch. radiis $\mathrm{V}$. Corpus compreffum, fubtus pinna carinatum.

Carapo. I. G. nudus, dorfo apterygio. Art gen.25. (jn. 43. Amoen. acad. I. p. 318.t. $14 \cdot j \cdot 6$. Mul: Ad. Fr. I. $p \cdot 76$. Gymnotus.
B.5.D. 0.P.13.V.0.A.200. C.0.

Habitat in America: Abdomen carnojo-carinatum; Pinna ani in caudac apicen excurrente.

afiaticus. 2. G. fquamofus, dorfo pin-
nato.
D. 46. P.14.V. O.A. 28. C. 16.

Habitat in Afia.

Pifcis plus quam fpitbameus, craffusculus, tectus fquamis lacuibus, rotundatis, diftinitis, etiam fupra caput, cui pun ta 5 excauata, vti etiam aliquot in Maxillis inferioribus. Anus in medio corporis. Einea lateralis eleuata, recta, jed dejeendens fupra anum. Color objourus fafciis fufcis. Puncta alba in pinna dorjali et poftica parte corporis. Ob opercula lateraliter anspla aperienda et ob fquainas nequit effe Muraena; pinna dorfalis incipit pone caput et extenditur fere ad caudam. Caput deprefJum, laeuiusfulum. Tentacula 2 truncata, ante nares pofita. Membr. branch. 5 . Corpus fubcylindricunn, poftice compreffum. Pinna dorfi mox poft nucban incipiens.

12I. TRICHIVR VS. Caput porrectum, operculis lateraibus. Dentes fubulati: anteriores conferti. Membr. branch. radio I. Corpus comprefio-enfiforme. Cauda fubuiata, aptera.

Lepturus. I. TRICHIVRVS. 


Art. Spec. III. Lepturus. B. 7. D.I00.P.II.V.0. A.105.C.0. Mul: Ad. Fr. I. p. 76.t.26.
f. 2. Lepturus.
B. 7.D. I24.P.I2. V.o. A. o. C.o.

Gron. mul:I. n. 47. Gymno-

gafter.

Brovon.iam.444.t.45.f.4.

B.7.D. I38.P.II.V.O.A. O.C.O.

Gymnogafter argenteus

compreffus, cauda atte-

nuata impinni.

Habitat in America, China.

Totus argenteus, exflilens ex aquis faepe in Cymban.

122. ANARHICHAS. Caput obtufufculum. Dentes confertiflimi, rotundati: antici VI, conici.

Membr. branch. rad. VI.

Corpus teretiufculum. Pinna caudae diftineta.

Lupus. I. ANArhichas. Art. gen. 23. Iyn. 38 .

Gron. muf. I. n. 44. Anarhichas.

Habitat in Oceano feptentriomali Angliae, etc.

Lapis Bufonites ex buius picis dente.

123. AMMODYTES. Caput compreffum, corpore anguftus. Labium fupe. rius duplicatum. Mandibula inferior angufta acuminata. Dentes acerofi. Memb. brancl. rad. VII. Corpus terectufculum fquamis vix confpicuis. Cont$d a$ diftincta.

Tobia- I. A MAnDYTES. Art. gen. I6.

nus. $\quad j$ j1. 29. Jjec. 55. B.7.D.54.P. 12. V.O. A.28.C.15. 


\section{PISCES APODES. Ammodytes}

Fn. furc. 303. Ammody-

tes. B.7.D.54.P.I3.V.0.A.28. C.I5. It. $\int c a i$. I4I. Ammody-

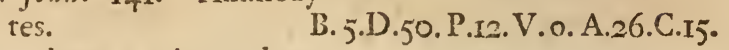

It. oel. 87. Ammodytes.

Muf. Ad. Fr. 1. p.75. Am-

$$
\text { B.7.D.60.P.15.V.0.A.32.C.14. }
$$
morlyies.

B.7.D.60.P.14. V.o.A.30.C.16. Habitat in Europae Oceano arenofo, in gyrun contractus, e centro apice capitis arenam penetrat.

124. STROMATEVS. Caput compreffum. Dentes in maxillis, palato. Membr. brancb. radiis. . . Corpus ouatum, lubricum; Cauda bifida.

Fiatola. I. S. fubfafciatus. Art. gen. rg. Syn. 33. Stromateus.

D.46.P.25.V.o.A. 34.C. - . Habitat in Mari Mediterraneo, of Mari Rubro. Pijcis pulcbre variegutus, ventriculi duo.

Paru. 2. S. vnicolor.

Sloan. iam.2. p.28I. t. 250. f. 4. Paru brafilienfi congener. Rai. pifc. 5 I.

Habitat in America.

25. XIPHIAS. Caput maxilla fuperiore terminatum roftro enfiformi; Os edentulum. Membr. branch. radiis VIII. Corpus teretiufculum.

Gladius. I. Xiphias. Art. gen. 30 .
Jin. 47.
Iingitat in Oceñno Eurnpae.
D. 4 I.P.17.V.0.A.15.C. $\therefore$
Scbellaneri Anatome Xipbii piscis. 




\section{I V G V L A RES.}

126. CALLIONYMVS. Caput labio fuperiore duplicaro; Oculi approximati.

Membr. branch. radiis V.

(Gron.) Aperturae laterales (faepius claufae, nucha foraminibus re(pirante).

Corpus nudum. Pinnae ventrales remotiffimas.

Lyra, $\quad$ r. C. dorfalis prioris radiis Inngitudine corporis. Muf:Ad.Fr.I. p.7I. Vrano copus.

Fn. fisec: 283 . Trachinus maxilla fuperiore longiore, pinna dorfi pricre altiffims.

Gron. muj: I. n. 64. Vranofcropus officulo primo pinnae dor falis longitudine entproris. D.4,I0.P.Ig.V.5.A.10.C. 10.

Gron. AA7 VPf: 174, O.p. I2I. t.S. Cottus officulis pinnae dorfalis longitudine corporis.

Act. and 293.p.1749. Gur-

D.4, ro.P. I8. V.6.A.10.C.I0. nardus lurens.

Habitat in Mavi Atlantico.

Dracun, 2. C. dorfalis prioris radiis culus. corpore trenioribus.

Gron. muf. 1. 11.63. Vranof:crive officulo primo pirnae dorfalis primae inciali. 
Art.gen. 49. $\int y n .77 \cdot$ Cottus pinna fecunda dorfi alba. D. 4, I0.P.12. V.6.A. 9. C.12.

Habitat Genuae, Romae.

indicus. 3. C. capite laeui longitudinaliter rugofo, operculis latere aperiendis. B.7.D. $\frac{\pi}{7}, I_{3} \cdot P \cdot 20 . V \cdot \frac{1}{6} \cdot A \cdot I 3 \cdot C . I r$. Habitat in Afia. Muf. Ac. Holmen]:

Capuc depreffun, fupra longitudinuliter rugofim. Maxilla inferior paulo longior. Os fcabrum. Lingua obtuja, emarginata. Aperturae brancbiarum magnae, laterales. Opercula anteriora jpina gemina; poftica fpina folitaria. Corpus valde depref]um, liuidum. Anus in medio. Radius primus P. dortalis prioris breuiffimus, remotus. P. ventrales valde diftantes. Pifcis medius quafi inter Callionymum, Vranofcopun et Tracbinum.

127. V RANOSCOPVS. Caput depreffum, fcabrum, mains. Os fimum: maxilla fuperiore breuiore. Membr. brancb. rad.V. papillofo-dentata. Opercula membranaceo-ciliata. Corpus: anus in medio.

fcaber. I. VRANOSCOPVS. Art. gen. 42. Jyn. 71. Trachinus cirris multis in maxilla inferiore. D.3,I4. P.16.V.5. A.13.C. -: Habitat in Mari Mediterraneo.

Caput fabrum, depreffum. l'inna dorfi prior nigra.

128. TR A CHINVS. Caput minus lacue, compreffum. Membr. branch. rad. VI; operculorum limina inferiore ferrata.

Corpus : anus prope pedtus.

Draco. I. TRACHINVS. It. $\int$ can. 325. 


Art.gent. 42. Jyn. 70. Fn. jiec. 282. Trachinus maxilla inferiore longiore, cirris deftstuta. D.5,3r.P.I6.V.6.A.32.C.I2.

Gron. mul: I. 12. 97. Idem nomen. D.6,30.P.15.V.6.A.34.C. Gron. ACt. VPS.1742.p.95. Idem nomen. $\quad$ D.6, 30.P I6. V. 6. A.30.C.I2. Habitat in Oceano Septentrionali.

Spinae P. durfalis prioris pungunt venenatiffmae. Barth. act. $3.0 b j: 84$.

329. GADVS. Caput laeue.

Memlir. branch. rad. VII, teretibus.

Corpus oblongum fquamis deciduis. Pinzale omnes cute communi vefitae: dorfales anique plures: radis miticis. Pectorales in acumen attenuatae.

* Pinnis dorfalibus tribus, ore cirrofo.

Aeglefi- I. G. tripterygius cirratus alnus. bicans, cauda biluba, maxilla fuperiore longiore. Fin. filec. 297.

Ait. gen. 20. Jyn. 36. Jpec. 64. Gadus dorfo tripterygio, ore cirrato, core pore albicante, maxilla fuperiore longiore, cauda parum bifurca.

Gron. muf: I. n. 59. Idem nomen.

It. Jcan. 325. Gadus Kolja.

It. Wgoth. 178. Gadus dor-

D. 15,20, 19. P. 18.V.6. A. 24, 21. C. . fo tripterygin, ore papillofo.

D. $16,19.20$. P. 17. V. 6. A. 22,21. C.23. IIabitat in Oceanu Europaeo. 
Callarias. 2. G. tripterygius cirratus varius, cauda integra, maxilia fuperiore longiore. Fn. fuec. 293. D. I5, I9,19.P.20.V.6.A.I8,I8.C.23. Avt. gen. 16. Jyn. 35, Jpec. 63. Gadus dorfo tripterygio, ore cirrato, colore vario, maxilla fuperiore longiore, cauda aequali. D.15, 19, I8.P.20.V.6.A.10,17.C... Gron. muf. I. n. 58. idem.

1t. oel.87. Gadus balthicus

$$
\text { D. I5, I9, I8.P. I0. V. 6. A.I6, I6. C. - }
$$
Torfk.

It. $\int$ can. 220. Gadus Cal-

larias balthicus. D. I5, I9, 17. P. 18. V. 6. A. I7,I6. C.24. Habitat in Oceano Europaeo et Balthico.

Morhua. 3. G. tripterygius cirratus, cauda fubaequali, radio primo anali fpinofo. Fn. filec. 295 .

Art. Syn. 35. Gadus dorfo tripterygio, ore cirrato, cauu่a aequali fere cum radio primo fpinofo.

1t. Wgotb. 176. Gadus Kab. beli:i.

D. I5.20.16.P.20. V.6. A. 2I, I6. C. =Habitat in Oceanu Europaeo.

Oua quotannis 9344000 ponit. Leeunenh.

lufcus. 4. G. tripterygius cirratus, radio ventralium primo fetaceo.

Art.gen. 21. fyn. 35. Ga-

D. I3,23.18.P.II. V. G.A.3I, I8.C.I7? dus dorfo tripterygio, ore cirrato, officulo pinnarum ventralium primo in fetam longam produeto.

Habitat in Oceano Europaco.

barbatus. 5. G. tripterygius cirratus, maxilla inferiore puntis vtrinque feptem. 


Art. gen. 2r. $\int y n .37 \cdot \int p e c$.

65. Gadus dorto tripte-

rygio, ore cirrato, lon-

gitudine ad latitudinem

tripla, pinna ani prima

ofliculorum 30 . D. I3,24,2I. P. I9.V.6.A. 30,21. C... Art. Jyn. III. Phycis (fecundum D. Kaeblerum).

It.Wgot.177. GadusTorfk.

D. I2,I8,I4.P.I4.V.5.A. $25,16 . C .30$.

It. Wgot. I78. Gadus li-

D. 12,20,20.P.IS.V. 6.A.I9.19.C.40. nea excauata pone caput.

D. $13,17,19$. P.18. V. 6.A.18, 17. C...

Habitat in Oceano Europaeo.

Fico Romanis bodie, cirro in mento, puncta maxillate inferioris vtrinque 7 ; macula fufca ad pin. pertorales. D. Kacbler.

minutus, 6. G. tripterygius cirratus, ano in medio corporis:

Art. gen. 21. Syn. 36. Gadus dorfo tripterygio, ore cirrato, corpore fefcunciali, ano in medin corporis.

D. $12,19,17 . P .13$. V. 6. A. $27,17 . C .-$ Habitat in M. Mediterraneo.

* * Irinis dorfalibus tribus, ore imberbi.

virens. 7. G. tripterygius imberbis, dorfo vireicente. Fin. Jitec. $296 . \quad$ D. $13,20,19 . P .17 \cdot V \cdot 6 \cdot A \cdot 24,20 . C .-$. Gron. AEZ.VPS.1742.p.90. Idem. ID.13,20,I9. P. 17.V.6.A.24,20.C. 40. Habitat in Oceano Europaer.

Merlan- 8. G. tripterygius imberbis a!gus. bus, maxilla luperiore longiore. Fin. Jitec. 294. Art. gen. 19. Jyn. 3t. liec.

62. Giadus dorfo tripterygio, ore imiserbi, corpore alto, maxilla lupe-

riore longiore. D. I4,21,20.P.21.V.6.A.33,23.C. 31. R

Gron. 
Gron. mus. I. n. 55. Idiem.

It. $\int \mathrm{c} a n .236 . t .2 . f .2$. GaD. $14,23,18 . P .16 . V \cdot 4$. A. 28,I9.C.dus Hvitling.

D. $14, \mathrm{I} 8,10.1 .20 . V \cdot 6 . A \cdot 30.20 . C . \ldots$

It. Wgot.176. Gadus Hvit-

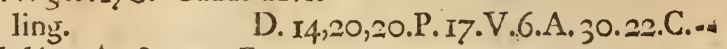
Habitat in Oceano Europaeo.

Carbona- 9. G. tripterygius imberbis, rius. maxilla inferiore longiore, linea laterali recta.

Art. geni. 20. (jn. 34. Gadus dorfo trip erygio imberbi, maxilla inferiore longiore linea laterali reata. D.I4,20,22.P.I8.V.6.A.22,19.C.a: Habitat in Oceano Europaeo.

Polla- IO. G. tripterygius imberbis, chius. m:xilla interiore long10re, linea laterali curus. Art. gent. 20. $(y z z .35 . \mathrm{G} 2=$ du dorfo tripterygy imberbı, maxilla interiore longiore, linea laterali curua. D.II, I9, 16.P.I7.V.6.A.I6, I8.C... Gron. muj: 1.n.57. Idem.

It. Wgot. 177. Gadus Lyr-

$$
\text { D. } 13,17,23 \cdot P \cdot 17 \cdot V \cdot 6 \cdot A \cdot 24,2 j \cdot C . \cdots
$$
bjek గ. Zaj.

D. I3, I9, I8.P.I6.V. 6.A.27,I3. C. .Habitat in Occano Europaeo.

* * Pinnis dorjalibus duabus.

Merlue- II. G. dipterygius cirratus, cius. maxilla inferiore longiore.

Art. gen. 22. $\int y n .36 . \mathrm{G} 2-$

D.10, 39.P.12.V.7.A.38.C.22, dus dorio diprerygio, maxilla inferiore longiore. D.9,40.P.12.V.7.A.39.C. =

. Habitat in Ocenno.

Molüa. 22. G. dipterygius cirratus, maxilla lif eriore lono giore. 


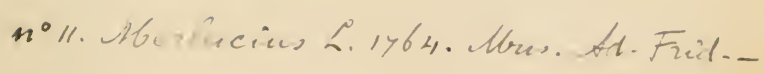



Art. gen.22. $\int y n \cdot 36 . \mathrm{Ga} \cdot$ dus dorfo divterygio, ore cirrato, maxilla fuperiore longiore.

It. Wgotb.177. Gadus Ion-

D. 15, 65. P.I5. V. C.A. 62. C... gus.

D. $15,63 \cdot P \cdot 20 \cdot V \cdot 6 \cdot A \cdot 60 . C . \cdots$

Habitat in Oceano Europaeo.

Lotav 13. G. dipterygius cirratus, maxillis aequalibus, $F$ z firec. 292.

Airt.gen. 22. Jyn. 38. Gadus dorfo dipterygio, ore cirrato, maxillis aequalibus. D.13,76.P.21. V.7.A.55. C. .Art. Jêp. 107. Silurus cirro in mento vnico. D. $13.76 . P .21 . V .7 \cdot A .55 \cdot C .30$. Habitat in lacubus Europaeis.

Muftela. I4. G. dipterygius cirratus, cirris 5 , pinna dorfuli priore exoleta.

Minf: Ad. Fr. I. p.75. Gadus dorfo dipterygio, cirris maxillae fuperioris 4 , inferioris $\mathrm{I}$.

Art. gen. 22. $\int y n .37$. G.

D.1,42.P.I4.V.7.A. 40.C... dorfo dipterygio, fulco ad pinnam dorli priman, ore cirrato.

Gron. AC7. Vpj: 17 42.p.93.

D. $1,56 . P .-. V .7 \cdot A .47 \cdot C . \cdots$

t. 3. Idem. B.7.D.I,4S.P.16.V.7.A.43.C.25. Habitat in Oceano Europaeo.

* * * Pinna dorfali unica.

mediter- 15. G. monopterygius cirra.

saneus. tus, maxilla fuperiore cir. ris duobus, inferiore vnico. D. 54 . P. I5. V.3. A. 44. C... Habitai in Ocenno Europseo. 


\section{PISCES IVGVLARES. Blennius.}

130. BLENNIVS. Caput decline, tectum. Membr. branch. rad. VI.

Corpus lanceolatum. Pinnae ventrales didactylae, muticae. Pinna ani diftincta.

* Caput criftatunn.

Galerita. I. D. crifta capitis transuerfa curacea. Art. gen. 27. fyn. 4.4 .

Habitat in Oceano Europaeo.

criftatus. 2. B. crifta fetacea longitudinali inter oculos. Gron. mil.I. 2.75 B.5.D.26.P.I4.V.3.A.16.C.11. Habitat in Indiis.

cornutus. 3. B. radio fimplici fupra $\mathrm{o}$ culos, pinna dorfali folitaria. Muf.Ad. Fr.2. p.. D.33.P.15.V.2. A.23.C. 12. Amoen. acad. I. p.3I6. Blennius pinnis ocularibus fubulatis, pinna ani officulorum XXVI. D.34.P.15.V.2.A.25.C. II. Habitat in India.

ocellaris. 4. B. radio fimplici fupra $\mathrm{O}_{-}$ culos, pinna dotfali anteriore ocello ornata. $M u f$. Ad. Fr. 2. p. D. II, I5. P. I2. V. 2. A. I6. C. II. Art.gen. 26. Jyn. 44. Blennius fulco inter oculos, macula magna in pinna dorrali.

D. .-P.12.V.2. A.17.C.26。 Habitat in Europa.

Gattoru- 5. B. pinnulis fuperciliorum gine. nucinaeque nalmatis. D.30.P.I3.V.2.A.2I. C.I2. Muf' 4d.Fr. I. t. 68. Blennit vertice fupercilisoque ciliato criffatis. D. $\frac{18}{30}$ P. I4. V.2. A. 20. C. 13. Art. 


Ait.gen. 26. Jyn. 44. Blennius pinnulis 2 . ad oculos, pinna ani officulorum XXIII.

Habitat in Europa.

D. $\frac{2}{31} \cdot P \cdot I 4 \cdot V \cdot 2 \cdot A \cdot 23 \cdot C \cdot 12$.

fuperci- 6. B. pinnulis fuperciliorum liofus. palmaris, linea laterali curua.

Amoen. acad. I. p. 317. Blennius pinnulis ocularibus breuiffimis palmatis, linea laterali curua. D. $\frac{34}{40}$ P.12. V.2. A.26. C.12. Gron. mul: 2. n. 172. t. 5 . $f$. 5. Blennius officulis anterioribus pinnae dorfalis maioribus. D. $\frac{36}{44} \cdot$ P. I5. V.2.A.28. C. 12.

Habitat in India.

* * Caput alsque criftis.

Pholis. 7. B. capite laeui, linea laterali curua fubbifida. Art. gen. 27. Jyn. 44. (ct D. $\frac{1}{2} \frac{2}{8} \cdot P \cdot x 4 \cdot V \cdot 2 \cdot A \cdot 19 \cdot C \cdot 10$. Plolis) I16. Blennius capite lummo acuminato, maxilla fuperiore longiore.

D. 36. P. .- V.2. A.28. C. .Gron.muf.2.n.175. idem. B.6.1. $\frac{1}{3}$. P.I3. V.2. A.19. C. 10. Habitat in Oceano et Mari Mediterraneo.

Gunnel- S. B. pinna dorfali ocellis $\mathrm{X}$ lus. nigris. Muj: Ad. Fr. 1 . p 69. D.77.P. I0. V.2.A.43. C. I6. Art. gen. 27. $\int y n \cdot 45$. Blennius maculis circiter $\mathrm{X}$ nigris, limbo albo, verinque ad pinnam dorfalem. D.78.P.II. V.2. A. $\frac{2}{40} \cdot$ C. -Habitat in Oceano Atlantico.

muftela- 9. B. pinna dorfali anteriore ris. triradiata. Muj: Ad.Ir.
I. p. 6y.
D.3,43.I.17. V.2.A.29.C.13. 
8. B. pinna dorfi anteriore radiis 3 , pofteriore 40. Muf. Ad.Fr. I.p.69. Hobitat in India.

D.3,40.P.I6.V.2.A.28.C.I2.

viuipa- 10. B. ore tentaculis duobus.

rus. Ait. fyn. 45. Blennius capite dorloque fufco-flauefcente lituris nigris, pinna ani flaus.

Mre: Ad. Fr. x. p. 69 . idem. D.80.P.19.V.2.A.66.C... Gron2. muf. x. p. 65. idem. D...P.19. V.2. A. .. C. .. Gronr. Act.VPS. I742. p. 87. idem.

D. - P. Ig. V.2. A. - - C. -ACt. Stockh. 1748.p.32. t.2.

Tinglake.

Ilabitat in M. Europaeo.

Viuos parit foetus, quod fungulare.

Lumpe- II. B. corpore areolis dorfanus. libus fufcis. Art. Syn. 45. Blennius cirris fub gula pinniformibus quali bifidis, areolis dorfi transuerfis.

Habitat in Europa.

taninus. 12. B. pinnis ventralibus fubfexfidis, cirro gulari. D.66.P.22.V. $\frac{2}{6} \cdot A \cdot 60 \cdot$ C. 30 . Habitat in Sueciae lacubus.

Cum radii pinnarum ventralium quidem 6 , fed 2 primo. res in vtraque fetacei fint, et tamquam veri babendi, poterit forte pifcis ad boc genus referri, ob babitunn cum duobus praecedentibus. 



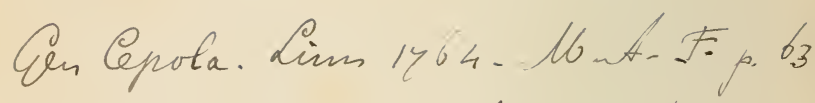

Cepota rubescens o. 1ybu. M. A.F. 10.63

Cepola pimea caubae attenuato, -maxillis cutis Tacmia terpens rubescens dicta Art. fyr 115
ibab: deediters.

Capnt nodum conpresfum, maxill's acntis, dentatis diembr. Granchiost. rasios 6.

Corpus terpentis, valde compresfur, abaque spinis et squamis D $68 P_{17} V 6$ As C B 
I3r. OPHIDION. Caput nudiufculum.

Menbr. branch. patula radiis V. Corpus enfiforme. Pinna dorfallis anique vnita catidae. Pinnae ventiales radiis duobus: exteriore fipinofo.

barba- $\quad$. O. maxilla fuperiore cir-

tum. ris quatuor. Art.gent. 25. fyn. 42.

Habitat in Europa aufirali.

âmberbe. 2. 0 . maxillis imberbibus, cauda obtufiufcula.

Art. gen. 25. Jyn. 42. O. phidion cirris carens.

Fin. fiec. 289. Idem nomen.

D.79. P.1I. V.2. A. 4I. C. I8:

Mabitat in Europa.

macroph- 3. O. maxillis imherbibus,

thalmum. pinna caudae acuminata. D.69.P.15. V.6.A.62.C.1 $\Rightarrow$ Habitat in M. Mediterraneo. E. Brander. 
260 PISCES THORACICI. Cyclopterus.

\section{TH O R A C I C I.}

132. CY CL OPTER VS. Caput obtufum. Maxillae denticulatae.

Membr.branch. radiis IV.

Corpus fquamis offeis diftantibus angulatum. Pinnae ventrales connatae in rotatam.

Lumpus. I. C. corpore fquamis offeis angulato. Muf. Ad. Fr.

I. $p .57$.

Frl. fiec. 275 . It. fcan. IS8. Cyclopterus. D.0:2I.P.20.V.6.A.10.C.g. Art. gen. 62. Jyn.87. Cycloprerus.

Gron. muґ.n. 127. Cyclopterus.

D.21.P. 20.V.15. A. 10.C. 12. Habitat in Oceano Balthico et feptentrionali. Affinitas Jumnna cun cartilaginojo Acipenfire.

nudus. 2. C. corpore nudo. $M u f \cdot A d$.

Fr. I. p.57.t.27.f.I. $\quad$ B. I.D.6.P.2I.V.4.A. -- C. 10. Habitat in India.

133. ECHENEIS. Caput pingue, nudum, depresfum: fupra planum, marginatum, transuerfe fulcato - ferratum.

Menubr.branch. radiis X.

Corpizs nudum.

Remora. I. E. cauda bifurca, ftriis ca-

pitis I8.

Annoen. acad. I. p.320. E-

cheneis.

Art. gen. I5. Jin. 28. Eche-

D. 22. P.28. V.5. A. 22. C. 16 . neis. 




\section{PISCES THORACICI. Echeneis.}

Gron. mul: I. 33. Echeneis.

D.22.P.25.V.6.A.22.C.I7.

Catesh.carol. 2. t. 26. Remora.

Habitat in Pelago Indico.

Cupitis clypeo adbaeret aliis.

Neucra- 2. E. cauda integra, ftriis

tes.

çapitis 24.

D. $37 \cdot$ P.21. V.5.A. $37 \cdot C .16$.

Vallifin. nat. I. t. 44 .

Haplelqu. itin. 324. n. 68 .

Echeneis Naucrates.

Habitat in Pelago Indico.

Differt a pracedenti cauda longiore, corpore morore, pinnis acutioribus.

134. CORYPHAENA. Caput maxime truncato-decliue.

Membr. branch. radiis V. Corpus: Pinna dorfalis longitudine Dorfi.

Hippu- I. C. cauda bifida, radiis dor-

rus. falibus LX. Loejl. epifl. D.60.P.2r.V.6. A.26.C. Is. Osbeck itin. 307 . B.IO.D.60.P.19.V.6.A.27.C. 20. Art. gen. 15. Jjn. 29. Co-

ryphaena cauda bifida. D...P. 20. V. 6.A... C. I8. Habitat in Pelago; Nautis Dorado dicfus a colore Jplendidiffinno.

Equife- 2. C. cauda bifurca, radiis

lis. dortalibus LIII.

Osb. itin. 308. Dorado. B.6.D.53.P.r9.V.6.A.23.C.20。

Marcgr. braj: 16o. Guara-

capema.

Habitut in alto Pelago.

Pulcberimus, a priori parum diftingus.

pentada- 3. C. maculis nigris quinis Etyla. verfus caput longitudinalibus.

Ait. Stockb. 1740. p. 460.

t. 3. $f .2$. Elennius ma-

R ; 
culis 5 vtrinque verfus

caput nigris.

Valent. amb. 5. p. 435. $t$.

C. $4 \cdot$ D. $\frac{9}{21} \cdot$ P.II.V. $\frac{1}{6} \cdot A \cdot \frac{3}{14} \cdot$ C. I 3

292. Rievier Dolfyn.

IVill. icbs. app.7.t. 8.f. 2.

Habitat in India.

Nouacu- 4. C. capite pinnisque can-

la. cellatis lineis caerulefcentibus.

Art. gen. 15 fyn. 29. Corypinaena palmaris puichre varia, dorfo acuto.

Ilabitat in M. Mediterraneo.

Pompi- 5. C. dorfo fupra lineam lalus. teralem curuam fafciolis flauefcentibus picto. B.5.D. $\frac{8}{33} \cdot$ P.I4.V.6.A. $\frac{2}{14} \cdot C \cdot 16$. Avt. gen. I6. Syn. 29. Coryphaena cauda aequali,linea laterali curua.

Habitat in Pelago.

Corpus pingue, pofice attenuatum. Linea lateralis curua, flavefcens. Caput obtufim. Maxilla inferior adfcendens. Os valde debijcens, maxillis latere cabaerentibus, intus fcabrum. Capitis latera cauernoja dentata. Pinna dorfalis prior vnita pofteriori. Pinna pectorales admodum acuminatae.

135. GOBIUS. Caput poris 2 inter oculos approximatos: altero anteriore. Membr. branch. radiis IV.

Pinnae ventrales vnitae in ouatam I 2 - radiatam.

niger. I. G. pinna dorfi fecunda radiis quatuordecim. Art. gen. 28. Syn. 46. Gobius e nigricante varius, pinna dorfi fecunda officulorum I4. Mul: Ad. Fr. I. p. 74. idem.

D.6,I4.P. I8.V.IO.A. II.C. I4. D.6,I4. P.I7. V..-A.I4. C. $\approx$ Osbeck. 

Gozo 21764-Mus. Ad. Frix. po. 65 
Osbeck. iter. I3I. Apocryptes cantonenfis.

Habitat in Europa, Afia.

D. 6, I4. P.10.V.I2. A.13.C.18.

Paganel- 2. G. pinna caudae dorfali-

lus. que fecunda bafi purpura. fcente, priori linea lutea terminali.

Art. gen. 29 fyn. 46. Gobius linca lutea transuer$\mathrm{fu}$, in fummo pinnae dorfilis primae.

Haffelqu. itin. 326. Gobius P.aganellus.

Habitat in Mari Mediterraneo.

D. 6,I7.P.I7.V.I2.A.I6. C.20.

Eleotris. 3. G. pinna ani radiis nouem.

Chin. Lagerftr. 28. B. 5.D. 6,Ir.P. 20. V, I0.A. 9.C.10. Osbeck. itcr.260. Gobius chinenfis.

B.5.D.6, II.P. Is. V.8. A.S.C. I2.

Amoen.acad. I.p.3II. Trachinus capite inermi nudo, pinnis pectoralibus coadunatis.

Cron. mil:2.13.169. Eleo-

D. 6,IO.P.I6. V.I2.A.IO.C.I4. tris pinnis ventralibus concretis?

Habitat in China.

D.6, I2.P...V.I0. A. 9.C...

Aphya. 4. G. fifciis etiam pinnarum fufcis.

Art. Gen. 29. Jym. 49. Go-

D. 6, I6.P.IS. V.I2.A.I4. C.I3. bius vncialis, pinna dorfi

fecunda officulorum 17 . D.6, 17.P.17. V.6. A.11. C.-Hubitat in Nilo.

\%oro

5. G. radiis dorfalibus eminentibus fetaccis.

Art. gen. 29. Jyn. 47. Gobius pinna ventrali cacrulea, officulis pinnae dorfalis fupra membranam a!lurgentilus.

IIabitat in Europa aiflrali. 
pectini- 6. G. dentibus maxillae infe-

roltris. rioris horizontalibus.

Chin. Lagerftr. 29. f.3. D.5,26. P.r9. V.12. A.25.C.15.

Osúeck. iter. 130. Apocrypres chinenfis. B. 5.D.5, 26.P.18.V.IO. A.26.C... Habitat in China.

anguilla- 7. G. pinna dorfali vnica. D.52.P.I2. V. Io. A. 43. C. I2. ris. Hubitat in China.

Corpus lubricum Anguillae pingue, fubdiapbana cute vt venae transpareant. Pinnae omnes rubrae: Dorfaliset Ani in caudan cxcurrentes. Os fomum dentibus exjertum. Pinnae pectorales valde paruae, rotundatae.

136. COTTVS. Caput corpore latius fpinofum. Membr. branch. radiis VI. Corpus...

cataphra- I. C. loricatus; roftro verहtus. rucis 2 bifidis, capite fubtus cirrofo.

Art. gen. 49. $\int y n \cdot 77 \cdot \int p e c$.

87. Cottus cirris pluri-

mis, corpore octagono. D. $\frac{5}{12}$. P. I5. V. $\frac{1}{3}$. A.6.C.II. Muf. Ad. Fr. I.p.70. idem. D.5,7. P.16. V.2. A.7.C.II. Habitat in M. Europaeo.

quadri- 2. C. verrucis capitis 4 ofcornis. feis. Fin. Juec. 278. Syft. nat. 6. p. 47. t. 4. $f \cdot 3$. Muf. Ad. Fr. . p. po. $t$. 32. $f .4$.

Art. gen. 48. Jpec. 84. Cottus faber, tuberculis 4 corniformibus in medio capite.
D. 9,I4.P.I7.V. 4.A.I4.C.I2.

Habitat in M. Balthico.

grun- 3. C. gula ramentis villofa, niens, corpore nudo. Muf: Ad.

Fr. 2. p.
Varietas alia, $\longrightarrow$ D.2,26.P.I8.V. $\frac{\text { I }}{2}$ A.22.C.I2.

Gron. 


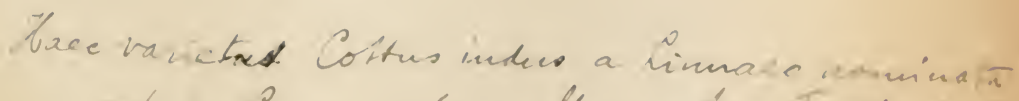

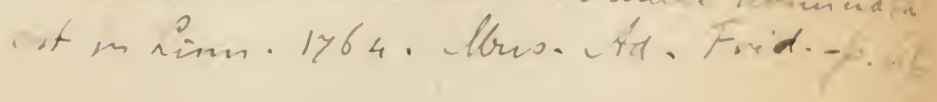



Gron. mi!. I.n. 106. Cotrus alepidotus varius, maxilla inferiore longiore multum cirrata.

Marcgr. braj: 178. Nigui. D. $\frac{2}{25} \cdot P \cdot 23 \cdot V \cdot 3 \cdot A \cdot 19 \cdot C \cdot 15 \cdot$ Will. iclot. app.t. $4 . f . \mathrm{I}$. Gallus grunniens.

Habitat in America.

fcaber. 4. C. capite ftriis corporisque iquamis ferratis, linea laterali eleuata. Habitat . .

B.7.D. 9, II.P.2.V. $\frac{1}{6}$.A.II.C.r2.

Scorpius. 5. C. capite fpinis pluribus, maxilla fuperiore paulo longiore.

1t. jcan. 325. Vlka. D.9,16.P.16.V.3.A.12. C.I2. Art. gुen. 49. fyn. 77. Spec. 86. Fn. fiue 280. Cottus alepidotus, capite polyacantho, maxilla fuperinre paulo longiore. D.7, 17.P.14. V.4. A.13.C.8. Muf. All. Fr. I. p. 70. Cot.
tus idem.
D. 10,14 .

Gron. muj"I. n. 104. idem. D.I0,I4.P.16. V.4.A.10.C.I2. Habitat in Oceano Europaeo.

Gobio. 6. C. laeuis, capite fpinis duabus.

A.t. gen. 4S. Jyn. 76. Spec. 82. Cottus alepidotus glaber, capite diacantho.

In. fiuec.279, idem.

Gron. mul: 2. 12. 166. VraD. $7,17$. P.I4. V. 4. A.I3. C.8. D.7,IS.P.I4.V.4.A.I3. C.IO. nofecopus capite verinque monic inthin.

Habitut in Vurnpac furies.

D.7, IS.P.I4. V.4. A.12.C.13. Nidum in fundo format, ouis incubat. 
137. SCORPAENA. Caput magnum, aculeatum: Oculi vicini. Dentes ma. xillis, palato faucibusque. Membr. brancb. radiis VII. Corpus. .

P'arcus, I. S. cirri ad oculos naresque.

Muf.Ad.Fr. I. p.68. Zeus cirris fupra oculos et nares.

Art.gen. 47. Jyn. 75. Scor-

$$
\text { D. } \frac{12}{22} \cdot P \cdot 18 \cdot V \cdot 7 \cdot A \cdot \frac{3}{8} \cdot \text { C. } 15 \text { a }
$$
paena pinnulis ad.oculos

et nares.

D. $\frac{12}{2 I} \cdot P \cdot 18 \cdot V \cdot \frac{\pi}{6} \cdot A \cdot \frac{3}{8} \cdot C \cdot 12$. D. $\frac{2}{2} \frac{2}{1} \cdot$ P.I6. V. $\frac{x}{6}$. A. $\frac{3}{8}$. C. . Hajfelqu.itin.330. idem. D. $\frac{12}{2}$. P.16.V.6.A. $\frac{3}{8}$. C.13. Habitat in M. Mediterraneo, Óceano.

Scrofi. 2. S. cirris' ad labium inferius. Art.gen. 47. Jyn.76. Scorpaena tota rubens, cirris plurimis ad os. $\quad$ D. .. P. I9. V... A. . C C. - -

- Gron. muf. r. 21.203. Scorpaena capite cauernofo, cirris geminis in maxilla inferiore?

Habitat in M. Nediterraneo,

D. $\frac{1}{2} \frac{2}{2} \cdot P \cdot I j \cdot V \cdot \ldots A \cdot 6 \cdot C \cdot 13$ :

38. ZEVS. Caput comprefium, decline: Lab. fuperius membrana transuerfa fornicata. Lingua fubulata.

Membr. branch. radiis VII perpendi: cularibus: infimo transuerfo, Corpus compreffum.

Vomer. I. Z. cauda bifurca, fpina ante pinnam ani decum. bente.

Brown. iam. 454. Rhom boida aiepidota argentea, pinnis omnibus breuibus. 

Mif. Ad. Fr. I. p. 66. t. 3. $f$.. . Zeus cauda bifurca.

Habitat in America.

D.8. $\frac{\mathrm{I}}{22} \cdot \mathrm{P} \cdot \mathrm{I8} \cdot \mathrm{V} \cdot 4 \cdot \mathrm{A} \cdot \frac{\mathrm{I}}{\mathrm{I} 9} \cdot \mathrm{C} \cdot 20$.

Color argenteus. Pinna dorfi antevior radiis $4:$ pofterioribus diftinetis brenifinis finofis; Jpinae 2. pone anum. Linea lateralis valde curua.

Gallus. 2. Z. radio dorfali decimo analique fecundo corpore lungioribus.

Arted.gen. 35.Jin.78. Zeus cauda bifurca.

Gron. muf. I. n.108.'idem D. $\frac{1}{3} \cdot$ P.20. V.6.A. $\frac{1}{20} \cdot$ C.I7. Brown. ian. 455 Rhomboida maior alepidota, radiis anterioribus pinnae dorfalis et ani lungiffmis. Marcgr: lraj: I6I. Abucatuia.

Ilalitat in America.

Faber. $\quad 3 . \mathbf{Z}$. cauda rotundata, lateribus mediis ocello fufco.D.IO,23.I.I3.V.I. $\frac{I}{6} \cdot A \cdot 4, \frac{4}{2}, C \cdot I 3 \cdot$ Art. ge11.50. Jyn 78. Zeus ventre aculeato, cauda in extremo circinata. D. IO, 24. P.I4. V. I. A. $\frac{4}{7} \cdot 6^{\circ}$ C. . Mus. Ad. Fr. I. p. 67.t.31. $f: 2$. Zeus ventre aculeato, cauda rotundata. D. 10,23.P.I3.V. I. A.2I.C.I5. Gron.mul:1. 12.107. idem. D.10,21.P.13.V.7.A.4, 22.C.14. Ilabitat in Pelago.

Aper. 4. 'Z. cauda aequali, corpore rubente.

Art. geil. j0. Jyn. 78. Zeus catida aevuali rotus rubens, roftro reflexo. D.9,23. P.I4.V. $\frac{1}{6} \cdot A \cdot \frac{3}{26} \cdot$ C. - a llabitat Romae, Cienuae. 
139. PLEVRONECTES.(*) Caput: Oculi ambo in eodem latere capitis. Membr. branch. radiis IV-VII.

Corpus compreffum, latere altero dorfum, altero abdomen referente.

* Oculis a latere dextro.

Achirus. I. P. oculis dextris, corpore glabro, pimis pectoralibus nullis. Gron. mul. I. n. 42.

D.60. P. ..V.4.A. 48. C. 16. Habitat Surinami.

Corpus brumenn lineis nigris transucrfis raris. Latus finiftrum capitis cirrofunn.

trichoda- 2. P. oculis dextris, corpore

Eylus. afpero, pirnis pectoralibus filiformibus.

Art. gen. 18. Jyn. 33. Spec.

D. 53 . P. 4 . V.5. A. 43. C. 16. 60. Pleuronectesafper canefcens, pinnis lateralibus vix conf́picuis.

Habitat in Amboina.

Corpus fufcunn, maculis obfcuris varium.

lineatus. 3. P. oculis dextris, lineis lateralibus feptem transuerfis.

Pleuroneetes fufcus fubro. tundus glaber, lineis feptem nigris transuerfalibus inter pinnam pectoralem et caudam. Browon. iam. $445^{\circ}$

Paffer

(*) Pleuronectes inter omnia animaiia fingularis ocalis ambobus ab eodem latere cafitis et corpore later' $\int u$ periore colorato, altero inferiore pallido, natatu obliquo. 


Paffer lineis transuerfis notatus. Sloan. iam.2. p. 277.t. 246. f.2. Rai, pifc. I57.

Habitat in America,

ocella. 4. P. oculis dextris, corpore od

tus. cellis 4 atris iridibus albis. D.66.P.3.V.6.A.55.C. I4 Habitat Surinami.

Pinna dorjalus plicata. Pinna caudae fajcia nigra.

lunatus. 5.! $\mathrm{P}$. oculis dexrris, punctis ocellisque dimidiarıs caeruleis fparfis.

Catesb. car.2. p.27.t.27. Solea lunata et punctata.

Habitat in America Jeptentrionali.

Hippo- 6. P. oculis dextris, corpore glofius. toto glabro. Fn. Jivec. 302 . Art. gen. 17. Jyn. 31. Pleuronectes oculis a dextra totus glaber. $\quad$ D. 105. P.15. V.6.A.79. C... Gron. muj:2.n.158.idem. D.102.P.16.V.6.A.78.C.I9, Habitat in Oceano Europaeo.

Cyno- 7. P. oculis dextris, corpore glolius, oblongo glabro, dentibus obtulis, cauda fubrotunda. Gron. muj: 1. n.39. D. II2.P.11.V.6.A.102.C.24. Habitat in Oceano belgium alluente.

Plateffa. 8. P. oculis dextris, corpore glabro, tuberculis 6 capitis. In. Suec. 301 .

Art. gen. 17. Jyn. 30. Pleuronectes oculis et tuberculis 6 in dextra capitis, lateribus glabris, fina ad anum.

1t. Wgot. 179. nomen In. fuec.

It. Jcan. 326. Pleuroneles

$$
\text { D. } 77 . P .12 . V .6 . A .55 . C . . .
$$
D.74.P.II:6.V.6.A.54.C. 20 , Slatvar. D.76. P.12. V.6.A. 54.C.17. 
Gron. musf: 1. n. 36. nom. Arted.

Habitat in M. Europaeo.

Flefus. 9. P. oculis dextris, linea laterali afpera, fpinulis ad pinnas. Fn. $\int u .300$. D.55. P.II. V.6.A. 40.C.I4. Art. gen. 17. Jyn. 33. Pleuronectes oculis a dextris, linea laterali afpera, fpinulis fuperne ad radices pinnarum, dentibus obtufis.

Avt. Jpec. 59. Pleuronectes linea laterali afpera, ipinulis ad radices pinnarum in latere oculato.

D.61.P.12.V.6.A. 42.C.19. Gron.mul: I. n.40. idem. D. 62.P.12.V.6.A.43. C. I4. It. jcan. 326. idem. Habitat in $M$. Europaeo. D. 54.P.11:10.V.6.A.41.C.17.

Limanda. 10. P. oculis dextris, fquamis afperis, fpina ad anum, dentibus obtufis. Art. gen. 17. Syn. 33. $\quad$ D.79. P. 12. V.6.A.60. C... Habitat in M. Europaeo.

Solea. II. P. oculis dextris, corpore afpero oblongo, maxilla fuperiore longiore. Avt. gen. I8. Jyn. 32. Jpec. 60. Pleuronectes maxilla fuperiore longiore, corpore oblongo, fquamis vtrinque afperis.

Fn. Juec. 299. idem.

It. Wgot. 178. Pleuronectes Tunga.

Habitat in M. Europaeo. D. 9I. P. 9. V.5.A.74. C.I4. D.71.P. 8:7.V.5.A. 40.C. 40.

Lingua- 12. P. oculis dextris, ano fitula. niftro, dentibus acutis. Art. gen. 17. Jyn. 31. Pleuronectes oculis a dextra, 


ano ad latus finiftrum, dentibus acutis. $\quad$ D. 65. P. 9. V... A. 45. C. . Grun. muf:1.n. 41. idem, D.68.P.10. V.6. A. 55. C. I9. Habitat in M. Europaeo.

* Oculis a latere finiftró.

Rhom- 13. P. oculis finifris, cotbus. pore glabro. Art. gen. 18. Jyn. 31. $\quad$ D.76.P. I0. V. 6. A. 57 . C.-It. Wgoth. 178.Pleuronectes Pi.gghvarf. Gron. mul: I. n. 43. nom. Arred. D.79. P.10. V.6.A.56.C.16. Habitat in Oceano Europaeo.

Ma ximus Domitiani tempore cattus, snde Senatus confult. Ex boc tempore, iam Caejar figuli tua caftra jequantur.

maximus. I4. P. oculis finiftris, corpore afpero. Art. gen. I8. jyn. 32. Fn. juec. 298 . idem. D.64. P. II. V.6. A. 48. C. I6. It. Gotbl. 178. 1dem. D. D9. P.12. V.6. A 39. C.I6. Gron. mul.2. n.159.iden. D. 65. P.13. V.6.A. 50. C.16. Habitat in Oceano Europaeo.

Paffer. 15. P. oculis finiftris, linea laterali liniftra aculeata. Art. gen. 1S. Jyn. 32. Pleuronectes oculisa liniftra, linea laterali aculeata. D.66.P.9. V.6. A.50.C... Habitat in Oceano Europaeo.

papillo- 16. P. oculisfiniftris, linea lafus. terali curua, corpore papillofo. Marcgr. braf. ISI. Aramaca. D. 58.P.12.V.5:6.A.42.C.16. Habitut in America. 
140. CHAETODON.(*) Caput: Dentes fetacei, flexiles, confertiflimi, numerofifini.

Membr. branch. radiis VI. Corfus pictum. Pima dorfi anique carnofa fquamofa.

cane-

fcens.

I. C. fpinis dorfalibus 2, 12dio tertio longiflimo filiformi, aculeo vtrinque ad os, cauda bifida. Art. Jpec. 93. Chaetodoncanefcens, aculeo vtrinque ad os, officulo tertio pinnae dorfalis fetiformi longiffimo. D. $\frac{2}{46} \cdot P \cdot 17 \cdot V \cdot \frac{1}{6} \cdot A \cdot \frac{5}{36} \cdot C \cdot 16$ Habitat, in Indiis.

argentE:IS.

2. C. Fpinis dorfalibus 3 , cauda bifida, pinnis ventralibus ex fpinis duabus. Chin. Lagerftr. 26.

D. $\frac{3}{32} \cdot$ P. 26.V. $\frac{2}{2} \cdot A \cdot \frac{3}{3} \cdot$ C.17. Habitat in Indiis.

acumina- 3. C. Ppinis dorfalibus 3: ratus. dio tertio longiffimo. Muf. Ad. Hr. I. p. 63.t. 33. f. 3. Chaetodon fafciis 3 fufcis, pinna dorfali fetiformi.

D. $\frac{3}{28} \cdot$ P. I6.V. V. $\cdot A \cdot \frac{3}{19} \cdot C \cdot 17 \cdot$

Habitat in Indiis.

pinnatus. 4. C. fpinis dorfalibus 4, pinna dorfali analique long: flima.

Muv. Ad. Fr.p. 64. t. 33.

f. 6. Chaerodon grifeus,

fafcia frontali apiceque

$\begin{array}{ll}\text { caudae albis. } & \text { D. } \frac{4}{40} \cdot \text { P.IS.V. } \frac{T}{6} \cdot \text { A.2S. C. IS. }\end{array}$

Clin.

(*) Chaetocion Caudae pinna bifida, $7,2,5,20,21,74,28$.

Rofiro cylindrico elongato, $2,22,16$.

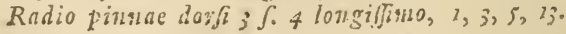




Chin. Lagerftr. 25. idem. D.30. P. I8.V. I. A.28. C.I8. Habitat in Indiis.

cotnutus. 5. C. fpinis pinnae dorfalis 7 , cauda bifida, radio dorfali tertio longiffimo.

Ait. Jyn. 92. Chaetodon aculeis 2 breuibus fupra oculos, officulo tertio pinnae dorfalis longifir. mo.

Habitat in Indiis.

$$
\text { D. } \frac{7}{46} \cdot P \cdot I 8 \cdot V \cdot \frac{1}{6} \cdot A \cdot \frac{3}{36} \cdot C .16 .
$$

puncta- 6. C. fpinis pinnae dorfalis $S$, tus. pinnis peetoralibus falcatis. $\quad$ B.4. D. $\frac{8}{30} \cdot$ P.I7. V. T.A. $\frac{3}{20} \cdot$ C. I7. Habitat in Afra. Mul: Ac. Holmenl:

Corpus albidum $f$. argenteun punctis fulcis, pone anum dilatatum, figura Cyprini. Oculi magni, rubri. Linea lateralis jiw jum curua. Radii 3 primi p. ani diftantes.

arcuatus. 7. C. fpinis pinnae dorfalis 8 , arcubus 4 albis.

Mu!: Ad. I\%. I. p. Gr. t. 33 . $f .5$. Chaetodon fufcus, arcubus 5 nigris. D. $\frac{8}{3} \cdot 1$. $.20 \cdot V \cdot \frac{T}{5} \cdot A \cdot \frac{3}{27} \cdot C \cdot$ I9. Art. Syn.79. Spec. gr. Chaetodon niger, capite diacantho, lineis verinque 4 transuerlis curuis.

Habitat in Indiis.

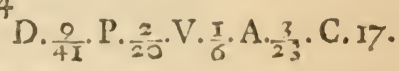

roftratus. 8. C. fpinis pinnae dorfalis 9 , maculaque ocellari, roftro cylindrico.

Muj: Ad. Fi. r. p. 6r. $t$. 3I. $f$. 2. Chactodon roftratus pinna dorfali pofice macula fufca. D. $\frac{2}{40} \cdot P \cdot I_{4} \cdot V \cdot \frac{x}{6} \cdot A \cdot \frac{3}{3} . C \cdot I_{4}$.

Gron. muf. r. n. 109. Chaetodon mecrolepidorus albo-flauefens, roltro lon$\mathrm{S}_{3}$ 
gifimo offeo, macula nigra ad pinnam dorfalem. D, $\frac{9}{39} \cdot P, 15, V \cdot \frac{\pi}{6}, A \cdot \frac{3}{24}, C . \cdots$ Habitat in Indiis.

nigricans. 9. C, fpinis pinnae dorfalis 9, fpina laterali virinque caudae integrae.

Art. fiec go. Chaetodon nigrefcens, cuuda albefcente aequali vtrinque acu. leata,

IHafelqu, itin. 332. ChaetoD. $\frac{9}{3 \frac{8}{8}} \cdot$ P.16. V. $\frac{1}{6} \cdot A \cdot \frac{3}{29} \cdot C \cdot 16$. don aculeis in vtroque latere ad caudam duobus, D. $\frac{6}{3} \cdot \mathrm{P} \cdot 17 \cdot \mathrm{V} \cdot 6 \cdot \mathrm{A} \cdot \frac{2}{2} \cdot \mathrm{C} \cdot 2 \mathrm{G}$. Habitat in Mari rubro.

tiofs- 10. C. finis pinnae dorfalis इนร. 9 , membranae branchioftegae 3 , fatciis 5 nigris, caudu fubtifida.

Habitat in Indiis.

lincatus. II. C. fpinis pinnae dorfalis

9, fpuna latẹali vtrinque caud e bifidae.

Art. Spec. 89. Chaetodon lineis longitudinalibus varius, cauda bifurca vtrinque aculeata.

Habitat in Indiis.

D. $\frac{9}{3} \cdot P \cdot \frac{I}{5} \cdot V \cdot \frac{\pi}{5} \cdot A \cdot \frac{2}{22} \cdot C \cdot I 6$. D. $\frac{9}{36}, P \cdot 16 \cdot V \cdot \frac{x}{6} \cdot A \cdot \frac{3}{29} \cdot C \cdot 16$.

macrole- 12. C. fpinis pinnae dorfalis, pidotus.

II, radio dorfali quarto filiformi longiffimo.

Art. Jpec. 94. Chaetodon lineis virinque 2 nigris, radio quarto dorfali longiflimo fetiformi.

Gron. mus 2. n. 194. Ch. D. $\frac{1}{37} \cdot P, \frac{2}{18} \cdot V \cdot \frac{I}{6} \cdot A \cdot \frac{3}{2} \frac{3}{3} \cdot C \cdot I 7$. officulis dorfalibus 3 aculeatis, quarto fetiformi, 7 fubfequentibus iterum aculearis.

Habitat in Indiis, D. $\frac{11}{37} \cdot P, 19 \cdot V \cdot \frac{T}{6} \cdot A \cdot \frac{2}{2} \cdot C \cdot 16$ 




\section{PISCES THORACICI. Chaetodon.}

friatus. I3. C. fpinis pinnae dorfalis 12, corpore ftriato, roftro prominente, cauda integra. Mul: Ad. Fr. I. p. 62. t. 33 . f.7.Chaetodon flauefcens, fafciis 5 fufcis.

Art. Jpec 95. Chaetodon

D. $\frac{10}{30} \cdot P \cdot 15 \cdot V \cdot \frac{1}{6} \cdot A \cdot \frac{3}{21} \cdot C \cdot 13 \cdot$ macrolepidotus, lineis vtrinque tribus nigris latis, linea quarta in cauda.

Anoen. acad. I.; $p$. 313. Labrus roftro reflexo, fafciis lateralibus 3 fufcis. D. $\frac{1}{3} \frac{2}{3} \cdot$ P.14.V. $\frac{1}{6} \cdot A \cdot \frac{3}{20} \cdot$ C.I7. Gron. muf. I. n. IIO. Chaetodon roftro longo offeo macrolepidotus albo flauefcens lineis transuerfalibus 4 brunneis. Habitat in Indiis.

D. $\frac{1}{3} \frac{2}{3} \cdot$ P.I6.V. $\frac{1}{6} \cdot A \cdot \frac{3}{\frac{3}{1}} \cdot$ C.I\%. D. $\frac{1}{3} \cdot$ P.I5.V. $\frac{\pi}{6} \cdot A \cdot \frac{3}{21} \cdot C \cdot=-$

aruanus. I4. C. fpinis pinnae dorfalis I2, cauda bifida, fafciis 3 fuccis.

Muf: Ad. Fr. I. p. 63. t. 33 . $f$. 8 . Chaetodon cauda bifurca, fafciis 3 tufcis. D. $\frac{\pi}{3} \frac{2}{2} \cdot P \cdot I S \cdot V \cdot r \cdot A \cdot \approx=C \cdot I G$. Habitat in Indiis. Frons alba eft.

capiftra- 15. C. fpinis pinnae dorfalis tus. I2, corpore friato, ocelIn fubcaudali.

Mul: Ad. Ir. I. p. 63. $t$. 33. f. 4. Chaetodion pallidus, falcia capitis ocelloque fubcaudali fufcis. D. $\frac{7}{3} \frac{z}{2} \cdot P \cdot I 4 \cdot V \cdot \frac{T}{6} \cdot A \cdot \frac{3}{2 I} \cdot C \cdot 13$.

Gron. mul: 2. n. 195. Chaet. mucrolepidotus, linea nigra ad oculos, macula rotunda ad pinnam dorfalem.

Amuen. acad. 1. p. 3I4 $\mathrm{La}-$ D. $\frac{I}{30}=$ P...V. $\frac{x}{6} \cdot A \cdot \frac{2}{18} \cdot C .$. hrus toltro reflexo, ocello 
purpureo iride alba iuxta caudam.

Habitat in Indiis,

$$
\text { D. } \frac{13}{3} \cdot P \cdot I 4 \cdot V \cdot \frac{3}{6} \cdot A \cdot \frac{3}{17}, C \cdot \cdots
$$

vagabun- 16, C. fpinis pinnae dorfalis

dus. $\quad I_{3}$, corpoie ftriato, roftro cylindrico.

Mief. Ad. Fr. 2. p... Chae-

D. $\frac{13}{40} \cdot P \cdot 15 \cdot V \cdot \frac{1}{6} \cdot A, 24 \cdot C \cdot I 8$. todon roftratus, fafcia nigra trans oculos, triplicique ad caudam.

Hab tat in Indiis.

ciliaris. I7. C. fpinis pinnae dorfalis $I_{4}$, operculis fpinofis, fquamis ciliatis.

Gron. mul. 2. n. I92. Ch. macrolepidotus, aculeis vtrinque 2 in operculis, poftico longifímo.

Mul: Ad. Fr. I. p. 62. t. D. $\frac{14}{3} \cdot P \cdot 26 \cdot V \cdot \frac{\pi}{6} \cdot A \cdot \frac{3}{26} \cdot C \cdot I 7 \cdot$ 33. $f$. I. Chaetodon gुrifeus fafciis 4 fufcis.

Usb.iter. 273. Sparus faxatilis,

Habitat in Indiis.
D. $\frac{\pi}{3}+\mathrm{P} \cdot 20 \cdot \mathrm{V} \cdot \frac{\mathrm{T}}{6} \cdot \mathrm{A} \cdot \frac{3}{2} \frac{3}{3} \cdot \mathrm{C} \cdot \mathrm{I} 8$.

D. $\frac{1}{3} \frac{3}{9} \cdot P \cdot I 6 \cdot V \cdot 6 \cdot A \cdot \frac{3}{23} \cdot C \cdot 20$,

faxatilis, IS. C. Spinis pinnae dorfalis I4, pinnis ventralibus acuminatis, cauda bifida, dentibus emarginatis.

Muf. Ad. Fr. I. p. 64. Chaetodon cauda bifurca, flfciis 5 albis.

Anoen.acad.I. p. 3I2. Spa-

D. $\frac{1}{2} \frac{4}{6} \cdot P \cdot 15 \cdot V \cdot \frac{\pi}{6} \cdot A \cdot \frac{2}{15} \cdot C \cdot I 5 \cdot$ rus fafciis 5 transuerfis fufcis.

D. $\frac{12}{2} \cdot$ P.IS. V. I. A. $\frac{2}{14} \cdot$ C. I5. Gron. me. I. n. 39. Idem. D. $\frac{13}{2} \frac{25}{}$ P.I7. V. $\frac{1}{6} \cdot A \cdot \frac{2}{14} \cdot$ C.I8, Habitat in India.

Habitu toto Percae a congeneribus recedit. 


rotundus. I9. C. fpinis pinnae dorfalis 23 , facciis quinque pallidis.

Muf:Ad. Fr. r.p. 64. Chaetodon rotundatus cinereus, falciis 5 .

Habitat in indiis. D. $\frac{2}{2} \frac{2}{6} \cdot$ P. IO. V. $\frac{1}{6} \cdot A \cdot \frac{2}{14} \cdot C \cdot \therefore$

lanceol-20. C. falciis tribus: oculatus. ri pecturali longitudinalique, cauda integra.

Edro au. 210. t. 210. Gua. pena.

Irabitat in India.

Corpus oblongum, lanceolatum: fafciae nigrae margine grifere 3; prima transuerfa per oculos; fecunda transuerga per thoracem; tertia a pina dorjali ane tiriori ad piman caudae.

IfI. SPARVS. Citput: Dentes incifores vel laniarii robufi; molares obtufufculi, conferti. Mombr. lranch. radiis V. Oper. ctilit fquanofa.

Corpus comprefium.

* Macula nigra notati.

Aurata. I, S. lumula aurea intcr oculos. D. $\frac{3 \pi}{2} \cdot$. P. I5. V.6. A. $\frac{3}{15} \cdot$ C.I7. Art. gen. 25. Jym.63. Sparus

dorto acutifimo, linea

arcuata aurea inter oculos. D. 24. P.17. V. 6. A. I4. C. ..

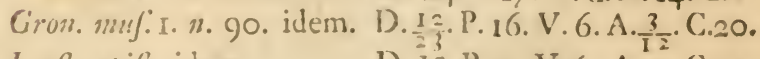
Locf. epift. idem.

Hafjelqu. itin. 337. idem. D. $\frac{21}{2} \cdot$ P. I6. V.6.A. $\frac{2}{14} \cdot$ C.22. llabitat in Mari Mediterraneo et Oceano imbr Euro. pam Ameriçumque.

In meo eximplavi masula nigra ad caudam.

Romanis olinn fretiofiffnuts Sirgi audoritasi; Vener: fircer. 
annula- 2. S. oce'lo nigro fubcaudali, ris. corpore flauefcente. Art. ggen. 37. Jyn. 57. Sparus vnicolor tauefcens, maciala nigua annulari ad caudam.

Habitat in M. Adriatico, infero.

Sargus. 3. S. ocello fubcaudali, corpore fafciis nigris.

Art. gen. 37. Jjn. 58. SpaD. $\frac{12}{2} \cdot$ P. I6. V.6.A. $\frac{3}{16} \cdot$ C.I\% rus lineis transueriis varius, macula nigra intigni ad caudam.

Habitat in M. infero.

Melanu- 4. S. ocello nigro caudae, corrus. pore lineis longitudinalibus.

Art. gen. 37. Syn. 58. Sparus lineis longitudinuisbus varius, macula vtrinque ad caudam.

Habitat in M. infero.

Smaris. 5. S. ocello nigro laterali, pinnis pectoralibus caudaeque rubris.

Art. gen. 36. Jyn. 62. Sparus macula nigra in vtroque latere medio, pinnis peEtoralibus caudaque rubris. Habitat in Europa auftrali.

Maena. 6. S. ocello fufco laterali, corpore variegaro. Art. gen. 36. Jyn. 61. Sparus varius, macula nigricante in medio latere, dentibus 4 maioribus. Habitat in M. mediterraneo.

faxatilis. 7: S. ocello fubcaudali, corpore candicante. 


Muf.Ad.Fr. r. p. 65. Sciae-

na ocello ad bafin pinnae

caudse.

Giron. m::1: 2. n. I85. t. 6.

D. $\frac{19}{33} \cdot P \cdot 16 \cdot V \cdot \frac{1}{6} \cdot A \cdot \frac{3}{12} \cdot C \cdot 16$.

$f: 3$. Sparus roftro plagioplateo, rufefcens, macula nigra iride alba ad caud'am fubrotundam. D. $\frac{17}{3} \frac{1}{I}$ P.I7. V. $\frac{1}{6} \cdot A \cdot \frac{3}{11} \cdot C \cdot I 7 \cdot$

Havitat Surinami.

Orphus. 8. S. ocello fubcaudali, capite rufelcente, cauda integra. $t$

Art. gen. 37. Jyn. 63. Sparus varius macula nigra ad caudam in extremo aequalem. D. ז0. Hobitat . .

* * Rubri.

Hurta. 9. S. cauda bifida, corpore fafciis transuerfis rubris, dentibus laniariis exfertis. D. $\frac{1 \pi}{2} \cdot$ P. I6. V. 6.A. $\frac{3}{9}$. C. 17 . Habitat in M. Mediterraneo. An Dentex 17 ?

Erythri- 10. S. cauda fubintegra, cornus. pore rubro. Muf: Ad. Fr.
2. p...
Loefl. cpifl. $m \int c$.
Art. gen. 36. Jyn.59. Spa-
D. $\frac{12}{2} \cdot$ P.I6.V.6.A. $\frac{2}{13} \cdot$ C. I8.
rus totus rubens, iride argentea.
Habitat in M. Meditertaneo, Ameticano.

Pagrus. Ir. S. rubefcens, cute ad radicem pinnarum dorfi ct ani in finum producta. Art. gen. 36. Jyn.64.

Ilabitat in Europa aufbrali, 


\section{* * * Linleati.}

Boops. I2. S. lineis longitudinalibus obfcuris: inferioribus quatuor aureis argenteisque. Ait. gen. 36. Jyn.6r. Sparus lineis verinque 4 aureis ac argenteis longitudunibus parallelis. Habitat in M. intero.

Cantha- I3. S. cauda immaculata, corxนร. pore lineis longicudinalibus luteis. Art. gen. 36. Jyn. 58. Sparus lineis vtrinque luteis Iongitudinalibus paralle lis, iride argentea. Habirat in M. infero.

Chromis. If. S. cauda bifida, radio ventralium fecundo fetaceo. Art. gen. 37. fyn. 62. Sparus officulo fecundo pin-。 narum ventralium in longam fetam qualiproduko. D.23. P.I7.V.6. A. I2. C. Habitat in Europa auftrali.

Salpa. I5. S. cauda bifida, lineis fuluis longitudinalibus vtrinque vndecim.

Art.gen. 38. Jyn.60. Sparus D. $\frac{1}{2} \frac{x}{8} \cdot$ P.I6. V.6.A. $\frac{3}{17} \cdot C \cdot I 7$. lineis vtrinque vndecim aureis parallelis longitudinalibus.

Iubitat in M. Mẹditerraneo.

Syrnagris. I6. S. cauda bifida rubra, corpore purpurafcente lineis vtrinque 7 aureis.

Catesb. car. 2. p. I7.t. I7. Salpa purpurafcens variegata.

Habitat in America feptentrionali. 


**** Varii.

Dentex. I7. S. cauda bifida, corpore variegato, dentibus quatuor maioribus.

Art. geir. 36. jyn. 6o. Sparus varius, dorfo acuto, derribus 4 maioribus. D.90. P. -. V. .- A. I0.C... Habitat in $M$. infero et fupero.

Spinus. I8. S. cauda bifida, fpina dorfali recumbente. D. $\frac{13}{2} \cdot$ P.I6.V. $\frac{1 \cdot 1}{5} \cdot A \cdot \frac{7}{\frac{1}{6}} \cdot$ C. 16.

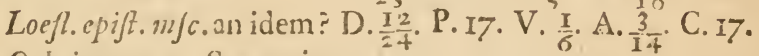
Osb.iter.273. Sparus is-
uanenlis.
D. $\frac{13}{2} \cdot$ P. I5. V. $\frac{2}{5} \cdot A \cdot \frac{7}{15} \cdot$ C. IS.

Habitat in Indiis.

Corpus lituris caeruleis repandis variis piatum.

virgini- I9. S. cauda bifida, fafciis

cus.

duabus nigris transuerfis, lineisyuce caeruleis plurimis.

D. $\frac{11}{2} \cdot P \cdot I S \cdot V \cdot T \cdot A \cdot \frac{3}{13} \cdot$ C.IS.

Habitat in America Septentrionali. Mut: De Geer.

Opercula fibjerrata. Fafcia prior ab bumeris per oculos ad maxillam inferiorem, altera ab bameris per. latera thoracis ad pinnas pecturales. Lirecae cairuleae plures, longitudinales, parallelae. Caudae pinna bilub.z, obtuja.

Mormy- 20. S. cauda bifida, fafeiis arrus. genteis nigrrisugue plurimis. Art.gen. 37.Jyn.62. Sparus maxilla luperiore longiore, lineis vtrinque duabus nigris transuertis purallelis. D.23. P. I4. V.6.A. I3. C. -Hafjelizu. itin. 335. Sparus
Minrmyrus.
Habitat in M. infero.
D. $\frac{1}{24} \cdot P \cdot 15 \cdot V \cdot y_{0} \cdot A \cdot \frac{3}{13} \cdot C \cdot I S$.

eapiftra- 2I. S. cruda inregra, corpo-

tus. re albo-rericulato. D. $\frac{n}{2} \cdot$ P. I2.
Hathitat in Ancicica. Muf: De Cirs.

D. $\frac{n}{2} \cdot P \cdot 12 \cdot V \cdot I, A \cdot \frac{1}{16} \cdot C \cdot 14^{\circ}$

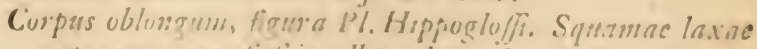

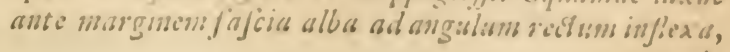


vnde pifcis reticulatus. Dentes primores magni 2 juperiores, 4 inferiores. Pina dorjalis longitudine fere dorfi.

galilaeus. 22. S. cauda integra, corpore fupra virefcente, fubtus albo. $t$

Huffelqu. itin. 343. $n \cdot 7^{6}$

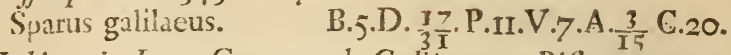

Habitat in Lacu Genezareth Galilaeae. Pifcatura prodigioja Lucae $V$. ex boc pifce.

142. LABRVS. Caput: Dentes acuti.

Menzbr. branch. radiis VI, Opercula fquamofa.

Corpus: Pinnae dorfalis radii poftce ramento filiformi aucti.

* Cauda bifurca.

Scarus. I. L. appendicibus transuerfis ad caudae latera. $\dagger$

Arí. Jyn.54. Scarus auktorum.

Habitat in Graeciae littoribus, Siciliam tenus.

Sub Tiberio Clandio in deliciis, raminans; iecinora in Vitellii patina f: clypeo mineruae. Pifcis bodie ob fcurus.

cretenfis. 2. L. dentibus 4 virefcens, cauda bifurca. Art. gen.

34. $\int y n \cdot 57 \cdot+$

Habitat in Creta et adiacentibus.

Anthias. 3. L. totus rubefcens, cauda bifurcia. Art. Jyn. 54. D. $\frac{1}{1} \frac{0}{9}$.

Catesb. car. 2. p. 25.t. 25 . Anthea IV. Rondel.

Habitat in Europa meridionali et America.

Hepatus, 4. L. maxilla inferiore longiore, canda bifurca, lineis virinque transuerfis

sigris.

Labri et Spari genera ob affinitatem etiamnum difficilius diftingunntur. 


nigris. Art. gen. 35. D. 10. P. 13. V.6. A. 9. C. -
Jyin. 53.

Habitat in Mari Mediterranto.

Pinna dorlahs poft radios fipinofos, parte fuperiore, notatur macula nigra. Rai.

grrifeus., 5. L. cauda fubbifida, corpore 1ubgrifeo. $\dagger$

Catesb. car. 2. p. 9. t. 9. Turdus pinnis branchialibus carens, *

Habitat in America.

Catesbaeus absque pinnis pectoralibus pinxit ; an rite?

lunaris. 6. L. pinna caudae medio truncata, doráli aniq̧ue linea purpurea, lablis plicatis. $\quad$ D. $\frac{8}{23}$. P.15.V. $\frac{0}{6} \cdot$ A. $\frac{2}{15} \cdot$ C. 16.

Gron. muf. 2. n. ISO. t. 6 . f. 2. Labrus oblongus, cauda bifurca, capire pur-

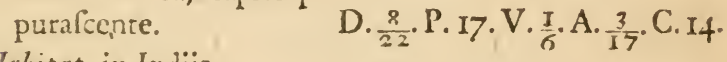

Habitat in Indiis.

opercu- 7. L. cauda bifida, corpore laris. fafciis 10 , maculaque 0 perculorum fufcis. Cbin. Lagerftr.24. $\quad$ D. $\frac{13}{20} \cdot$ P.16.V. T. A. $\frac{15}{2} \cdot$ C. 16.

Habitat in Alia.

Pauo. S. L. viridi caeruleo fanguineo canoque varius, cauda bifida.

Art. gen. 34. Syn. 55. Labrus pulchre varius, pinnis petoralibus rotundatis.

Haljeligu. iter. 344. n. 77 .

Labrus Pauo. B.6. D. 31.P.14.V.
Habitat in M. Mediterraneo ad Syrian.

auritus. 9. L. cauda bifida, operculis branchiarum pinniformilus.

Habitat in Philadelphia. Muj: De Geer.

D. $10 \cdot$ P.15.V.6.A.13. C. 17 . 10. L, 
falcatus. IO. L. pinna dorfali analique radiis quinque primis inermibus falcata. D. $\frac{7}{27} \cdot \mathrm{P} \cdot 17 \cdot \mathrm{V} \cdot 5 \cdot \mathrm{A} \cdot \frac{3}{20} \cdot \mathrm{C} \cdot 20$. Habitat in America. Muf. De Geer.

Corpus latitudine Bramae, argenteum. Radii 5 primi e mollibus $R$. dorfalis analisque elongati, fequentibus aequalibus, vnde bae pinnae falcatae. Dentes acuti Junt. Pinnae ventrales pariae.

rufus. Ir. L. cauda lunata, corpore toto fuluo. Locfl. D.23.P.I7.V.6.A.I2.C.I6.

Catesb.car. 2. p. II.t. Ir. Turdus flauus.

Habitat in America.

* Cauda integra ( Secundum Sinas dorfales).

matgina- 12. L. fubfufcus, margine pin-

lis. narum dorfi pectoraliumque fuluo. Lojl.epift. D. $\frac{2}{22} \cdot$ P.17.V.6.A. $\frac{3}{12} \cdot$ C.I7. Habitat in Pelago.

ferrugi- 13. L. ferrugineus immacuneus.

zulis

Iulis.
latus, cauda integra.
D. $\frac{2}{28} \cdot P \cdot I 6 \cdot V \cdot \frac{1}{6} \cdot A \cdot \frac{3}{17} \cdot C \cdot I 7 \cdot$ Habitat in India.

I4. L. lateribus caerulefcentibus: vitta longitudinali fulua vtrinque dentata. D. $\frac{0}{2} \cdot$ P.I3. V. $\frac{x}{6} \cdot$ A. I3.C.I2. Art. gen. 34. Jju. 53. Labrus palmaris varius, dentibus 2 maioribus maxillae fuperioris.

Gron. muyf:2. 2 . I84. L. obD.2I. P.I4. V. G.A.I4.C. $=$ longus nigricans, lateribus Jinea aiba vtrinque finuata varius, cauda indiuifa. D. $\frac{9}{21} \cdot P \cdot I 3 \cdot V \cdot \frac{1}{6} \cdot A \cdot \frac{3}{15} \cdot C \cdot I G$. Habitat Genuae.

Formolifinums pifcis Europaeorum ob colores varios.

paroticus.

15. L. cauda integra, linea laterali curua, pinnis rufis, operculis coccineis. Muf.
Ad. Fo . 2. p...
D. $\frac{9}{21} \cdot$ P.I2. V.6.A.I4.C.I4.

Hubitat in India. 


fuillus. 'T6. L. pinna dorfi ramentacea, macula nigra fupra caudam, fpinis dorfalibus 9 .

It. Wgot. 179. Sparus Berginyltra.

Habitat in O. Europaeo.

ftriatus. 17. L. pinna dorfi ramentacea lineis albis fufcisque.

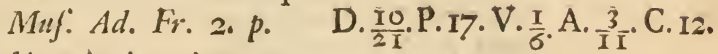
Habitat in America.

Guaza. 18. L. fufcus, cauda rotundata, radiis membranam fuperantibus. Loefl. epif. D. $\frac{11}{27}$. P.16.V.6.A.13.C. 15. Habitat in Pelago.

ocellaris. 19. L. pinna dorfi ramentacea, ocello in medio ad balin caudae. $M u v$. Ad. Fr. 2. p... D. $\frac{14}{24}$ P. $15 \cdot V \cdot \frac{1}{6} \cdot A \cdot \frac{T}{13} \cdot C \cdot 13 \cdot$ Habitat .

Tinca. 20. L. roftro furfum reflexo, cauda in extremo circulari. Art. gen. 33. Jyn. 56. D. $\frac{15}{26} \cdot$ P. I4.V.6.A. $\frac{2}{13} \cdot C . \cdots$

Habitat in M. Britannico.

bimacu- 2r. L. pinna dorfi ramentalatus. cea, macula fufca in latere medio et ad caudam. Muf. Ad. Ir. r. p. 66. Sciaena macula fufca in medio corporis et fupra balin caudae.

Habitat in M. Mediterraneo. D. $\frac{15}{6} \cdot P \cdot 15 \cdot V \cdot \frac{1}{6} \cdot A \cdot \frac{4}{12} \cdot C \cdot \cdots$ puncta- 22. L. pinna dorfi ramentatus. $\quad$ cea, lineis parallelis fulcopunstatis. 
Muf. Ad. Fr. I. p. 66. Sciae$n$ a lineis longitydinalibus plurimis fufco punctaris. D. $\frac{15}{24} \cdot P \cdot 14 \cdot V \cdot \frac{\pi}{6} \cdot A \cdot \frac{3}{19} \cdot C \cdot 16$.

Gron. mif. r. 21. S7. Labrus brunneus, officulo fecundo p. ventralium fetiformi.

Habitat Surinami.

D. $\frac{15}{25} \cdot P \cdot I 5 \cdot V \cdot \frac{x}{6} \cdot A \cdot \frac{4}{12} \cdot C \cdot I 8$.

Melops. 23. L. pinna dorfi ramentacea anique varicgata, lunula fufca pone oculos. Muf: $A d . F r \cdot 2 \cdot p \cdot \ldots \quad$ D. $\frac{1}{2} \frac{6}{3} \cdot \mathrm{P} \cdot 13 \cdot \mathrm{V} \cdot \frac{\mathrm{T}}{6} \cdot \mathrm{A} \cdot \frac{3}{13} \cdot \mathrm{C} \cdot \mathrm{I} 2$

Habitat in Europa anfirali.

niloticus. 24 . L. cauda integra, pinnis dorfi ani caudaeque nebulatis. Muf. Ad. Fr.

2. p... D. $\frac{17}{30} \cdot$ P.I4.V. $\frac{1}{6} \cdot A \cdot \frac{3}{12} \cdot C \cdot 17$.

Huffelqu. iter. $346 . n \cdot 78$. Labrus niloticus.

Habitat in Aerypto.

D. $\frac{17}{30} \cdot P \cdot 15 \cdot V \cdot \frac{1}{6} \cdot A \cdot \frac{2}{12} \cdot C \cdot 20$.

offfagus. 25. L. labiis plicatis, pinna dorfali radiis 30.

Habitat in Europa.

D. $\frac{\pi}{3} \frac{7}{1} \cdot P \cdot I 5 \cdot V \cdot \frac{T}{6} \cdot A \cdot \frac{3}{15} \cdot C \cdot 13 \cdot$

rupeftris. 26. L. pinna dorfi ramentacea antice macula fufca. Mul:Ad. Fr. r. p. 65. Sciacna margine fuperiore caudae macula fufca notato. D. $\frac{x}{2} \frac{7}{6}$ P. I $14 \cdot V \cdot \frac{\pi}{6} \cdot A \cdot \frac{3}{10} \cdot C \cdot 13 \cdot$

Habitat . .

Onitis. 27. L. pinna dorfi ramentam cea, abdomine cinereo fufcoque maculato. Minf. Ad. Fr. 2. p. ...

Habitat. .

D. $\frac{17}{27} \cdot P \cdot 15 \cdot V \cdot \frac{T}{6} \cdot A \cdot \frac{3}{1} \tilde{I}^{\cdot}$ C.I 4 .

viridis: 28 . L. viridis: linea vtrinque

caerulea. Art. gen. 34. D. $\frac{18}{30^{\circ}}$

Habitat in M. Mediterraneo. 


lufcus. 29. L. cauda integra pinnis omnibus flauis, palpebra fuperiore nigra. Muj: Ad. Fr. 2.p...

Habitat . .

D. $\frac{18}{3} \frac{8}{1} \cdot$ P.14.V.6.A. $\frac{3}{14} \cdot$ C.14

livens, 3०. L. pinna dorfi ramentà cea, corpore fufco-liuido. Muf: Ad. Fr. 2.p. D. $\frac{18}{3} \cdot$ P.14.V. $\frac{1}{6} \cdot$ A. $\frac{3}{1 \frac{1}{2}} \cdot$ C. II. Habitat . .

Turdus. 3r. L. oblongus viridis, iride aurea. Art. gen. 34 . $\int y n \cdot 57$.

Habitat in M. Europaeo.

\section{D. $1 \frac{9_{3}}{3}$}

exoletus. 32. L. pinna dorfi ramentacea, corpote lineis caeruleis, pinna ani pinis 5. D. $\frac{19}{2} \cdot P \cdot 13 \cdot V \cdot \frac{1}{6} \cdot A \cdot \frac{5}{13} \cdot C \cdot I_{3}$ Habitat in Oceano Atlantico.

chinenfis. 33. L. pinna dorfi ramentaced, corpore liuido, vertice retulo.

Habitat in Alia. D. $\frac{19}{2} \cdot P \cdot I 3 \cdot V \cdot \frac{1}{6} \cdot A \cdot \frac{5}{12} \cdot C \cdot 12$.

linearis. 34. L. oblongus, pinnae dorfulis radio vitimo inermi. Amver. acad. I. p.315. D. $\frac{20}{21} \cdot$ P.12. V. ․․‥ A. 5. C.I2. Habitat in Indiis.

* * Cauda integra ( * pinis dorfalibus adbuc ignotis).

mixtus, 35. L. e flauo caeruleoque varius, dentibus anterioribus maimibus. Art. gen. 34. $\int j n .57$.

Habitat Liburni.

fuluus. 36 . L. cauda intezra, corpo. re fuluo.

Catesb. car. 2. p. 10. f. 2. Turdus cauda conuexa. Habitat in America. 
radiatus. 37 , L. cauda integra, oculis radiatis, corpore caeruleo maculato, fifcia pectorali caudaeque fulua,

Catesb. car. 2. p. 12. t. 12. f. I. Turdus oculo radiato.

Habitat in America.

varius. 38 . L. e purpureo viridi caeruleo nigroque varius. Art. gen. 35. Jyn. 55 .

Habitat in M. Mediterraneo.

Merula. 39. L. caeruleo - nigricans. Art. Iyn. 55 .

Habitat in Europa.

Cynae- 40. L. luteus, dorfó purpu. dus. veo, pinna a capite ad caudam continua. Art. fyn. 56.

Habitat in M. Mediterranco.

143+ SCIAENA. Caput . .

Membr. branch. radiis VI. Opercula fquamofa er torum caput. Corpus: Foffula dorfi pro pinna dorfali recondenda.

Cappa. I. S. capitis lateribus fquama-

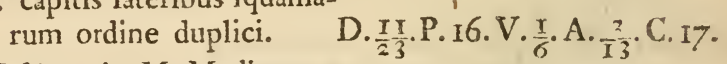
Habitat in M. Mediterraneo.

Lepifma. 2. S. pinna dorfali intra duo folia fquamea recondenda.

D. $\frac{10}{1} \frac{1}{9} \cdot P \cdot I I \cdot V \cdot \frac{1}{6} \cdot A \cdot \frac{3}{11} \cdot C \cdot 13 \cdot$

Habitat...

Sciacnae genus medium eft inter Labros et Percas, vix fufficicnter diffinctum. 


vnima- 3. S. macula fufca in medio culata. vtriusque lateris. $\quad$ D. $\frac{11}{21} \cdot P \cdot 15 \cdot V \cdot \frac{\pi}{6} \cdot A \cdot \frac{3}{12} \cdot C \cdot 17$. Habitat . .

Vmbra, 4. S. nigro varia, pinnis ventrahibus integerrimis. Art. gen. 39. Jyn. 65. Hafjelqu. iter. $352 . n .80$. Sciaena vmbra. Habitat in M. Mediterraneo.

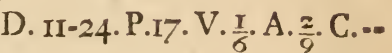
D.10-26.P.18.V. $\frac{1}{6} \cdot A \cdot \frac{z}{9} \cdot C .18$. cirrofa, 5. S. maxilla fuperiore longiore, inferiore cirro vnico. Art.gen. 38 .

Gron. mul. 1. 11. 92?

Habitat in M. Mediterraneo $c t$ infero.

144. PER CA. Caput: Opercula fquamofa ferrata. Membr. branch. radiis VII. Corpus pinnis fpinofis.

* Pinnis dorfalibus 2 diftinctis.

fluuiati- I. P. pinnis dorfalibus diftinlis. ctis: fecunda radiis 16. Fn. Jilec. 285 . Avt. gen. 39. Jynt. 66. Jpec. 74. Perca lineis vtrinque fex nigris, pinnis ventralibus rubris. Gron. muf: I. n. 96. idem D.14,16. P.14.V. $\frac{1}{6} \cdot A \cdot \frac{2}{12} \cdot C \cdot 17$. nomen.

D.I4,I4. P. I3 V. 6. A. II. C. 17 . Habitat in Europae Lacubus imprimis.

Onarium vrnicum. Pijcatur in determinato profindo; cibo falusaris cum Petrojelino laudatur.

Lucio- 2. P. pinnis dorfalibus diftinperca. Ctis: fecunda radiis 23. Fn. Jitec. 285. 


\section{PISCES 'THORACICI. Perca,}

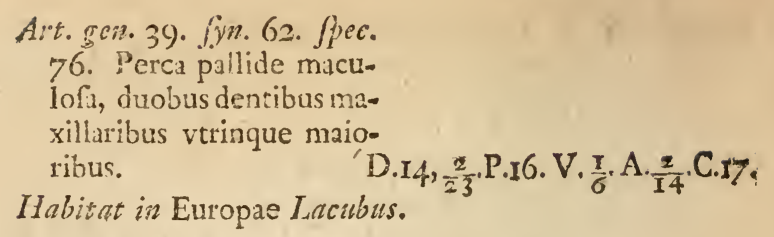

Afper, 3. P. pinnis dorfalibus diftin-

Qis, fecundae radiis $\mathrm{I}_{3}$. Art.gen. 40. (yn.67. Perca lineis vtrinque 8. f. 9 . transuerfis nigris. Ilabitat in Europa ainfraliore.

D. 8, I3.P.14. V.5. A.I2.C.:-

Labrax. 4. '. pinnis dorfalibus diftinAis, fecundae radiis I4. Gron. Act. Vp $\int$. $1750 . p$. 39. t. 4 . Art. gen. 4I. fyn. 69. PerD.9, $\frac{1}{14} \cdot$ P.I9.V. $\frac{1}{6} \cdot A \cdot \frac{3}{14} \cdot$ C.I8. ca radiis pinnae dorfalis fecundae $I_{3}$, ani I4. D.9,I3.P.I5. V.6.A. $\frac{3}{14} \cdot$ C. .Habitat in Europa auftraliore.

Hic, Muraena Helena, Myxo in Tripatino; Romae optimus inter duos pontes.

nilotica. 5. P. pinnis dorfalibus fubdiftiotis, caudae integra. Mif. Ad. Fr. 2. p. D. $8, \frac{x}{9} \cdot$ P.14. V.6.A. $\frac{3}{13} \cdot$ C. 15. Haffolqu. iter: 359. n. 83 . Perca nilotica.

Habitat in Nilo. Hajelnuift. D.8, $\frac{1}{13} \cdot P \cdot I 6 \cdot V \cdot \frac{I}{6} \cdot A \cdot \frac{3}{12} \cdot C \cdot 20$. * Lorjo monopterygio, cauda indinifa.

marina. 6. P. pinnis dorfalibus vnitis; radiis $\mathrm{XV}$ fpinofis, XIV muticis, corpore lituris variegraco.

Art. gcn. 40. fyn. 68. Per-

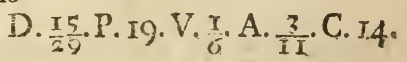
Cal ineis vtrinque 7 trans-

* ueris nigris, du\&ibus nitniaccis caerulcisque in $\mathrm{ca}$ pite et antica ventris. Inbitat in Norvegia, Iralia. D. $\frac{10}{2} \frac{1}{5}$ P... V. .. A. $\frac{3}{10} \cdot C . .$. 7. P. 




\section{PISCES THORACICI. Perca:}

nobilis. 7. P. pinnis dorfalibus vnitis,

cauda integra, corpore argenteo fafciis octo fufcis.

D. $\frac{1}{25} \cdot P \cdot 15 \cdot V \cdot \frac{1}{6} \cdot A \cdot \frac{3}{10} \cdot C \cdot 17 \cdot$ Habitat in America Septentrionali. Mus: De Geer. Radii jpinofi dorealis laterbus argentei junt.

polym- 8. P. pinnis dorfalibus vnitis,

ก.. corpore nigro: fafciis 3

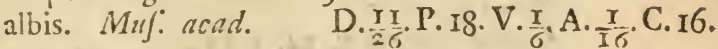
Habitat in Indiis.

Coniungit bace Percas, Labros, Sparos, Cibatodontes, fed opercula jpinoja ad Percann referunt.

cottoides. 9. P. pinnis dorfalibus vnitis, cauda indiuifa, pinnis omnibus lineis duabus punEtatis.

D. $\frac{\pi 4}{20} \cdot P \cdot I 4 \cdot V \cdot \frac{x}{5} \cdot A \cdot \frac{3}{10} \cdot C \cdot I 2$.

Habitat . .

philadel- IO. P. pinnis dorfalibus con-

phica. natis: radiis XI fpinolis,

IX muticis.

Habitat in America.

D. $\frac{\mathrm{II}}{20} \cdot \mathrm{P} \cdot 16 \cdot \mathrm{V} \cdot 6 \cdot$ A. $\frac{3}{8} \cdot \mathrm{C} \cdot \mathrm{II}$.

mediter- II. P. pinnis dorfalibus vni-

ranea. tis, reliquis fuluis, ma-

cula nigra ad pectorales.

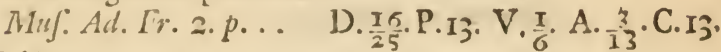

Habitat . .

vittata. 12. $P$. pinnis dorfalibus vni-

tis, fafciis 5 longitudi-

nalibus albis fufcisyue.

Mul: Ad. Fr.2.p. D. $\frac{1}{18}=$ P. IS. V. $\frac{T}{6} \cdot A \cdot \frac{3}{1=} \cdot$ C. $17 \cdot$

Habitat . .

punctata. 13. P. pinnis dorfalibus vni-

tis, cauda integri, cor-

pore cieruleo punctato.

Catesb.car.2. p.7.t.7.7.1.

Perca marina punctata.

Ilabitat in America. 
guttata, I4 , P. pinnis dorfalibus vnitis, cauda integra, corpore punctis ianguineis adsperio.

Marcgr. braf. r6g. Cugupugvacu.

Sloan. iant. 2. p. 280 . $t$. $247 . f$. 2.

IVill. icht. 303. t. I.

Rai. pifc. 127 .

Catesb. car. 2. p. I4. t. I4.

Habitat in America.

Scriba, $\quad$ 15. $P$. pinnis dorfalibus vnitis, cauda integra, pinnis caudae pectoralibusque flauis, capite fripto. Muf. Ad. Fr. 2. p. . .

Habitat . .

$$
\text { D. } \frac{10}{2} \frac{0}{5} \cdot P \cdot 13 \cdot V \cdot \frac{1}{6}, A \cdot \frac{3}{10} \cdot C \cdot 15 \text {. }
$$

* * Dorfo monopterygio, cauda bifida.

veneno- I6: $P$. pinnis dorfalibus vnitis,

fa. pectoralibus apice flauis, cauda lunata, corpore punctis fanguineis.

Catesb, car. 2. p. 5. t. 5 . Perca marina, venenofa punctata.

Habitat in America.

melanu- I7. P. pinnis dorfalibus vni$\mathrm{Fa}_{\mathrm{s}}$ tis, cauda bifida nigra margine albo, corpore lineis luteis.

Catesb. car. 2. p. 7. t.7. $f$. 2. Perca marina cauda nigra,

Habitat in America.

gibbofa, 18. P. pinnis dorfatibus vnitis, cauda bifida, abdomine luteo, operculis Ariaris apice nigro ful uoque. 




\section{PISCES THORACICI. Perca.}

Catesb. car. 2. p. 8. t. 8 . $f \cdot 3$. Perca fluuiatilis gibbofa, ventre luteo.

Habitat in America.

faltatrix. 19. P. pinnis dorfalibus vnitis, cauda bifurca poftice rubra, abdomine luteo grifé-lineato.

Catesb.car.2.f.8. t.8.f.2. Perca marina faltatrix.

Habitat in America.

Atigma, 20. P. pinna dorfali ramentacea, operculis inuftis. Muj: acad.

Habitat in India.

D. $\frac{18}{27} \cdot P \cdot 13 \cdot V \cdot \frac{1}{6} \cdot A \cdot \frac{9}{19} \cdot C \cdot 17$.

diagram-2I. P. pinnis dorfalibus vni-

ma. tis, corpore lineis luteis.

Gron.muf: I. n.88. Sparus lineis longitudinalibus luteis varius, aculeis $\mathrm{XI}$. in pinna dorí.

D. $\frac{11}{2} \cdot P \cdot 13 \cdot V \cdot \frac{1}{6} \cdot A \cdot \frac{3}{11} \cdot C \cdot 18$.

Habitat . .

ftriata. 22. P. pinnis dorfalibus vnitis, cauda bifida, corpore ftriato. D. $\frac{1}{2} \frac{3}{8} \cdot P \cdot 15 \cdot V \cdot \frac{1}{6} \cdot A \cdot \frac{3}{11} \cdot C \cdot 17 \cdot$ Habitat in America feptentrionali. Mus:: De Geer. Opercula fubjerrata. Radius fecundus analis validiffumus. Caida nigra non eft, qua differt a P. Melanura.

lineata. 23. P. pinna dorfali ramentacea, corpore lineis 5 albis finfcisque.

Milj: Ad. Fr. I. p. 66. Sciaena fifciis 5 longitudinalibus albis fufcisque a!rernis. D. $\frac{17}{33} \cdot P \cdot 15 \cdot V \cdot \frac{1}{6} \cdot A \cdot \frac{2}{10} \cdot C \cdot 16$

Habitut . .

rhomboi-24. P. lineis, 7 luteis, pinnis dalis. flauis, cauda bifurca.

Catisb. 
Cateśr.car.2.p.4.t.4. Perca marina rhombordalis fafciata.

Erown. iann. I46. Sparus Atris longitudinalibus varius, dentibus anteriori-

Fus latioribus compreifis aequalibus acutis.

Habitat in America.

Cernua. 25. P. pinnis dorfalibus vnitis radiis 27 , fpinis 15 r cauda bifida. Fn. Jieec. 286. Avt. gen. 40. Jyn. 68. Jpec. 77. Perca dorfo monopterygio, capite cauernofo.D. I $\frac{15}{2} \cdot$ P. I5. V. $\frac{1}{5} \cdot A \cdot \frac{2}{8} \cdot C .$. Gron.mef. r. 12. 94. idem. D. $\frac{15}{2}$. P. -- V.6. A. $\frac{2}{7} \cdot$ C.16. Habitat in Europae Lacubus.

Schraet- 26. P. pinnis dorfalibus vnitis,

fer. lineis corporis nigris. Ait. gein. 40. Jyn.68. Perca dorfo monopterygio, lineis verinque longitudinalibus nigris. D. $\frac{18}{30} \cdot$ P.IG.V.G.A. $\frac{2}{8} \cdot$ C. . Habitat in Europa auftrali.

argentea. 27. P. pinnis dorfalibus vnitis, cauda bilida, naribus tubulolis. Mul. Ad. Fr.2.p...D. $\frac{12}{22}$. P. I2.V. $\frac{1}{6} \cdot$ A. $\frac{3}{11} \cdot$ C.I7. Habitat . .

Cabrilla. 28. P. pinnis dorfalibus vnitis, cauda bifida, fafciis Jongitudinalibus 4 fanguineis. Muf: Ad. Fr. 2.p... D. $\frac{10}{2} \frac{1}{4}$ P.16.V. $\frac{1}{6} \cdot$ A. $\frac{3}{10} \cdot$ C.17. B. Muf.Ad. Fr. 2. p. . . . Ferca lituris flauis violaceisque variegata. Habitat . . D. $\frac{10}{24}$ P. I4. V.6.A. $\frac{3}{10} \cdot$ C.16.

Radula. 29. P. pinnis dorfalibus vnitis, cauda bifida, corpote punctis albis lineato. 


Amocn. acad. I. p. 315. La-

brus immaculatus, pin-

nae dorfalis radiis to fpinofis.

Habitat in India.

D. $\frac{11}{20} \cdot$ P.I2. V, 6.A. $\frac{3}{13} \cdot$ C.I7,

Squanace cremulatae.

145. GASTEROSTEVS. Caput . .

Menubr. branch.radiis III. Arted.

Corpus ad caudam vtrinque carinatum. Aculei diftinéti ante pinnam dorfi.

aculeatus. I. G. fpinis dorfalibus tribus. Avt.gen. ${ }^{2} \int \mathrm{j} n \mathrm{n} . \mathrm{so} \cdot \int \mathrm{pcc}$. 26. Fn. firec. 276 . Habitat in Europa.

Dustor. 2. G. fpinis dorfalibus quatuor. B.7.D.4,27.P.IS.V.6.A.16.C.19. Halfelqu, iter. 366. Scomber Ductor.

Osbeck.itir.73. Scombe:

D.3.I2.P.IO.V. $\frac{x}{2} \cdot A \cdot \frac{1}{9} \cdot C \cdot 12$, DuRtor.

- Ait. Stockh. $1755 \cdot p \cdot 7 \mathbf{x}$.

D. $\frac{4}{2} \cdot$ P. 20.V.5.A.16.C.16. idem.

Habitat in Pelago, Satelles Squali.

Midius quafi intir. Ciafleroficos et Scombros; at corsuenit cum illis finis ante piman dorgalem diftinetis it cum C. Pungitio crudae lateribus carinatis, quamuis differat a Ciaftereftcis Menbrans bianclioglega.

occider- 3. G. fpinis dorfu!ibus feptem,

talis. fpinisque duabus ante pinn.m analem. D.7,11.P.7.V.G.A.2, $\frac{1}{7}$.C.IG. Habitat in America. Muf. Do Cour. 
ouatus. 4. G. fpinis dorfalibus feptem: prima recumbente, corpo$\begin{array}{lll}\text { re ouato. I } & \text { B.6.D.7,20.P.I6.V.6.A. } 2, \frac{1}{17} \cdot C_{2} 2 .\end{array}$ Habitat in Afia.

Pifciculus ouato oblongus, compreffus, figura Chatodontis. Maxillue et labiorum dentes fiabri. Spinae in dorfo 7 ante pinnam dorfalem, fid difinctae et alternatim ad latera directae; barum prima minima, 2da paulo longior. Spinae 2 validae unte pinnam ani. Differt a Gafterofteis reliquis membr. branch. radiis 6 , nec 3 ; caret foutello thoracis ofjeo, an itaque Labris anmunerandus vel Gafterofteis? Spina prima torfi antrorfun decumbens fixa, vti in Scombris quibusdam.

Pungi- 5. G. fpinis dorfalibus decem. tius.

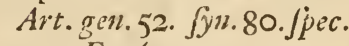
97. Fn. Juec. 277. Hubitat in Europa.

volitans. 6. G. fpinis dorfalibus tredecim, cirris fenis, pinnis pectoralibus corpore longioribus.

Gron. mul: 2. 11.19I. Perca dorfo monopterygio, capite cauernofo, muxilla fuperiore cirris 4 , cauda fubrotunda vtrinque aculeata. $\quad$ B.7.D.13-I2.P.I4. V.6. A. $\frac{1}{9} \cdot$ C.I3.

Will. icbt. app. r.t. 2. n.3.

Perca amboinenfis niehofii.

Ruyfch.thef. 1.p.39.t.2.f.3.

Valcnt anb. t.20. n. 210.

et t. $213 \cdot n .213$.

Habitat in Amboina.

Radii dorfales I3 priores bafi tantum inembrana connexi fint, et Jpino/i. P. pectorales corpore longiores yolantes. Cauda fipra et infia aculeis 3 minimis.

Spina- 7. G. fpinis dorfalibus quin-

chia. decim. Avt.gen. 52. Jyn. 81. Muf:Ad. Fr. 2. p. .. D. I5, 6.P.10.V. 0. A.7.C.I2. Habitat in Europa anjtraliore. 


Spinarel- 8. G. capitis poftico fpinis

la. quarernis ferrulatis longitudine abdominis.

Mirf. Ad. Fr. I. p. 74. t.32.

f. 5. Pungitius. D.I6. P.20.V.4. A. 8. C... Habitat in India.

146. SCOMBER. Caput compreffum, laeue. Minzbr. branch. radiis VII. Corpus laeue, linea laterali portice carinatum. Pinnac fpuriale faepius verfus caudam.

* Pinnulis Jpuriis diffinctis.

Scom- I. S. pinnulis V.

brus. Fn. finec. 287. Scomber

radiis pinnartim pectoralium 20 , dorfalium 12. Art. gen. 30. Jjn. 48. Jpec. 63. Scomber pinnulis 5 in extremo dorio, fpina breui ad anum. D.12,12.P.20.V.6.A.13.C..Gron. muf.r. n.8I. idem D.II,II. P.I8. V.6. A I2. C...Habitat in Oce:no Atlantico victitans Meduffs, vt congeneiv's.

Lucet in tenebris.

Pelamis. 2. S. pinnulis inferioribus VII, corpore lineis verinque quatuor nigris. Lnefl. epift.D. 15.II.P.27.V.6. A.14. C. .Osbeck. iter. 67. Scomber pulcher $r$ Bonet. Habitat in Pelago inter Tropicos.

Thyn- 3. S. pinnulis vtrinque VIII.

nus. Osbeck.iter.69. Scomber

albicans I. Albecor. D.I4,12.P.32.V.6.A.r3.C. jo. Art. gen.31. Jyn.49. Scomber pismulis 8 . $1: 9$. in extremo dorfo, fulco ad pinnas ventrales. D. I4, I4. P.34.V.6.A.I3.C... 
Loefl. epift. Scomber pinnulis 9, pinna dorí priori plicata, dentibus planis lanceolatis, maxilla fuperiore acuta. D.I4,I3.P.22.V.6.A.I6.C. .

E'roron. ian. 45 I. Thynnus pinnulis fuperioribus 9 , inferioribus 8 .

Habitat inier Tropicos, in Pclago.

Sociomm Garun pretiofa pifcium Sanies ex inteftinis pifcium falitis.

Cordyla. 4. S. pinnulis X, linea late. rali loricata.

Gron. Aft. VPS. $1750 . p \cdot 36$. Scomber linea laterali curua tabellis offeis loricata, corpore lato et tenui.D.7,9.P.15.V.6.A.2,I4.C.20: Marcgr. braf: I72. Guara tereba.

Rai. pifc. 93. Trachurus bialilientis.

Habirat in America.

* * Pinnulis Spuriis comatis.

Glaucus.5. S. pinnulis nullis, fpina dorfali recumbente.

Osbeck. iter.296. Scomber adfcenfionis.

Art.gent.32. (jn. 5i. Scom-

D.7,25.P.20.V.5.A.25.C.20。 ber dorfo dipterygio, offculo fecundo pinnae dorfalis altifimo.

D.7,26.P.18.V.5.A. $\frac{2}{27} \cdot C_{0} \cdot-$ Habitat ad inf. adfcenfionis.

Trachu- 6. S. pinnulis vnitis, fpina rus. dorfali recumbente, linea laterali loricata. Art. gen. 3r. Jyn.50. Scomber lines laterali aculeat2, pinna ani oficulorum 30. D.8,34.P.20. V.6.A. $\frac{2}{30} \cdot$ C. .. Mur. 


Muf: Ad. Fr. I.p.72. idem. D.8,31 P.20.V.6. A.27. C. 22.

Cloin Lagerftr. 27. Scom-

ber linea laterali fquamis

Igris pinnat:a.

Haffelqu. iter. 363 . Scom-

D.8,34.P.18. V.6.A. $\frac{2}{30} \cdot$ C. I7.

ber Trachurus.

Habitat in Mediterraneo.

Amia. 7. S. pinnulis vnitis, radio vltimo dorfalis pofterioris longiore.

Art. gुen. 3I. fyn. 5I. Scomber dorfo dipterygio, oficulo vltimo pinnae dorfalis fecundae praelongo. D.5,34.P.20. V.6.A.24.C.-. Habitat . .

pelagi- 8. S. pinnulis pinnaque dorcus. fali coadunatis in vnum. Mul: Ad. Fr. I. p. 72. Scomber pinna dorfali vnica. D. 40.P.19. V.5. A.22.C. 20. Habitat . . D. 8,32.P.20.V.6.A.30.C.2O. 
Seneca, Horatio, Imenali, Martiali; emebatur. olim argenti puri pondere, ideoque non edit Mullum, qui eum capit; maxime friabilis.

Surmu- 2. M. cirris geminis, lineis letus. luteis longitudinalibus. Art. gen. 43. Jyn.71. Trigla capite glabro, lineis vtrinque 4 luteis longitudinalibus.

Habitat in M. Mediterraneo et ad Cornubiam.

imberbis. $\hat{\jmath}$. M. imberbis.

Art.gen. 43. Jyn.71. Trigla

capite glabro, tota ru-

bens, cirris carens.

D.6, $\frac{\mathrm{r}}{\mathrm{I} 0} \cdot \mathrm{P} \cdot \mathrm{I2} \cdot \mathrm{V} \cdot 6 \cdot \mathrm{A} \cdot \frac{2}{10} \cdot \mathrm{C} \cdot 20$.

Habitat in Melita.

148. TRIGLA. Caput loricatum lineis fcabris. Membr. branch.. radiis VII. Corpus: Digiti liberi ad pinnas pectorales.

cataphra- I. T. digitis geminis, roftro

Eta. . furcato elongato, corpore loricato. $\quad$ B.7. D.27.P.II. V.6.A.20.C.IO. Art.gen. 46. Jyn. 75. Trigla cirris plurimis, corpore oktagono. Gron.muf.r.n.98. idem.B.5. Habitat in M. Mediterraneo: fupero inferoque.

Pinnae ventrales adnexae pectoralibus. Cirri pluves ád labian inferius. Os edentulum.

Lyra. 2. T. digitis ternis, naribus tubulofis.

Art. gen. 46. fyn.74. Trigla roftro longo diacantho, naribus tubulofis.

Habitat in Mari Britannico.

D. 10, 18.P.12. V. $\frac{1}{6} \cdot$ A. - C. . - 


Gurnar- 3. T. digitis temis, dorfo

dus. maculis nigris rubrisque. D.9,19. P.IO. V.6.A.19.C.I5. Art.gen. 46.jyn.74. Trigla varia roftro dracantio, aculeis geminis ad vtrumque oculum.

Gron. mu!. I. n.I01. idem. D.9.I8.P.10. V.6.A.17. C.I5. Iiabitat in Oceano Britannico.

Cuculus. 4. T. digitis ternis, linea laterali mutica. Art.gen. 45. (jnz.74. Trigla

D.9, I6.P.I0.V. T. A.I6.C.I3. toca rubens, roftro parum bicorni, operculis branchiarum friatis. D.9,17.P.II.V.6.A.I5.C.I3 Habitat in Mari Mediterraneo, Pelago.

Lucerna. 5. T. digitis ternis, roftro fubbifido, linea latcrali ad caudam bifida.

Art.geir. 45. fyn.73. Trigla roitro parum bifido, linea laterali ad caudam bifurc: D.10,17.P.?0.V.6.A.15.C. . Gron. mul: I. $n$ Ioo. idem. D.8, 16. P:10. V.6.A.15. C. = Habitat in Oceano jepteritrionali.

Hirundo. 6. T. digitis ternis, linea 1aterali aculeata.

Avt.gen. 44.Jyn.73. Trigla capite acule ato, appendicibus vrrinque tribus ad pinnas peetorales. Fn. fuec. 28 I. It. Wgot. p. I76. idem. D.7,I9.P.9.V.6.A.IS. C.II. Habitat in Oceano Atlantico.

D.9,I8.B. I0.V. $\frac{\pi}{6}$. A. I9. C. I2. Pinnue pectorales aigrae.

afiatica. 7. T. digitis quaternis. B.7.D. $\frac{1}{3}, 16 . P .19 . V .6 . A .17 . C . I S$. Habirat in Oceano afiatica. 


\section{PISCES THORACICI. Trigla.}

Corpus laeue, teretiufculum, argenteum. Nafus f. rofrum pronbinens, laeue. Os intus fabrumb. Opercula anteviora firrata. Pinnae pectorales falcatae.

volitans. 8. T. digitis vicenis palmatis.

Art. gen. 44. Jyn. 73. Trigla capite parum aculeato, pinnula fingulari ad pinnas pectorales.

D. 5, 8. P.28. V.5. A.6. C. .. Gron. muf.r. n. 102. idem. D.6,8. P.6. V.5. A.6.C.Is. Habitat in Mari Mediterraneo, Oceano, Pelago inter. tropicus, in Afia, imprimis ad Cap. b. fpei, faepe agitata euolans ex aqua. 
Trigla abseura L. 1764. lo. A. F. p.gh. 



\section{ABDOMINALES.}

149. COBITIS, Caput: Oculi in fuprema capitis parte.
Membr. brancb. radiis IV-VI. O. percula inferne claufa.
Corpus fere aequale 1 . Cauda verfus pinnam minus anguftata.

Anz- I. C. capite inermi ciepreffo;

bleps. oculis prominulis. Art. gen. 25. Anabieps.

Gron. mni: I. n. 32. t. I. f. I, 2, 3. Anableps. B.6.D.7.P.22.V.7.A.9.C... Habitat in India.

Burbatu- 2. C. cirris oris 6 , capite inla. ermi compreflo. Art.gen. 2.jyn. 2. Cobitis tota glabta maculofa, cor$\begin{array}{ll}\text { pore lubtereti. } & \text { D.8. P. 12. V. 7. A.6. C. . }\end{array}$ Faun. Juec. 332. idem.

Habitat in Europae, Afiae aquis dulcibus.

Taenia. 3. C. cirris oris 6, fpina fuboculari. Art. gent. 2. $\int y n \cdot 3 \cdot \int p c c .4$. Cobitis aculeo bifurco infin verumque oculum. D.9. P.7. V.7. A. 8. C. IS. Fin. jucc. 333. idem.

Grom. mu!: 1. 11. 5. idem. D.7. P..- V.7. A.6. C. 16. Habitat in Europae aquis dulcibirs.

forfilis. 4. C. cirris otis 8 , fpina fus pra-oculari.

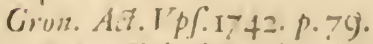 t. 3. Cobrtis aculeo hi- furco fupra vertunque $n$ - culum. B.3.D.7. I.9. V.6.A.7.C. 16. Art. gen. 2. Jyn. 3. Co- bitis caerulelcens, lineis


vtrinque 5 longitudinali-

bus. $\quad$ D. 7.P. II. V.5. A. 5. C.16. Muf. Ad.Fr. r.p.76.idem. D.6. P. II. V.6. A.6. C. I5\% Habitut in Europa.

150. SILVRVS. Caput nudum. Os cirris aliquot filiformibus tentaculatum. Membr. branch. radiis IV - XIV. Corpus: Radius pinnarum pectoralium aut dorfalis primus fpinofus, retrodentatus.

Afotus. I. S. pinna dorfali vnica, cirris 4 . Habitat in Afia. M. Ac. Holmenfis.

Cirri duo Supra os; duo infra. Dentes mameroff. Spina mulla rigida in pima dorfi, at ferrata in Pectoralibus, vt in congeneribus. P. ani longa, caudae annexa.

Glanis. 2. S. pinna dorfali vnica, cirris 6.

It. jcan. 6r. Mal. B.I4.D.4.P. $\frac{T}{18} \cdot$ V.12.A.8S. C. 17 . Act. Stockh. 1756 . p. 34 .

t. 3 . Silurus. B.16. D.4.P. $\frac{1}{17} \cdot V \cdot 13 \cdot A \cdot 88 \cdot$ C.17. Art. gen. 82. Syn. no. Silurus cirris 4 in mento.

Gron. muf. 1. n. 25. SiluD. 4.P. $\frac{1}{16} \cdot$ V.II. A.20. C.17. rus cirris 2 ad maxillam fuperiorem, 4 in mento.

$$
\text { B.I4. D. I. P. } \frac{T}{15} \cdot V \cdot \text { II. A. 90. C. I5. }
$$

Habitat in Oriente, minus frequens in Europae lacubus. Maximus dulcium; iudaeis vetitus.

Afpredo. 3. S. pinna dorfali vnica: radiis 5 , cirris 8 . Anuen. acud. I. p. 3 Ir. $t$.

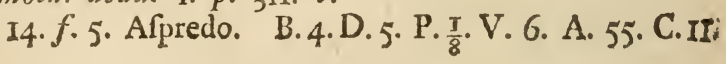






\section{PISCES ABDOMINALES. Silurus. 305}

Gron.muf. 1. n.26. Afpre-

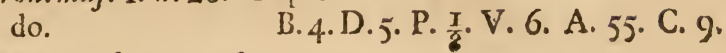
Klein. miff. 5. p. 86. t. 4 . f. 8. Batrachus.

Habitat in Americae flumiis.

Mýnus. 4. S. pinna dorfali vnica, radiis 6, cirris 8. Musf. Ad. Fr. 2. p... $\quad$ B. . D. D. $\frac{x}{5} \cdot$ P. $\frac{x}{9} \cdot$ V.6.A. $57 \cdot$ C. $19:$ Hajelqn. iter. 376 . Silurus Schilbe niloticus. B.rO.D. T. P. I $\frac{1}{1} \cdot V \cdot 6 \cdot A \cdot 62 \cdot C \cdot 20$. Habitat in Nilo.

anguilla- 5. S. pinna dorfali vnica, ra-

ris. $\quad \operatorname{diis} 70$, cirris $8 . M u$. Ad.

Fr. 2. p... $\quad$ B. .. D.70. P. $\frac{1}{7} \cdot$ V.6. A. 55. C. 17. Hafjeliqu. iter. 37r. Silurus

Charmuth niloticus. B.1O.D.72. P. $\frac{T}{10}$. V.6.A. 59. C. 2 I. Habitat in Nilo.

Batra- 6. S. pinna dorfali vnica rachus. diis 60 , cirris $8 . M u$. Ad. Fr. 2. p... B.5. D.60. P. $\frac{\pi}{8}$. V.6. A. 48. C. I4. Ilabitat in Alia, Africa.

vndeci- 7. S. pinna dorfali vnica ramalis. diis II, cirris \&. Muf. Ad. Fir. 2. p... B. - D. D. $\frac{\mathrm{I}}{\mathrm{II}} \cdot$ P. $\frac{\mathrm{I}}{\mathrm{II}} \cdot \mathrm{V} \cdot$.6.A.11. C.17. Habitat . .

militaris. 8. S. pinna doffali poftica adipofa, cirris 2 rigudis. B.--D. $\frac{1}{7}, 0 . P \cdot \frac{1}{I I} \cdot V \cdot 7 \cdot A \cdot 20 . C . I 8$. Habitat in Afia. M. Ac. Hulmenf.

Oculi ad latera capitis. Cirri 2 ad os, compreffi, rigidi, olfei. Linea lateralis recta. Dorfum ad pinnam dorji "Ifurgens.

Catus. 9. S. pinna dorfali poftica adipofa, ani radiis 20 , cir-

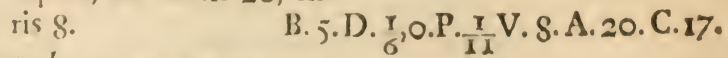

Catcsb car. 2. p. 23. t. 23 . bagre a Marcgr. affinis. 


\section{PISCES ÁBDOMINALES. Silurus.}

Marcgr: braf. 173. Bagre fiecies 2.

Habitat in America, Afia. Ex Ajia vidi pinnis :entr. radiis 6 ,

Clarias, Io. S. pinna dorfali poftica adino $\lfloor$, ani radiis $\mathrm{II}$, cirris 6 . Muj: Ad. Fr. 1. p. 73. Afpredo ninna dorlali pofica adipofa. $\quad$ E.9.D. $\frac{1}{7}, 0 . P \cdot \frac{1}{10} \cdot V \cdot 7 \cdot A \cdot I 1 \cdot C \cdot 17$. It. fcan. 82. Afpredo cirris Ion gitudine corporis, pinnis dorfailbus duabus. $\quad$ D. - D.7,0.P. $\frac{1}{9} \cdot V \cdot 5 \cdot A \cdot 10 . C \cdot 16$, G;0n. mu..r. n. 83. Myftus cirtis 6 long firmis, pinna dorfi fecunda longiffina a priori ad caudim extenfa.

Halfelequ. iter. 369 . Silurus Sicheilan niloticus. B.6. D. $\frac{1}{7}$, O.P. $\frac{1}{9}$. V.7.A.12. C.16. Habitat in Americae, Africae fiutiis. Ir. Nilo vencuatus radio dentuto pectoraliun.

Afcita: II. S. pinna dorfali poftica adipofa, ani radiis 18 , cirvis 6.

Mil" Ad. Fr. I. p. 79. t. 30, f. 2. Alcita. B. -. D. $\frac{1}{8}, 0 . P \cdot \frac{1}{12}, V \cdot 6 . A \cdot 18 \cdot$ C.I8.

Hahitat in India.

Ouis muturis, intumefcente abdomine, longitudinaliter. debilicit.

coftatus, I2, S. pinna dorfali poftica adipof, fquamis ferie fimplici, cirris 6 , cauda bifida.

Gron. $\operatorname{muf} 2 \cdot n \cdot 177 \cdot t \cdot 5 \cdot f$. I. 2. Myitus cirris 6 iongifimis, vaico fquamarum acuicatarum ordine viringla in lareribus. D. $\frac{1}{7}, 0 . P \cdot \frac{x}{9} \cdot V \cdot 7 \cdot A \cdot 12 . C \cdot 17$. Hubitat in Indiis. 


Callich- 13. S. pinna dorfali poftica thys. vniradiata, fquamis ordine duplici, cirris 4 .

Amoen. acad. I. p. 317. t. I4. f. I. Callichthys. B. 3.D. I I I. P. T. V. G.A. $\frac{1}{7} \cdot$ C. 14 . Gron. mul. x. n. 70. Callichthys cirris 4 , laterihus duplici fquamarum ordine. B.3.D. $\frac{2}{8}, I \cdot P \cdot \frac{1}{17} \cdot V \cdot 7 \cdot A \cdot 7 \cdot$ C. 14 .

Barrer. acquin. 175. Mullus palultris minor loricatus.

Habitat in Americae riuulis. Riunlis exficcatis exit per terram aquas quaefiturus. Marcgr. braj: $15 \mathbf{1}$.

cata- 14. S. pinna dorfali poftica vphrątus. niradiata, fquamis ordine fimplici, cirris 6, cauda integra.

Gron. mul. I. n. 71. t. 3. f. 4. 5. Callichthys cirris 6 , lateribus vno fqua-

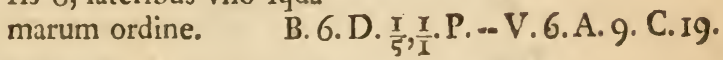

Catesb.car.3. p. 19. t. 19. Cataphractus americanus. Habitat in America.

I5 I LORICARIA. Caput laeue, depreffum. Os edentulum, retractile. Membr. brancilo. radiis VI. Corpus cataphractum.

cataphra- x. LORI CAR a Mul. Ad. Fr.

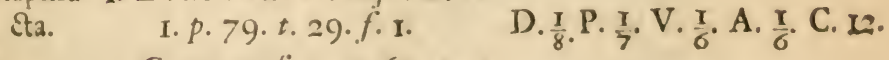
Gron. wirl: I. n. 68. t. 2. f. I. 2. Plecoftomus dorfo monopterygio, ofliculo fuperiori caudae bifurcae fetiformi. D. $\frac{1}{8} \cdot$ P. $\frac{T}{3} \cdot$ V. $\frac{\pi}{6} \cdot$ A. 6. C. 12 . B. Grons. 
B. Gron. muf. I. n. 69. Plecoftomus ore edentulo, oficulo fuperiore caudae bifurcae longitudine corporis.

Habitat in America meridionali.

Lobus superior caudae filo longiffino terminatur.

152. SALMO. Cotput lacue. Dentes in maxillis, lingua.

Menibr. branch. radiis IV -X.

Corpus: Pinna dorfalis poftica adipofa; Pinnae ventrales multiràdiatae.

* trvttae corpore variegato.

Salar-. x. S. roftro vitra inferiorem maxillam prominente. Art. gen. II. Jin. 22. Spec. 48. Fn. Jisec. 306. B. 12.D.15.P.14.V.ro. A.13.C.19.

Habitat in Oceano Europae, parit in fluniis.

Mas maxilla fipperiore adunca; fortiter flumios adfcerdit; allicitur albo.

Erinx. 2 2. S. maculis cinereis, caudae extremo aequali. Art. gen. 12. (jn. 23. Spec. 50 . Fn. fiuec. 307 .

Hahitat cum pracedente.

Trutta. 3. S. ncellis nigris iridibus brunneis, pinna pef̃orali punctis 6. Fin. jiuec. 308. $\quad$ B.-D.I2.P.13.V.10.A. 9.C. 20. Ait. gen. 12. fyn. I4. Salmo latus, maculis rubris nigrisque, cauda aequali. Gron. m:1. 2. n. 164. Sal-

.mo latus, cauda fubrecta 


maxillis aequalibus, ma-

culis nigris annulo albido. D. I4.P. I2. V. I2-A. IO. C. Habitat in flumiis Europae.

Fario. 4. S. maculis rubris, maxilla inferiore fublongiore.

Ari. gen. 12. Jyn. 23.

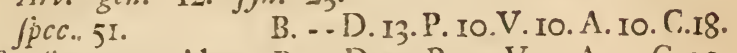

Fn. Juec. 309. idem. B.ro.D.14.P.14. V.9. A. I. C. 20. Habitat in Sueciae, Heluetiae flumiis.

Hucho. 5. S. oblongus, dentium lineis duabus paiati, maculis tanrummodo nigris. Art. gen. 12. Jyn. 25.

Habitat . .

lacuftris. 6. S. cauda bifurca, maculis folum nigris, fulco longitudinali ventris. Art. gen. 12. Jyn. 25.

Habitat in Heluetia.

Carpio. 7. S. pede minor, dentium ordinibus quinque palati. A; t. gein. Iว. Jyn. 27 .

Habitat in Angliae, Vallefiae flutiis.

alpinus. S. S. dorfo nigro, lateribus caeruleis, veritre fuluo. Fin. Suic. 310.

It.Wgot.257. Roeding.E.10. D. 13. P. I4.V.10. A. 12.C.I9. Art. gen. 13. Jjn. 25. Jpec. 52. Salmo vix pedulis, pinnis venrris rubris, maxilla inferiore paulo longiore.

Habitat in Lapponiae, Angliae alpibus, folus. Caro rubra.

Salueli- 9. S. pedalis, maxilla funenus. riore longiore. Art. gens. 13. Ijn. 26.

Habitat in Aultria ad Lintz. 
Salmari- IO. S. dorlo fuluo: maculis

nus. luteis, cauda bifurcata. Ant. $\int y n .24$.

Habitat Tridenti in flutiis frigidis faxofis.

Vmbla. II. S. lineis lateralibus furfum recuruis, cauda bifurca. Art. gen. 13. $\int y n$. 25.

Habitat in Heluetiae, Italiae lacubus.

* osmer I pinna dorfali anique oppofitis.

Eperla- 12. S. capite diaphano, ra-

nus. diis pinnae ani $I 7$.

Art.gen. 10. $\int y n$. 2I. $\int p e c$.

Fn. jiec. 3 Ir. Ofmerus ra-

D.II.P.Ir. V.8. A. 7. C. 19.

diis pinnae ani 17.

Habitat in Europa, flmios primo vere adfcendens.

Nofter foetidus prurit cum Salice rubra et Viola odorata.

Saurus. 13. S. radiis pinnae ani ro.

Art. gen. 10. fyn. 22 . O.

fmerus radis pinnae ani

Io.

Habitat in Europa.

B. -- D. 12.P.13. V.8. A.10, C.-.

* * C CREGON I Dentibus vix confpicuis.

Lauare- If. S. maxilla fuperiore lontus. . giore, radiis pinnae dorii 14 .

Art. gen. 10. $\int y n$. 19. $\int p e c$. 37. Coregonus maxilla fuperiore longiore, pinna doiti osficulorum I4. Fn. litec. 322.

Ait. Stockh. 1753. p. 195 . Habitat in Europa.

Albula, I5. S. maxillis edentulis: infcriore longiore.

Art. gen. 9. Jyn. 13. Jpec. 40. Coregonus edentu- 


lus, maxilla inferiore lon-

giore. Fin.fute.313. B.7.D. 14. P.16.V.12. A.15.C...

Habitat in Europa.

Prurit ante Lrwnam.

Thymal- 16. S. maxilla fuperiore lon-

lus. giore, pinna dorfi tadiis 23 .

Art. gen. 10. Jyn. 20. Jipe. 41. Coregonus maxilla fuperiore longiore, pinna dorli officulorum 23 .

Fin. Jitec. 3is.. idem. B. IO. D.23.P. I6.V.12. A. I4.C.Ig. Gron. mul. 2. 11. 165. idem. B... D.2I. P.15. V.IO.A.15.C... Oxyrinohus Habitat in Europae flutiis nizaritimis.

Oxyrhyn-17. S. maxilla fuperiore lon-

clus. giore conica.

Art. gen. 10. Jỳn. 2I. Coregonus mixilla fuperio-

re longiore conica. B. - - D.13.P.17.V.I2. A.I4.C. -

Grone. mut: I. n. 48 . i-

dem. $\quad$ B.--D.I4.P.I3.V.IO. A.I5. C.--

Habitat in Oceano Atlantico.

Vimba. Is. S. pinna adipofa fubferrata.

It. It gut.p. 231 IV Imba. B. - - D.I2.P. I6.V.IO.A.I4.C.-Habitat in Waencro Suecise.

* * * characina Membr. branch. radiis tantumIV.

gibbofus. 19. S. dorfo gibbofo compteffo, pinna ani radiis jo. Gron. mulf. I. n. 53. t. I. f. 4. Charax dorfo admodum prominulo, pinna ani radis 55: D.IO.P.II.V.S. A.55. C. 19.

Habitat Surinani.

bimacu- 20. S. corpore compreffo hilatus. macuiute, pinna ani iadiis 30 .

Grons. 
Gront. mul: I. n. 54. t. I. f. 5. Cinrax dorio leuirer conuexo, pinna ani radis 3 i.

Mul. Ad. Fr. r. p. 78 . t.

D. I0. P.I3. V. 8.A. วั. C.I9. 32. $t .2$. Albula pinna ani radiis 32 . B. 4. D. IO. P. I3. V.8. A.32. C.I9. Art. Jpec. I4. Coregronoides amboinenis.

Habitat in America meridionali.

immacu- 2I. S. corpore immaculato,

latus. pinna ani rudiis 12.

Mif. Ad. Ir. I. p. 78. Albula pinna ani radiis I2. D.Ir.P.I4.V.II. A.12. C. 20. Ilabitat in America.

niloticus. 22. S. pinnis omnibus flauefentibus, corpore toto albo. $\quad$ D.9.P.13. V.9. A.26. C.19. Habitat in Nilo.

pulueru- 23. S. pinnis fubpuluerulentis, lentus. $\quad$ inea laterali defcendente.D. Ir. P.ró. V.8.A.26. C. I8. Habitat in America.

anofto- 24. $S$ ore furfum fpectante. mus. Gron. mul:2.n. 165.7 .7 . f. 2. Anoltomus. B.4. D.rI. P.13. V.7. A. I0. C. 25 . Habitat in Indiis.

153. FISTVLARIA. Caput: Roftrum cylindricum apice maxillofum.
Monubr. brancis. raciiis VII.
Corpus ...

Cabacaria. I. Fistrinia. Muf. Ad. Fi. I. 1). 30. t.26.f.I. D.I4.P.I4.V.6. A. 4. C.I3. Gron. mul: I. n. 3I. Solenoftomuc ciuda bifurca in ietam buldenaceam abeunte. D. S. P.15.V.6. A. 12.C.12.

Catesb. 


Catesb. car. 3. t. I7. f. 2. Petimbuaba. Marcgribraf. 148.

Will. iclst. app. 22. Peten-

Hubitat in America.

Filum e diviju a pinnae caudae.

154. ESOX. Coput fupra pleniufculum: Mandibula fuperiore plana bretiore: infriore punctata. Dentes in maxillis, lingua.

Menlir. branch. radiis VIII-XIII.

Corpus elongatum.

Sphyrac- I. E. dorfo dipterygio : anti-

na. ca fpinofa. D. 5.I0.P.12. V.6.A. Io.C.17. Ait. gen. 84. Jyn. 112. Sphyraena.

D. 5, IO.P.I3. V. 6. A.IO. C. . -

Habitat in Mari Mediterraneo.

offcus. 2. E. pinna ani duplici, fquamis of?eis.

Art. gen. I4. Syn. 27. E-

D.6. P.II. V. 6. A. 5. C.I2. fox maxilia fuptriore iongiore, cauda qua drata.

Gron. muf I. "1.29. idem. Catesb. car: 2.t. 30. Acus $\mathrm{m} \cdot \mathrm{x}_{1} \mathrm{ma}$ fquamofa viridis.

Hlibitut in Anerica feptentrionali, Afia.

Vulpes. 3. E. pinna in medio dorfi, membrana branchuoltega triradiuta.

Catesb. car: $=$. t. I. $f \cdot 2$. D. 14.P. I4.V.S.A.10. C. 17 . Vulpes bahamentis.

Habirat in America.

Synodus. 4. E. pinna in medio dorfi, membran lirancuiofiegra

5-radians.

Ciron. met: 2, n. 151. t. .

f. I. Synodus. L. ..D. II.P.I2.V.S. A.6.C.S. 


\section{PISCES ABDOMINALES. Efox.}

Habitat in America.

Sinnilitudo Jumna cum E. Vulpe.

Lucius. 5. E. roftro deprefio fubaequali.

Ar*. gen. 10. $\int y n$. 26. $\int p e c$. 53. Fn. Juec. 304. Efox roftro plagioplateo. Gron. muf. I. n. 28.iden. D. I8. P. II. V. 9.A. 15. C... Habitat in Europa.

Voraciffinus exbaurit pifcinas; ab Anatibus feritur.

Hepfe- 6. E. linea laterali argentea.

tus.

Annon. acad. 1. p.321. Argentina pinna dortili pinnae ani oppolita. D.I4.P.I2.V.6.A.15.C.14.

Marcgr.braf. I59. Piquitinga.

Brown. iam. $44 \mathrm{r} \cdot \mathrm{t} \cdot 45 \cdot \mathrm{f}$. 3. Menidia corpore fubpeliucido, linea laterali latiori argentea.

Hobitat in America.

Dinerfics ab Hepfeto Atberinae pimna dorgle unica, et maxilla fuperiore etc.

Bellone. 7. D. roftro vtraque maxile la fubulato.

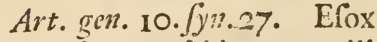
roftro cufpidato gracili fubtereti fpirhamali.

Fn. fuec. 305. julem.

Gron. mij: I. 21. 30. i-

D. 16. P.13.V.7.A.20.C... dem. B.I4.D.16.P.13.V.7. A.21. C. 23. Habitat in Oceano Europaeo. Ofja noctu lucest viridia.

brafilien- 8. F. maxilla inferiore lonfis.

s. gifima, corpore ferpertino.

D.I2.P.10.V.6.A.17.C.16.

Marcgr.braf 16s. Timucu.

Bromn. iann. 4.43 t. $45 . f .2$.

Efox maxilla inferiore

producta.

Habitat in America aufroali. 


symno- 9. E. maxillis aequalibus, o-

cephalus. perculis obtulifimis, capite denudato. $\quad$ D.13.P.I0.V.7.A.26.C. I9.

Habitat in India.

Magnitudine Ammodytis qui nobis vifus.

155. ARGENTINA. Corpus: Dentes in maxillis, lingua.

Membr. branch. radiis VIII.

Corjus ano caudae vicino. Pinmate ventrales multiradiatac.

Sphyrae- I. ARGENTINA. Art.gen.S.

nia.

Gron. 17.

tina.

Habitat in Mari infero.

Veficula aïrea vtrinque conica velut argento foliato rejplendens, vnde margaritue artificiales. Rai.pijć. 10s.

D. 10. P.14.V.II. A. 9. C...

D. I0. P... V... A.10. C...

156. ATHERINA. Caput maxilla fuperiore planiufcula. Mandibulac edentulae. Membr.branch. radiis VI. Corpus fafcia laterali argentea.

Hepfetus. I. ATHERINA.

Arted. Jyn. II7. Atherina.
Gron. mul: I. n. 66. Athe-

Arted. $\int y n$. II 7 . Atherina.
Gron. mur. I. n. 66. Atherina.

Haffelqu. iter: 382. Athe-

D. S, 12.P.13.V. T. A.I6.C.17. rina Hepfetus.

Habitat in Mari Mediterraneo.

D.6,12.P. 13.V.6. A.IO.C. .D. S. 9. P. I2. V.6.A. ${ }_{-1}^{T}$. C.20. 
316 PISCES ABDOMINALES. Mugil.

157. MVGIL. Caput: Labia membranacea: inferius introrfum carinatum. Dentes nulli. Denticulus infexus fupra finus oris.

Membr: branch. radiis VII curuis; Opercula laeuia rotundata. Corpines albicans.

Cepha- I. MVGIL. Jus Art.gen. 32. Jyn. 52. Spec.

7 r. Nugil.

Hafelqu. iter. 385. Mugil

D. $4, \frac{I}{2} \cdot P \cdot 16 \cdot V \cdot \frac{T}{6} \cdot A \cdot \frac{2}{12} \cdot C \cdot 12$.

Cephalus.

D. $5, \frac{I}{11} \cdot$ P.I8.V. $\frac{I}{6} \cdot A \cdot \frac{3}{I^{3}} \cdot C . \cdots$

D. 5, II. P. 13.V. 6. A.10.C. -.. Habitat in Oceano Europaeo, flumios fibiens. Botargo itaionun ex buius ouis.

158. EXOCOETVS. Ciput fquamofum. Os edentulum: maxillis vtroque latere connexis.

Menbr. branch. radiis X.

Corpus albicans. Abdomen angulatum. Pinnae pectorales : maximae, volatiles radiis antice carinatis. .

volitans. I. E X O C O E T V S. Amoen.

acad.1. p.321.

Art. gcu. 8. Jyn.18. Jpec.35.

Exocoetus.

Gron. nuf. I. n. 27. Exo-

D. 14.P.15. V.6. A.13. C.15. coetus.

D. I3. P. 17. V. 6. A. II. C.15.

D.12. P. 17. V. 7. A... C. .. Habitat in alto Pelago, Europaeo et Americano, a Coryphaenis exagitatus ad fclopeti iactum ex aquis eurlans gregarius, praeda fit Aquilae Pbaëtontis; an Selaw Ifruelitarunn, iubente Auciore coryphaenas pellere bos ad litius, vit Jtatuit Rudbeck. fil.? 

159. POLYNEMVS. Caput compreffum, vndique fquamofum: roftro obtufiffimo, prominente.

Membr. branch. vadiis VII. . V. Corpus Digitis liberis ad pinnas pectorales.

quinqua- I. P. digitis quinque corpore

rius. longioribus.

Gron. muf: I. n. 74. Polynemus officulis filiformibus verinque 5 ad pinnas pectorales.

Habitat in America.

B. 5.D.7,16.P.16.V. $\frac{1}{6} \cdot A \cdot \frac{2}{25} \cdot(\cdot 17 \cdot$

virgini- 2. P. digitis feptem, cauda

cus. integra.

Habitat in America.

B.7.D. $7, \frac{1}{15} \cdot$ P.15.V.1 $\frac{1}{5} \cdot A \cdot \frac{2}{15} \cdot C \cdot 15 \cdot$

Opercula jerrata. Radius primus R. dorfalis prioris breuis. fimus. Pinna caudae lata, acuta.

paradi- 3. P. digitis feptem, cauda

feus.

\section{bitida.}

Edro. au. 208. t. 208. Paradifea pifcis.

Habitat in India.

Genus a Trigla diftinctun pinnis ventralibus, non in pecture, fed abdomini fitis; nec digiti articulati, vt in Tiglis.

160. CLVPEA. Caput maxillarum fupcriorum myftacibus ferratis.

Membr. brancb. radiis VIII: Branchiae interne fetaceae.

Corpus: Albdominis carina ferrata. Pinnate ventrales fiepe nouemradiatae.

Haren- I. C. immaculata, naxilla

gus. inferiore lungiore. D. I8.P.1S.V.9. A.17. C.19. 


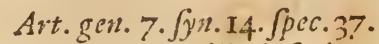

Clupea maxilla inferiore

longiore, maculis nigris

carens. $\quad$ D.I8.P.18. V.9. A.17. C.I8.

In. fuec. 3r5. idem.

Grun.muf I. n.21. iden. D.19. P.15. V.8. A.16.C. Habitat is M. Europaeis.

Europacis borealibus copiofiffinus pifcis.

Sprattus. 2. C. pinna dorfali radiis XIII. D. I7.P.I6.V.6.A.19.C... Art. gen. 7. Jyn. 17. Jpec.

33. Clupea quadruncia-

lis, maxilla inferiore longiore, ventre acutilimo. Fn. firec. 316.

Habitat in Oceano Europaeo.

Alofa. - 3. C. lateribus nigro macula tis, roftro bifido. Art. gen. 7. Jyn. 15. Jpec.

D. 19.P.15.V.9.A.21.C. 19. 34. Siupea apice maxillae fuperioris bifido, maculis nigris vtrinque. Gron.mif.1. n. 23. idem. D. I8. P. . V.8. A.20. C. Hafelqu. itin. 38s. idem. D. 19. P. I5. V... A. 18. C. 26. Habitat in Oceano Europaeo.

Encrafi- 4. C. maxilla fuperiore loncolus. giore.

Art. gen. 7. Jyn. 17. Clupea maxilla fuperiore longiore.

Habitat in Oceano Europaeo.

E culina expulit Garun veterun.

Thriffa. 5. C. pinna ani radiis 28 , dorfalis radio poftremo fetaceo longo.

Brown. iam. 443. Clupea minor, radio vltimo pinnae dorfalis longiffimo.

Chin. Lagerffr. 30. Myftus

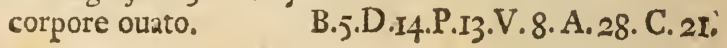




Osbeck. itin. 257. Clupea
Thriffa.

Hiabitat in Mari Indico.

Pinnae ventralcs fint paruae.

Sima. 6. C. pinnis flauis: ventralibus minutifimis. $\quad$ B.6.D.17.P. $\frac{1}{17} \cdot V \cdot \frac{1}{6} \cdot A \cdot 53 \cdot$ C. Is. Hubitat in Alia. Muf. acad. Holmens:

Os fimun: maxilla fiperime brevifina, upice truncata. Corpus argentenm. Abdomen carinato-jerratum. Lacuna inter oculos. Pinine flauae: ventrales adco par. uae, vt vix conjpicuae.

Sterni- 7: C. pinnis ventralibus nul-

cla. lis, B.2.D.II.P.9.V.0.A.34.C.IS. Gron. muf. 2. n. I55. t. 7 .

f. 5. Gafternpelecus. D.12.P.9.V.0.A.33.C.22. Habitat Surinami.

Corpus latifinnm, abgomine gibbofo carinatum.

Myftus. 8. C. corpore enfiformi, pinna ani caudue coadunata. D.I2. P. I8. V.6.A.84. C.II. Osbeck. itin. 256 . Clupea Myftus.

Chin. Lagerftr. 3I. t. I. f.

12. Niyfus corpore enliformi. D. I2. P.17.V.6.A.84. C.II. Habiat in Mari Indico.

Pinna ani excurrit in pimanu caudae integram; forte genere Jub duer-jo comprebendi pojJet?

tropica. 9. C. cauda cuneiformi. Osbeck. itin. $300 . \quad$ B.7.D.26.P.6. V.6.A.26.C. 20. Habitat ad inlallam Adfcentionis.

Corpus abum cumprefJum, latum, ferratum. Linea lateralis reefa, dorji propior. Oculi prope rifum. Opercula fiquan'yia. Ciput decliuc. Max:lla infirior longior. Ricius magnus. Dentes anius urdinis. Cauda cuncifirmis. P'. dorti a mediv dorja ad casdam. P. Ani longitudine dorjolis.

finenfis. 10. C. radio extimo $\mathrm{mem}$ branae branchoflegae pofirice in!ncatu.

D.16.P.16. V.S.A.16.C... llabirat in China. 
Similis Clup. Harengo, fed latior. Os edentulum. Opercula infina membr. branchiofl. tegentia valde thuncata f: potius radium extmmun inembr. brancbioftegae conftituentia.

16r. CYPRINVS. Caput ore edentulo; Os nafale bifulcum.

Menubr. branch. radiis III.

Corpus laeue, albens. Pinnae ventrales faepe nouemradiatae.

* Barbati f. ad os cirroji.

Barbus. I. C. pinna ani radiis 7 , cirris 4 , pinnae dorfi radio fecundo verinque ferrato. D. $\frac{2}{1 \mathrm{I}}$ P. I6. V.9. A.7.C. 16. Art. gen. 4. Jyn. 8. Сyprinus maxilla fuperiore longiore, cirris 4, pinna ani officulortm $\%$

Gron. muf: 1. 21.20. idem. D. $\frac{2}{12}$ P.I6.V.9.A.7. C.I6. muf.2. p. 3. idem. D. I0. P. 17.V.9. A. 8. C.17. Habitat in Europa aufrali.

Oua choleran cauffantui. Tim. caj: 104.

Carpio. 2. C. pinna ani radiis 9, cirris 4, pinnae dorfalis radio fecundo poftice ferrato. D. $\frac{2}{24}$ P. 16.V.9. A.9. C. I9. Art. gen. 4. Jyn. 3. Jpec. 25. Cyprinus cirris 4. osficulo tertio pinnarum dorli anique vncinulis armato.

Fn. fuec. 317. idem.

Gron. muf. I. n.19. idem. D.23.P.17.V.S. A.8. C. -Habitat in Europa.

Nobilis pifcis faepius in pifcinis educatus, circa a. 1600 primum in Anglian introductus.

Gobio. 3. C. pinna ani radiis II, cirris 2.

D.10.P.16.V.g.A.II. C.19. 




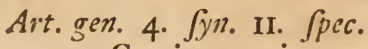

13. Cyprinus quincuncialis maculofus, maxilla fuperiore longiore, cirris duobus ad os.

Gron. mus. 2. 12. 149. iD.I2.P.I7. V.II. A.Ir. C.I9. dem?

Habitat in Anglia et adiacentibus.

america - 4. C. pinna ani radiis .., cirnus: ris plurimis.

Catesb. car. 2. p. 12. t. 12 . Alburnus americanus.

Habitat in America.

* Pinna caudae indinija.

Caras- 5. C. pinna ani radiis 10, fius. caudae integra, linea la$\begin{array}{ll}\text { terali reßta. } & \text { D.20. P.I5. V.8. A.9. C. } 2 \text { I. }\end{array}$ Art. gent. 4. Jyn.5. Jpec. 29 . Cyprinus pinna dorfi osficulis 20 , linea laterali reßta.

Fn. Jitec. 322. idem. D.20.P.15. V.9. A.10. C. :Gron. muS I. n.II. idem. D.20.P.II. V.9. A. IO. C. 23 . ACt. VPJ: 1741. p. 75. $n$.

55. (yprinus Hamburger. D. 20.P. I4. V. 9. A. 9. C. 22. Habitat in Europae ftagnis.

Facilline in pifcinis educatur.

Tinca. 6. C. pinna anni radiis 25 , caudie integra, corpore mucofo.

Art. gent. 4. Syn. 5. Spec.

D. 10.P. 16.V.9.A.25.C. 24 .

27. Cyprinus mucofus

totus nigrefcens, extre-

mitate caudae aequali. D.I2.P.I7. V.II. A.II.C. I9.

Fin. Juec.321. Cyprinus pinna ani officulorum II, cauda aeçuali.

Habitat in Europae fagnis, Lacrbus.

Caralfios repurgat; lïeri magnes. 
Cepha- 7. C. pinna ani radiis II, caulus. dae integra, corpore fubcylindtico.

Minf: Ad. Fr. p. 77. t. 30 .

Cyprinus pinna ani radiis

II, caude rotundata. D.II. P. I6.V. 9. A.II. C.I7. Art. gcr. 5. Syn. 7. Cyprinus oblongus macrolepidotus, pinna ani oniculis 11 .

D. .. P. .- V. .- A. II. C. --

Gron.mml: I. n.I2. idem. D. II. P. - V. 9. A. II. C.- mil: 2.p.3. idem.

Habitat in Europa meridionali.

* * Pinna caudae trifida.

auratus. 8. C. pinna ani gemina, caudae transuerfa trifurca. Fn. fitec. p. I25. t. 2.

Act. Stockb. 1740 p. 403 .

t. I. f. I - 8. idem D. $\frac{2}{18} \cdot$ P.r6.V.9.A.8,8. C.37. Gron.mif.I. n.I5. idem. D. 8. P. II. V.7.A. - - C. 20. mil:2. n.150. C. pin-

na ani fimplici, cauda trifurca.

Edw. all. t. 209 .

Pet. gaz. t. 78. $f \cdot 7$.

Habitat in Chinae, Iaponiae fiuniis.

Pifcis colitur in refis murrbinis ob aurean fulgorem,

tum multun pinnis varians. Radius fecundus ani pofice ferratus.

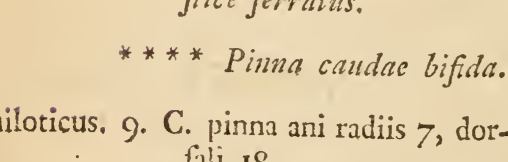

niloticus. 9. C. pinna ani radiis 7 , dor-

$* *^{*}$ Pinna caudac bifida.
niloticus, 9. C. pinna ani radiis 7 , dor-
fali 18.

Haffelqu. iter. 393. n. 94 .

D. I8. P. Ir. V.8.A.8. C. 44 .
Cyprinus rufefcens.
Habitat in Nilo.
D. $18 \cdot P \cdot \frac{I}{17} \cdot V \cdot 9 \cdot A \cdot 7 \cdot C \cdot 24$.

Phoxinus. 10. C. pinna ani radiis $8, \mathrm{ma-}$
cula fuica ad caudam,

Phoxinus. 10. C. pinna ani radiis $8, \mathrm{ma-}$
cula fuica ad caudam, corpore pellucido.

D.18. P.17.V.9.A. 7. C.19.

D. S. P.16. V. \&. A. 8. C.19.

Art. 




\section{PISCES ABDOMINALES. Cyprinus. 323}

Art. Syn. 12. Cyprinus tridactylus varius oblongus teretiufculus, pinna ani officulorum 8.

Habitat in Europa.

Aphya. II. C. pinna ani radiis 9, iridibus rubris, corpore pellucido. Fin. Juec. 331. D. 10.P.12. V.7.A.9. C.19.

It. Wrot. 232. Cyprinus minimus.

Avt. gen. 4. Jyn. I3. Spec.

D.II. P. 8.V.S. A. 9. C. 19. 30. Cyprinus biuncialis, irıdibus rubris, pinna ani officulorum 9 .

D... P... V... A. 9. C...

Habitat in Europae riunlis.

Leuci- 12. C. pinna ani radiis 10, fcus. dorfali 9 . Art. Syn. 9. Cyprinus noD. 9. P... V... A. I0. C. I9. uem digitorum, rutilo longior et anguftior, pinna ani radiorum decem. Habitat in Europa media.

Dobula. 13. C. pinna ani dorfalique radiis 10.

Art.Jyn. 10. Cyprinus peda-

D.10. P.15 V.9.A. 10.C. 19 . lis gracilis oblongus cras. fiulculus, dorfo craffo, pinna ani officulorum 9. Ilabitat in Europa media.

Grislagi- 14. C. pinna ani radiis II, pinne.

nis allentibus In.fuec. 325 . Art. gen. 5. Cyprinus obInngus, iride argentea, pinnis albentibus.

Gron. mul: 2. "1. IfS. Сyprinus iciem.

Art. Jyn. 5. Spec. 12. Cyprinus oblongus, figura rutili, pinna ani officulorum 10

Habitat in Europae lacubus.

D.II.P.II. V. 9. A. II. C.-$x_{4}$ 


\section{PISCES ABDOMINALES. Cyprinus.}

Idbarus. 15. C. pinna ani radiis 12, pinnis ventralibus fanguineis.

D. ro. P... V... A.r2.C. 19.

Habitat in Sueciae lacubus.

Rutilus, 16. C. pinna ani radiis 12 rubicunda. Fin. fuec. 329. Art. gen. 3. Jyn. 10. Jpec. 10. Cyprinus iride pinnis ventris ac ani plerumque rubentibus. $\quad$ D.13.P.15.V.9. A.12. C.17. Gron. muf. 1. n. 8. idem. D. 12. P. -. V. 9. A. I3. C. .ACt. $\mid \overline{p l}:$ 1741. $p \cdot 74 \cdot n$. 5 r. idem.

Act. VP. I74I. 21. 52. Rex

D.10.P.II. V. IO. A.15. C..van Ruy.

Habitat in Europae lacubus.

D. I0.P.12. V.9.A.15. C. 22.

Idus. 17. C. pinna ani radiis 13 rubra. Fin. fiec. 320. D.10.P. 18.V.10.A.13.C.19. Art. gen. 5. Jyn. I4. Jpec. 6. Cyprinus iride fublutea, pinnis ventralibus anique rubris.

D.Ir. P.-- V. IO. A. I3. C. I9, Gron. mil. I. p. 3. n. I3. idem.

Habitat in Europae aquis dulcibus.

Orfus. 18. C. pinna ani radiis I3. Art. Jyn. 6. Cyprinus Orfus dikus.

Rai. pifc. IIg. Rutilus latior f. Rubellio fluuiatilis.

Meyer. thier. 2. t.94. Orf.

D. 10.P.19. V.9.A. I3. C... Habitat in Rheno, Angliae fluniis, lacubus.

Ery- 19. C. pinna ani radiis I4, throph- pinnis rubris. Fn. fuec. thalmus. $\quad 324$.

Art. gen 3. Jyn. 4. Spec.

9. Cyprinus iride pinnis

omnibus caudaque rubris. D. II.P.I6. V.10.A.I4. C. I9. Inbitat in Europa feptentrionali.

20. C. 


Yeses.

Iefes. 20. C. pinna ani radiis I4, roftro rotundato.

D. - P. .- V. .- A. -. C..-

Art. Jyn. 7. Cyprinus cubitalis, pinna ani officulorum I4.

Habitat in Germania.

Nafus. 2I. C. pinna ani radiis 14 , roftro prominente. Art. gen. 5. Jyn. 6.

Gron. mut: 2. I47. idem. D.II. P.7.V.9.A. I2. C. 25.

Habitat in Rheno flunio.

Afpius. 22. C. pinna ani radiis 16 , maxilla inferiore longiore insurua. Fn. fisec. 319. Art. gen. 6. Jyn. 14. Jpec. 14. Cyprinus maxilla inferiore longiore cum apice eleuato, pinna ani officulorum 15 .

Habitat in Sueciae lacubus.

D.II.P.IS.V.IO. A. I6.C. 19.

Albur- 23. C. pinna ani radiis 20. nus. Fin. Jitec. 330.

Art. gen. 6. Jyn. 10. Jpec.

17. Cyprinus quincuncialis, pinna ani officulorum 20.

D. 10. P.14.V.9. A. 21. C. -. Gron. muf: I. 13. I0. idem. D. 9. P.-.V. 9. A. 22. C. .ACr. I'pl: 1741. p. 75. $n$.

58. Koning van afterling. D. s. P.I4. V. 8. A. I8. C. 20. Habitat in Luropae aquis dulcibus.

Vimba. 24. C. pinna ani radiis 24, roftro naliformi. Fin. jul. 326. Art. gen. 6. Jyw. I4. Jpec. 18. Cyprinus roftro naliformi, dorfo acuminato, pinna ani officulorum 24. D.ir. P.IG.V.ro. A.24.C. Ig. Habitat in Sueciae lacubus.

Dentex. 25. C. pinna ani radiis 26 , ore dentibus indique moluribus.

$$
\mathrm{X}_{5} \text { D.10.P.14.V.9.A.26.C.I9.: }
$$




\section{PISCES ABDOMINALES. Cyprinus.}

Hafjelqu. itin. 395. Salmo dentex.

Habitat in Nilo.

D.10.P.15.V.10. A.24.C.25.

Ob dentes fingularis. Sparis fimilis, fed facies et membr. branchioftega omnino Cyprini; pinnam adifofam non vidi.

Brama. 26. C. pinna ani radiis 27. pinnis fufcis. Fr. Jivec. 318 . Avt.gen. 6. $\int y n$. 4. $\int \mathrm{pec} .22$. Cyprinus pinnis omnibus nigrefcentibus, pinna ani officulorum 27.

D.I2.P.I7.V.9.A.27.C.19. Gron. mul: 1. n. 14. idem. D.11. P.--V.10. A.27.C.Habitat in Europae lacubus.

cultratus. 27. C. pinna ani radiis 30 , linea laterali declinata, ventre acutisfimo. It. ficin. 82. $t .2$.

Habitat in M. Baltico.

Bjoerkna

Bioer- 28. C. pinna ani radiis 35 . lina. Fn. Jinec. 328. Art. gen. 3. Jju. 13. Jpec. D. II.P.15. V. 9.A.35.C.19. 20. Cyprinus quincuncialis, pinna ani osficulorum 25. D.Ir. P.15. V.9.A.25.C.19. Habitat in Sueciae lacubus.

Farenus. 29. C. pinna ani radiis 37 , iride flaua. Art. Jpec. 23. Fn. $\int u .327 . \quad$ D.II. P. I8. V. I0.A.37. C. I9. Habitat in Sueciae Lacu Maclero.

Ballerus. 30. C. pinna ani radiis 40. Fn. Jike. 323 .

Art. gen. 3. Jyn. 12. Spec.

23. Cyprinus admodum

latus et tenuis, pinna ani oficulorum $40 . \quad$ D.II.P.I6.V.IO.A.40.C.I9. Habitat in Europac lacubus. 




\section{BR ANCHIOSTEGI.}

162. MORMYRVS. Caput: Dentes plures, emarginati.

Apcritur linearis, pone capitis latera.

Corpus fquamis imbricatis.

Pimate ventrales funt abdo. minales.

cyprinoi- I. M. cauda bifida acuta.

des. Mitf. Ad. Fi. 2. p. . D.27.P. 9. V.6.A.32. C.19. Habitat in Nilo.

anguilloi-2. M. cauda bifida obtufa.
des.
Mul. Ad. Fr.2. p. .
Hasclqu. iter. 398. Mor-
D.26.P. I0. V.6. A. 4I. C. I9. myrus Cafchive.
Habitat in Nilo.

163. BALISTES. Caput compreffum. Dintes incifores vtrinque 8 paralleli. Apertura fupra pinnas pérorales. Corpus compreffum fquamis corio coadunatis. Aldomen carinatum.

Monoce- I. B. pinna capitis vniradiata.

ros.

MI!! Ad. I\%.2. p. . D D.I,46.P.I4.V.o.A.50.C.I2. Osb. itur. no. Baliftes Mo- noceros.
D.I, 47.P.I3. V.o.A. j̄I. C.I2.

Catcsb. sar. 2.t. 19. Vnicornu pilcis bahamenlis.

Habi-

Apertura Branchiarwm in boc ordine of rima, absque meubrana enidesti. 
Habitat in Afia, America.

Carina abdominis intra cuterm.

tomen- 2. B. pinna capitis biradiata, tofus. corpore pofterius fubuillofo.

Gron. muf. I. "1. II4. Baliftes aculeis dorfi 2, lateribus verfus caudam hirfutis.

Cluf: exot. 143. Rai. pifc. D. $\frac{1}{2}, 29$. P.10. V.0.A.27.C. 47. Monoceros pifcis. Marcgr. bra/. 154. Pira-aca. Habitat in America.

papillo- 3. B. pinna dorfali anteriore fus. biradiata, corpore papillofo. Muf. Ad. Fr. 2. P... D. $\frac{x}{2}, 29$. P.13. V.0. A. 21. C.12. Habitat. .

vertuco- 4 . B. pinna dorfali anteriore

fus. triradiata, cauda ordine triplici verrucarum. $M u y$ : Ad. Fr. I. p. 57. t. 27. f. 4 .

Habitat in India.

D.3,24.P.13. V.o. A.21. C.12.

aculea- 5. B. pinna dorfali anteriore

tus. triradiata, caudae lateribus fpinis recumbentibus.

It. Wgoth. I38. Oftracion comprefius, fafciis nigris, cauda lateribus muricata.

D. 3, 24.P.13. V.o. A.21.C.I2.

Habitat in India.

Simillimus priori ftatura et radiis; differt colore, fed imprimis nota illa fingulari, quod ad latera caudae vtrinque notetur quadruplici circiter ordine fpinarum recumbentium; cum praecedens eodem loco verrucas rotundas fäbras gerat.

6. B. 


PIS CES BRANCHIOS TEGI. Baliftes.

Vetula. 6. B. pinna dorfali anteriore triradiata, ventrali longitudinali, caudali bifida.

Catesb. car. 2. p. 22. t.

D.3,30.P.16.V.I6. A.28.C.12. 22. Turdus oculo radiato.

Art. gen. 53. Syn. 8. Baliftes aculeis dorfi tribus, cauda bifurca.

Osbeck. iter. 294. Baliftes Vetula.

Broron. inm. 456. Baliftes

D.3,30.1'.15.V.I7.A.27. C.I2. maior fafciata, dorfo triacantho, cauda bifisrca: radiis exterioribus longiffimis.

Habitut ad infulam Afcenfionis.

ringens. 7 . B. pinna dorfali anteriore triradiata, lateribus capitis triplicatis, cauda bifida. Mul: Ad. Fir. I. p. 58. D.3,29. P.15. V.o. A.26. C. 12. It. Wgoth. 139. Oftracion compreffus rufefcens, pinnae dorfalis radio longo retrorfum dentato. D.3,30.P.10.V.o. A.26. C.IO. Osbeck. iter. 295. Baliftes nigra. D.2,34.P.16.V.o.A.31. C.I3. Habitat ad inf. Afcenfionis.

D.3,30.P.14.V.I2. A.28.C.12。 


\section{$33^{\circ}$ PISCES BRANCHIOSTEGI. Oftracion.}

\section{OSTRACION. Caput: Dentes vtrinque ro por- recti, teretes obtufufculi. Apertura.. \\ Corpus offe integro obtectum. Pinata ventrales nullae.}

triqueter. I. O. trigonus muticus. Muff: Ad. Fi. x. p. 6o. O. ftracion polyodon inermis triqueter. $\quad$ D.IO.P.I2.V.O.A. I0. C. IO. Art. gen. 57. $\int y n .85 . \quad 0$. ftracion triangulus, tuberculis exiguis innumeris, aculeis carens. D.10.P.12.V.o.A.10.C. I0. Habitat in India.

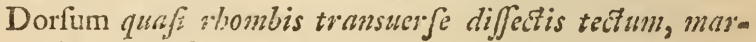
gine repandun.

trigonus. 2. O. trigonus fpinis fubcaudalibus 2 , pinna dorfali radiis XIV.

Art. gen. 56. $\int y n .85$. O. ftracion triangulus, limbis figurarum hexagonarum eminentibus, aculeis 2 in imo ventre.

It. fcan. I6o. Oftracion polyodon tetragonus, abdomine pone bicorni. D.I4. P.IO.V. 0.A. 9. C.7. Habitat in Iridia.

bicauda- 3. O. trigonus, f pinis fublis. caudalibus 2 , pinna dorfali radiis decem. Art. gen. 57. Jyn. 85. Oftracion triangulatus, tuberculis hexazonis radiatis, aculeis 2 in imo ventre. D. IO.P.I2.V. O.A. IO. C.IO.

B. Art. gen. 57. Jjn. 85. Oftracion triangulatus totus maculofus ac tubere 


PISCES BRANCHIOSTEGI. Oftracion. 33 I

culofus, aculeis 2 in imo

ventre. D.IO.P.II. V.O.A.IO.C.IO.

Habitut in India.

tricornis. 4. O.trigonus, fpinis frontalibus duabus, dorfali vnica. Art. gen. 56. Jyn. 85. Oftracion triangulatus, aculcis 2 in capite er vnico longiore fuperne ad caud่am.

Habitut in India.

quadri- 5. O. trigonus, fpinis froncornis. talibus fubcardalibusque binis.

Art. gen. 56. Jyn. 85. Ontracion trianigulatus, 2 aculeis in fronte et totidem in imo ventre.

D.IO. P.Ir.V.O.A.IO.C.IO.

Habitat in Inùia.

cornu- 6. O. tetragonus, fpinis frontus. talibus fubcaudalibusque binis.

Mul: At. Fr. I. p. 59. Oftracion poíyodon terragonus, antice poltice dorloque fpinolus. $\quad$ D.9. P.10. V.0.A.9. C. 10.

Gron. muj: I. n. IIS. Oftracion quadrancularis, aculeis 2 in fronte et totidem in imo ventre. D. II. P. 9. V. 0. A. -. C. 5 . Will. iclot. t. 7. 13. $f$. 1 . Pifcis cornucus. Eunt. ias. 79 .

Habitat in India.

cubercu- 7. O. tetragonus muticus, tulatus. berculis dorfalibus 4 . Art. gcm.55. linn. 84. Oltracion quadrangulus, tuherculis + mioribus in dorfo.

Ilabitat ili India. 


\section{PISCES BRANCHIOSTEGI. Oftracion.}

gibbofus. 8. O. tetragonus muticus gibbofus.

Art. gen. 55. Jyn. 83. Oftracion quadrangulus gibbofus.

Habitat in India.

cubicus. 9. O. tetragonus muticus, 12teribus planiufculis. Mus. Ad. Fr. I. p. 59. Oftracion polyodon tetragonus inermis.

D. 9. P. I0. V.o.A.10. C. I0.

It. Wgoth. p. 138. nomen Artedii.

Avt. gen.56. Jyn. 85. Oftracion quadrangulus maculis variis plurimis. Habitat in India.

D. Iо. P. 8. V.o. A. 9. C. Iо. D. 9. P. Iо. V. ०. A. 8. C. Iо.

165. TETRAODON. Caput: Maxillae offeae, porrectae, apice bipartitae. Apertura linearis.

Corpus fubtus ventricofum muricatum.

Pinzace ventrales nullae.

teftudi. I. T. abdomine plano laeneus. uiore, dorio futuris curuis albis pißzo.

Art. gen. 60. Jyn. 86. Oftracion oblongus glaber, corpore figuris variis ornato.

D... P. I4. V.0. A. 6. C. 9.

Amoen. acad. I. p. 309. t.

I4. $f .3$ idem.

D.6. P. I4. V. o. A.6. C.9. Habitat in India.

Corpus fupra feabriufculum, fubtus punctis pertufinn, intra quae aculei latent.

lagoce- 2. T.abdomine aculeato, cor-

phalus. a pore heui, humeris prominentibus. 
Setrodon, wide p.243.

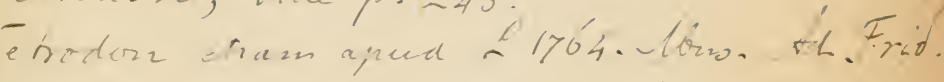





\section{PISCES BRANCHIOSTEGI. Tetraodon。 333}

Niuf. $1 d$. Fr. I. p. 59. Oftracion tetraodon ventricofus, abcionine muricato.

Art. ger. 53. fyn. 86. O-

D.10. P.18. V.0. A.8. C.10. fitracion catheioplateo-ob. longus, ventre tantum aculeato et linbrotundo.

Anown. acad. I. p. 310. $t$. If. f. 4. idem. D. 9. P. 17. V. 0. A. 9. C.7. Gron.mif. I. n. 126. O. ftracion cathernplateus tetraodon crimpreflus maculolus feaber. Habitat in India.

lineatus. 3. T. fafciis longitudinalibus fulcis p.llidisque. Aluf:

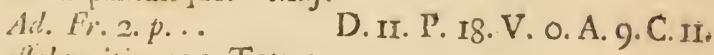
Hulfilqu. itin.400. Tetraodon Fuhuca. Habitat in Nilo.

D.I2. P. 2I. V.O. A. 9.C.II, Aculei ublominis venenati pungunt, quafi vrticatione.

ocella- 4. T. fafcia humerali ocellazus.

ta. Mul: Ad. F\%.2. p.... D. I4. P.IS. V.O.A.12. C. \% Osbeck. itin. 226. Diodon ocellatus. D. I5. P.IS. V.O. A.II.C.S. It. $\int \dot{c} a n .260$. Oftracion maculotis, abdomine muricato.

Art.gen.5S.jyn.85. OftraD. 12. P.20.V.O. A. 12. C. . . cion macululus, aculeis vndique denlis exiguis.

Hobitat in Aliae ot Aegypti aquis dulcibuss. l'enematus pigcis, ati praticidens.

hifpidus. 5. T. totus hifpidus papillis fetaceis.

Clirs. Lagerfls: 23. (Ontracion tetradon ventricos fis, corpore to:o muricato. D.9. P.17. V.O.A.10.C. 10. 
Art. gen. 58. jyn. 83. Oftracion tetracion iphaericus, aculeis vndique $\mathrm{e}$. xiguis.

Halitut in India.

Mola. 6. T. heuis compreffus, cauda truncata : pinina breuiflima dorfali analique annexa.

Art.gen. 6r. $\int y n$. 83. Ofrracion cathetoplateus fubrotundus inermis afper, pinnis petoralibus horizontalibus, foraminibus 4 in capite. 1). - P. 14 . V... A. .. C... Ait.bonon. 2.pp.2.p.297. t. 17. Mola.

B. Gron. my: 1. n. 125. Oftracion cathetoplateus fubcomprefus, pinna dorfi et ani cum cauda continuatis.

Habitat in M. Mediterraneo.

166. DIOJ)ON. Caput: Maxillae offeae, porrectae, indiuifae.

Apertura linearis.

Corpus fpinis acutis mobilibus vndique adfperfum.

Pinnae ventrales nullae.

Atringa. I. D. fphaericus, aculeis triquetris.

Art. gen. 59. Syn. 86. O. ftracion bidens fphaericus, aculeis vndique denlis triquerris.

Habitat in India.

rețicula- 2. D. fubrotundus, aculeis tri-

tus. quetris. 


Art.gen. 59. Jyn. 86. O. ftracion lubrotundus, iculeis vndique breuibus triquetris raris.

Habitat in India.

echina- 3. D. fubrotundus, aculeis tus. bali triquetris. Art.gen. 6o. Jyn. 5. O. ftracion fubrotun 'us, aculeis vndique $\mathrm{d} \mathrm{f}^{-}$- $\mathrm{s} \mathrm{ba}$ fi triquetris.

Habitat in India.

fpinofus. 4. D. fubrotundus, aruleis planis, abdomine laeui. Art. gen. 59. Jyn. 86. Ofrracion fubrotundus, aculeis vndique breuibus planis, ventre glabro.

Habitat in India.

Hyfrix. 5. D. oblongus, aculeis teretibus.

Mirj: Ad. Fr. r. p. 58. 0 ftracion diodon, corpore fpinis vndique armato. D. 14. P.22. V.0.A.14.C.9. Avt. gen. 60. Jyn. s6. O. ftracion conico - oblongus, aculeis vndique longis teretiformibus imprimis in lateribus.

Amoen. acad. I. p. 310. idem.

It. $\int c a n z .285$. idem.

Habitat in India.

D. 14. P.22. V. O. A. I4. C. 9.

holocan- 6. D. aculeis capitis collique thus. Iongioribus. Art. gen. 60. Syn. 86. O. ftracion oblongus holocanthus, aculeis longiffimisteretiformibus in capite imprimis et in collo. Habitat in India. 


\section{PISCES BRANCHIOSTEGI. Diodon.}

raninus. 7. D. pima dorf longifrima. Avt. gen. 59. Jin. 86. O. ftracion rorundo - oblongus, tuberculis vtrinque, pinna dorí longifima.

Habitat in India.

167. CENTRISCVS. Caput productum in Roftrum angurtifimum. Apertura repanda.

- Corpizs dorfo loricatum Ofre poftice mucronato recto longitudine corporis. Abdonien carinatum.

Pinna ventralis vnica.

fcutatus. I. CENTRISCVST. Gron.muf.

2. p. J.7I. t. 7.f. 3. D. 3, 9. P. I0.V.6. A. Ir. C.9. Valcutyn. amb. 4. p. 420 .

n. $243 \cdot f .243,254$. I-

kan Pifau.

Habitat in India oricntali.

Singularis pilcis lovicatus tefla offea longitudinali poftice in Jinamin terninata, fib qua casda; fid, inter Jpinann et caudain, pinnae dorfi fint.

\section{Syngmathses.}

168. SYNGNATVS. Caput: Roftrum fubcylindricum, ore operculato maxilla inferiore.

Apertura branchiarum operculis claufis; nucha foramine fpirans.

Corpus articulatum. Pinzace veratrales nullae,

Typhle. I. S. pinnis caudae ani pectoralibusque radiatis, corpore fexangulato. 


Ant. gin. I. Sin. I. Spec.

3. Synsnarhus corpore

rimedio hexigono, cauda

pinnats. in. Juec. 336. D.36. P.I4.V.O.A.3. C. ro.

Govn.mef. I. 72. 4. iden. 1.7. P. 9. V.0. A.0. C.I2. Ilabitat in Europa.

Laninac corporis trunci I8, cauda: 36.

Acus. 2. S. pinnis caudae ani pectorilibusgue radiatis, corpore feptem-angulato.

Art. ge's. I. Jin. 2. Jpic. 2.

syneriathus corpure me-

div heprageno, caudla pin-

11.12. Fin. jute.335. D.38. P.r2. V. O. A. 5. C.ro.

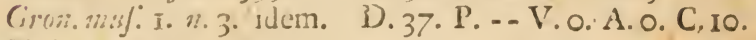

ll.bibitat in Luropa.

Lasnuarae curporis trunci 20 , caudae 43.

pelagi- 3. S. pinnis peftcralibus cau-

cus. dieque radiatis, ani nulla, cor ore fepremangulato.

Osbeck. itin. 305. Syngnathui pelagicus. $\quad$ D. 3I.P. .4. V.O. A. O. C. IO.

IJubitat in liven natante.

Lamisue cusporis beptagoni IS, Candae tetragonae 32.

acquo- 4. S. pinna caudae radiata,

reus. pectoraibus anique nullis, corpore angुulato.

D. 3०. P.०. V.o. A. ०. C. 5 . Habitat in Pclago.

Ophi- 5. S. pinnis caudae ani pectodion.

ralibu gue nullis, corpote tereri.

Ait. gin. I. Jjn. 2 Spec. I. Syronsthus teres, pinnis peetoralibus cudaeçue carens. lin. fivec. 334 . D.34. P.O. V.O. A. O. C. o. Ciron. m\% . n. 2. idem. D. 42. P.O. V.O. A. O. C. O. Hablibut in Europa.

harbarus. 6. S. pinnis caudae anique nul-

lis, corpore fexamgulato. D.43. P.22.V.0. A. O. C. o. Ilábicat in P'slago. 


\section{PISCES BRANCHIOS TEGI. Syngnathus.}

Hippo- 7. S. pinra caudae nulla, corcampus. pore feptemangulato tuberculato, cauda quadrangulata. D.20.P.18. V. O. A.4. C. O.

Art. gen. I. Jyn. I. Syngnathus corpore quadrangulo, pinna caudae carens. D.35. P... V. O. A. o. C. o. Olear.mul.53. t. 26. f. 4. Hippocampus.

Bradl. natur. t. 4. f. 3 . Equus marinus. Habitat in Pelago. Laminae corporis trunci 17 , caudae 45 .

169. PEGASVS. Caput: Os probofcide retractili; maxilla fuperiore denticulata, fub Roftro enfiformi, lineari, vtrinque ferrato.

Apertura branchiarum ante pinnas peetorales.

Corpus articulatum offeis incifuris, cataphractum. Pinnae ventrales abdominales.

volitans. I. pEgASvs. Muf. Adl. Fr.

2. p... D. 5. P. I0. V.2. A. 5. C.7. Gron. muf. I. $n .1+6 . \mathrm{Pi}$ ficiculus amboinentis volans offeo tuberculofus, proboficide ferrata.

Rusylich. the : t. 7. f. 2. D.

D. 4. P. 10. V. I. A. 5.C.:Habitut in Amboina.

Quis, nif vidiffer, pifces habitare fub vnda, crederet? 





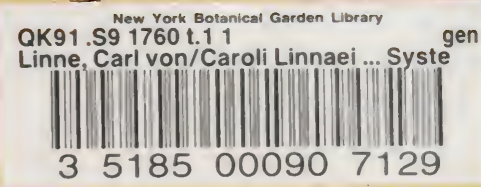

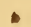




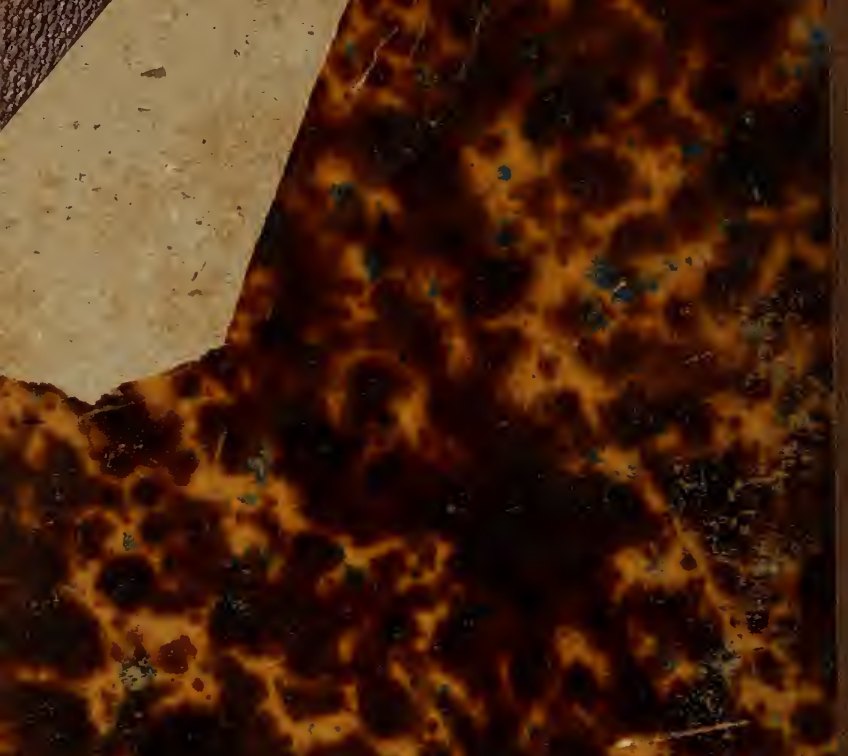

$51+5-5 x+5 x^{3}$

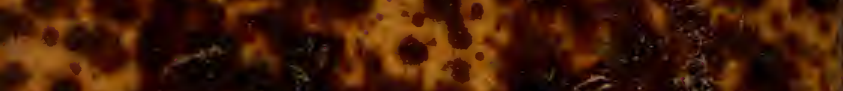

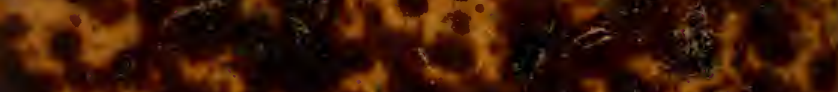
seis $\tan ^{2} x^{3}$

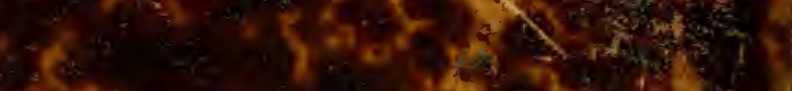

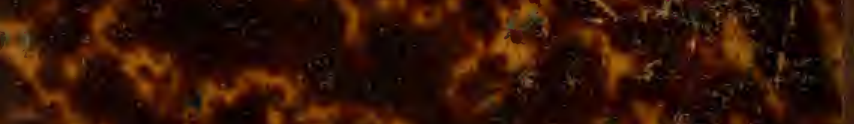
3) Mean $x_{5}:$

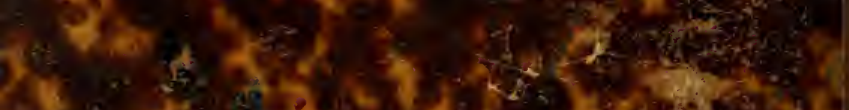

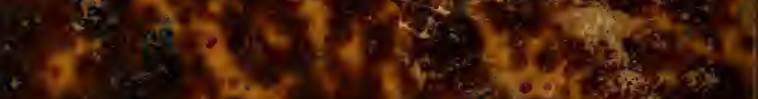
has pyors

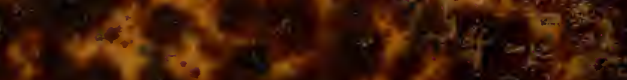
4. $500-2 x^{-1}$ 60 wo - " 1.

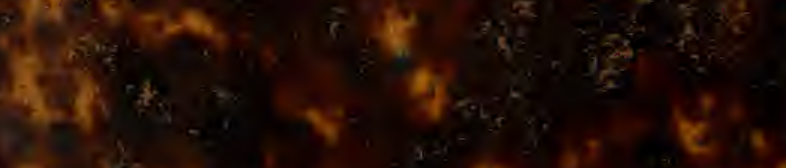
$4 x+y$, 4 है

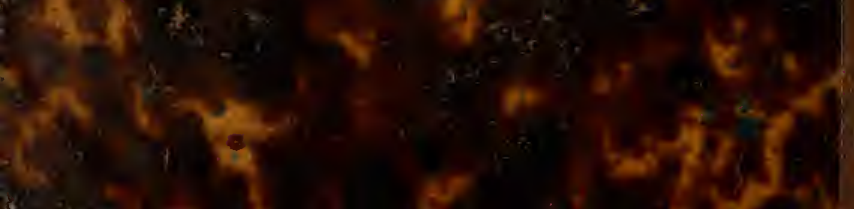

